Nova Southeastern University

Nova Southeastern University

\title{
Implications of a Multicomponent Sleep Program After Brain Injury: A Mixed Methods Study
}

\author{
Brandi M. Fulwider \\ Nova Southeastern University
}

Follow this and additional works at: https://nsuworks.nova.edu/hpd_ot_student_dissertations

Part of the Occupational Therapy Commons

\begin{abstract}
All rights reserved. This publication is intended for use solely by faculty, students, and staff of Nova Southeastern University. No part of this publication may be reproduced, distributed, or transmitted in any form or by any means, now known or later developed, including but not limited to photocopying, recording, or other electronic or mechanical methods, without the prior written permission of the author or the publisher.
\end{abstract}

\section{NSUWorks Citation}

Brandi M. Fulwider. 2020. Implications of a Multicomponent Sleep Program After Brain Injury: A Mixed Methods Study. Doctoral dissertation. Nova Southeastern University. Retrieved from NSUWorks, College of Health Care Sciences - Occupational Therapy Department. (78) https://nsuworks.nova.edu/hpd_ot_student_dissertations/78.

This Dissertation is brought to you by the Department of Occupational Therapy at NSUWorks. It has been accepted for inclusion in Occupational Therapy Program Student Theses, Dissertations and Capstones by an authorized administrator of NSUWorks. For more information, please contact nsuworks@nova.edu. 
Implications of a Multicomponent Sleep Program After Brain Injury: A Mixed Methods Study

by

Brandi M. Fulwider

Submitted in partial fulfillment of the requirements for the degree of

Doctor of Philosophy in Occupational Therapy

Nova Southeastern University

Dr. Pallavi Patel College of Health Care Sciences

Department of Occupational Therapy

November 2020 


\section{NOVA SOUTHEASTERN UNIVERSITY \\ HEALTH PROFESSIONS DIVISION}

DR. PALLAVI PATEL COLLEGE OF HEALTH CARE SCIENCES

DEPARTMENT OF OCCUPATIONAL THERAPY

FORT LAUDERDALE, FL 33328

This dissertation, written by Brandi M. Fulwider under direction of her Dissertation Committee, and approved by all of its members, has been presented and accepted in partial fulfillment of requirements for the degree of

DOCTOR OF PHILOSOPHY

DISSERTATION COMMITTEE

Wendy Stav, PhD, OTR/L, FAOTA

Date

Chairperson of Dissertation Committee

Sarah Blaylock, PhD, OTR/L

Date

Dissertation Committee Member

Tony Gentry, PhD, OTR/L, FAOTA

Date

\section{Dissertation Committee Member}




\section{NOVA SOUTHEASTERN UNIVERSITY \\ HEALTH PROFESSIONS DIVISION \\ DR. PALLAVI PATEL COLLEGE OF HEALTH CARE SCIENCES \\ FORT LAUDERDALE, FL 33328}

\section{Certification}

We hereby certify that this dissertation, submitted by Brandi M. Fulwider, conforms to acceptable standards and is fully adequate in scope and quality to fulfill the dissertation requirement for the Doctor of Philosophy degree.

\section{Approved:}

Rick Davenport, Ph.D., OTR/L

Date

Ph.D. in Occupational Therapy Program Director

Rebecca Estes, Ph.D., OTR/L, CAPS

Date

Chair of Department of Occupational Therapy

Peter Taylor, Ph.D.

Date

Executive Associate Dean for Academic \& External Affairs

Stanley Wilson, PT, Ed.D., CEAS

Date

Dean, Dr. Pallavi Patel College of Health Care Sciences 


\begin{abstract}
Decreased sleep can negatively affect mental and physical health and can significantly disrupt participation in daily occupations and leisure interests. Even though sleep is an area of occupation, there is limited evidence on the effectiveness of occupational therapy interventions for sleep. The purpose of this embedded mixed methods research study was to identify how disruptions in sleep affected daily occupational performance and to understand the effectiveness of occupational therapydirected non-pharmacological sleep interventions for three participants in a community re-entry program for brain injury. This study utilized a single-subject research design during a 4- to 5-week multicomponent sleep program using multiple sleep-related outcome measures and daily sleep diary and Fitbit data. Semi-structured interviews were also utilized. Identified qualitative themes were changes in sleep patterns and living with sleep disturbance. Participants reported decreased energy and fatigue after disrupted sleep but also reported still being able to complete daily occupations. Quantitative results yielded variable changes in sleep, with overall positive responses to interventions noted in a cumulative effect over time. The most significant improvements were in sleep efficiency and total sleep time for all three participants using sleep diary data. Additionally, two participants reported decreased sleepiness and increased functional outcomes after participating in the sleep interventions. In conclusion, simple behavioral, occupational, and environmental adaptations can improve sleep after brain injury. This study also further supports the role of occupational therapy in addressing sleep in education, practice, and research.
\end{abstract}




\section{Acknowledgments}

I am so grateful to the many people who helped and supported me though out this dissertation journey. I would like to thank my dissertation committee for their continued support and guidance. I am particularly grateful for my committee chair, Dr. Wendy Stav, who provided me with endless encouragement, insight, and wisdom from start to finish. Dr. Sarah Blaylock provided additional insight in regards to methodology decisions and data interpretation, and Dr. Tony Gentry added his expertise in regards to working with individuals with cognitive deficits and in addressing sleep in occupational therapy. I want to thank each of my committee members for their time and commitment in helping me to see this project through to the end. I could not have done it without each one of them.

I would also like to thank the occupational therapists in the REST program at Colorado State University, and I am extremely grateful for the opportunity to have attended the Cognitive Behavioral Therapy for Insomnia for Occupational Therapists training course at this institution. This training course, and the gracious time spent with the occupational therapists who run the program, gave me the foundation and the confidence that I needed to address sleep deficits and to complete this research study.

I also appreciate the assistance of the lead occupational therapist in the community reentry program, who helped me to refine study procedures and to recruit study participants. Finally, I would like to thank the participants and the family members who assisted the participants in my study to complete the sleep program. Without their participation, I would not have been able to complete the study and finish this dissertation journey. I am forever grateful to each of these individuals. 


\section{Table of Contents}

Chapter One: Introduction .................................................................................................. 1

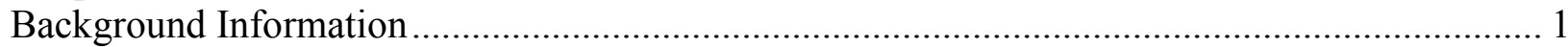

Statement of the Research Problem and Significance of Project.................................................... 2

Relevance to Occupational Therapy ……………………...................................................... 3

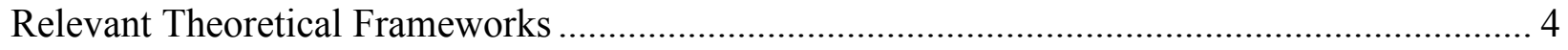

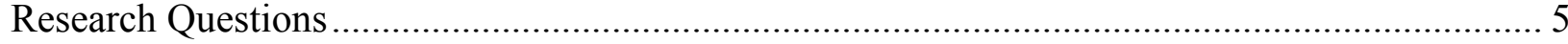

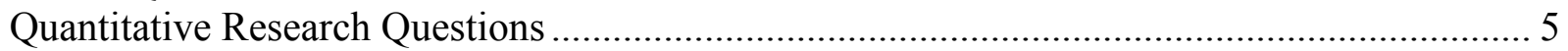

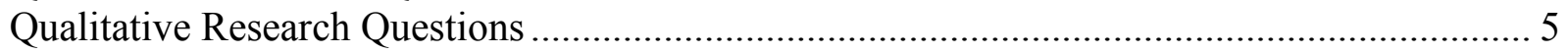

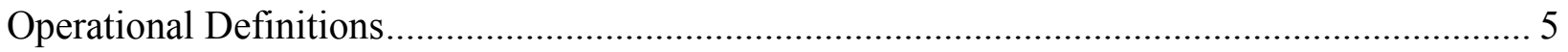

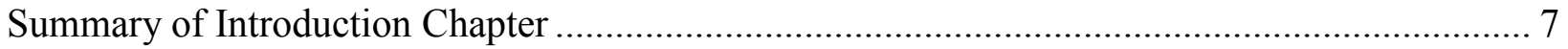

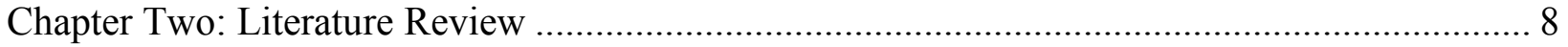

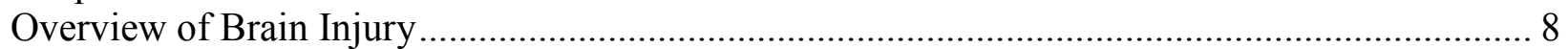

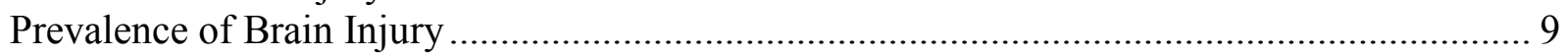

Functional Implications of Brain Injury ………………............................................ 10

Functional Recovery after Brain Injury ……………................................................. 10

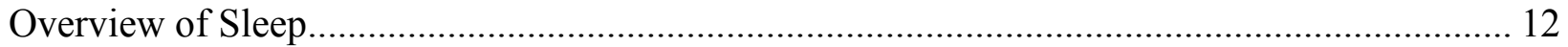

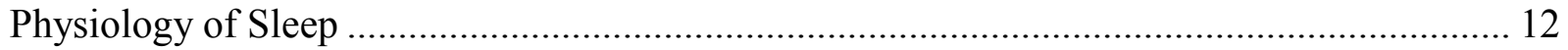

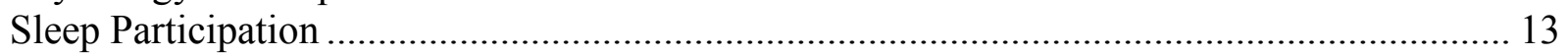

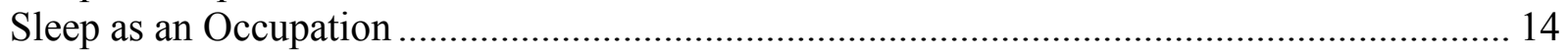

Healthy Sleep Habits................................................................................................ 15

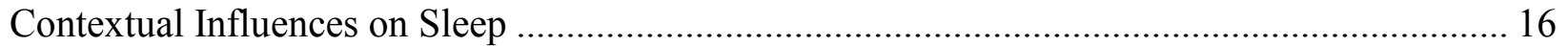

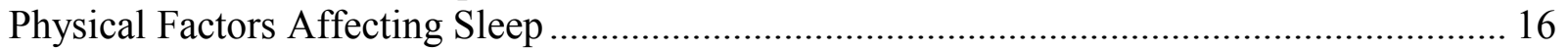

Socio-cultural Factors Affecting Sleep …………………….............................................. 18

Temporal Factors Affecting Sleep ……………………................................................... 18

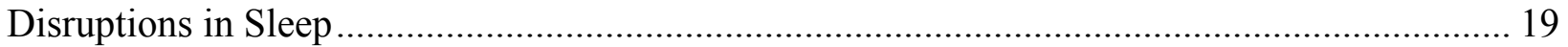

Functional Implications of Decreased Sleep.................................................................... 19

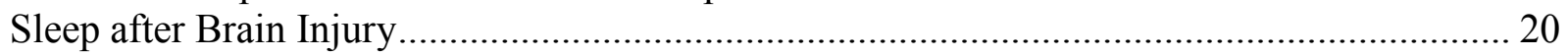

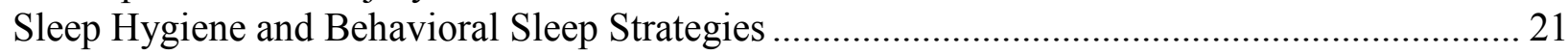

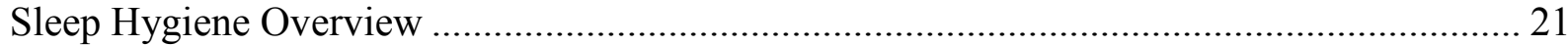

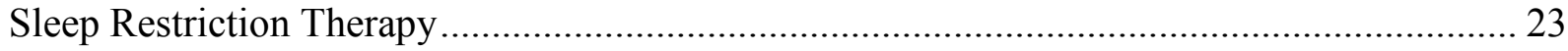

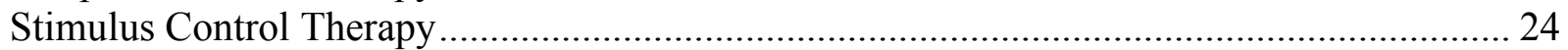

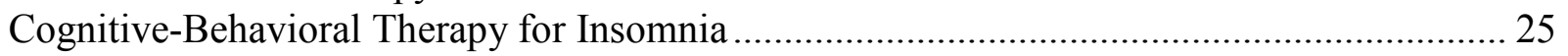

Effectiveness and Implications of Sleep Hygiene ………………...................................... 25

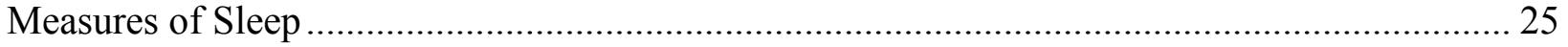

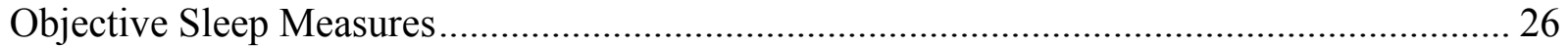

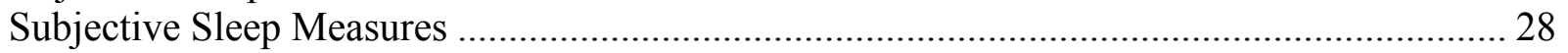

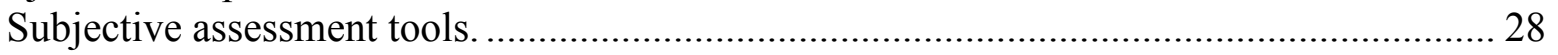

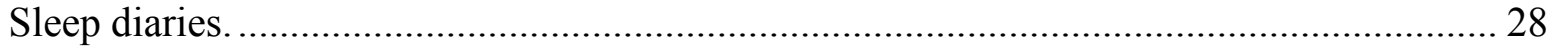

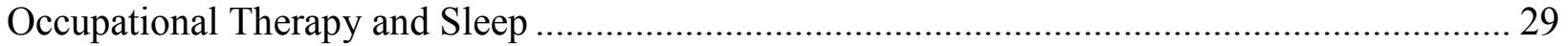

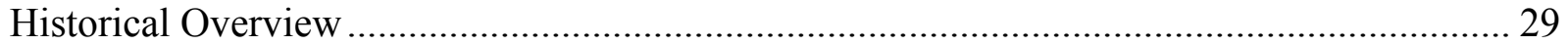

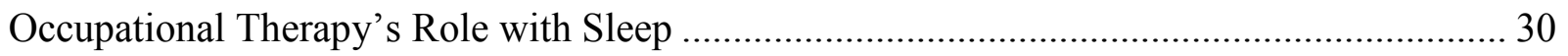

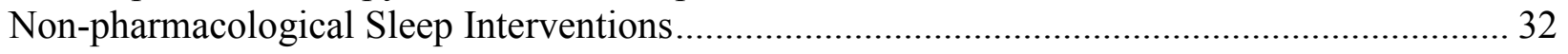

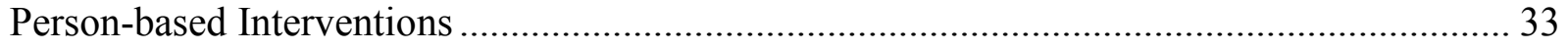

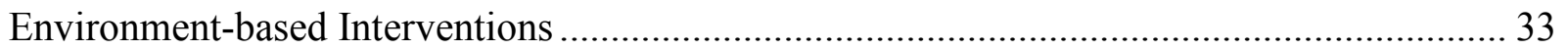

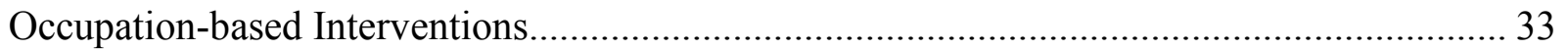




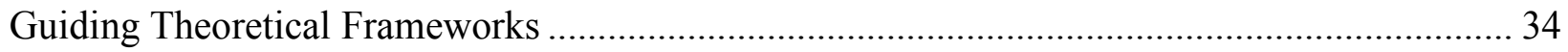

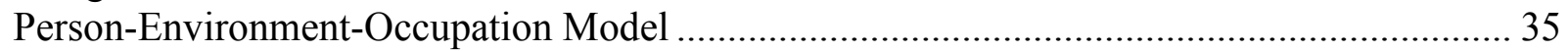

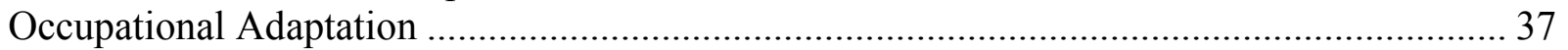

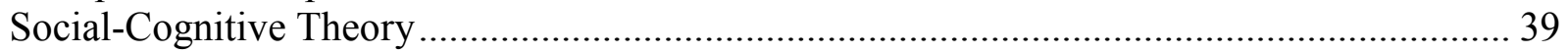

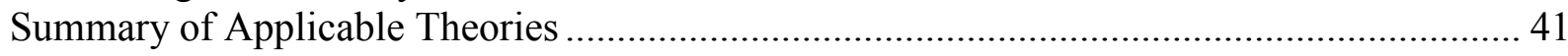

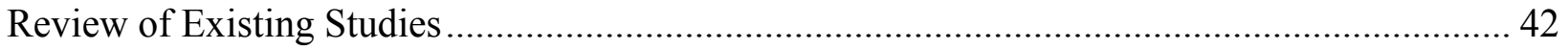

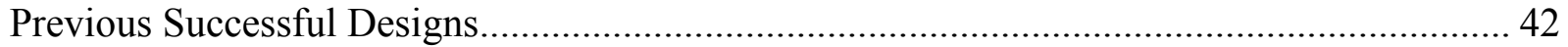

Mixed methods designs.......................................................................................... 42

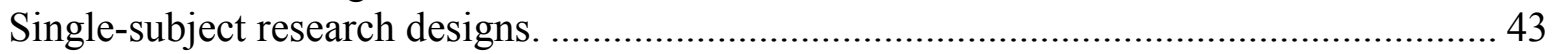

Designs and Methodologies Needing Improvement ................................................................ 43

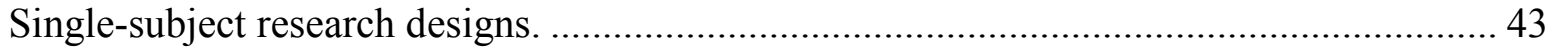

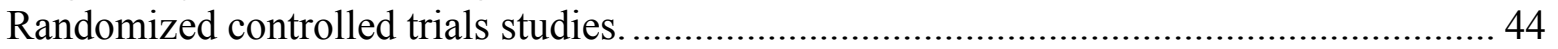

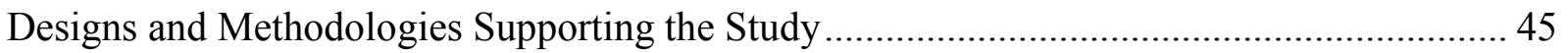

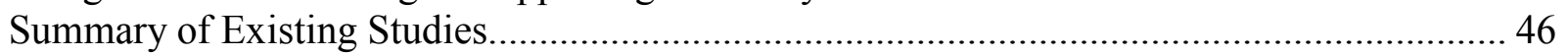

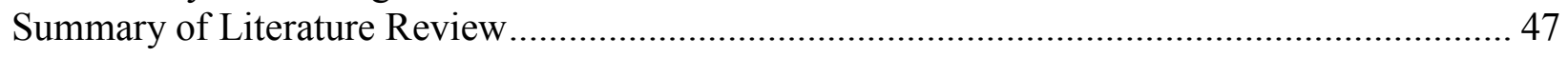

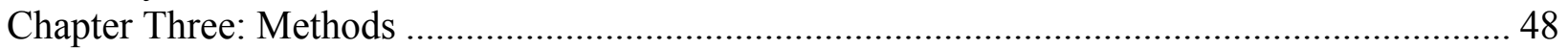

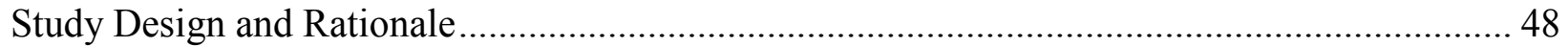

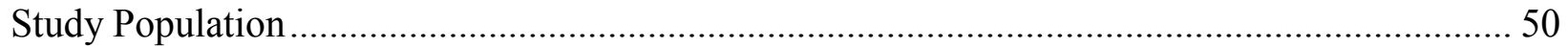

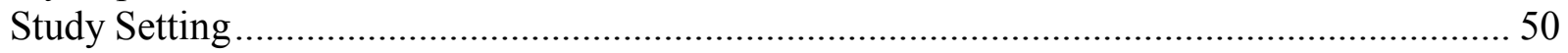

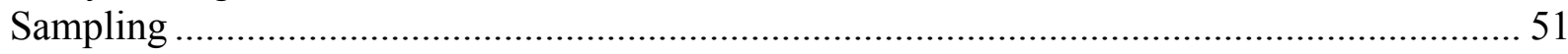

Inclusion and Exclusion Criteria................................................................................. 52

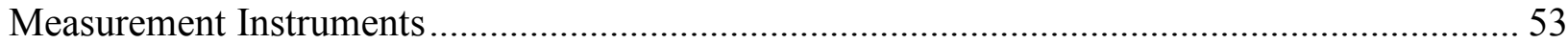

Pre-Screening Data Collection Instruments ................................................................... 54

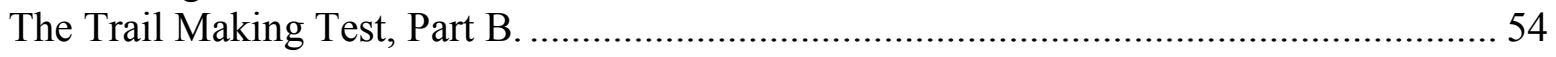

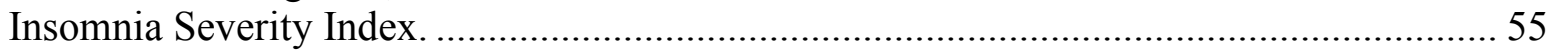

Outcome Measures Data Collection Instruments …………................................................. 56

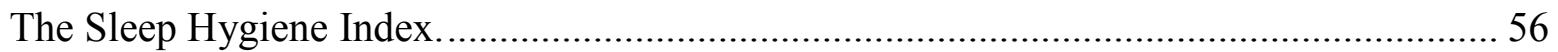

The Functional Outcomes of Sleep Questionnaire. ............................................................... 57

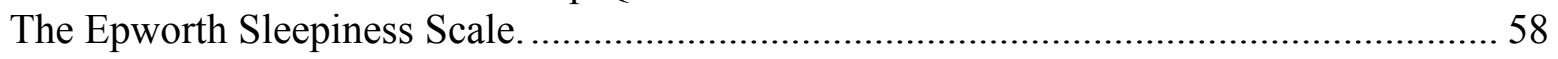

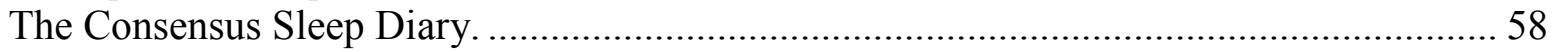

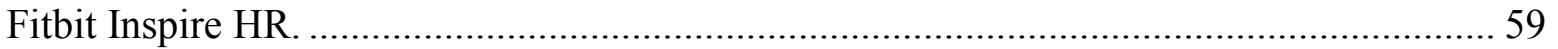

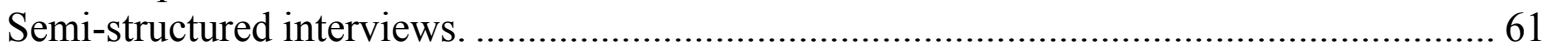

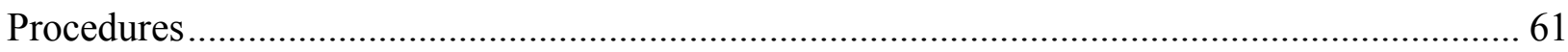

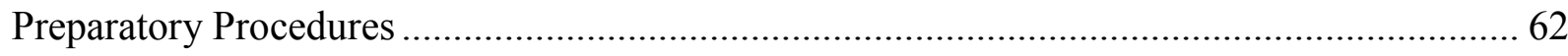

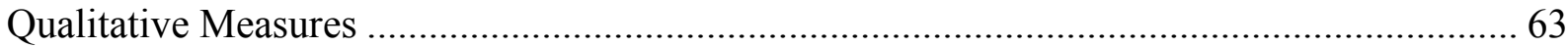

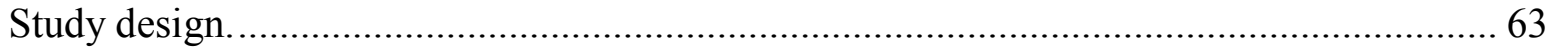

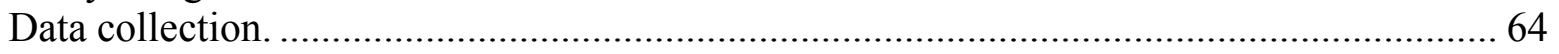

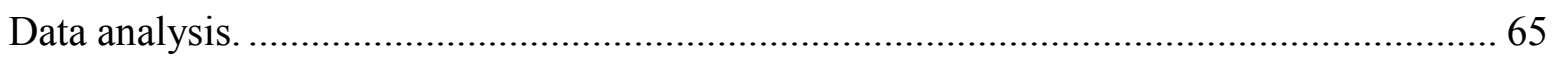

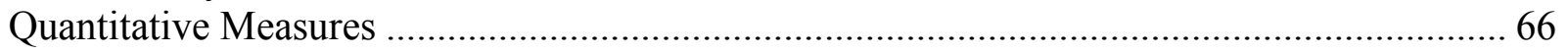

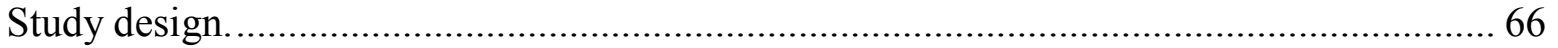

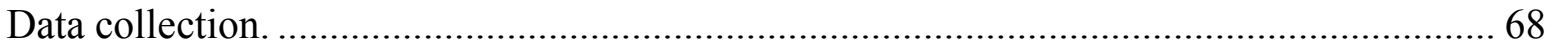

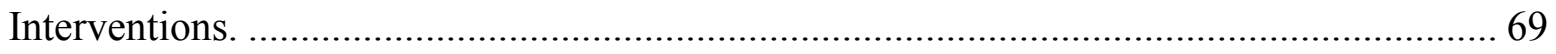

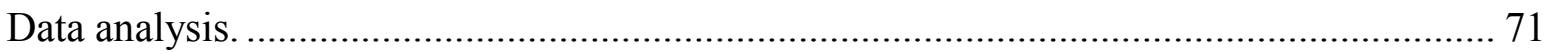

Integration of Quantitative and Qualitative Data ................................................................. 73

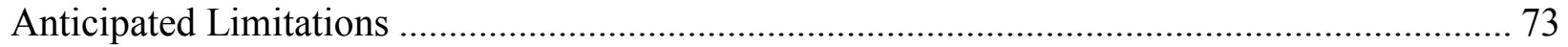

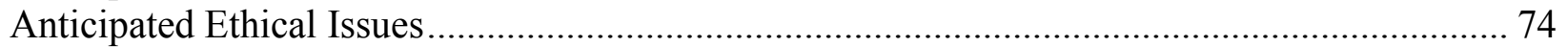




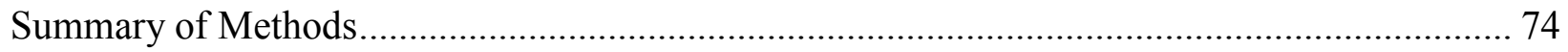

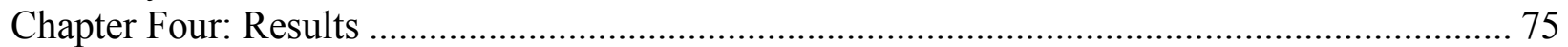

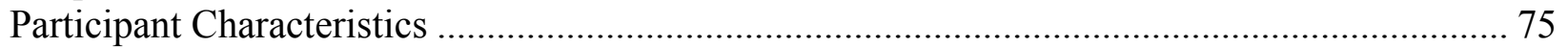

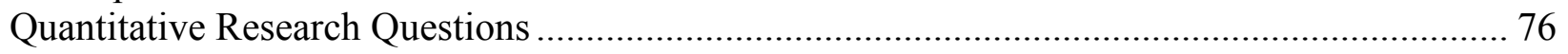

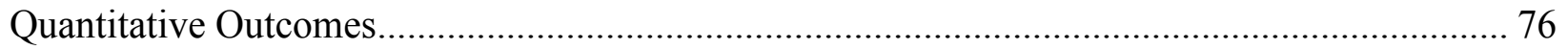

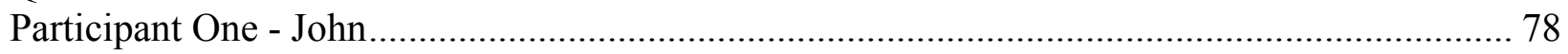

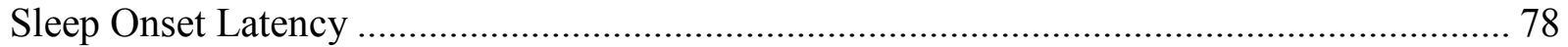

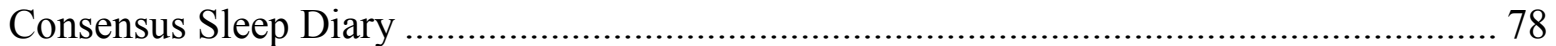

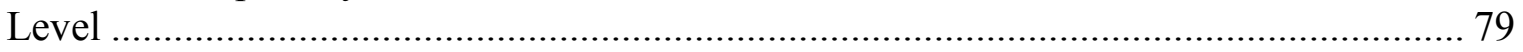

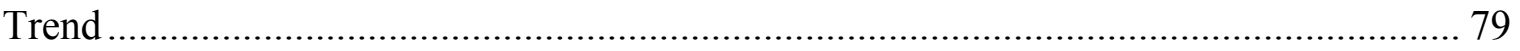

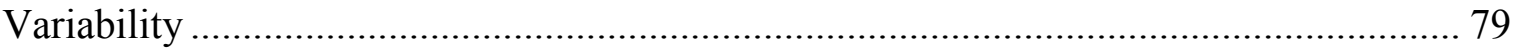

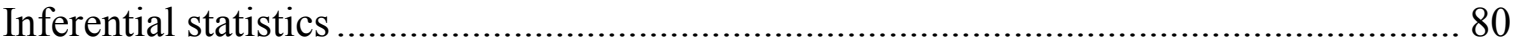

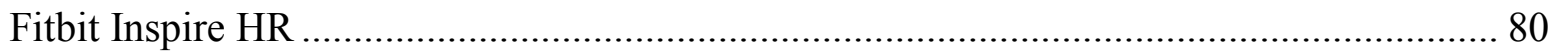

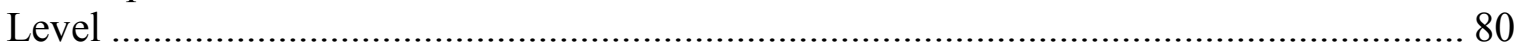

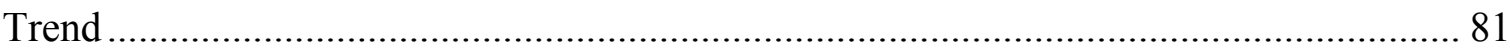

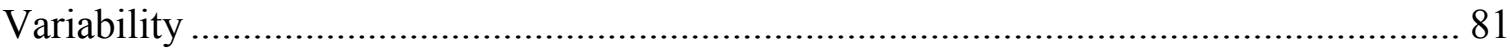

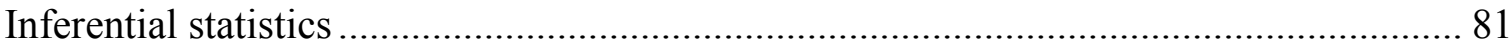

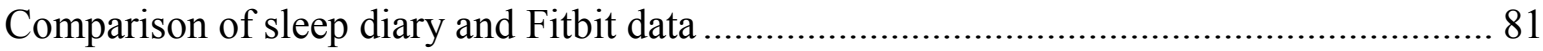

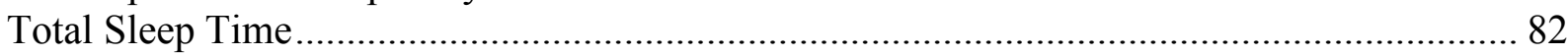

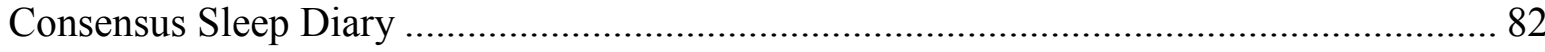

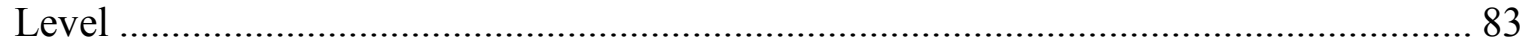

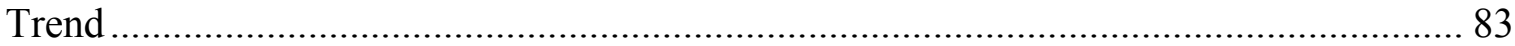

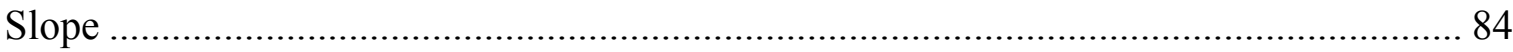

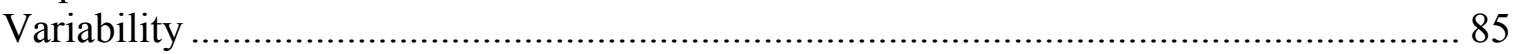

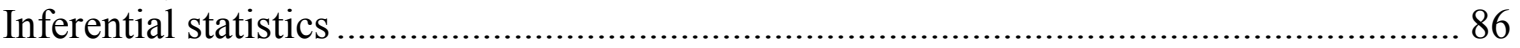

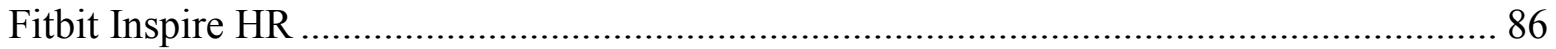

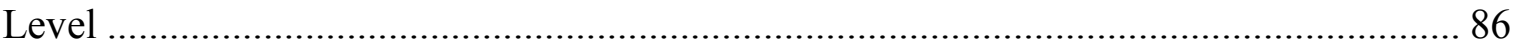

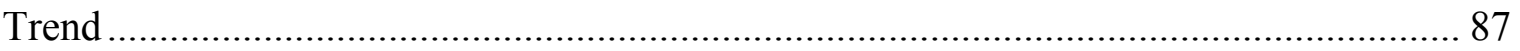

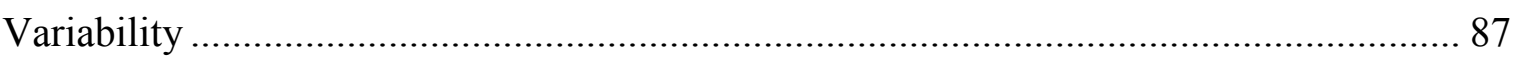

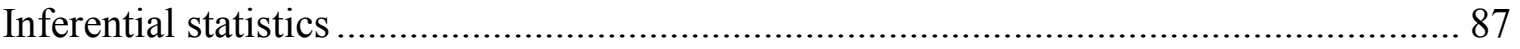

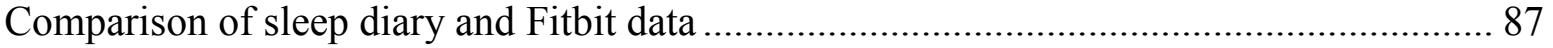

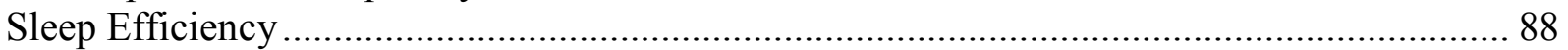

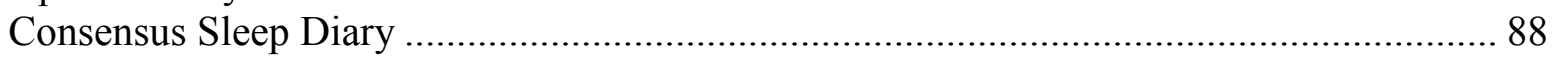

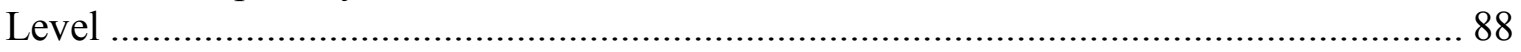

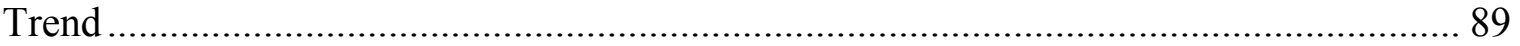

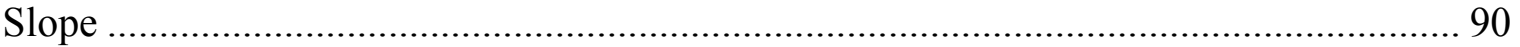

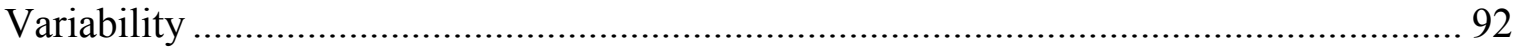

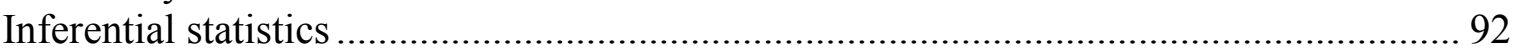

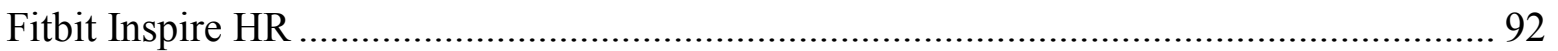

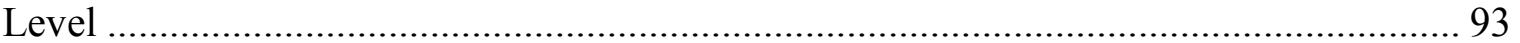

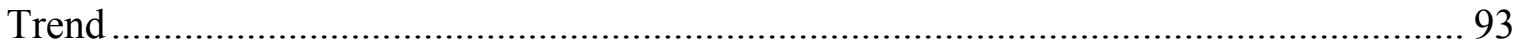

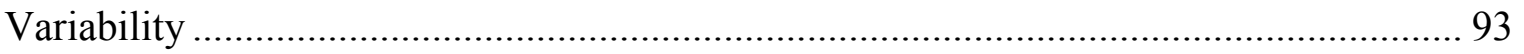

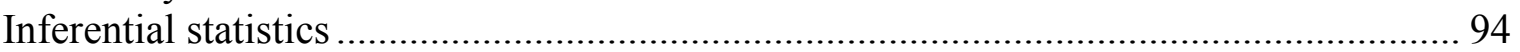

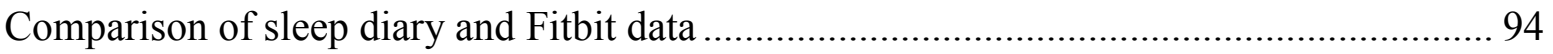

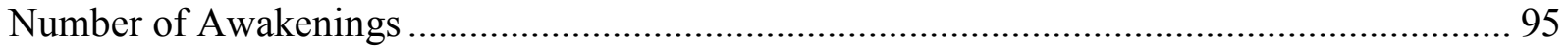

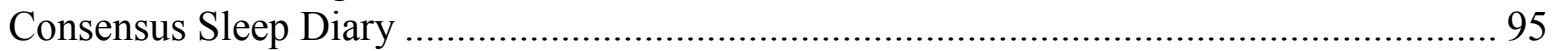




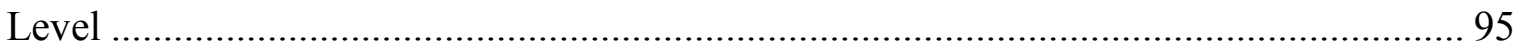

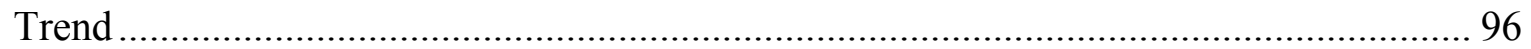

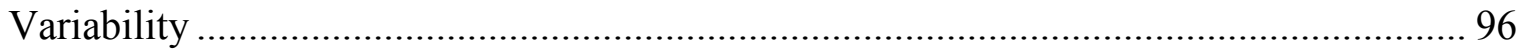

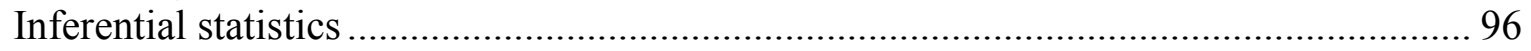

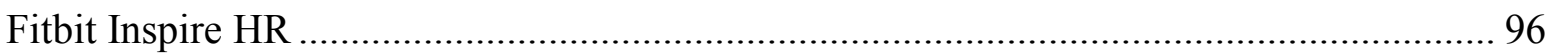

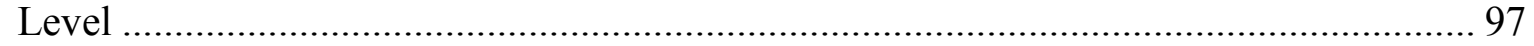

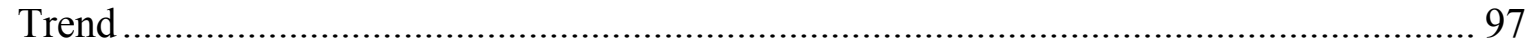

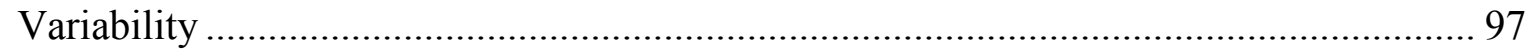

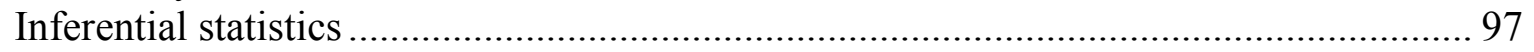

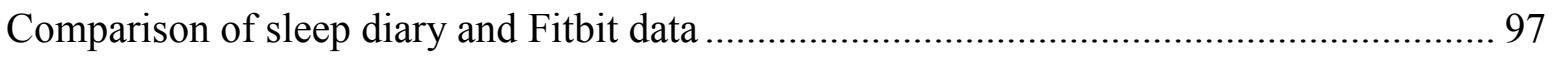

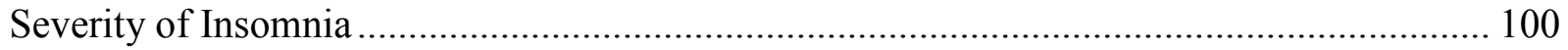

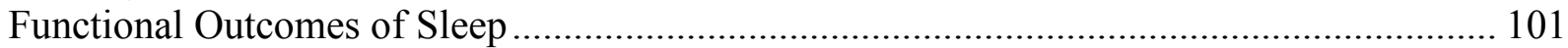

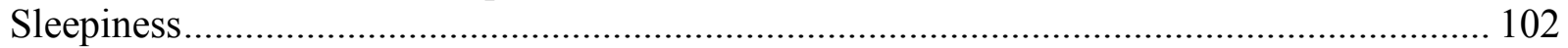

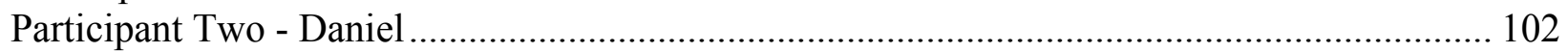

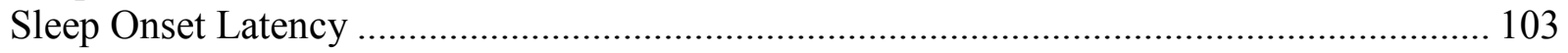

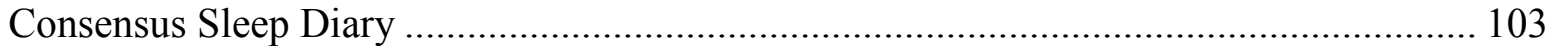

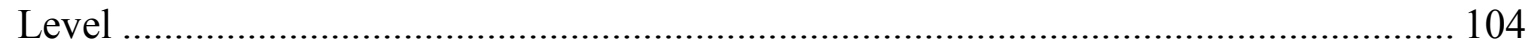

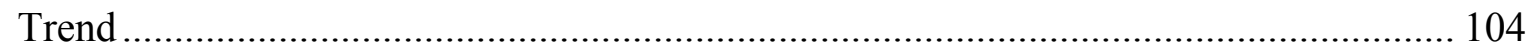

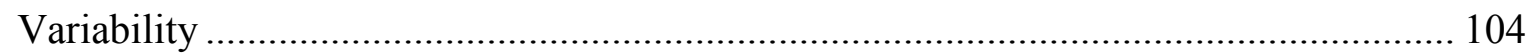

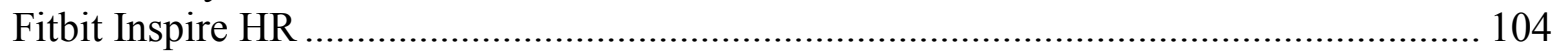

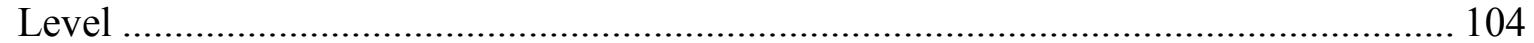

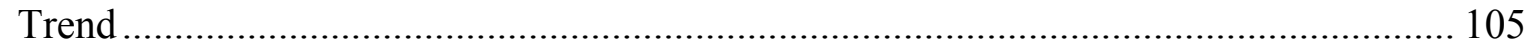

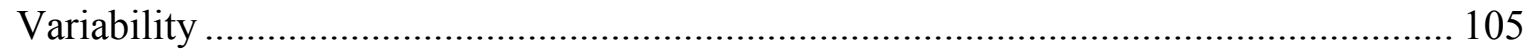

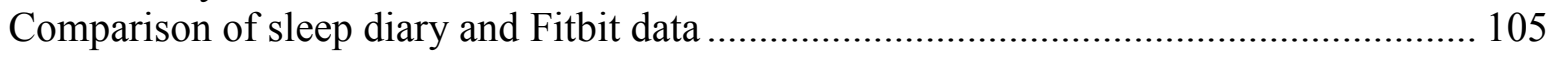

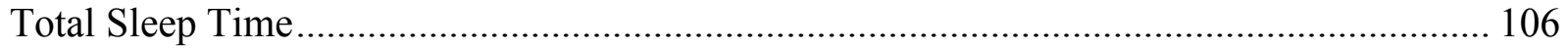

Consensus Sleep Diary ………………………… 106

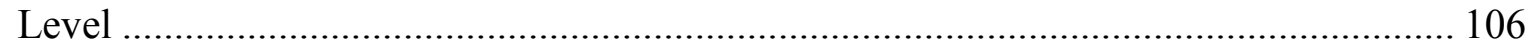

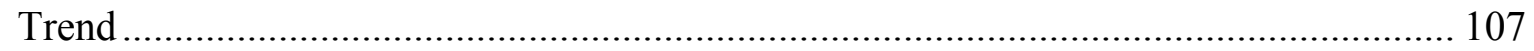

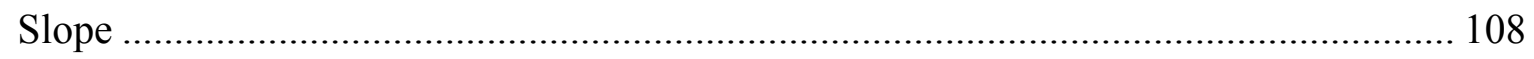

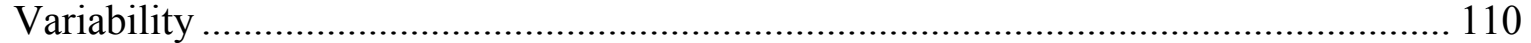

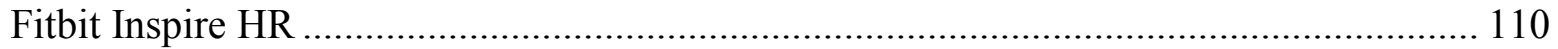

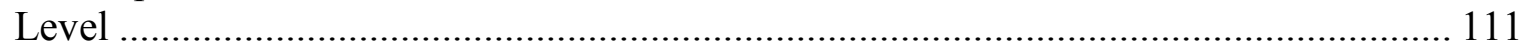

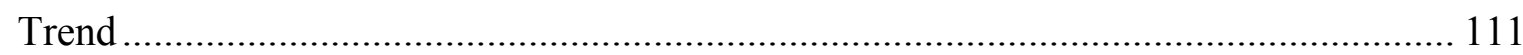

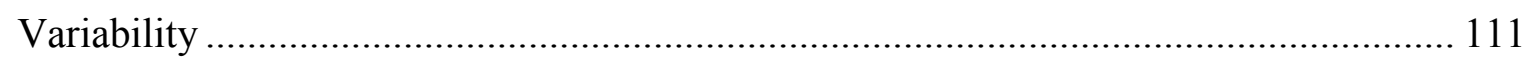

Comparison of sleep diary and Fitbit data ………….................................................... 112

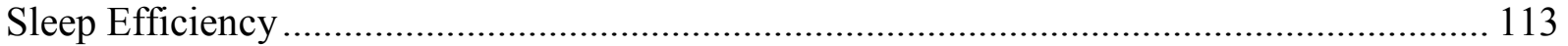

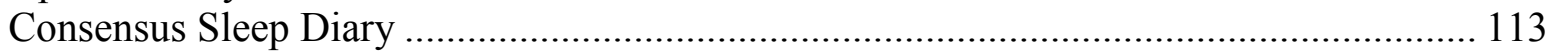

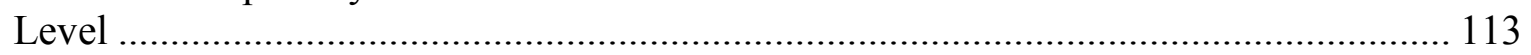

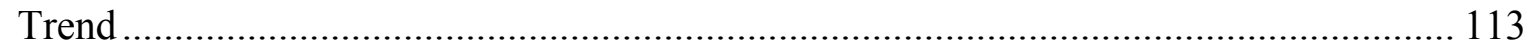

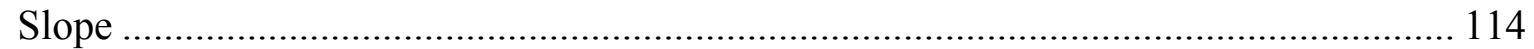

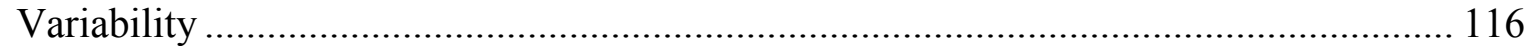

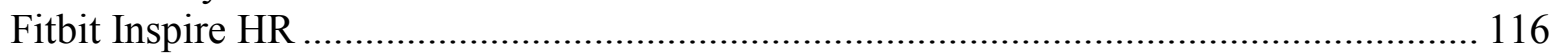

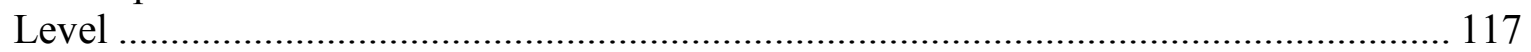

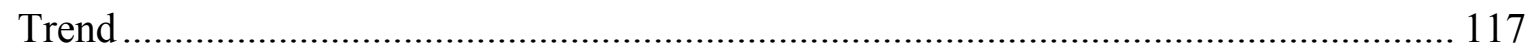

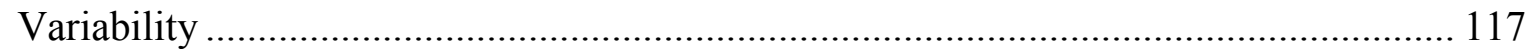

Comparison of sleep diary and Fitbit data ..................................................................... 118 


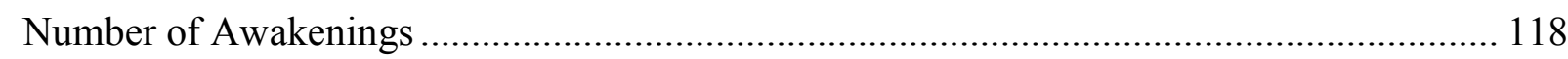

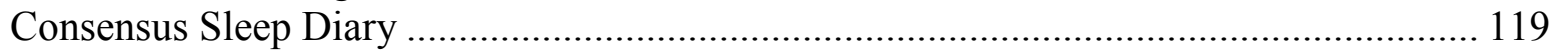

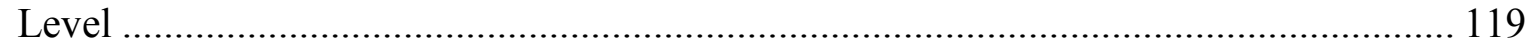

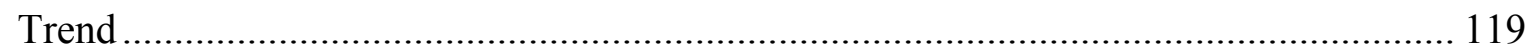

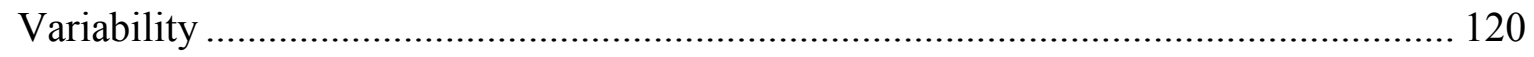

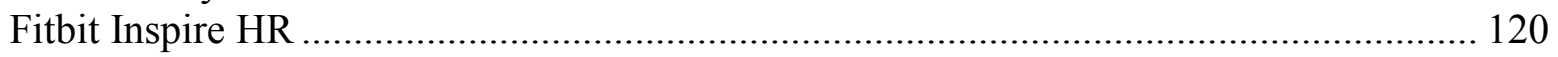

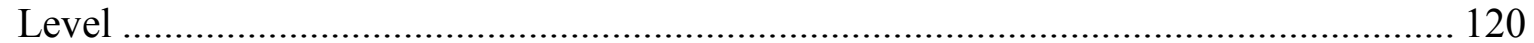

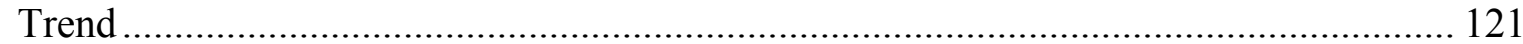

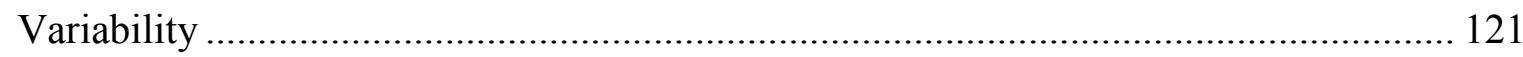

Comparison of sleep diary and Fitbit data …………………...................................... 121

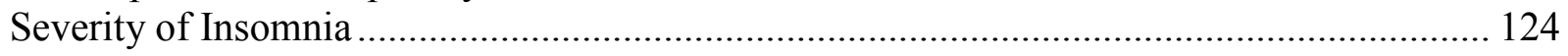

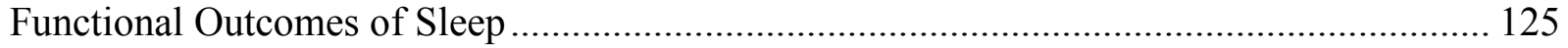

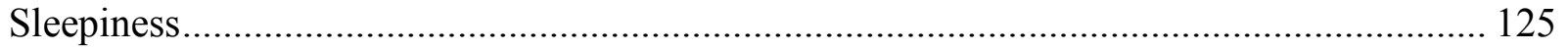

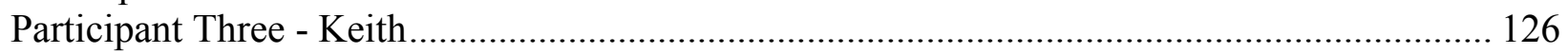

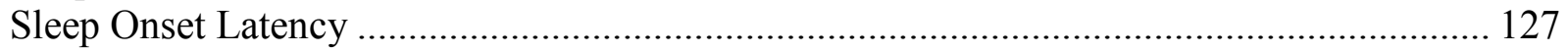

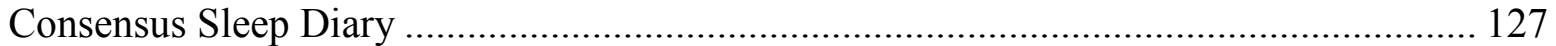

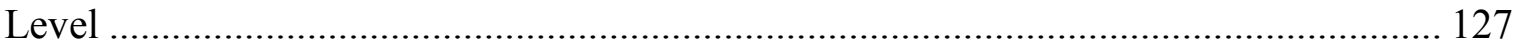

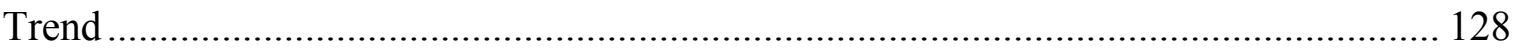

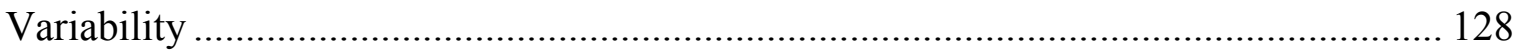

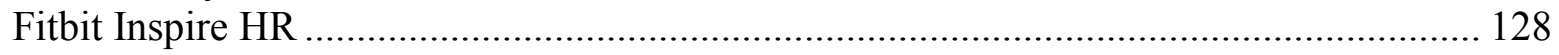

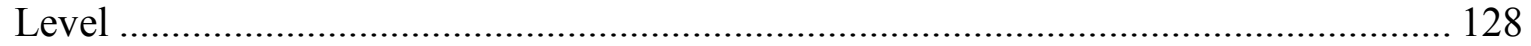

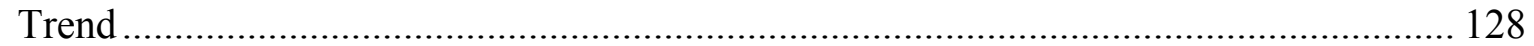

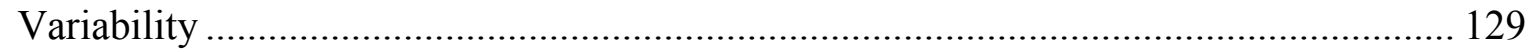

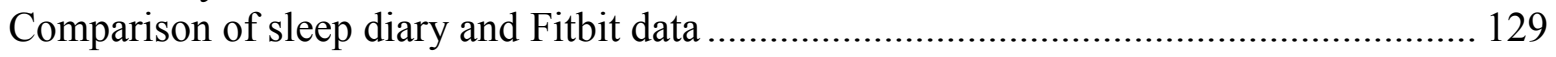

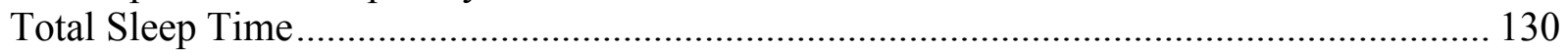

Consensus Sleep Diary ……………………… 130

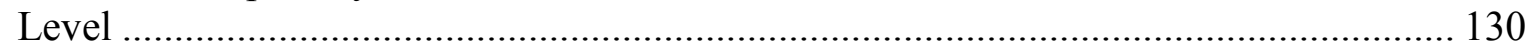

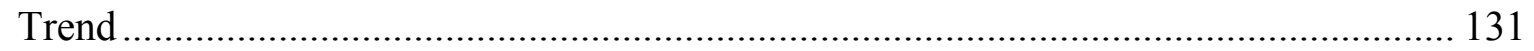

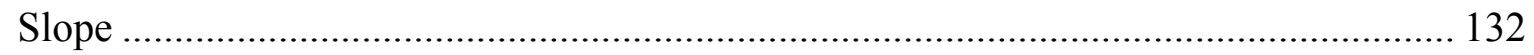

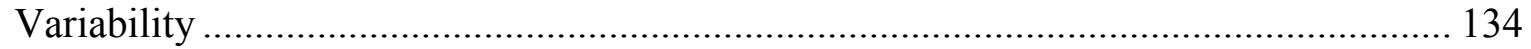

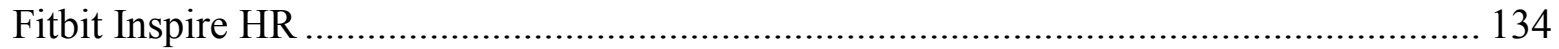

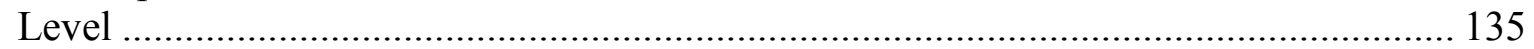

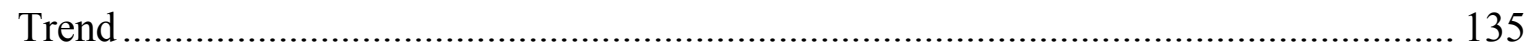

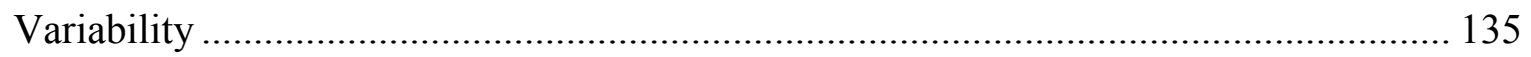

Comparison of sleep diary and Fitbit data ............................................................... 136

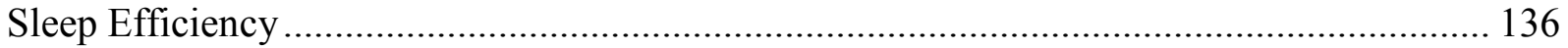

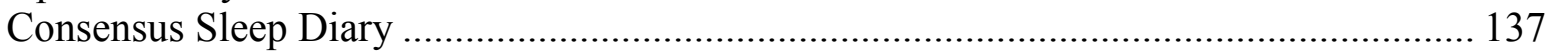

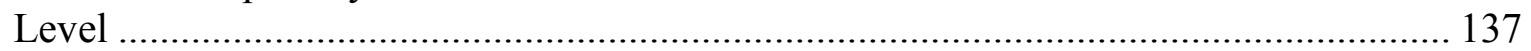

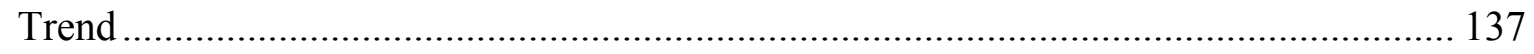

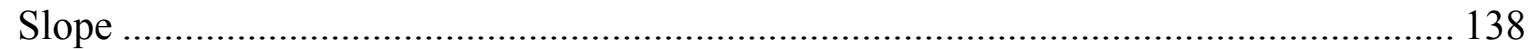

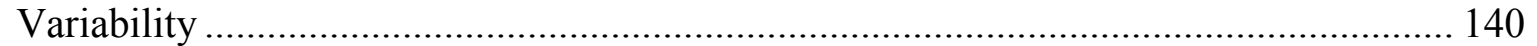

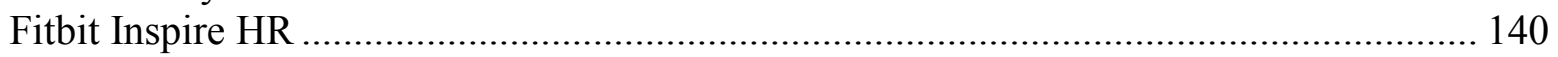

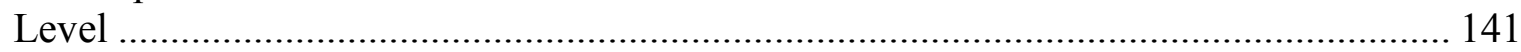

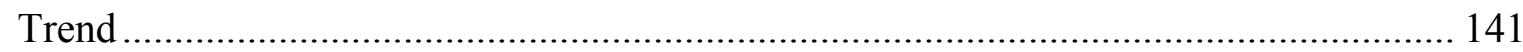

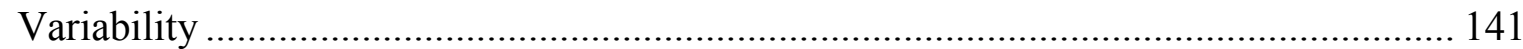

Comparison of sleep diary and Fitbit data .................................................................. 142 


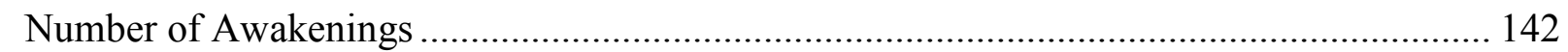

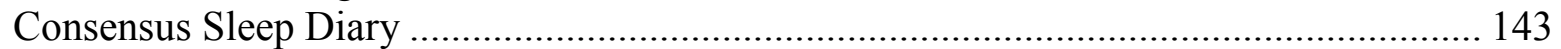

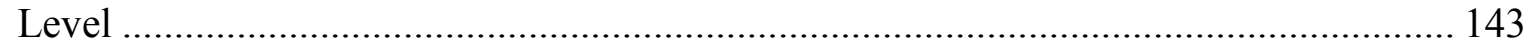

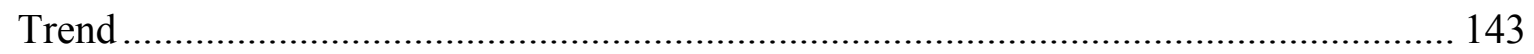

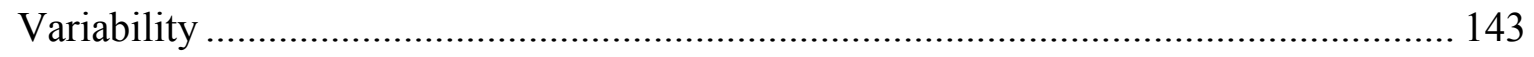

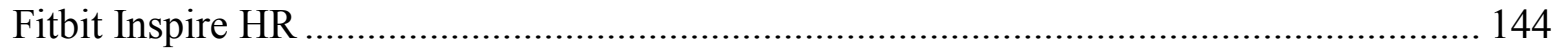

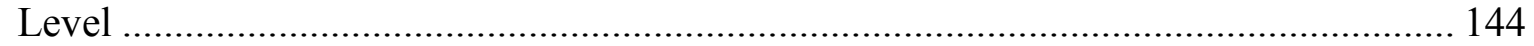

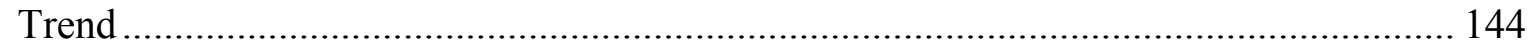

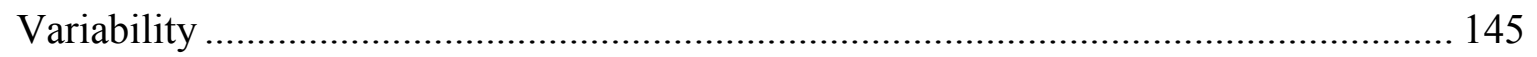

Comparison of sleep diary and Fitbit data ……………............................................. 145

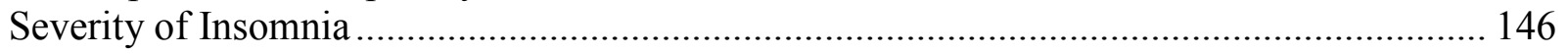

Functional Outcomes of Sleep ……………………….......................................... 148

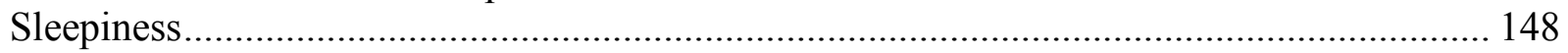

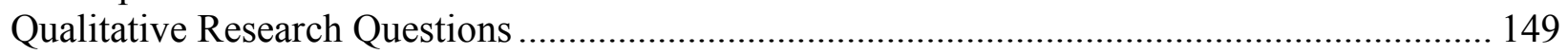

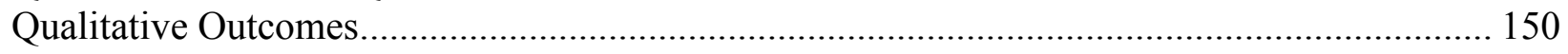

Individual Textural and Structural Descriptions of the Experience....................................... 152

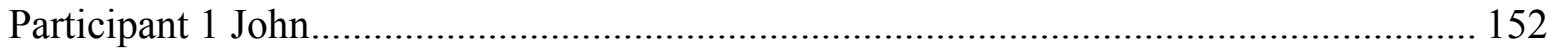

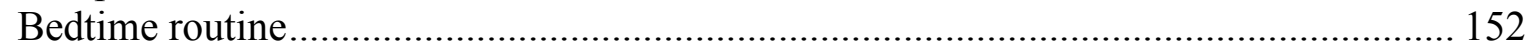

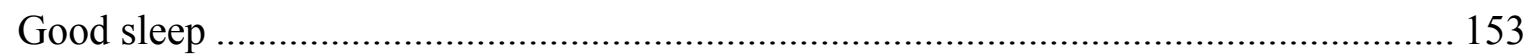

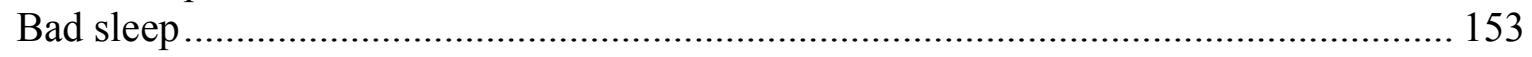

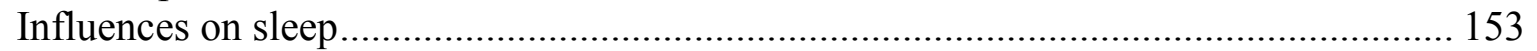

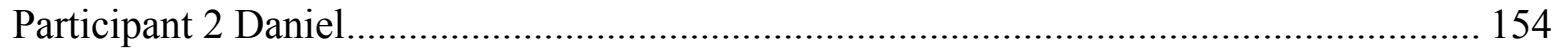

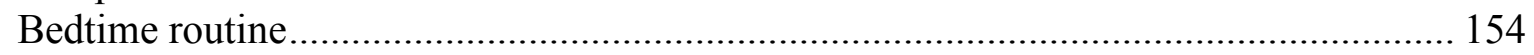

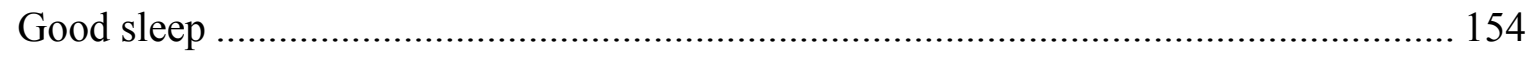

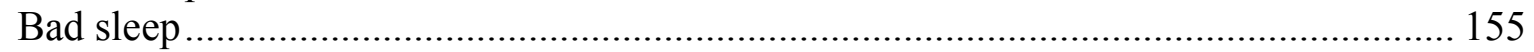

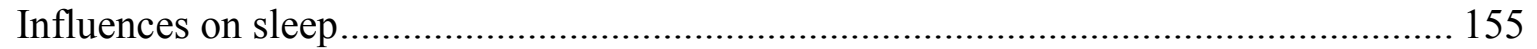

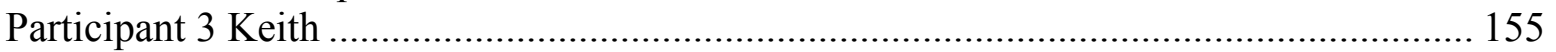

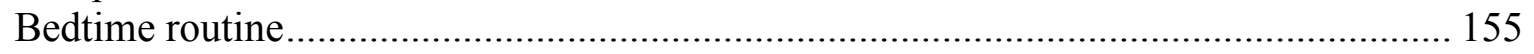

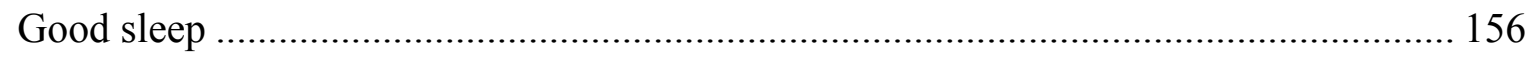

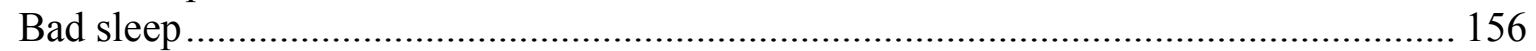

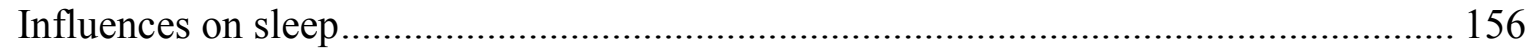

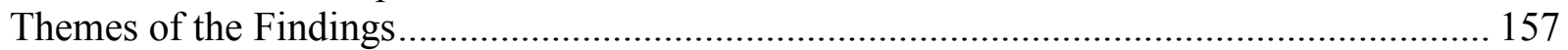

Textural and Structural Description of the Meaning and Essence of the Experience ............. 157

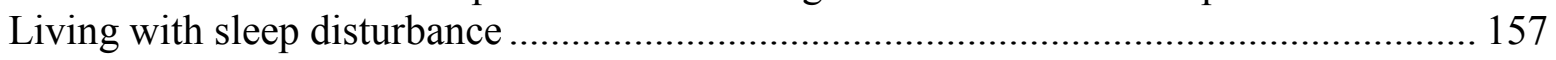

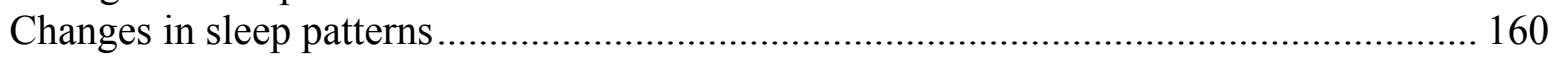

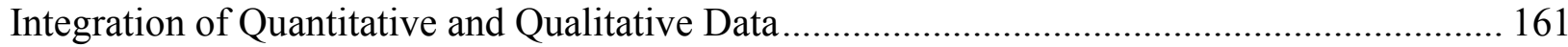

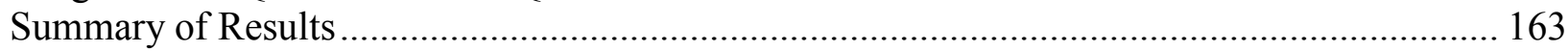

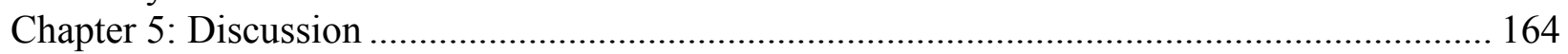

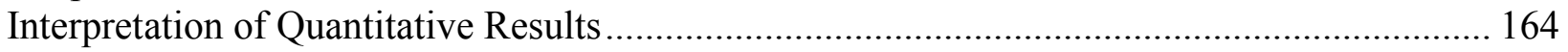

Sleep Participation Outcomes ...................................................................................... 165

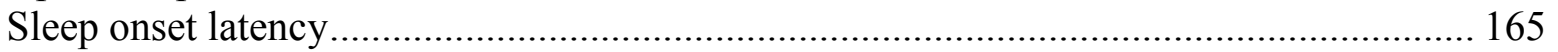

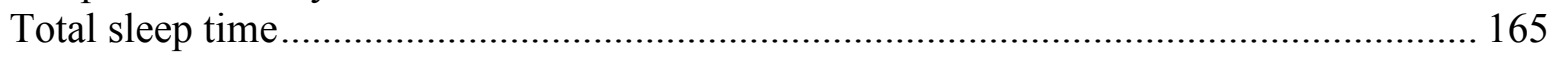

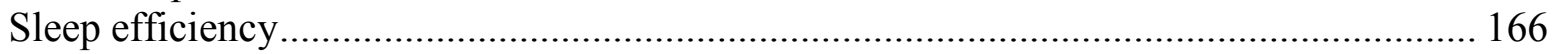

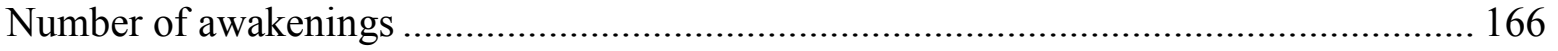

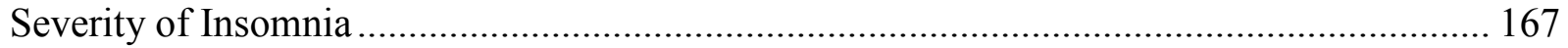

Functional Outcomes of Sleep …………………………............................................ 168 


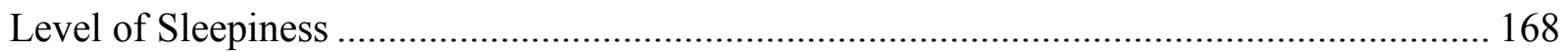

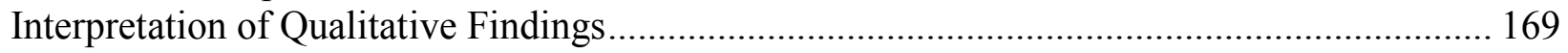

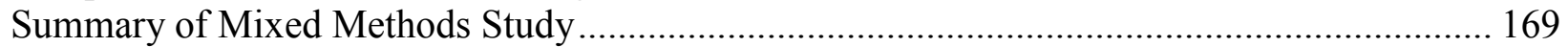

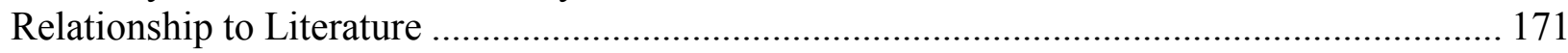

Relationship to Guiding Theoretical Perspectives .................................................................. 175

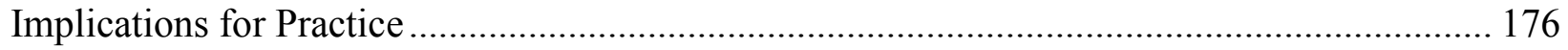

Evaluation of Sleep and Selection of Interventions ......................................................... 177

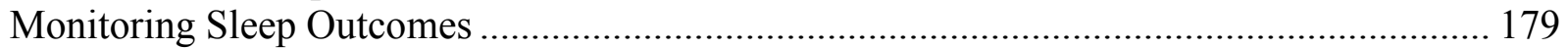

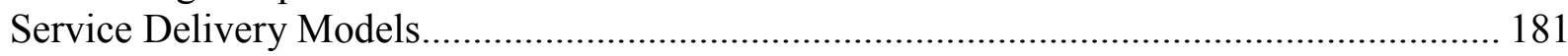

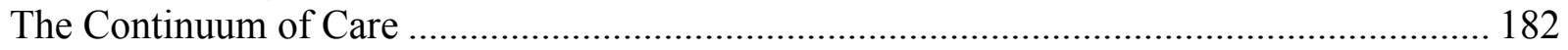

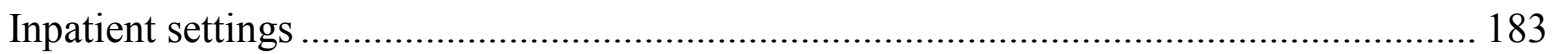

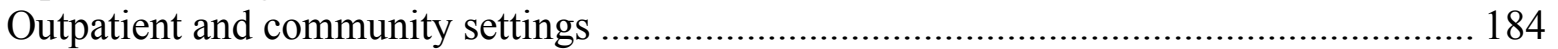

Working with Individuals with Cognitive Impairment................................................... 184

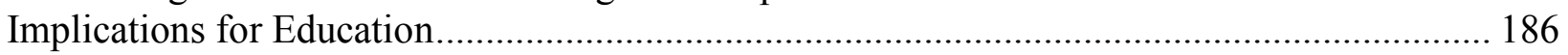

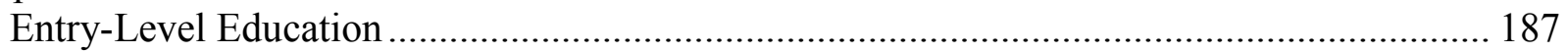

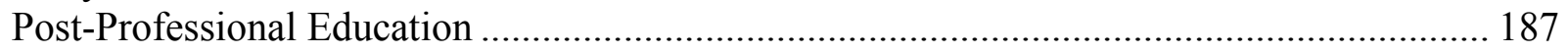

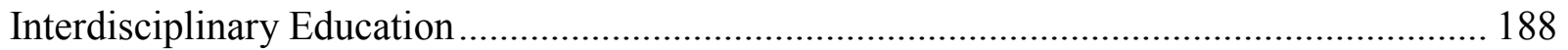

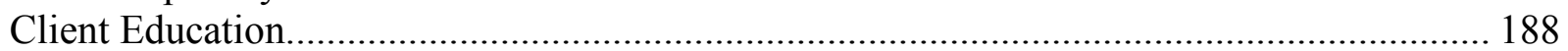

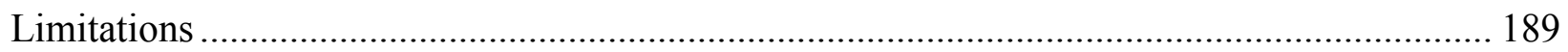

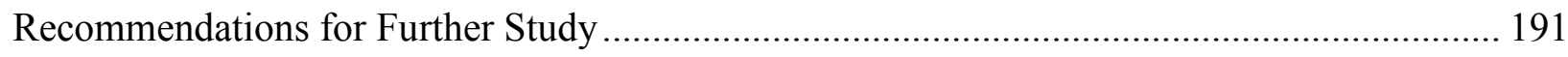

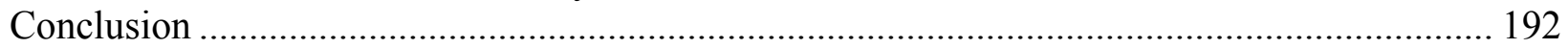

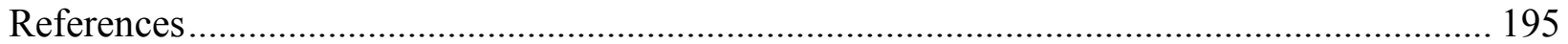

Appendix A: Trail Making Test, Part B Score Sheet................................................................. 209

Appendix B: Insomnia Severity Index Score Sheet................................................................ 211

Appendix C: Sleep Hygiene Index Score Sheet ................................................................ 212

Appendix D: Functional Outcomes of Sleep Questionnaire Score Sheet.................................. 213

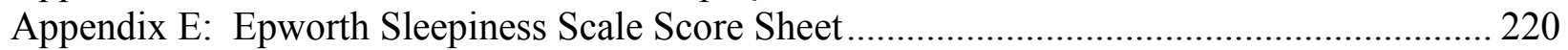

Appendix F: Consensus Sleep Diary Worksheet ................................................................... 221

Appendix G: Sample Fitbit Data Output ……………………………………………… 223

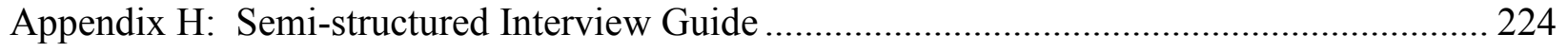

Appendix I: Participant Consent Form ............................................................................ 225

Appendix J: Health Insurance Portability and Accountability Act Form ................................... 229

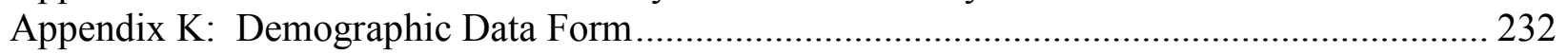

Appendix L: Caregiver Agreement Form ……….......................................................... 233

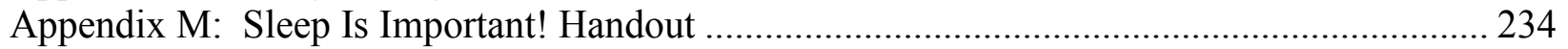

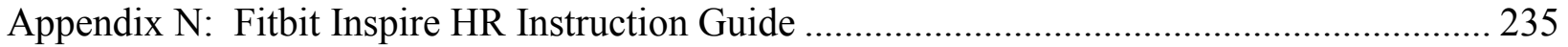

Appendix O: Sample Intervention Daily Checklists................................................................... 236

Appendix P: Intervention Phases Educational Handouts......................................................... 239

Appendix Q: Copyright Permissions ................................................................................ 242 


\section{List of Tables}

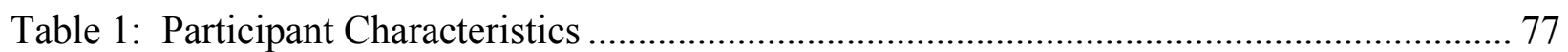

Table 2: Participant One Mean Comparison........................................................................... 99

Table 3: Participant Two Mean Comparison ........................................................................ 123

Table 4: Participant Three Mean Comparison ................................................................. 147

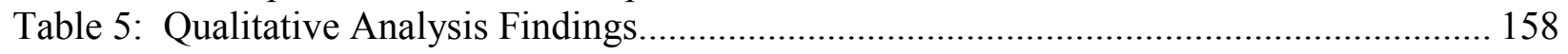

Table 6: Integration of Quantitative and Qualitative Data................................................. 162 
List of Figures

Figure 1: The Person-Environment-Occupation Model of Occupational Performance ............... 35

Figure 2: Detailed Person-Environment-Occupation Model of Occupational Performance ........ 36

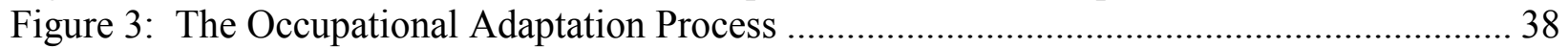

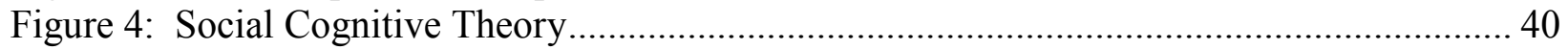

Figure 5: Graphic Depiction of the Embedded Mixed Methods Design .................................... 49

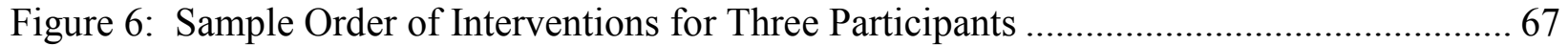

Figure 7: Participant One Sleep Diary Sleep Onset Latency …………………......................... 79

Figure 8: Participant One Fitbit Sleep Onset Latency …………................................................ 80

Figure 9: Participant One Combined Data Sleep Onset Latency................................................. 82

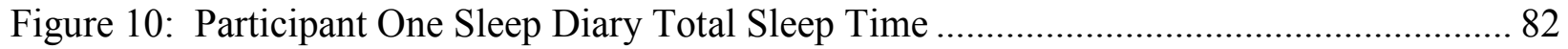

Figure 11: Participant One Total Sleep Time Celeration Line .................................................... 83

Figure 12: Participant One Sleep Diary Total Sleep Time Slope Phase B .................................. 84

Figure 13: Participant One Sleep Diary Total Sleep Time Slope Phase C ................................ 85

Figure 14: Participant One Sleep Diary Total Sleep Time Slope Phase D .................................. 85

Figure 15: Participant One Fitbit Total Sleep Time............................................................ 86

Figure 16: Participant One Combined Data Total Sleep Time .................................................. 88

Figure 17: Participant One Sleep Diary Sleep Efficiency …………………............................ 89

Figure 18: Participant One Sleep Diary Sleep Efficiency Celeration Line ................................ 90

Figure 19: Participant One Sleep Diary Sleep Efficiency Slope Phase B .................................. 91

Figure 20: Participant One Sleep Diary Sleep Efficiency Slope Phase C ................................... 91

Figure 21: Participant One Sleep Diary Sleep Efficiency Slope Phase D ................................... 92

Figure 22: Participant One Fitbit Sleep Efficiency .................................................................... 93

Figure 23: Participant One Combined Data Sleep Efficiency .................................................... 94

Figure 24: Participant One Sleep Diary Number of Awakenings................................................. 95

Figure 25: Participant One Fitbit Number of Awakenings ........................................................... 96

Figure 26: Participant One Combined Data Number of Awakenings ....................................... 98

Figure 27: Participant One Insomnia Severity Index............................................................. 100

Figure 28: Participant One Functional Outcomes of Sleep Questionnaire ................................. 101

Figure 29: Participant One Epworth Sleepiness Scale............................................................ 102

Figure 30: Participant Two Sleep Diary Sleep Onset Latency .................................................... 103

Figure 31: Participant Two Fitbit Sleep Onset Latency........................................................... 105

Figure 32: Participant Two Combined Data Sleep Onset Latency ............................................ 106

Figure 33: Participant Two Sleep Diary Sleep Total Sleep Time................................................ 107

Figure 34: Participant Two Total Sleep Time Celeration Line................................................... 108

Figure 35: Participant Two Sleep Diary Total Sleep Time Slope Phase C ............................... 109

Figure 36: Participant Two Sleep Diary Total Sleep Time Slope Phase D .............................. 109

Figure 37: Participant Two Sleep Diary Total Sleep Time Slope Phase B …………................ 110

Figure 38: Participant Two Fitbit Total Sleep Time................................................................. 111

Figure 39: Participant Two Combined Data Total Sleep Time .................................................. 112

Figure 40: Participant Two Sleep Diary Sleep Efficiency............................................................. 113

Figure 41: Participant Two Sleep Diary Sleep Efficiency Trend .............................................. 114

Figure 42: Participant Two Sleep Diary Sleep Efficiency Slope Phase C................................. 115

Figure 43: Participant Two Sleep Diary Sleep Efficiency Slope Phase D ................................. 115

Figure 44: Participant Two Sleep Diary Sleep Efficiency Slope Phase B................................. 116

Figure 45: Participant Two Fitbit Sleep Efficiency ……………………………...................... 117 
Figure 46: Participant Two Combined Data Sleep Efficiency............................................. 118

Figure 47: Participant Two Sleep Diary Number of Awakenings ........................................ 119

Figure 48: Participant Two Fitbit Number of Awakenings ................................................ 120

Figure 49: Participant Two Combined Data Number of Awakenings................................... 122

Figure 50: Participant Two Insomnia Severity Index ........................................................ 124

Figure 51: Participant Two Functional Outcomes of Sleep Questionnaire ............................ 125

Figure 52: Participant Two Epworth Sleepiness Scale .................................................... 126

Figure 53: Participant Three Sleep Diary Sleep Onset Latency ............................................ 127

Figure 54: Participant Three Fitbit Sleep Onset Latency....................................................... 129

Figure 55: Participant Three Combined Data Sleep Onset Latency ...................................... 130

Figure 56: Participant Three Sleep Diary Total Sleep Time................................................ 131

Figure 57: Participant Three Total Sleep Time Celeration Line............................................. 132

Figure 58: Participant Three Sleep Diary Total Sleep Time Slope Phase D ........................... 133

Figure 59: Participant Three Sleep Diary Total Sleep Time Slope Phase B .......................... 133

Figure 60: Participant Three Sleep Diary Total Sleep Time Slope Phase C ........................... 134

Figure 61: Participant Three Fitbit Total Sleep Time...................................................... 135

Figure 62: Participant Three Combined Data Total Sleep Time ......................................... 136

Figure 63: Participant Three Sleep Diary Sleep Efficiency ................................................. 137

Figure 64: Participant Three Sleep Diary Sleep Efficiency Trend ........................................ 138

Figure 65: Participant Three Sleep Diary Sleep Efficiency Slope Phase D ........................... 139

Figure 66: Participant Three Sleep Diary Sleep Efficiency Slope Phase B............................. 139

Figure 67: Participant Three Sleep Diary Sleep Efficiency Slope Phase C............................ 140

Figure 68: Participant Three Fitbit Sleep Efficiency ......................................................... 141

Figure 69: Participant Three Combined Data Sleep Efficiency ........................................... 142

Figure 70: Participant Three Sleep Diary Number of Awakenings ....................................... 143

Figure 71: Participant Three Fitbit Number of Awakenings .............................................. 144

Figure 72: Participant Three Number of Awakenings ......................................................... 145

Figure 73: Participant Three Insomnia Severity Index ...................................................... 146

Figure 74: Participant Three Functional Outcomes of Sleep Questionnaire .......................... 148

Figure 75: Participant Three Epworth Sleepiness Scale ...................................................... 149 


\section{Chapter One: Introduction}

\section{Background Information}

Sleep is a very important aspect of life, and it consumes approximately one third of a person's time if they are getting adequate sleep on a regular basis. However, many people have difficulty obtaining the desired and necessary amount of sleep to function due to illness, stress, lack of time, or other disruptions in daily life. The American Occupational Therapy Association (AOTA, 2012) reported, "Restful and adequate sleep provides the foundation for optimal occupational performance, participation, and engagement in daily life" (p. 2). However, sleep is an area that is often under-represented in the occupational therapy literature and in occupational therapy practice. While research in the area of sleep is growing in occupational therapy, standard occupational therapy textbooks have historically overlooked sleep (Green, 2015), thus leaving many occupational therapists unaware of their potential role in the promotion of healthy sleep habits.

Several scholarly articles and studies highlight the relationship between sleep and functional performance, participation in other activities of daily living (ADL), quality of life, and overall health and well-being (Duclos, Beauregard, Bottari, Ouelett, \& Gosselin, 2015; Gentry \& Loveland, 2013; O’Donoghue \& McKay, 2012), thus offering a need and an opportunity that can be addressed by occupational therapy practitioners and researchers. While occupational therapists do not diagnose sleep disorders or prescribe medications to improve sleep performance (Tester \& Foss, 2018), they can offer many non-pharmacological interventions to improve sleep quality in a variety of settings and, ultimately, help improve daily functioning and overall wellbeing. 
Getting adequate sleep allows individuals to participate in other functional and meaningful activities, and it is during sleep that the body goes through important physical and mental health changes (Gentry \& Loveland, 2013). However, it is estimated that as many as $50 \%$ of Americans are not getting sufficient sleep on a regular basis (Richardson, 2007). Abnormal sleep has been linked to morbidity and mortality, and chronic deprivation in sleep often negatively affects mental and physical health, can lead to exacerbation of chronic illnesses (Wheaton, Liu, Perry, \& Croft, 2011), and can lead to pre-clinical disability (Lorenz, Budhathoki, Kalra \& Richards, 2014). Impairments with driving, managing finances, performing job duties, and participating in leisure activities often occur with increased fatigue (Wheaton et al., 2011). While insufficient sleep is a serious problem population-wide, it can have detrimental effects when compounded by health conditions, such as brain injury (BI) (Duclos et al., 2015).

\section{Statement of the Research Problem and Significance of Project}

Disruptions in sleep habits have also been linked to cognitive and psychological impairments, reduced participation in daily activities, and decreased quality of life (Bolge, Doan, Kannan, \& Barran, 2009, as cited in Duclos et al., 2015). A study conducted by Duclos et al. (2015) highlighted the potential limitations caused by disruptions in sleep and the potential role that occupational therapists have in regards to promoting improved sleep habits after BI.

However, there are minimal studies documented in the literature that support the effectiveness of occupational therapy interventions in relationship to healthy sleep habits (Gutman et al., 2017; Smallfield \& Lucas Molitor, 2018). Therefore, a study designed to understand the effectiveness of various non-pharmacological sleep interventions for individuals after BI from an occupational 
therapy perspective would have many potential benefits for clients and would add to the growing body of literature of multidisciplinary sleep intervention studies.

Several studies in various disciplines have examined the effectiveness of nonpharmacological sleep interventions (Bogdanov, Naismith, \& Lah, 2017; Leland et al., 2016; Neuendorf et al., 2015; Wang, Ying Chair, Mi Ling Wong, \& Li, 2016). Other studies, such as the one conducted by Leland and colleagues (2016), look at how occupation-based interventions affect sleep performance. The authors identified that individuals who participated in occupations, instead of daytime napping, had improved nighttime sleep (Leland et al., 2016). Many other studies and scholarly articles have also identified the potential role of occupational therapy in the promotion of healthy sleep habits but have neglected to provide evidence supporting the effectiveness of such interventions (Fung, Wiseman-Hakes, Stergiou-Kita, Nguyen, \& Colantonio, 2013; Gentry \& Loveland, 2013; Leland, Marcione, Schepens Niemiec, Kelkar, \& Fogelberg, 2014). The acknowledgment of the role of occupational therapy opens the door for future studies to examine the effectiveness of various sleep interventions from an occupational therapy perspective and to attempt to understand the occupational implications of disrupted sleep.

\section{Relevance to Occupational Therapy}

Fung et al. (2013) highlighted the role of occupational therapy in the promotion of healthy sleep habits by stating that, "given their skills in relation to the assessment of the personal and environmental issues influencing sleep, occupational therapists are well positioned to enhance their roles in the area of sleep and sleep problems" (p. 385). For example, Fung et al. suggested that occupational therapists should evaluate and recommend appropriate environmental and behavioral changes in order to enhance sleep performance. Coaching and 
assisting clients in tracking sleep performance and educating clients on appropriate techniques to improve sleep are other potential roles for occupational therapy (Gentry \& Loveland, 2013).

A study examining the effectiveness of various sleep interventions for individuals after BI would help to support occupational therapy's role in identifying sleep deficits and plausible solutions to improve sleep performance, which will ultimately improve clients' occupational performance and overall quality of life. Additionally, it would allow for the exploration of the unique perspectives of clients in regards to the occupational implications of sleep disturbances for individuals after BI. The design of this study could then be applied to other populations and in other settings, allowing occupational therapists to promote healthy sleep habits with a variety of clients with deficits in sleep performance. The use of established theoretical frameworks should also be used in order to guide the development and implementation of a study related to sleep.

\section{Relevant Theoretical Frameworks}

For the current study, the Person-Environment-Occupation (PEO) model, Occupational Adaptation (OA), and the Social Cognitive Theory (SCT) were used to guide the development and implementation of sleep interventions. The PEO model, OA, and the SCT each focus on the interaction between factors related to the person, the environment, and occupations or behaviors. Additionally, the frameworks look at how these factors affect a functional outcome or behavior change, which would be useful for promoting increased sleep performance for individuals after BI through the implementation of suggested personal, environmental, and occupational sleep interventions. The development of the current study was also guided by the following research questions. 


\section{Research Questions}

The current research study was a mixed methods study, with both quantitative and qualitative research questions. There were two quantitative and two qualitative questions, which are listed below. These research questions were addressed during various stages of the mixed methods study, and results of the study should provide preliminary findings to answer these research questions.

\section{Quantitative Research Questions}

1. Does participation in a multicomponent sleep program improve sleep participation for adults after BI who are participating in a day treatment community re-entry program?

2. Which sleep interventions, if any, are effective for improving sleep participation for this population?

\section{Qualitative Research Questions}

1. What factors affect sleep participation for each participant in the study?

2. What are the occupational implications of decreased sleep after BI?

\section{Operational Definitions}

The following definitions are presented to depict how concepts are defined within the context of the study.

Environmental intervention involves modifications to various aspects of the external physical and/or social conditions that surround a client (AOTA, 2014).

Functional participation is involvement in purposeful and useful life tasks, activities, or situations. 
Non-pharmacological sleep interventions or sleep interventions are activities that are intended to improve sleep participation through behavioral, task-related, or environmental modifications that do not include medicinal treatments.

Occupation-based intervention involves modifications of the life activities that a person engages in.

Person-based intervention is an activity or treatment that is designed to change client factors that negatively affect performance.

Pharmacological sleep interventions are interventions to improve sleep and involve the use of sleep medication.

Sleep diary is a personal record of sleep-related factors and activities.

Sleep efficiency is the percentage of the total time spent asleep in minutes divided by the total amount of time in bed in minutes.

Sleep hygiene involves behaviors or other sleep-related factors that are used to support improved sleep quality and quantity (Stepanski \& Wyatt, 2003).

Sleep onset latency is the amount of time it takes to go from being awake to falling asleep in minutes.

Sleep participation is the act of sustaining a state of sleep without disruption (AOTA, 2014).

Sleep performance is the accomplishment of sleep-related activities.

Sleep preparation involves the habits and routines related to preparing for sleep (AOTA, 2014).

Sleep tracker is a device that is used to monitor, measure, and/or record changes in sleep patterns and sleep performance. 
Stimulus control therapy (SCT) involves practices that create and reinforce a positive association between the bedroom and sleep and allow for falling asleep.

Total sleep time is the total amount of time, in minutes, spent actually sleeping

\section{Summary of Introduction Chapter}

This chapter provided background information related the importance of sleep, the research problem, the relevance to occupational therapy and applicable theories to guide the research, research questions and operational definitions for the proposed study. The next chapter will give an overview of the constructs of the study, a more in-depth explanation of applicable guiding theories, and a review of existing literature related to sleep and applicable methodologies for sleep intervention studies. 


\section{Chapter Two: Literature Review}

As discussed in the previous chapter, sleep is a very important part of life that helps to support active engagement in other aspects of daily living. Prior to conducting any study related to sleep, it is important to understand the physiology of sleep, which factors support or hinder healthy sleep habits and sleep hygiene, and what sleep typically looks like after BI.

Additionally, it is necessary to have an understanding of the factors that support improved sleep participation after $\mathrm{BI}$ and to be familiar with the overall process and occurrence of BI prior to implementing sleep interventions for this population.

\section{Overview of Brain Injury}

Acquired brain injury (ABI) occurs after birth and results from a change to the brain's neuronal activity, thus affecting the nerve cells in the brain (Brain Injury Association of America [BIAA], n.d.). An ABI can either be traumatic or non-traumatic. A traumatic brain injury (TBI) is caused by an external force, and a non-TBI is caused by an internal force that creates an alteration in brain cells and brain function (BIAA, n.d.; Shepherd Center, 2018). Because nonTBIs affect the brain at a cellular level, the injury has the ability to spread to other areas of the brain (Shepherd Center, 2018). The severity of injuries can vary from mild to severe and can result in short-term or long-term deficits (Shepherd Center, 2018), and patterns of dysfunction depend on what part of the brain is affected by the injury (BIAA, n.d.).

The brain is separated into different lobes, which are responsible for various brain and bodily functions. The frontal lobe is responsible for emotional and impulse control and behavioral functions, and the temporal lobe controls communication and memory (BIAA, n.d.). The parietal lobe controls the five primary senses, and the brainstem is responsible for the body's involuntary functions, such as breathing and heart rate (BIAA, n.d.). The cerebellum affects 
balance, movement, and coordination, and the occipital lobe affects vision (BIAA, n.d.). Injuries to the brain will manifest into deficits that relate to the specific functions of the affected lobe. The lobes of the brain are also categorized as either part of the right or left side of the brain, with each side being responsible for different functions.

The left side of the brain supports analytical, logical, and organized thought, whereas the right side controls more creative, intuitive, empathetic, and figurative traits (BIAA, n.d.). Injuries that occur on the left side of the brain often create difficulties in receptive and expressive language, impaired logic, sequencing deficits, increased depression and anxiety, and decreased control over the right side of the body (BIAA, n.d.). Injuries on the right side of the brain can cause visual-spatial deficits, inattention to the left side of the body, decreased awareness, visual memory problems, altered creative thinking, and decreased control over the left side of the body (BIAA, n.d.). The prevalence of BI is often associated with certain risk factors.

\section{Prevalence of Brain Injury}

The Centers for Disease Control and Prevention (CDC, 2016) reported that there were approximately 2.8 million emergency department visits, hospitalizations, and deaths as a result of TBI in 2013, with TBI contributing to about $30 \%$ of all deaths related to injury. Falling, being struck by or against an object, motor vehicle accidents, and self-harm are noted to be the leading causes of TBI (BIAA, n.d.; CDC, 2016; Shepherd Center, 2018), whereas stroke, brain infections, lack of oxygen, tumors, and drug overdose are common causes of non-TBIs (BIAA, n.d.; Shepherd Center, 2018). Common risk factors for TBI include being 75 years of age and older (CDC, 2016), driving while under the influence of drugs or alcohol or while using a cellphone, and not taking necessary precautions during risky or potentially dangerous sports and activities (Shepherd Center, 2018). Common risk factors for stroke include high blood pressure, 
diabetes, heart disease, smoking, personal or familial history of stroke, alcohol and illegal drug use, lack of exercise and obesity, and a unhealthy diet (U.S. Department of Health and Human Services, 2018). Minimizing these risk factors can help to avoid the devastating functional impairments that often occur as a result of $\mathrm{BI}$.

\section{Functional Implications of Brain Injury}

It is estimated that over three million Americans need long-term assistance with ADLs after TBI, and TBI can cause a wide range of other functional deficits, including changes with thinking and learning, emotions and behaviors, and language (Shepherd Center, 2018). TBI can also cause epilepsy and increase the risk of other brain disorders, such as Alzheimer's and Parkinson's disease (Shepherd Center, 2018). Depending on the severity of the BI, effects might include a temporary loss of consciousness, respiratory problems, and impaired motor functions (BIAA, n.d.). Various neurological symptoms, such as irritability, aggression, and disorientation, might occur but often subside as the brain and other body systems become more stable (BIAA, n.d.). Because the neurons in the brain do not mend themselves, damage and functional impairments may be lifelong after BI (BIAA, n.d.). In fact, many survivors of BI have lasting cognitive, emotional, and physical deficits (Howrey, Graham, Pappadis, Granger, \& Ottenbacher, 2017) that affect their overall well-being and ability to participate in meaningful life activities. Functional recovery after BI is variable depending on the person and individual's activities during the recovery process.

\section{Functional Recovery after Brain Injury}

During the recovery process, individuals who are post-BI should avoid activities that are physically demanding or that could increase the risk of additional injury (CDC, 2016). Individuals should gradually return to normal activities, as able, and it may be beneficial to make 
adjustments that simplify or add more structure to daily routines due to cognitive or physical deficits (CDC, 2016). Additionally, it is important for people after BI to get adequate rest and sleep to support optimal recovery and functional engagement in life activities.

Functional recovery after BI depends on many factors, including the severity of the injury, the age of the person injured, and variations in post-acute care (Howrey et al., 2017). Many individuals after BI pursue rehabilitation to achieve functional improvements that exceed those of spontaneous recovery alone (Anke et al., 2015). Even though inpatient rehabilitation after BI is associated with increased recovery, several factors affect these functional outcomes.

Anke et al. (2015) found that two thirds of patients after TBI had improved functional outcomes between 3 and 12 months after injury and emphasized the importance of early initiation of rehabilitation after BI. This study, along with others, identified increased age as being associated with decreased motor gains after BI (Anke et al., 2015; Arbour et al., 2017; Howrey et al., 2017), but also found that older age predicts greater life satisfaction after BI (Anke et al., 2017). Life satisfaction was also not influenced by the severity of the BI, but decreased life satisfaction was related to symptoms of depression and anxiety (Anke et al., 2017). While older adults show similar levels of psychological resilience as younger adults after BI, older adults were less likely to be reintegrated into work or school after BI (Arbour et al., 2017) even though Fabiano and Sharrard (2017) found that most people after mild TBI return to work. The Glasgow Coma Scale is used to rate the severity of a brain injury (Fabiano \& Sharrard, 2017), and the Glasgow Outcome Scale-Revised is one of the most commonly used tools to assess functional recovery after BI, with improved scores being linked to an increased capacity to return to pre-injury functional status (Arbour et al., 2017). As previously mentioned, an important part of functional recovery after BI involves sleep. 


\section{Overview of Sleep}

Sleep is a complicated neurological process involving daily cycles that fluctuate between stages of altered consciousness and arousal (Sasaki, 2012). For healthy individuals, sleep cycles typically occur in 90 to 100-minute increments between four and six times a night (Duclos et al., 2015). There are three basic stages of consciousness, which includes wakefulness, non-rapid eye movement (NREM) sleep, and rapid eye movement (REM) sleep (National Sleep Foundation [NSF], n.d.).

\section{Physiology of Sleep}

NREM sleep involves reduced physiological activity, including slowed breathing and heart rate, and decreased blood pressure (NSF, n.d.). Brain waves slow down and gain amplitude as sleep deepens (NSF, n.d.). NREM sleep has three stages. NREM N1 is when people transition from being awake to falling asleep, and reactivity to external stimuli decreases (NSF, n.d.). N1 sleep is considered very light sleep and typically lasts between 1 and 7 minutes after falling asleep (Green \& Wilson, 2015). During NREM N2, people are in a light sleep with periods of muscle tone versus relaxation and decreased heart rate and body temperature (NSF, n.d.). Brain waves become slower with occasional incidents of rapid waves, called sleep spindles (NSF, n.d.). N2 sleep occurs for about half of the night, and it is easy to wake people from this stage of sleep (Green \& Wilson, 2015). NREM N3 is called slow wave sleep (SWS) and involves mostly slow brain waves with occasional smaller and faster waves (NSF, n.d.). This stage is the deepest sleep and involves slow breathing, decreased blood pressure and temperature, and decreased muscle activity (NSF, n.d.). The onset of this stage typically occurs 15 to 45 minutes after falling asleep (NSF, n.d.). When a person wakes up during this state, he or she will likely be disoriented for a short period of time (Green \& Wilson, 2015). Most SWS 
sleep occurs during the first third of the night, where REM sleep typically occurs in the last third of the night (NSF, n.d.).

REM sleep is an active period of sleep, involving fast and desynchronized brain waves, rapid and shallow breathing, increased heart rate and blood pressure, and temporarily paralyzed limb muscles (NSF, n.d.). This state typically occurs after approximately 85 minutes of NREM sleep, and this is when dreams often occur (NSF, n.d.). The first sleep cycle includes the time between the initial onset of sleep and the end of the first REM period, and subsequent cycles start with NREM sleep and end with REM sleep (NSF, n.d.). Each of the sleep stages are equally important, and having the correct balance between the states promotes the most restful and restorative sleep (NSF, n.d.). Certain biological influences also affect sleep and wake cycles.

To help regulate sleep and wake cycles throughout the day, there are wake-promoting centers and sleep-producing centers in the brain (NSF, n.d.). The wake-promoting center is controlled by activity in the brain stem and hypothalamus and is affected by a person's circadian rhythm during the day (NSF, n.d.). The sleep-promoting center is under homeostatic influence, where the drive to sleep increases the longer the person is awake (NSF, n.d.). During sleep, neurons in the hypothalamus inhibit the regions of the brain that promote wakefulness (NSF, n.d.). The bidirectional switch between wakefulness and sleep occurs when one of these systems inhibits the other (NSF, n.d.). For optimal daily functioning, it is important that people are able to regularly go through this alternating process between periods of wakefulness and sleep participation.

\section{Sleep Participation}

Sleep participation occurs when a person sustains a state of sleep without disruption (AOTA, 2014). The process of sleep participation also involves the actions that a person takes in 
order to take care of his or her personal needs for sleep (AOTA, 2014). Being able to participate in sleep is important for physiological functioning and active engagement in life. The NSF (n.d.) reports that sleep influences all of the body's major systems and affects a person's mental health and overall well-being.

Gentry and Loveland (2013) acknowledged that sleep is a very important part of life, and with sufficient amounts of sleep, people are able to participate in other functional and meaningful activities, including ADL, work, leisure interests, and health management. The ability for people to participate in meaningful occupations, including sleep, affects their overall well-being and survival (O’Donoghue \& McKay, 2012). In fact, sleep is necessary to ensure that human beings live a balanced and healthy life (O’Donoghue \& McKay, 2012), which includes adequate immune function, cognitive and learning abilities, regulation of emotions, and occupational performance (Fung et al., 2013). It is during deep sleep that the body goes through very important physiological and psychological changes, such as healing, muscle and tissue growth (Gentry \& Loveland, 2013), rest, and restoration (Tester \& Foss, 2018). AOTA (2012) reported, "Restful and adequate sleep provides the foundation for optimal occupational performance, participation, and engagement in daily life" (p. 2). AOTA (2008) also recognized the importance of sleep, designating sleep as an occupation in the second edition of the Occupational Therapy Practice Framework (OTPF).

\section{Sleep as an Occupation}

AOTA (2014) reported that rest and sleep are "activities related to obtaining restorative rest and sleep to support healthy, active engagement in other occupations" (p. S20). While sleep was originally classified as an ADL in the OTPF, this shift in focus highlights the important role that sleep has on participation in daily life (Gentry \& Loveland, 2013). Perhaps the increased 
interest in sleep has occurred due to a growth in occupational science and the study of human time usage (Green, 2015).

Green (2008) acknowledged the role of occupational science by examining sleep in relationship to time usage, and he implies that sleep supports more than just restoration. Wilcox (1998) studied the science of sleep and argued that sleep is essential for occupational engagement. She also acknowledged the potential relationship between sleep patterns and other occupations (Wilcox, 1998). Charles Christiansen (1996, as cited in Green 2015) also explored the importance of sleep in the 1990s by looking at balance and time use and acknowledged the influence of environmental and social factors on circadian rhythms. Whether, or not, sleep classifies as an occupation has been previously debated in the literature (Green, 2008), but it is evident that healthy sleep habits are important for participating in other areas of occupation and daily life.

\section{Healthy Sleep Habits}

The need for sleep varies from individual to individual and across the lifespan, but most adults sleep somewhere between seven and nine hours per night to achieve adequate daily functioning (NSF, n.d.). Not all sleep habits promote good sleep for every individual, but there are certain practices and behaviors that are widely recognized in the sleep literature to support good sleep participation. For instance, a person should sleep as much as needed to feel rested and healthy the following day, but should not sleep more than that (Stepanski \& Wyatt, 2003). People should also have regular and consistent arousal times to promote regular sleep times, and should have a steady amount of daily exercise early in the day to promote improved sleep (Stepanski \& Wyatt, 2003). A light bedtime snack is encouraged to avoid feelings of hunger, but caffeine and alcohol should be avoided in the evening hours (Stepanski \& Wyatt, 2003). A 
person should also never try to sleep, and if falling asleep is difficult, doing something else and then reattempting sleep may be helpful (Stepanski \& Wyatt, 2003). Additionally, the bedroom should always be conducive to sleep (Green \& Hicks, 2015). While not all healthy sleep habits are universal, the specific tasks that one does in order to prepare for sleep are important for improved sleep participation.

AOTA (2014) reported that sleep preparation includes the routines that prepare someone for comfortable rest, which involves the establishment of healthy sleep patterns. Activities such as participation in evening ADL, saying goodnight to loved ones, listening to music or reading to fall asleep, and adjusting the sleep environment prior to bed are a part of the sleep preparation process (AOTA, 2014). While many people participate in similar bedtime routines, it is important to understand that these routines and nighttime patterns are personally unique and are affected by various contextual factors (AOTA, 2014).

\section{Contextual Influences on Sleep}

AOTA (2014) acknowledges that rest and sleep occur in physically, culturally, and temporally constructed contexts. Additionally, the environment surrounding a person affects participation in daily occupations (AOTA, 2014), including sleep.

\section{Physical Factors Affecting Sleep}

The physical environment involves nonhuman surroundings and objects (AOTA, 2014), which can significantly affect sleep participation. In particular, the less control a person has over his or her environment, the more it negatively affects sleep (Green \& Nakopoulou, 2015). It is also important to consider that there is no ideal sleeping environment that suits every person (Green \& Nakopoulou, 2015), and individuals who evaluate sleep should examine how various environmental factors affect sleep on a case-by-case basis (Leland et al., 2014). The 
environmental factors that typically have the most significant effect on sleep are temperature, light, and noise (Green \& Nakopoulou, 2015).

The NSF (n.d.) suggests that temperatures above 75 degrees Fahrenheit and below 54 degree Fahrenheit will disrupt sleep. An ideal sleeping temperature ranges between 65 and 68 degrees Fahrenheit (Green \& Nakopoulou, 2015). In general, people tend to use clothing and bedding to maintain body temperatures and to create a favorable climate in bed (OkamotoMizuno \& Mizuno, 2012, as cited in Green \& Nakopoulou, 2015). Similarly, variations in lighting affect a person's sleep.

Light is an important regulator of the sleep-wake cycle, with darkness being the cue for production of melatonin in the evening (Green \& Nakopoulou, 2015). When a person is exposed to artificial lighting such as televisions and cell-phone lights before bedtime, there is a disruption in melatonin synthesis that can also activate arousal-promoting neurons (Green \& Nakopoulou, 2015). This information suggests that paying attention to lighting when preparing for and participating in sleep is very important (Green \& Nakopoulou, 2015). Additionally, noise levels can also affect sleep.

The World Health Organization recommends limiting background noise to no higher than 30 decibels (Berglund, Lindvall, \& Schewela, 1999), but noise has a variable effect on sleep depending on the person and the situation (Green \& Nakopoulou, 2015). Therefore, it is important to consider individual factors when determining sensitivity to noise (Green \& Nakopoulou, 2015). Sociocultural factors affecting sleep are also specific to each individual and should be considered when evaluating sleep. 


\section{Sociocultural Factors Affecting Sleep}

The cultural context involves the customs, beliefs, and activity patterns that influence a person's activity choices (AOTA, 2014), and various cultural attitudes can affect sleep participation. For example, various religions often acknowledge the benefits of good sleep habits and the routines related to sleep and meditation (Green \& Durant, 2015). There is also a specific culture of Western sleep, where there is an emphasis on solitary sleep, or sleep with only one co-sleeper (Green \& Durant, 2015). Additionally, Western cultures emphasize clear bedtimes that are often followed throughout life (Green \& Durant, 2015). Where a person sleeps can also be affected by culture, considering that not everyone sleeps on a mattress that sits off of the ground (Green \& Durant, 2015). Culture may also dictate the activities that occur in the bedroom (Green \& Durant, 2015). Similarly, sociological factors affect sleep.

The social environment includes the people or groups that affect a person's expectations, norms, and social routines (AOTA, 2014). In a sleeping environment, the effects of pair-sleep is an example of how social contexts can affect sleep participation. In some cultures, it is uncommon for couples and/or mother and child to sleep together, where these practices are common in other cultures (Green \& Durant, 2015). Gender roles can also affect sleep. For example, females are generally more receptive to the presence of others because of maternal instincts (Green \& Durant, 2015). Just as these factors are individually determined, temporal factors that affect sleep are also specific to the individual.

\section{Temporal Factors Affecting Sleep}

Temporal considerations include the stage, timing, duration, and rhythm of an activity (AOTA, 2014). The stage of a person's life, the time of the day that he or she participates in 
sleep, and the duration and rhythm of sleep can all affect sleep participation. In addition, how people use their time can affect their sleep habits (Green \& Durant, 2015).

There is a significant amount of research on the recommended amount of sleep for optimal functioning and on how certain activities that people participate in, particularly during the evening hours, affect sleep. Other considerations include how a person spends his or her time during the day and in regards to sleep patterns. For example, monophasic sleep is associated with increased productivity, where segmented sleep is often considered a waste of time in Western cultures (Green \& Durant, 2015). Daytime napping has also been linked to a reduction in effectiveness of nighttime sleep (Stepanski \& Wyatt, 2003), and having regular bedtimes and wake times improves sleep by supporting circadian rhythm cycles (Stepanski \& Wyatt, 2003). Additionally, the time of the year can affect sleep, as the onset of daylight and nighttime shifts with the changing of the seasons. Various changes in temporal and other contextual factors can either support sleep participation or create disruptions in sleep.

\section{Disruptions in Sleep}

Sleep disruptions often have negative effects on various aspects of a person's life, including functional participation, mental functioning, quality of life, and health. In fact, prolonged sleep problems can negatively affect a person's psychological and physical well-being and can increase adverse symptoms of health problems and health conditions (Wheaton et al., 2011).

\section{Functional Implications of Decreased Sleep}

Insufficient sleep is a multifaceted problem. For example, decreased sleep can negatively affect brain functioning, mood, and healing, and inadequate sleep has been linked to heart disease, stroke, diabetes, obesity, cancer, and even mortality (Tester \& Foss, 2018). 
Additionally, many health conditions negatively affect sleep quality, which can then lead to disruptions in other areas of life. O'Donoghue and McKay (2012) reported changes in functional participation, psychological and mental functioning, and personal relationships for individuals with obstructive sleep apnea, which is a common sleep illness where oxygen is prevented from reaching the lungs thus causing the affected person to awaken multiple times. Older adults, in general, are also at a higher risk for disturbed sleep due to higher incidences of chronic diseases and other age-related illness and conditions (Lorenz et al., 2014). In fact, the risk for disability in older adults can be reduced with improved sleep (Lorenz et al., 2014). Another population that is particularly at risk for increased disruptions in sleep habits involves individuals after BI (Duclos et al., 2015).

\section{Sleep after Brain Injury}

Mathias and Alvaro (2012) report that approximately half of adults after TBI have a sleep disturbance, but they also acknowledge that this prevalence varies due to variable diagnostic criteria, sleep measures, and population samples in numerous reports. After stroke, sleep disturbances occur in approximately $40 \%$ to $80 \%$ of cases (Suh, Choi-Kwon, \& Kim, 2014). These associated sleep problems will likely affect the recovery process and other symptoms associated with a BI, such as pain, cognitive deficits, fatigue, depression, and anxiety (KyungLim et al., 2017; Mathias \& Alvaro, 2012; Nakopoulou, Gaylarde, \& Green, 2015; Ouellet \& Morin, 2004). Circadian rhythm disorders are also common after TBI, which typically increases daytime sleepiness (Nakopoulou et al., 2015). Fatigue, insomnia, parasomnias, and sleep-related movement disorders are common after stroke (Raphaelson \& Inati, 2012). Additionally, there is evidence to suggest that the severity of the BI might be a factor in determining the type of sleep disturbance that is experienced after TBI (Bogdanov et al., 2017). 
For individuals after BI, sleep deprivation can be detrimental. It often causes changes in attention and memory, affects executive functioning and the learning of verbal and nonverbal information, and can lead to a decline in participation in ADL and other functional tasks (Duclos et al., 2015). Disruptions in sleep performance for individuals after BI have also been linked to depression and anxiety, reduced productivity in daily activities (Duclos et al., 2015), and decreased quality of life after TBI and stroke (Duclos et al., 2015; Suh et al., 2014). While there is limited evidence on the effectiveness of specific treatment options for sleep disturbances after $\mathrm{BI}$, non-pharmacological sleep interventions may be beneficial for this population and warrant further study (Nakopoulou et al., 2015).

\section{Sleep Hygiene and Behavioral Sleep Strategies}

Sleep hygiene is a list of behaviors or other sleep-related factors that are used to support improved sleep quality and quantity (Stepanski \& Wyatt, 2003). These behaviors can be used as stand-alone treatments or as part of a sleep hygiene program (Stepanski \& Wyatt, 2003). Sleep hygiene interventions are indicated for individuals who engage in habits or who are surrounded by environmental factors that do not support initiation and the ability to sustain good sleep (Posner \& Gehrman, 2011). Depending on the source, sleep hygiene can be categorized into several different groups of behaviors or modifications or behaviors of environmental factors.

\section{Sleep Hygiene Overview}

Green and Hicks (2015) identify sleep hygiene categories as factors related to the environment, food and drink, and behaviors, while Bogdanov et al. (2017) identify four domains of sleep hygiene. These domains include sleep homeostatic factors, circadian factors, medication and drug effects, and arousal in sleep setting (Bogdanov et al., 2017). Others identify sleep hygiene as simply a list of recommendations that encourage people to participate in behaviors 
that support good sleep and to avoid behaviors that interfere with normal sleep patterns (Stepanski \& Wyatt, 2003). In general, sleep hygiene practices and interventions are designed to support healthy sleep habits based on individual needs.

A major focus in developing healthy sleep habits involves modifications to the sleeping environment. As a general rule, the bedroom should always be conducive to sleep (Green \& Hicks, 2015; Posner \& Gehrman, 2011). Bedrooms should be kept cool, should have good ventilation, should be quiet and dark, and should be reserved for only sleep whenever possible (Green \& Hicks, 2015). Earplugs or the use of white noise can help to drown out other disruptive noises (Posner \& Gehrman, 2011). Other suggestions for the bedroom include removing electronic equipment and placing clocks in a place that cannot be visualized from the bed (Green \& Hicks, 2015), and to avoid co-sleeping with one's pets (Posner \& Gehrman, 2011). Another important consideration for sleep hygiene relates to the consumption of substances.

Caffeine and alcohol should be avoided in evening hours, as they can disturb sleep (Stepanski \& Wyatt, 2003). While alcohol can decrease the time required to fall asleep, it often results in fragmented sleep later in the night (Green \& Hicks, 2015; Stepanski \& Wyatt, 2003). However, having a glass of wine with dinner or as a part of the evening routine to wind-down may not be disruptive to sleep (Green \& Hicks, 2015). The general rule for consumption of caffeine is that it should be avoided at least 6 hours before sleep (Green \& Hicks, 2015), and liquids, in general, should be restricted in the last 4 hours before bedtime (Posner \& Gehrman, 2011). Nicotine should be avoided close to bedtime as well, and it is best to avoid going to bed on a full stomach or while feeling hungry (Green \& Hicks, 2015). Therefore, a light snack that includes carbohydrates may be beneficial about 1 hour before bedtime (Posner \& Gehrman, 2011). A significant consideration for sleep hygiene involves personal behaviors. 
Many behavioral routines and modifications are often required to achieve good sleep habits and sleep hygiene. For example, Mairs and Mullan (2015) discuss behavior change techniques that can be used during interventions to support improved health behaviors. This includes teaching individuals with sleep deficits to practice self-regulation and self-monitoring through practices such as using sleep diaries, making the sleep environment restful, and limiting going to bed feeling hungry or thirsty (Mairs \& Mullan, 2015). The most successful behavior change techniques involve strategies to promote problem solving and planning to achieve established goals (Mairs \& Mullan, 2015).

Other behavioral strategies to improve sleep hygiene are as simple as having regular bedtimes and wake times and minimizing daytime napping (Green \& Hicks, 2015; Posner \& Gehrman, 2011; Stepanski \& Wyatt, 2003). Exercise can help to deepen sleep and provide more restorative sleep, but should be done in the late afternoon or early evening (Posner \& Gehrman, 2011). Participating in a winding down routine can also be helpful. This might include switching off electronics before bedtime, taking a hot bath, and practicing relaxation techniques (Green \& Hicks, 2015). Additionally, sleep restriction therapy (SRT), or sleep scheduling, can also be beneficial for some people with sleep deficits.

\section{Sleep Restriction Therapy}

The goal of SRT is to restrict the amount of time in bed to match the time that the person normally sleeps, even in instances where the person has recently had a bad night's sleep (Green \& Hicks, 2015). For SRT to be effective, the focus is on initially increasing sleep efficiency and then slowly increasing the time spent in bed once efficiency is improved to approximately $90 \%$, meaning that $90 \%$ of the time spent in bed was during actual sleep (Green \& Hicks, 2015). During SRT, it is important to follow selected sleep hours and times and to keep a record of 
sleep performance (Green \& Hicks, 2015). SRT should be used cautiously, as it is not always the best approach for every person experiencing sleep deficits (Green \& Hicks, 2015). An approach that can be very effective for individuals with all types of sleep deficits is stimulus control therapy (SCT; Bootzin \& Perlis, 2011).

\section{Stimulus Control Therapy}

SCT is useful for individuals who have sleep onset problems (Bootzin \& Perlis, 2011). SCT involves learning to associate the bed and bedroom with sleep and to reduce cues that associate the bed and bedroom with arousal. There are several basic instructions related to SCT. SCT includes lying down and going to bed only when feeling sleepy in order to help a person to become more aware of the body's cues related to sleepiness (Bootzin \& Perlis, 2011). The bed should not be used for anything other than sleep in order to associate the bed and bedroom with falling asleep. The only exception to this rule is sexual activity (Bootzin \& Perlis, 2011). Additionally, if a person is unable to fall asleep, he or she should get up and go to another room to limit the amount of time of being awake while in bed (Bootzin \& Perlis, 2011). SCT also involves setting an alarm clock to get up at the same time each morning, regardless of how much sleep the person got during the night. This helps to set a consistent sleep rhythm (Bootzin \& Perlis, 2011). Finally, individuals following SCT are instructed to avoid napping during the day so that any sleep deprivation from the previous night will help to facilitate sleep the next night (Bootzin \& Perlis, 2011). There are modifications and considerations to be made when individuals have physical limitations affecting their safety or ability to get out of bed and if there is limited cognitive capacity (Bootzin \& Perlis, 2011). SCT and SRT are often utilized as part of cognitive-behavioral therapy for insomnia (CBT-I), which is a common intervention for people with insomnia. 


\section{Cognitive-Behavioral Therapy for Insomnia}

CBT-I is a treatment intended to promote increased awareness of factors that affect sleep and to teach methods to either manage or change these behaviors (Tester \& Foss, 2018), and it must be delivered by a trained professional. CBT-I is used to decrease insomnia, alleviate dysfunctional thoughts, and reduce emotional stress that leads to disruptions in sleep, and it has been shown to help reduce sleep disruptions (Ouellet \& Morin, 2004). It can be used in conjunction with other sleep interventions as well (Tester \& Foss, 2018).

\section{Effectiveness and Implications of Sleep Hygiene}

Sleep hygiene can have a positive effect on various populations (Bogdanov et al., 2017;

Green \& Hicks, 2015; Stepanski \& Wyatt, 2003) and has been demonstrated to be an important predictor of sleep quality for individuals across the lifespan (Bogdanov et al., 2017). Having better knowledge of sleep hygiene has also been liked to better sleep practices (Bogdanov et al., 2017), which improves sleep participation. It is also important to consider that for sleep hygiene to be effective, it is necessary to have replication and monitoring of sleep hygiene practices to ensure consistency (Mairs \& Mullan, 2015). Additionally, sleep hygiene education and interventions should be presented in a reasonable format, such as using paper and pencil instruments that are quantifiable and yield information supporting intervention approaches that can be tailored to each client's needs (Bootzin \& Perlis, 2011). To effectively evaluate a client's sleep participation, appropriate sleep assessments should be utilized.

\section{Measures of Sleep}

When a client acknowledges having sleep problems, it is important to identify factors related to the client's sleep habits and sleep participation. There is a variety of commonly used sleep assessments that can be utilized by health care professionals to further evaluate sleep and to 
measure outcomes related to sleep participation (Green \& Hicks, 2015). Assessments can either be objective or subjective.

\section{Objective Sleep Measures}

To diagnose sleep disorders, polysomnography is considered the "gold standard" because it assesses sleep physiology (Meltzer, Hiruma, Avis, Montgomery-Downs, \& Valentin, 2015) using electrodes to measure brain waves and other physiological responses during sleep. However, polysomnography is costly, is administered in a sleep clinic or sleep laboratory, and does not provide information regarding sleep patterns over multiple nights (Meltzer et al., 2015). To measure sleep over multiple nights, actigraphy is often utilized (Kang et al., 2017).

Actigraphs are portable devices worn on the wrist that use accelerometers to detect physical movements. These movements indicate if a person is asleep or awake (Meltzer et al., 2015) and measure total sleep time. However, actigraphy can also be expensive and requires specialized software and training to interpret data (Kang et al., 2017). There are several nonwearable trackers, such as devices embedded in a mattress, bed sheets, pillows, or that sit on a bedside table, that track light, movement, heart rate, and temperature to estimate sleep quality (Liu, Ploderer, \& Hoang, 2015). While these devices do not have to be worn on the body, there is minimal evidence on the effectiveness of such devices (Liu et al., 2015). Additionally, due to variability of sleep environments from person to person, due to the portability and sensitivity of various trackers (Liu et al., 2015), and to maximize cost-effectiveness in a study, commercial sleep trackers are often a more feasible option.

There is an abundance of commercial sleep trackers available to consumers, and such devices have become very popular among individuals with and without sleep disorders (Kang et al., 2017; Meltzer et al., 2015). Commercial sleep trackers are user friendly, provide immediate 
information about sleep by synchronizing to smart phones, and are affordable and attractive devices (Kang et al., 2017). Additionally, they are a more objective measure of sleep than selfreported tools (Liu, Wong, Zwetsloot, Hsu, \& Tsui, 2019). For example, wearable devices such as Fitbit trackers and Jawbone trackers and commonly used devices.

The Fitbit Flex is one of the most popular commercial sleep trackers (Kang et al., 2017) with several studies documenting the utility of this device in regards to sleep tracking (Cook, Eftekari, Dallmann, Sippy, \& Plante 2017; Kang et al., 2017; Morhardt, Luckenbaugh, Goldstein, \& Faerber, 2017). This device was considered for the current study because of the slim design and because it does not have a large watch face that allows for adjustment of settings. This was preferable in the current study so that participants could not interfere with device settings on the device itself, which could potentially affect the reliability of the data obtained from the Fitbit device. However, the Fitbit Flex does not measure heart rate, which is an important indicator in estimating the stages of sleep, restlessness, and periods of wakefulness (Kosecki, 2017). Therefore, this device was eliminated from consideration for the current study. Additionally, the Fitbit Alta HR was originally selected for the current study due to its ability to track heart variability and its slim design, but this device was retired prior to initiation of the study. The Fitbit Inspire HR is operationally and physically similar to the Fitbit Alta HR, making it a viable substitute. To supplement data from objective sleep measures, subjective measures of sleep are very important and offer a more individualistic understanding of a person's sleep. 


\section{Subjective Sleep Measures}

Sleep is a very subjective experience for individuals, and having measures of sleep that evaluate the subjective aspects of sleep is crucial to understanding a person's experiences with sleep deficits. Subjective measures are in the form of assessment tools or sleep diaries.

Subjective assessment tools. The Pittsburg Sleep Quality Index (PSQI) is a widely used tool to measure various aspects of sleep participation by evaluating sleep quality and the frequency and severity of sleep-related problems (Buysse, Reynolds, Monk, Berman, Kupfer, 1989). The PSQI is a valid and reliable self-report questionnaire (Mairs \& Mullan, 2015), but because items are rated over the previous month, the PSQI was not the best measure of sleep for the current study. The Occupational Impact of Sleep Questionnaire is commonly used to evaluate how work is affected by sleep quality and examines difficulties in meeting work demands in the past week (Green \& Hicks, 2015), which was not being measured in the current study. The Glasgow Sleep Effort Scale (Broomsfield \& Espie, 2005) assesses sleep effort and anxiety regarding sleep, which are items that were also not being measured in the current study. The Medical Outcomes Study (MOS) Sleep Scale measures multiple dimensions of sleep, can be utilized with a variety of populations, and is quick to administer and score, but it does not give a cutoff score and has no established validity (Smith \& Wegener, 2003), making it less favorable to use in the current study. In additional to established subjective assessment tools, an established sleep diary was also utilized in the current study.

Sleep diaries. The sleep diary is considered the "gold standard" for measuring subjective sleep (Carney et al., 2012). There are several widely used sleep diaries available in research and in practice, but there is a lack of a standardized format for sleep diaries thus far (Carney et al., 2012). The Pittsburg Sleep Diary (PSD) is a popular sleep diary that gives a subjective report of 
sleep and wake behaviors and is used to quantify sleep using two questionnaires, which are completed after waking and before going to bed (Monk et al., 1994). Both questionnaires contain items related to various habits and factors that could affect sleep participation and sleep duration (Monk et al., 1994). The NSF also has a printable sleep diary available on the NSF website. This diary has two forms, which are also completed in the morning and at the end of the day (NSF, n.d.). However, because both the PSD and the NSF sleep diary are completed in the morning and at bedtime, a shorter and simpler version was desired for the current study with the BI population. Information obtained from a sleep diary, other subjective measures, and objective measures of sleep can be utilized by occupational therapists to evaluate sleep participation and to make recommendations for appropriate sleep interventions in occupational therapy practice and research.

\section{Occupational Therapy and Sleep}

While sleep is currently gaining recognition in the fields of occupational therapy and occupational science, sleep has not always been present in occupational therapy research or clinical practice. In fact, sleep has often been under-addressed by occupational therapists in health care, research, and academia. However, there is some evidence to suggest that the significance of sleep was acknowledged in the early years of occupational therapy.

\section{Historical Overview}

Adolf Meyer was a pioneer in the field of occupational therapy, recognizing the need for a balance between work, play, rest, and sleep to promote health (Meyer, 1922). While Meyer (1922) emphasized the importance of balance, he argued that achieving balance occurred by actual doing. In the time of institutional care, attention was not spent on the lack of opportunity for sleep, because nurses were expected to take care of a patient's need for sleep (Green, 2015). 
Additionally, the emphasis in therapy was on work and activity, and sleep was not likely to be a part of occupational therapy's role in these early years (Green, 2015). In fact, occupational therapy models and standard occupational therapy textbooks have historically overlooked sleep (Green, 2015). Sleep is often not included in the occupational therapy curricula at educational institutions as well (Tester \& Foss, 2018), which leaves many occupational therapists unaware of the potential role of occupational therapy in the promotion of good sleep hygiene and healthy sleep habits.

Not surprisingly, Fung et al. (2013) report that occupational therapists are not routinely assessing sleep or even recognizing when sleep is an issue for their clients. Fortunately, AOTA (2008) reclassified rest and sleep as areas of occupation in the OTPF. AOTA (2012) also acknowledges that adequate sleep supports optimal occupational performance and engagement in life activities, thus supporting occupational therapy's role with sleep.

\section{Occupational Therapy's Role with Sleep}

The process of delivering occupational therapy services involves evaluation, intervention and targeting outcomes (AOTA, 2014). During an evaluation, the occupational therapist completes an occupational profile to evaluate the client's occupational history, interests and needs, and patterns of occupational performance (AOTA, 2014). This process includes the identification of factors that either support or hinder performance (AOTA, 2014). Performance skills, client factors, and contextual factors are also considered (AOTA, 2014). Given that occupational therapists have the skills necessary to evaluate various aspects of occupational performance and personal and environmental factors that could be affecting sleep participation, they are more than capable of addressing sleep and sleep problems with clients in a variety of settings (Fung et al., 2013). In fact, occupational therapists are often more aware of how various 
disruptions in daily routines can affect functional and meaningful activities than other health professionals, based on occupational therapy's scope of practice. This makes occupational therapists uniquely qualified to evaluate clients' sleep habits and to offer suggestions for interventions to improve sleep participation.

During the intervention phase of the occupational therapy process, an intervention plan is developed in collaboration with clients based on selected theories and evidence (AOTA, 2014). Interventions that support improved client performance and participation are implemented and are directed at identified outcomes (AOTA, 2014), which would involve non-pharmacological sleep interventions to address sleep problems and to support improved sleep participation for the current study.

To address sleep problems individually, or as part of a sleep program, occupational therapists can recommend environmental modifications, changing personal habits and behaviors, and better time and stress management in order to enhance sleep participation (Fung et al., 2013). Occupational therapists can also work with clients to modify their sleep performance patterns by following sleep schedules and reducing nighttime stimuli (Leland et al., 2016). Contextual and environmental factors should also be evaluated by an occupational therapist, and modifications should be suggested as needed, such as adjusting temperatures and nightly routines and minimizing environmental stimuli with the use of eye masks, music, or headphones (Leland et al., 2016). Assisting clients in keeping sleep diaries, offering sleep hygiene education, encouraging deep breathing and relaxation techniques, and suggesting anxiety management strategies are other potential roles for occupational therapy (Gentry \& Loveland, 2013). Each of these interventions can be implemented by occupational therapists in any setting and should be part of an interdisciplinary approach to addressing sleep. Occupational therapists should 
network with other healthcare professionals to address sleep problems as well (Tester \& Foss, 2018) and can recommend further evaluation by a sleep specialist if needed.

The suggested interventions require minimal clinical effort from occupational therapists (Tester \& Foss, 2018) and would be easy to incorporate into typical occupational therapy sessions, as these types of interventions are already addressed by occupational therapists in relationship to other areas of occupational performance. Additionally, by incorporating sleep assessments and sleep interventions into everyday occupational therapy practice, the potential benefits for the clients are more than just improved sleep. Addressing sleep problems can lead to increased functional participation, improved health and well-being, and an overall better quality of life for clients. These outcomes would be evaluated during the occupational therapy process, and information obtained from the outcome assessments would be used to plan future actions with clients (AOTA, 2014). Several studies have examined the potential role for occupational therapists in regards to the promotion of healthy sleep habits, but outcome measures for occupational therapy-directed sleep interventions are lacking in the occupational therapy literature (Smallfield \& Lucas Molitor, 2018; Tester \& Foster, 2018). However, occupational therapists are well positioned to introduce non-pharmacological sleep interventions for clients in a variety of clinical settings.

\section{Non-pharmacological Sleep Interventions}

Historically, pharmacological interventions have been the main treatment for individuals with sleep disturbances (Wang et al., 2016). However, sleep medications often have adverse effects, such as medication tolerance and/or dependence (Bogdanov et al., 2017; Wang et al., 2016), and risk of confusion and falls (Wang et al., 2016). As an alternative to medications, nonpharmacological interventions for sleep are safer (Neuendorf et al., 2015; Wang et al., 2016) and 
often more cost-effective options (Neuendorf et al., 2015). There are many studies across multiple disciplines that examine the effectiveness of non-pharmacological sleep interventions, which often target modifications to personal, environmental, and occupational factors.

\section{Person-based Interventions}

Interventions that are person based aim to change client factors, including body functions and body structures that affect performance (AOTA, 2014). Person-based interventions might include sleep restriction training, which includes setting an alarm to wake up at the same time every day (Green \& Hicks, 2015). Relaxation therapy, such as meditation and deep breathing activities, can also be helpful as a person-based or occupation-based interventions, as demonstrated by Vitiello, Rybarczyk, Von Korff, and Stepanski (2009) in a study that yielded improvements in sleep outcomes for adults following relaxation therapy. Person-based interventions may also behavioral in nature.

\section{Environment-based Interventions}

The environment includes "the external physical and social conditions that surround the client and in which the person's daily life occupations occur" (AOTA, 2014, p. S28). Interventions that target environmental factors involve modifications to various aspects of the environment that inhibit sleep participation. Examples of environmental modifications might include the use of sleep-inducing music and a sleep mask to be worn at bedtime. These interventions have been shown to be effective interventions for individuals in the hospital setting (Ryu, Park, \& Park, 2012) and in various other environments.

\section{Occupation-based Interventions}

Occupation-based interventions involve modifications to occupations, which are the life activities that a person engages in (AOTA, 2014). An example of occupation-based 
interventions might include avoiding daytime naps, instead choosing to participate in a meaningful occupation during available daytime hours. Individuals might also participate in light physical activities, such as stretching, walking, and yoga, in either the morning or the afternoon. These activities have been shown to be effective in improving neuropsychological performance and subjective sleep for older adults (Benloucif et al., 2004). Additionally, stimulus control, which involves limiting the bedroom to sleep and sexual activity, involves potential changes to occupational patterns. Many sleep interventions address personal, environmental, and occupational factors, which are also addressed in several established theoretical frameworks that can be used to guide the development and implementation of the proposed study.

\section{Guiding Theoretical Frameworks}

Several theoretical perspectives and models can be applied to a study examining the effectiveness of occupational therapy-guided sleep interventions for individuals after BI who are participating in a community re-entry day treatment program. Many theoretical models depict how various aspects of the person and the environment interact to affect performance and occupational engagement, such as the PEO model, OA, and the social-cognitive theory. While the PEO model and OA are specific to occupational therapy practice and research, the socialcognitive theory offers a multi-disciplinary perspective for understanding sleep and factors related to sleep performance. Utilizing these three models can help to guide the development and implementation of interventions that will suggest adjusting various aspects of the person, the environment, and occupations to improve occupational performance, or sleep participation for purposes of the proposed study. In fact, the PEO model was used to frame the design of the study's sleep interventions due to its explicit emphasis on the person, environment, occupation, and occupational performance. 


\section{Person-Environment-Occupation Model}

For the dissertation study, a goal was to determine whether interventions based on person factors, environmental factors, occupation factors, or a combination of these factors, are effective for individuals participating in a community re-entry day treatment program after BI. The expectation was that, by implementing interventions based on these key elements, participants would have improved sleep participation. The basic premise of the PEO model is to examine the dynamic transaction between the person, the environment, and occupation and to understand how this interaction affects occupational performance (Law et al., 1996). The achieved sleep participation in this study related to what the PEO model describes as occupational performance (see Figures 1 and 2).

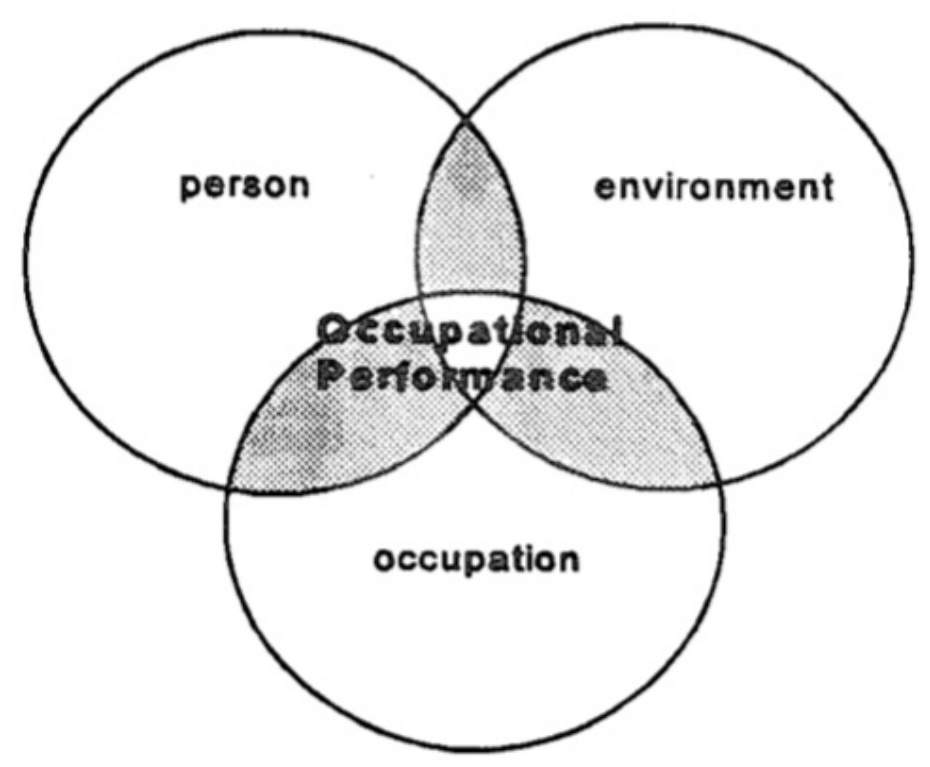

Figure 1. The person-environment-occupation model of occupational performance (Law et al., 1996, p. 19). 


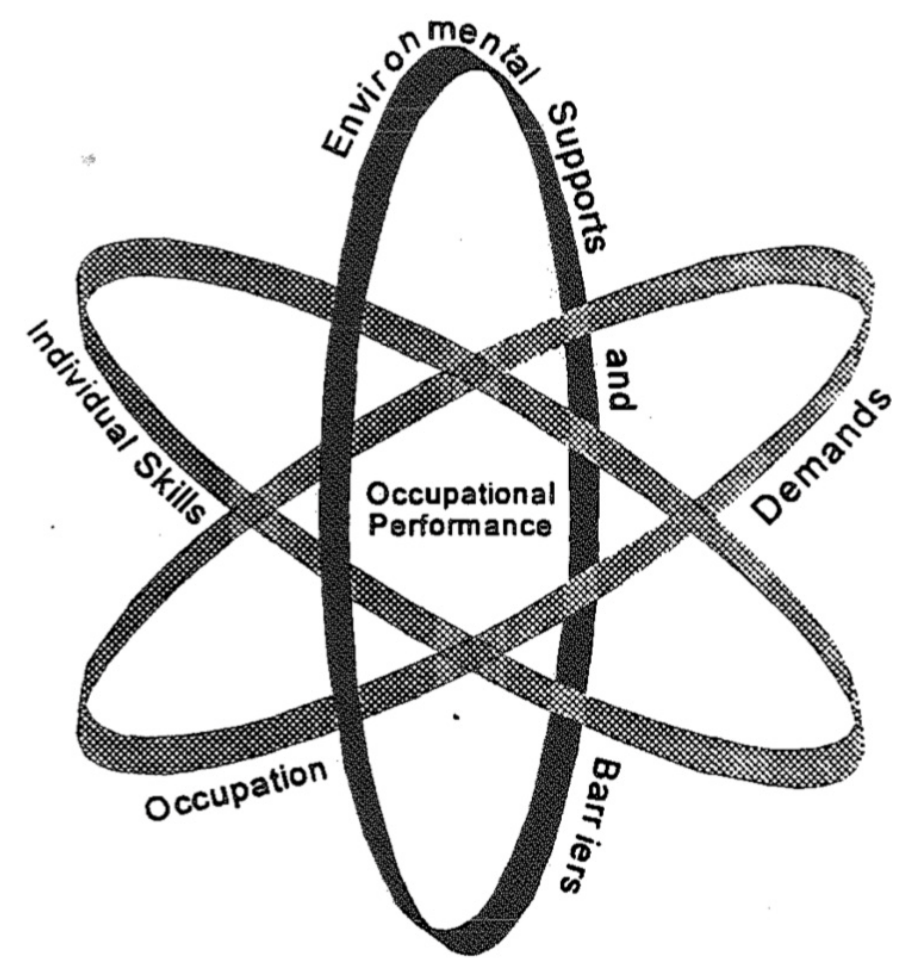

Figure 2. Detailed person-environment-occupation model of occupational performance (Law et al., 1996, p. 15).

Based on the assumptions of the PEO model, the greater the fit between the three elements, the greater the sleep participation will be. PEO also considers clients' interests and needs to be the most important aspects for consideration, and through participation in meaningful occupations, a person's need for fulfillment, personal expression, and self-maintenance within his or her roles and within the environment are enhanced (Law et al., 1996).

Because the PEO model values the interests and needs of clients, interventions for the dissertation study were selected based on the specific needs of the BI population. Additionally, the PEO model highlights the person as a holistic being with a unique set of attributes, skills, and a variety of roles (Law et al., 1996). By conducting pre-screening assessments, the principal investigator had a more holistic understanding of the clients and the factors that either supported or inhibited clients' sleep participation. 
Occupational therapy evaluations should assess aspects of the person, the environment, and the occupation. Occupational therapy interventions should target these three elements in various ways, and the outcome of intervention is evaluated by measuring occupational performance (Law et al., 1996). Interventions in the study focused on various aspects of the person, the environment, and the occupation, and outcomes of the study were evaluated by objectively and subjectively measuring sleep participation. While the PEO model evaluates the interaction between the elements of person, environment, and occupation, OA looks at how people use occupation as a means for adaptation. This model can be used to explain how participants adapted to changes within themselves and within the environment to support improved sleep participation after BI.

\section{Occupational Adaptation}

Under the principles of OA, occupation is a means by which people adapt to change, and the desire to participate in occupations leads to adaptation (Jackson \& Schkade, 2001). For the context of the dissertation study, occupational adaptation occurred when participants overcame personal and environmental barriers to achieve improved sleep participation (see Figure 3). The process of occupational adaptation promotes increased occupational functioning and relative mastery in occupational activities (Jackson \& Schkade, 2001). Additionally, a person's desire for mastery combined with environment stimuli calls for an occupational response and a demand for mastery, and a person's response to an occupational challenge also promotes relative mastery (Schkade \& Schultz, 1992).

In the dissertation study, various interventions based on personal, environmental, and occupational factors were used as a means to promote the desired occupational response of 
improved sleep. Sleep is an occupation, and improved sleep participation was the desired end result, thus promoting improved occupational engagement.

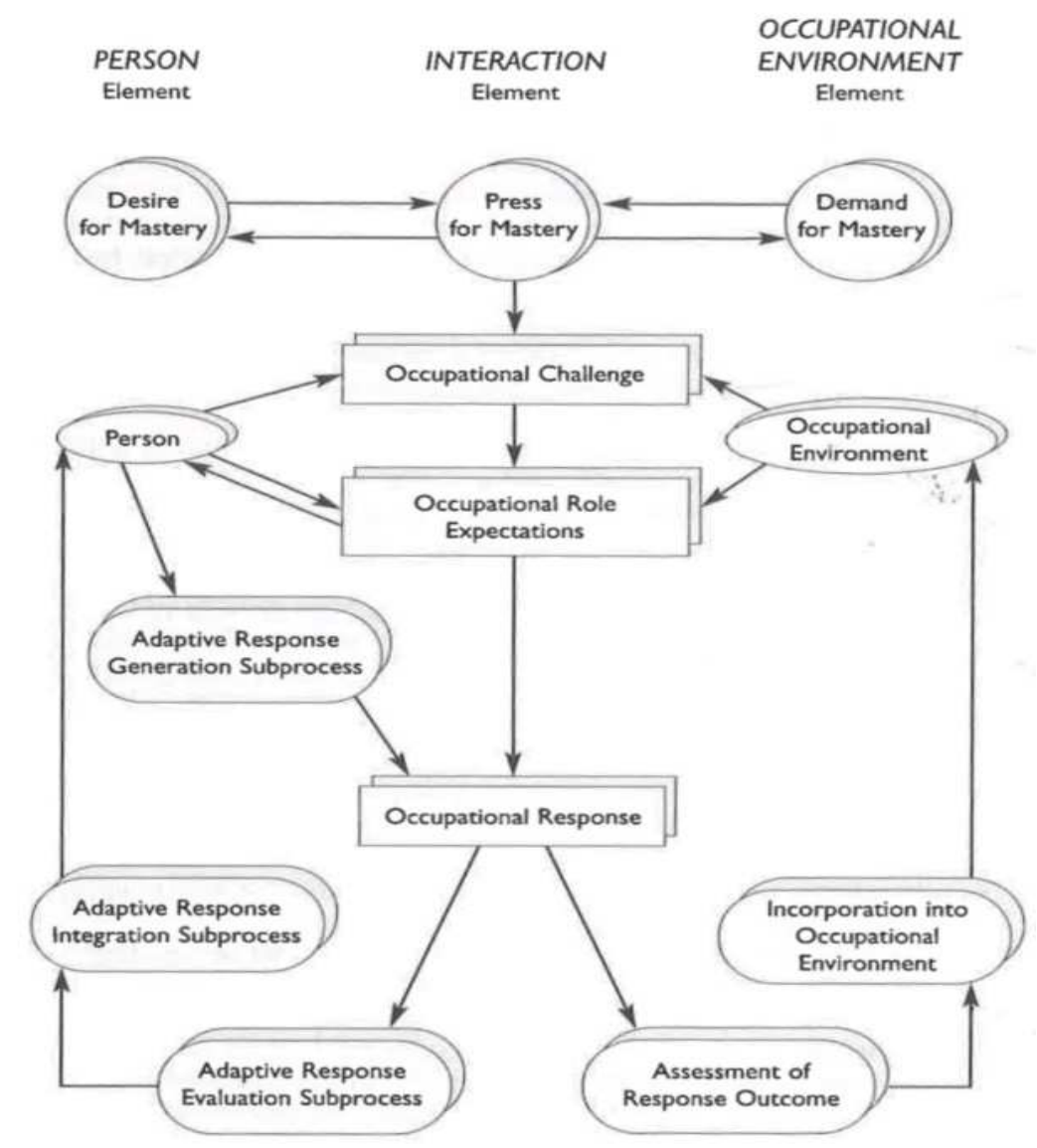

Figure 3. The occupational adaptation process (Schkade \& McClung, 2001, p. 5).

After a person experiences a BI, there are obvious changes within the person system, which involves the individual's sensorimotor, cognitive, and psychosocial functioning. This is consistent with Schkade and Shultz's (1992) depiction of person systems. Additionally, when the client's living environment is also changed after BI, there are often extra challenges in regards to regaining and maintaining typical sleep patterns due to potential contextual barriers (e.g., variations in lighting, temperature, and noise).

These changes within the person and the environment systems create an occupational challenge, often making it difficult for the person to participate in adequate sleep. However, the 
participants' desire for improved sleep participation created his or her desire for mastery, with various environmental factors creating a demand for mastery. When participants decided to actively engage in the various sleep interventions, there was an adaptation gestalt, and when participants achieved improved sleep participation, relative mastery occurred. The researcher, who is an occupational therapist, was considered an environmental influence that helped participants to achieve internal adaptation by overcoming barriers to sleep participation. Based on the principles of OA, there was equal importance placed on sleep interventions that addressed person factors, environmental factors, and occupation-based factors. Additionally, some interventions focused on combinations of these three elements to determine which factors promoted occupational engagement with improved sleep participation. The social-cognitive theory also provided a framework for evaluating the dynamic process involving multiple factors and was used to understand how participants changed particular behaviors related to their health (National Cancer Institute, 2005).

\section{Social-Cognitive Theory}

Similar to the PEO model, the social-cognitive theory examines a dynamic process involving the interaction of personal factors, environmental factors, and human behavior (National Cancer Institute, 2005), as shown in Figure 4. Prior to conducting the dissertation study, the researcher evaluated the clients' personal factors in regards to their skills and abilities, determined which environmental factors acted as supports or barriers, and viewed how the clients' behaviors supported or inhibited changes in their sleep habits. The interaction between these elements represented reciprocal determinism, indicating that each factor influenced the others (National Cancer Institute, 2005). Based on principles of SCT, important considerations 
for person factors involved behavioral capacity, expectations, and self-efficacy (National Cancer Institute, 2005).

\section{Environmental Factors \\ - Social norms \\ - Access in community \\ - Influence on others (ability to change own environment)}

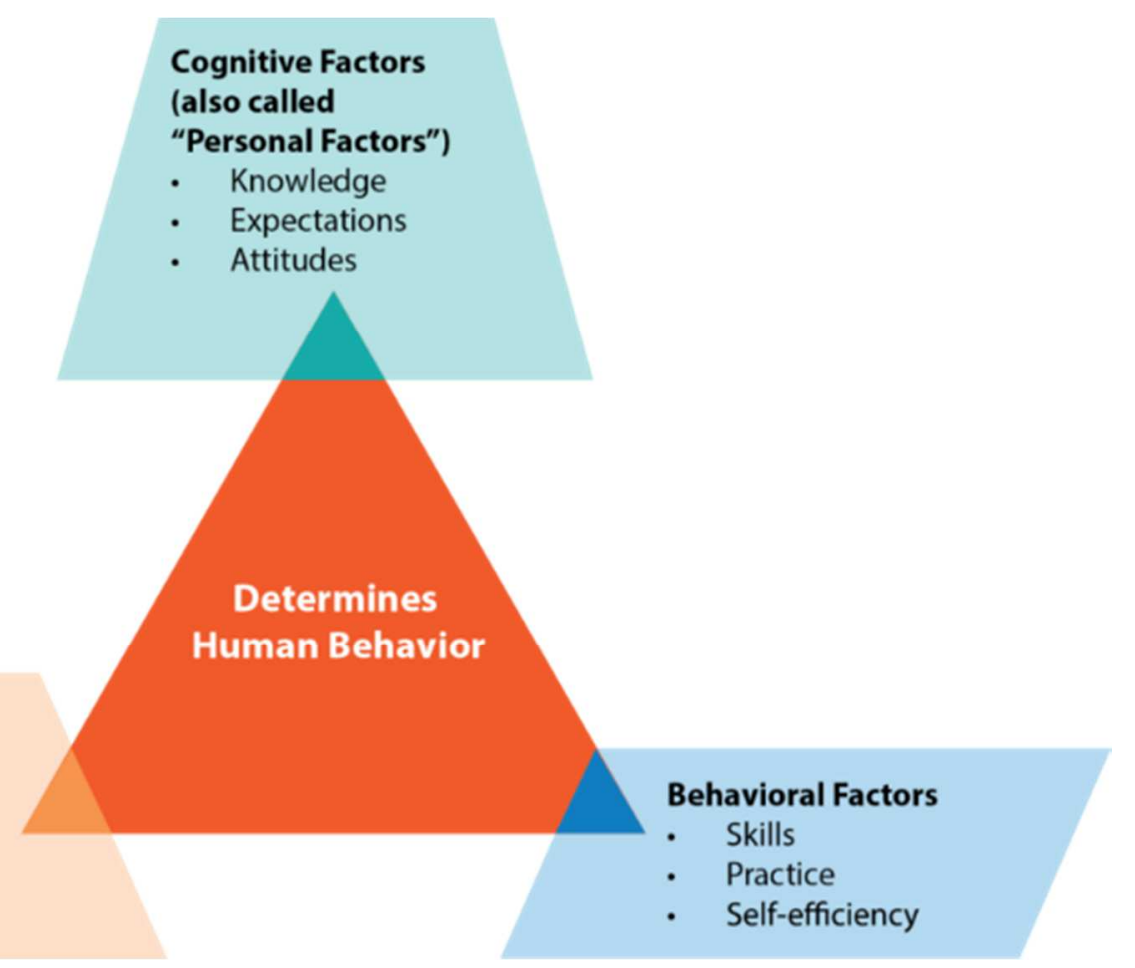

Figure 4. Social-cognitive theory. From "Social Cognitive Learning Theory," by Johns Hopkins University, 2016, https://sbccimplementationkits.org/sbcc-in-emergencies/social-cognitivelearning-theory/.

Behavioral capacity refers to a person's understanding and ability to perform a behavior (National Cancer Institute, 2005). Expectations are the person's anticipated results from taking action, and self-efficacy is the "confidence's in one's abilities to take action and overcome barriers" (National Cancer Institute, 2005, p. 20). For the dissertation study, participants' behavioral capacity and self-efficacy improved when the researcher and participating facility staff provided adequate education, instructions, and support with repeated review of techniques prior to the implementation of sleep interventions in the study. Specific interventions were also repeated daily until stability was achieved, which will also improved participants' self-efficacy. 
The expectations of implementing the sleep interventions were anticipated improvements in sleep participation.

Social-cognitive theory defines reinforcements as the responses to a behavior that indicate the likelihood that the behavior will be repeated (National Cancer Institute, 2005). Improvements in sleep participation served as a positive reinforcement, encouraging participants to continue to perform the sleep interventions. Additionally, people are able to learn from the actions of other people through observational learning or modeling (National Cancer Institute, 2005), which occurred when the researcher and facility staff modeled appropriate techniques for participants prior to implementing each intervention phase. Specific sleep interventions were selected based on the results of previous sleep intervention studies and to help support improved sleep habits and sleep participation for participants in the study.

\section{Summary of Applicable Theories}

The PEO model, OA, and the social-cognitive theory all served as guiding theoretical perspectives for the dissertation study due to their focus on the interaction between factors related to the person, the environment, and occupations or behaviors. Each of these perspectives looks at how multiple factors affect a person's ability to achieve a desired functional outcome, which parallels with the dissertation study's goal of promoting increased sleep participation for individuals after BI. By utilizing the principles and constructs identified by each of these perspectives, the dissertation study offered a holistic and inclusive approach to improving occupational performance for participants based on sound and evidence-based theoretical concepts. Additionally, several existing studies highlight the effectiveness of nonpharmacological sleep interventions using various designs and methodologies. 


\section{Review of Existing Studies}

Many previously conducted studies were used as a guide and to justify the selected methods and design of the current study. While many studies model successful designs or methodologies that support the study's methods, other studies present challenges that warrant further study and modifications to create successful research studies.

\section{Previous Successful Designs}

Several previous studies exemplify the implementation of successful designs that yield positive results and demonstrate the expected outcomes after an intervention period. The current study was an embedded mixed methods study utilizing a single-subject research design (SSRD) to fill the gaps left by previous studies in a thorough examination of the effectiveness of various sleep interventions while also attempting to understand the occupational implications of decreased sleep for this population. The qualitative strand is embedded in a larger study with a primary quantitative focus, where each strand of the study occurs in a sequential order.

Mixed methods designs. The effectiveness of using a mixed methods design with sequential data collection and analysis has been demonstrated in several previous studies (Lenz \& Lancaster, 2017; Nørgaard, Pedersen, \& Bjerrum, 2018; Van Bogaert et al., 2017). For example, Lenz and Lancaster (2017), Nørgaard et al. (2018), and Van Bogaert et al. (2017) effectively used both quantitative and qualitative measures to demonstrate positive intervention effects while also capturing the unique experiences of clients. Additionally, results from the qualitative analyses were able to validate the initial quantitative results in each of the studies. However, even though each of these studies highlights the strengths of using mixed methods research design with sequential data collection and analysis, there was a trend in limitations in these studies in that the outcome measures utilized were mostly self-report, subjective 
assessments. Additionally, Lenz and Lancaster suggested the use of single-case research designs, which were not utilized in any of the previously mentioned studies, to demonstrate positive treatment effects.

Single-subject research designs. The SSRD, also known as a single-case experimental design, has also been positively represented in the literature (Cosbey \& Muldoon, 2017; Desjardins, 2018; Ouellet \& Morin, 2007). Cosbey and Muldoon (2017) and Desjardins (2016) conducted quantitative single-case experimental design studies that were replicated across multiple participants. Both studies demonstrated overall effective research designs with positive intervention effects, thus increasing the potential to make a causal determination between study variables. With participants serving as their own controls in SSRD studies, researchers are able to measure responses to interventions at the individual level (Desjardins, 2016). However, both of these studies acknowledge the limitations of a SSRD in that generalizability is limited. While the benefits of using a SSRD are highlighted in these previously mentioned studies, other SSRD studies could benefit from improvement.

\section{Designs and Methodologies Needing Improvement}

The previously mentioned studies demonstrated overall positive effects in study design or methodology. However, some other studies have significant limitations in the study design or in the methodologies that make replication of these studies implausible (Gutman et al., 2017; Ouellet \& Morin, 2004; Walter, Hale, \& Smith, 2015; Wang et al., 2016).

Single-subject research designs. Walter et al. (2015) conducted a SSRD study of five participants in a two-phase study that did not yield positive treatment effects, potentially due to decreased appropriateness of the self-report outcomes measures. This highlights the importance of selecting relevant and sensitive assessment tools, including the use of both subjective and 
objective measures, which did occur in Ouellet and Morin's (2004) SSRD study. However, this study, which specifically evaluated sleep outcomes for individuals after TBI, also had several limitations.

While this quantitative study presented encouraging results supporting the effectiveness of non-pharmacological sleep interventions for individuals after TBI, the study included only one client and utilized a sleep lab for polysomnography data, which would be a challenging objective measure to utilize for many researchers. Additionally, sleep interventions often require strict adherence for successful outcomes (Ouellet \& Morin, 2004), which can be challenging to inforce in an outpatient setting. Other studies also evaluated sleep interventions for community-dwelling individuals using randomized controlled trials (RCT).

Randomized controlled trials studies. A study conducted by Wang et al. (2016) used a RCT to examine the effects of music intervention on sleep quality, but it demonstrated variable results and only utilized one subjective self-report questionnaire to evaluate outcomes. Objective measures of sleep quality may have yielded more effective results, which was demonstrated in another RCT conducted by occupational therapists to determine the effectiveness of three sleep interventions for healthy individuals (Gutman et al., 2017). While the study's selection of outcome measures, which included the use of self-report questionnaires, a sleep journal, and wrist actigraphy, was comprehensive, baseline and post-intervention outcomes were variable (Gutman et al., 2017). Additionally, the particular actigraph accelerometer that was used in the study only had moderate reliability (Gutman et al., 2017), which suggests the need for reliable objective measures. Finally, results of studies using healthy individuals cannot be generalized to people with acute or chronic health conditions (Gutman et al., 2017). Even though these studies specifically evaluated sleep outcomes, and the study by Gutman et al. (2017) demonstrates the 
potential for occupational therapists to address sleep problems, the methodology of these studies could use some improvements to create a stronger study.

\section{Designs and Methodologies Supporting the Study}

Many previously conducted studies highlight the benefits of non-pharmacological sleep interventions and provide good support for the design and methodology for the proposed study (Lillehei, Halcon, Savik, \& Reis, 2015; Martin, Marler, Harker, Josephson, \& Alessi, 2007; Ouellet \& Morin, 2007; Tse \& Hall, 2008.) Even though these studies are not occupational therapy specific, they could easily be replicated with modifications to support occupational therapy's role in addressing sleep problems for various populations.

For example, Martin et al. (2007) demonstrated that multicomponent nonpharmacological interventions significantly affect rest and activity cycles through the use of objective sleep measures in a quantitative study, and Lillehei et al. (2015) were able to subjectively and objectively measure sleep quantity and sleep quality in a quantitative RCT. Both studies show promising results for the effectiveness of sleep interventions, but Lillehei et al. acknowledge the limitations of self-reporting measures and the importance of seeking a valid sleep tracker to support data obtained from a sleep diary. Additionally, Tse and Hall (2008) successfully used a qualitative design as part of a larger mixed methods study to describe clients' perceptions of sleep interventions using semi-structured interviews. Researchers found that they were able to achieve a greater understanding of the clients' concerns and perceptions by completing a follow-up qualitative phase in the study (Tse \& Hall, 2008), thus reinforcing the significance of mixed methods research designs, client interviews, and the ability to capture the subjective experiences of participants. 
Ouellet and Morin (2007) were able to examine the efficacy of sleep interventions for individuals after TBI by subjectively measuring sleep and fatigue levels using semi-structured interviews. This study expands upon an earlier study conducted by the same researchers and also uses a single-case experimental design with multiple participants (Ouellet \& Morin, 2007). Results indicated positive treatment efficacy using a SSRD for multiple individuals with TBI (Ouellet \& Morin, 2007). However, the researchers failed to capture the full experience for individuals while participating in the sleep intervention study and did not include objective measures of sleep in the study, but researchers did recommend the use of both subjective and objective measures of sleep quality for future studies (Ouellet \& Morin, 2007). Even though this study could benefit from some modifications to improve the overall strength of the study's design and methodology, it yields very promising data for the effectiveness of multicomponent sleep interventions for individuals after TBI using a SSRD. These interventions could easily be implemented by an occupational therapist, and the suggested modifications would support a much stronger study.

\section{Summary of Existing Studies}

Overall, the majority of the existing studies that were discussed are quantitative studies that focus mainly on the subjective measurement of study outcomes, with the exception of the mixed methods studies and one qualitative study. Reliable objective measures are missing from many of these studies, as is the client experience that would be achieved through qualitative measures. Additionally, even though many of the studies were RCT studies that increased the generalizability of findings, only the studies using a SSRD were able to measure the effects of various interventions at the individual level. Therefore, the proposed study seeks to replicate aspects of those studies which exemplified a successful SSRD and/or mixed methods research 
methods while incorporating both subjective and objective measures to generate a comprehensive understanding of the occupational implications of sleep disruptions and the effectiveness of occupational therapy-directed sleep interventions.

\section{Summary of Literature Review}

Chapter two provided a summary of study constructs, a review of existing literature and measurement tools, and guiding theoretical frameworks to be utilized in a sleep intervention study guided by occupational therapy principles and practices. In summary, assisting clients with the development of healthy sleep habits is still an emerging area of practice and research in occupational therapy (Watson, Garden, Swedlove, \& Brown, 2014). However, occupational therapists are well-positioned to address sleep deficits for clients in many settings. The embedded mixed methods study, which was designed and implemented to understand the occupational implications of disrupted sleep and the effectiveness of various sleep interventions in a multicomponent sleep program for individuals after BI, is discussed in the next chapter. Chapter three will discuss the research methods of the current study, including the study design and population, measurement instruments, procedures and data analysis, and limitations of the study. 


\section{Chapter Three: Methods}

\section{Study Design and Rationale}

The embedded mixed methods design study evaluated the effectiveness of a multicomponent sleep program for individuals who were participating in a day treatment community re-entry program for adults after BI. Additionally, the study identified the occupational implications of disrupted sleep for this population. Several previous studies examined either quantitative or qualitative aspects of the relationship between sleep and functional participation. For example, Spira et al. (2012) studied the relationship between poor sleep quality and instrumental activities of daily living (IADL) for older women using wrist actigraphy and an IADL assessment to objectively measure performance, and O'Donoghue and McKay (2012) used semi-structured interviews to understand the influence of sleep disruptions on daily life for individuals with sleep apnea. However, no identified studies had examined both qualitative and quantitative aspects of sleep participation in the same study, which left many unanswered questions.

The research questions from the dissertation study could not be answered by either quantitative or qualitative methods alone, and a single data set was not sufficient to fully understand sleep disruptions within this population. Therefore, a mixed methods design was used to better understand both the effectiveness of various sleep interventions and the occupational implications of disrupted sleep for this population. An appropriate mixed methods design for this study was the embedded design.

With an embedded mixed methods design, data can be collected concurrently or sequentially in each strand of the study, with the secondary strand providing support for the primary strand (Creswell \& Plano Clark, 2011). In the dissertation study, qualitative and 
quantitative data were collected sequentially, with the primary focus on the quantitative strand and a secondary focus on the qualitative strand. In the first phase of the study, qualitative data were collected through semi-structured interviews with participants in order to support and enhance the outcomes in the quantitative phase by giving a more in-depth understanding (Creswell \& Plano Clark, 2011) of how disrupted sleep influenced participants' daily lives. Additionally, collecting rich qualitative data in the initial strand allowed the principal investigator to refine the recruitment procedures (Creswell \& Plano Clark, 2011) for the quantitative strand and rule out issues related to compliance, ensuring that participants were appropriate and motivated to participate in the sleep program. Collecting qualitative data in the initial phase of the study also supported the need for intervention with this population (Creswell \& Plano Clark, 2011). In a second phase, quantitative data were collected as the primary focus of the larger study in order to examine the effectiveness of a multicomponent sleep program. This was accomplished by measuring sleep participation and assessing functional outcomes related to sleep disturbance. Figure 5 provides a visual representation of the embedded mixed methods design utilized in the study.

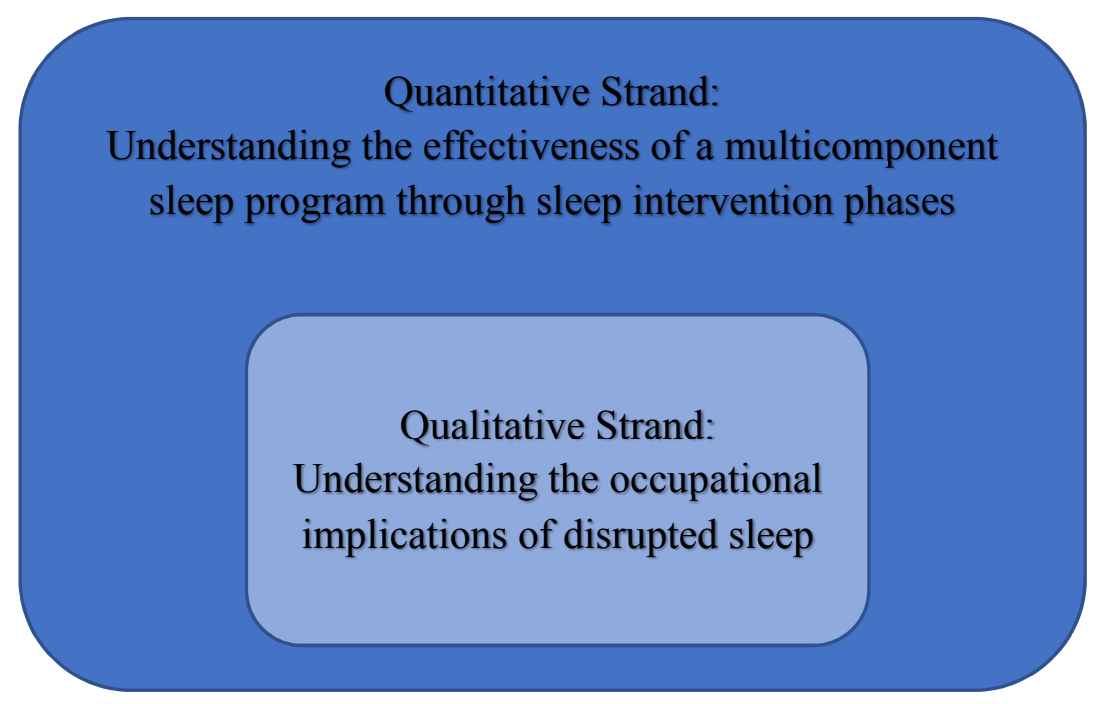

Figure 5. Graphic depiction of the embedded mixed methods design. 
Adding supplemental qualitative data to support the quantitative strand enhanced the larger study design and created a more in-depth understanding of the occupational implications of disrupted sleep and appropriate sleep interventions for this population.

\section{Study Population}

The specific population for the dissertation study included adults who were participating in an outpatient day treatment community re-entry program for individuals after BI. This population was selected due to the documented disturbances in sleep after BI, which can significantly affect functional performance in everyday activities (Bogdanov et al., 2017; Duclos et al., 2015; Mathias \& Alvaro, 2012; Ouellet \& Morin, 2004), including sleep. Evidence also suggests that individuals after BI have improved neurobehavioral and functional outcomes after participating in day treatment programs (Micklewright, Yutsis, Smigielski, Brown, \& Bergquist, 2011). Additionally, evaluating sleep in a client's home environment offered the benefit of addressing sleep concerns in a naturalistic setting that could be applied to everyday life. Liu et al. (2019) state that evaluating sleep in the home setting is just as important as the laboratory setting, and Smallfield and Lucas Molitor (2018) acknowledge that the role of occupational therapy in community-based settings is expected to increase, thus supporting the selection of an outpatient community-based program for the study setting in the dissertation study.

\section{Study Setting}

The specific program utilized for the dissertation study was the XXX Community ReEntry Program, an outpatient day treatment community re-entry program for individuals after BI related to illness, stroke, or accident. The program offers individual and group-based treatments to support program members in resuming functional roles within society $(\mathrm{XXX}, 2019)$ and operates Monday through Friday. Participants are typically enrolled in the program for an 
average of 7 weeks, and there are approximately 10 to 13 participants in the program at a given time (J. Ackland, personal communication, February 5, 2019). Many aspects of daily living are addressed by the program and are covered by the services provided in occupational therapy, physical therapy, speech therapy, psychology, neuropsychology, social work and case management, physician and nursing services, and nutrition services (XXX, 2019). Occupational therapy focuses primarily on IADLs, ADLs as needed, neuro re-education and neuro training, upper extremity strengthening and range of motion to improve functional participation, vision training, home visits, leisure pursuits, driver training, and community outings. There were three occupational therapists regularly working in the community re-entry program at the time of the study implementation (J. Ackland, personal communication, February 5, 2019). The principal investigator for the dissertation study was an occupational therapist who worked for the XXX organization but did not provide occupational therapy services in this program and only interacted with participants in relationship to the dissertation study. Every member in the community re-entry program received each of the previously mentioned services in the program, which also included participants in the dissertation study.

\section{Sampling}

The participants in the dissertation study included adult males and females who were participating in the community re-entry day rehabilitation treatment center at XXX Hospital. The demographics for participants in this study coincided with the admission criteria for the facility, which included individuals after BI who needed assistance with regaining functional roles within society $(\mathrm{XXX}, 2019)$ and who had previously participated in initial inpatient rehabilitation in the acute phase after BI. This population was selected due to the documented disturbances in sleep after BI, which can significantly affect functional performance in everyday 
activities (Duclos et al., 2015). For this population, purposeful sampling was used to ensure that the aim of the study was adequately addressed (O’Donoghue \& McKay, 2012). A convenience sample was also used based on the individuals who were participating in the program at the time, who had been in the program for less than 2 weeks to allow for maximum time to participate in the study, and who were willing to participate in the study. A convenience sample also maximized the number of participants who were included in the study. Participants who met the inclusion criteria for the study were recruited to participate in qualitative interviews. Interviews proceeded with clients of the XXX Community Re-entry Program until saturation was reached. These participants were also selected to participate in the quantitative strand of the study, with an expected maximum of six participants in the quantitative strand. There were three separate intervention phases of the study, which occurred in variable order for participants. Utilizing six participants would have allowed for two participants in each possible variation of the study interventions. To participate in either strand of the study, participants had to meet certain criteria.

\section{Inclusion and Exclusion Criteria}

Potential participants in the study had to be willing and able to participate in all phases of the study, including pre-screening assessments, sleep interventions, post-test assessments, qualitative data collection, and a brief one-month post-study follow-up via telephone call. Additional inclusion criteria for the study included being able to read, write, understand, and speak in English. Participants had to have a designated caregiver or a family member designated to provide continued support at home during participation in the program and in the dissertation study. Participants had to have no more than mild or mild-moderate cognitive impairment as determined by the pre-screening assessment conducted by the program's neuropsychology team, 
had to have at least "sub-threshold insomnia," or clinically significant insomnia symptoms, as determined by the sleep quality screening tool, and had to be willing and able to wear a sleep tracking device on the wrist during nighttime sleep. Individuals were excluded from the study if they had chronic pain issues with narcotic use for pain management, had severe spasticity that affected functional performance, and had a household member who regularly affected sleep at baseline (e.g. an infant with unstable sleep patterns). Factors related to pain, spasticity, and household members affecting sleep were added into the exclusion criteria because these factors could significantly affect a person's ability to achieve a good night of sleep, despite sleep interventions. Sleep studies conducted by Ouellet and Morin (2007) and Gutman et al. (2017) considered similar factors for inclusion and exclusion criteria. Several instruments were used in the study to evaluate cognition, sleep participation, and factors associated with sleep performance.

\section{Measurement Instruments}

When a client acknowledges having sleep problems during an occupational therapy assessment or treatment session, it is important to identify factors related to the client's sleep habits and sleep participation. Occupational therapists should do a full assessment to gather details about the client's sleep patterns, his or her behaviors related to sleep, and the sleep environment (Green \& Hicks, 2015). There are a variety of sleep assessments that can be utilized by occupational therapists and other health care professionals to further evaluate sleep and to measure outcomes related to sleep participation (Green \& Hicks, 2015). The Insomnia Severity Index, the Sleep Hygiene Index, the Functional Outcomes of Sleep Questionnaire, the Epworth Sleepiness Scare, and the Consensus Sleep Diary were used to evaluate sleep in the dissertation study and are discussed in greater detail in following sections. Prior to initiating a 
study, pre-screening assessments should be conducted to gather baseline information about potential participants in a study. The pre-screening assessments for the dissertation study assessed baseline sleep and cognitive function.

\section{Pre-Screening Data Collection Instruments}

A battery of pre-screening assessments was implemented to ensure that individuals had the cognitive capacity to actively participate in the study and to provide baseline information on participants' sleep performance. Pre-screening assessments included the Trail Making Test, Part $\mathrm{B}$, and the Insomnia Severity Index.

The Trail Making Test, Part B. The Trail Making Test was developed in 1944 as part of the Army Individual Test Battery. It is commonly used in practice and research because it is sensitive to determining cognitive impairment (Kortte, Horner, \& Windham, 2002). The test has two parts. Part A requires participants to draw lines connecting 25 numbers in consecutive order, and Part B requires drawing lines that alternate between numbers and letters in consecutive order. Each task is completed as quickly as possible, and scores are based on the time taken to complete the test (Kortte et al., 2002). Part B is more sensitive to deficits in flexibility and executive functioning (Bastug et al., 2013; Kortte et al., 2002). The written format requires intact visual and motor skills, but there is an oral version that was developed in 1994 (Bastug et al., 2013). Bastug et al. (2013) found the oral version of the Trail Making Test, especially Part B, to be a valid tool for measuring different levels of cognitive impairment. Trail Making Test, Part B has been validated by over 40 normative studies, according to Strauss, Sherman, and Spreen (2006). Wagner, Helmreich, Dahmen, Lieb, \& Tadić (2011) found the Trail Making Test, Part B to be suitable for testing executive functioning, with retest reliability scores between 0.86 and 0.94 . Trail Making Test, Part B was selected for the dissertation study 
because it was a cognitive screening tool that was utilized at the site for the dissertation study, where cognitive functioning was assessed by neuropsychology as part of the admission screening process (see Appendix A for the Trail Making Test, Part B score sheet). After assessing baseline cognition, baseline sleep performance was assessed using the Insomnia Severity Index.

Insomnia Severity Index. The Insomnia Severity Index is an insomnia screening tool that is designed to assess emotional distress and functional impairments associated with decreased quality of sleep (Morin, 1993). The assessment measures difficulties falling asleep and maintaining sleep, waking early, sleep satisfaction, perceptions of the impairment, and how disruptions in sleep affect daily functioning (Mairs \& Mullan, 2015). The Insomnia Severity Index also identifies sub-threshold insomnia, which indicates lower grade sleeping problems (Morin, 1993). Total scores on the Insomnia Severity Index range from zero to 28, with a score of zero to seven indicating "no clinically significant insomnia," eight to 14 indicating "subthreshold insomnia," 15 to 21 indicating "moderate insomnia" and 22 to 28 indicating "severe clinical insomnia" (Morin, 1993). Participants in the dissertation study had to score greater than seven on the Insomnia Severity Index, indicating clinically significant sleep problems. The Insomnia Severity Index has demonstrated good concurrent and predictive validity and internal consistency, with Cronbach's alpha $=0.74$ to 0.78 (Mairs \& Mullan, 2015). Moscou-Jackson, Allen, Smith, and Haywood (2016) also acknowledge that the Insomnia Severity Index demonstrates good internal consistency among items in each factor-scale with Cronbach's alpha $=0.85$ to 0.87 . The Insomnia Severity Index (Smith \& Wegener, 2003) was selected for the dissertation study because it is a brief screening tool that offers data regarding the clinical significance of sleep problems (see Appendix B for the Insomnia Severity Index score sheet). Information from this assessment tool was used to determine eligibility to participate in the 
dissertation study and to gain baseline data regarding participants' sleep quality and sleep patterns prior to initiating the study. Additionally, the Insomnia Severity Index was used to calculate sleep quality after participating in the sleep interventions and again at a one-month follow-up to determine the continued effectiveness of the sleep interventions in improving sleep quality after one month. Other sleep assessments were used to evaluate sleep outcomes.

\section{Outcome Measures Data Collection Instruments}

During the quantitative phase of the study, subjective sleep data were collected using the Insomnia Severity Index, the Sleep Hygiene Index, the Functional Outcomes of Sleep Questionnaire, the Epworth Sleepiness Scale, and the Consensus Sleep Diary, and objective sleep data were collected using the Fitbit Inspire HR. Qualitative data were collected using semi-structured interviews.

The Sleep Hygiene Index. The Sleep Hygiene Index is a self-administered index of 13 items intended to assess the presence of behaviors or environmental factors that interfere with good sleep hygiene. Participants indicate how frequently they engage in each behavior, indicating responses of always, frequently, sometimes, rarely, or never. Items are summed to give a global score of sleep hygiene, and higher scores indicate more maladaptive sleep hygiene habits (Mastin, Bryson, \& Corwyn, 2006). Items on the Sleep Hygiene Index were derived from the diagnostic criteria for inadequate sleep hygiene in the American Sleep Disorders Association's International Classification of Sleep Disorders from 1990 (Mastin et al., 2006). Reliability of the Sleep Hygiene Index yields a Cronbach's alpha score of 0.66 and good testretest reliability, $r(139)=0.71, p<.01$ (Mastin et al., 2006). The Sleep Hygiene Index is positively correlated, $p<.01$, with the diagnostic criteria for inadequate sleep hygiene, with Pearson $r$ values ranging from 0.371 to 0.458 (Mastin et al., 2006). It was selected for the 
dissertation study as a quick assessment of baseline sleep hygiene habits prior to administration of sleep interventions (see Appendix C for the Sleep Hygiene Index score sheet). The Functional Outcomes of Sleep Questionnaire was used to assess how functional activities were affected by a participant's sleep habits.

The Functional Outcomes of Sleep Questionnaire. The Functional Outcomes of Sleep Questionnaire-30 is a self-administered assessment that takes approximately 15 minutes to complete. It is designed to assess the effects of disorders of excessive sleepiness (DOES) on activities related to normal daily functioning and quality of life (Weaver et al., 1997).

Participants are instructed to rate items on a four-point rating scale, with responses ranging from no difficulty, a little difficulty, moderate difficulty, and extreme difficulty, with lower scores indicating great dysfunction. The original version contained 74 items, which were used for the basis of initial psychoanalysis (Weaver et al., 1997). Many items were dropped for various reasons, making a more concise assessment of 30 items. Construct validity is demonstrated with subscale-to-global Functional Outcomes of Sleep Questionnaire score correlations ranging from $r=0.78-0.86$ and a Cronbach's alpha score of 0.95 for the 30 -item scale (Weaver et al., 1997). Test-retest reliability ranged from $r=0.81$ to $r=0.90$ for individual subscales, and internal consistency was good with a reliability coefficient of 0.70 (Weaver et al., 1997). This assessment was selected for the dissertation study in order to understand the occupational implications of disruptions in sleep for individual participants and to track changes in functional performance between sleep intervention phases (see Appendix D for the Functional Outcomes of Sleep Questionnaire score sheet). The Epworth Sleepiness Scale was used to assess daytime sleepiness during the study. 
The Epworth Sleepiness Scale. The Epworth Sleepiness Scale is commonly used to assess daytime sleepiness and to measure sleep propensity (Johns, 1991). It includes a list of eight situations where a person might fall asleep, and the person rates his or her chance of dozing on a zero to three-point scale. Scoring is based on the participant's usual way of life, and participants are asked to estimate how each situation might affect them even if they have not been in that situation recently (Johns, 1991). The numbers selected on each situation are added together to give a total score between zero and 24 , with higher scores indicating increased daytime sleepiness (Johns, 1991). Criterion validity was demonstrated using a one-way ANOVA that yielded significant differences in Epworth Sleepiness Scale scores between multiple diagnostic groups, $F=50.00 ; d f=6,173 ; p<.0001$, and concurrent validity was demonstrated when there was a significant correlation between Epworth Sleepiness Scale scores of overnight polysomnography for sleep latency at night, $r=-0.379, n=138, p<.001$ (Johns, 1991). The Epworth Sleepiness Scale was selected for the dissertation study to evaluate daytime sleepiness and to assess participants' safety on a weekly basis while participating in the intervention phases (see Appendix E for the Epworth Sleepiness Scale score sheet). The Consensus Sleep Diary was used to measure various factors associated with each night of sleep for participants.

The Consensus Sleep Diary. The sleep diary is considered the gold standard in subjective sleep measures, but there is a lack of standardization of sleep diaries and minimal psychometric testing (Carney et al., 2012). The Consensus Sleep Diary was developed based on the input of a large number of sleep experts and with qualitative patient input, partly to address the lack of standardization of sleep diaries. A workgroup developed the Consensus Sleep Diary primarily for insomnia research but also to be utilized in the clinic and in research for all sleep disorders (Carney et al., 2012). The core Consensus Sleep Diary is completed in the morning, 
consists of nine items, and fits on the front of one sheet of paper, and the expanded Consensus Sleep Diary is completed in the morning and before bed, includes 15 items, and fits on the front and back of one sheet of paper. Both versions allow for 7 days of tracking. Both versions yield information about relevant sleep metrics, including sleep onset latency, wakefulness after initial sleep onset, total sleep time, total time spent in bed, sleep efficiency, and sleep quality (Carney et al., 2012). There has been no formal reliability or validity testing of the Consensus Sleep Diary, which is common for sleep diaries (Carney et al., 2012). The core version of the Consensus Sleep Diary was selected for the dissertation study because it is thorough, yet brief. It is also sensitive to change on a daily basis and gives daily data regarding sleep performance, which was important to assess during this study over the course of various sleep interventions and from dayto-day. Data collected from the Consensus Sleep Diary was used to calculate sleep onset latency, total sleep time, sleep efficiency, and the number of awakenings for the dissertation study (see Appendix F for the Consensus Sleep Diary worksheet). While sleep diaries have occasionally been noted to overestimate the severity of sleep disturbances, they are useful tools to complement commercial sleep trackers in a multi-method approach to evaluating sleep (Smith \& Wegener, 2003).

Fitbit Inspire HR. The Fitbit Inspire HR, which is similar to the recently retired Fitbit Alta HR, is a wristband that measures activity and sleep by tracking the intensity and frequency of movements. Like the Fitbit Alta HR, the Fitbit Inspire HR continuously monitors a person's heart rate variability and heart rate, and sleep cycles are estimated using the heart rate variability and movement data after a night of sleep (Kosecki, 2017). Fitbit tracking devices can measure total sleep time, sleep latency, sleep efficiency, and the number of awakenings (Kang et al., 2017). Data from a Fitbit are uploaded to an online account, where sleep metrics are calculated 
(Kang et al., 2017). Because the Fitbit Inspire HR was a newly released device in 2019, there was no identified evidence documenting the effectiveness of estimating sleep using this device. However, the Fitbit Alta HR is a widely used commercial sleep tracker and can be used interchangeably with a sleep diary for long-term monitoring to obtain data regarding total sleep time and sleep onset latency, according to Liu et al. (2019). Cook et al. (2018) also acknowledge that the Fitbit Alta HR has the potential to estimate sleep when compared to standard actigraphy in non-sleep laboratory settings. Additionally, other Fitbit devices, such as the Fitbit Flex, have estimated sleep well enough to show changes between variable conditions (Morhardt et al., 2017) and can still be useful as commercial devices to track sleep patterns, especially when complemented with the use of a sleep diary (Kang et al., 2017).

Kang et al. (2017) also discussed how the Fitbit Flex demonstrated high inter-device reliability for total sleep time with an intraclass correlation coefficient value of 0.974 for the Fitbit Flex and polysomnography (Kang et al., 2017). Even though several studies document the utility of the Fitbit Flex in regards to sleep tracking (Cook, Prairie, \& Plante, 2017; Kang et al., 2017; Morhardt et al., 2017), the Fitbit Inspire HR was selected for the dissertation study due to its ability to track heart rate and heart rate variability. Because the Fitbit Inspire HR tracks heart rate variability, it is more reliable in detecting restlessness and short periods of wakefulness, like the Fitbit Alta HR (Kosecki, 2017). Pertinent data collected from the Fitbit Inspire HR included sleep onset latency, total sleep time, sleep efficiency, and the number of awakenings. Total sleep time was calculated directly from the Fitbit data. Sleep onset latency was calculated using the time at which the participant attempted to fall asleep from the Consensus Sleep Diary and the time that the participant fell sleep from Fitbit Inspire HR data, sleep efficiency was calculated from the total sleep time from the Fitbit Inspire HR data and total time in bed taken from the 
Consensus Sleep Diary data, and the number of awakenings was collected directly from Fitbit data (see appendix $G$ for the Sample Fitbit Output Data sheet). In addition to quantitative sleep measures, qualitative measures were used as a supplement to further strengthen study findings in regards to the occupational implications of disrupted sleep.

Semi-structured interviews. The qualitative phase of the dissertation study included semi-structured interviews with participants to determine the occupational implications of disruptions in sleep and to gain additional information about the factors influencing participants' sleep. The use of semi-structured interviews ensured the collection of rich data that was unique to participants in the study (O’Donoghue \& McKay, 2012) and supported the need and recruitment of participants in the quantitative strand. For example, O’Donoghue and McKay (2012) used semi-structured interviews to explore the experiences of people living with obstructive sleep apnea. This type of data collection allowed the researchers to obtain meaningful data that could not be captured by quantitative sleep measures alone, thus highlighting a benefit of using mixed methods research designs. Questions on the interview guide were used to invoke responses and guided discussions in attempt to understand the personal experiences of how disruptions in participants' sleep influenced their daily occupational engagement (see Appendix $\mathrm{H}$ for the Interview Schedule). The procedures section includes information on how data were collected and specific details regarding the design of both the quantitative and qualitative strands of the study.

\section{Procedures}

Participants in the study were already members of the day treatment community re-entry program, where each member of the program was given the TBT, Part B as a part of their initial neuropsychology testing upon entry into the program. During the study period, each member of 
the program was also be given the Insomnia Severity Index after admission into the community re-entry program. Additionally, staff members in the community re-entry program received education on the dissertation study.

\section{Preparatory Procedures}

Prior to implementation of the study, the principal investigator obtained approval from the Internal Review Board from the facility and from her educational institution. After approval was obtained from both sources, staff at the community re-entry site received brief education and training from the principal investigator to educate them on the purpose of the study and how they could facilitate the study process. Staff members were also educated to refer to the principal investigator if questions arose from staff members, caregivers, or participants in the program. The lead occupational therapist in the program informed the principal investigator of new admissions to the program. The principal investigator also had access to review initial occupational therapy and neuropsychology evaluation notes to determine if new program members met the inclusion criteria for the study.

Program members who met the inclusion criteria for the dissertation study met with the principal investigator to review the goals and general guidelines for participation in the study. Individuals who agreed to participate in the study then reviewed and signed a consent form and a Health Insurance Portability and Accountability Act form in order to participate in the study (see Appendix I for the Participant Consent Form and Appendix $\mathbf{J}$ for the Health Insurance Portability and Accountability Act Form). Demographic information was then collected from participants regarding the participant's age, gender, factors affecting sleep performance, and pre-existing sleep habits and patterns (see Appendix K for the Demographic Data Collection Form). Caregivers, or designated family members, were also contacted before the scheduled meeting 
with potential participants to receive brief education regarding the proposed study. Caregivers needed to provide daily reminders to participants and signed a form agreeing to provide these reminders and to receive daily reminders from the principal investigator in the form of text message and/or e-mail (see Appendix L for the Caregiver Agreement Form). Because designated family members and caregivers were already significantly involved in reviewing and assisting with adherence to daily activities and exercises and were expected to regularly review community re-entry program members' memory and activity notebooks, asking them to provide these reminders required minimal effort in addition to the current expectations of the community re-entry program. Once a community re-entry program member agreed to participate in the study and appropriate forms were signed, participants were educated on the importance of sleep to gain buy-in from participants. Participants were issued a one-page handout reviewing this information for future reference (see Appendix M for the Sleep Is Important handout). Steps were then taken to initiate the qualitative strand of the study.

\section{Qualitative Measures}

The first phase of the study was the qualitative strand. The principal investigator collected meaningful data from participants using semi-structured interviews.

Study design. Semi-structured interviews were completed with participants during individual sessions. Participants had face-to-face meetings with the principal investigator on a one-on-one basis to participate in guided conversations with open-ended questions. The interview included questions that addressed sleep-related factors and the occupational implications of disrupted sleep in order to understand participants' experiences and the meaning of disrupted sleep for each participant in the study. 
Data collection. During the interview process, data were collected from the responses to interview questions. Participants were also asked additional probing questions in order to clarify and/or elaborate on information presented and to gather more meaningful and rich data from each participant. To preserve confidentiality and anonymity of participants, pseudonyms were assigned to participants and used throughout the interview process and in publication of study results. Data from interviews was audio recorded and transcribed verbatim into a narrative format within 24 hours of completion of the interviews, and recordings were imported into the NVivo qualitative data analysis system (QSR International, 2018). Some initial data were also collected prior to initiating the study.

To increase the validity of the interview guide prior to use in the current study, the principal investigator practiced administering questions to a small number of individuals with designated sleep deficits. This process allowed the researcher to assess responses to questions, to potentially re-word or revise questions on the interview guide or during the interview process, and to get feedback from others (Chenail, 2009). Prior to initiation of the study, the principal investigator also wrote about her own thoughts regarding sleep and how disruptions in sleep might influence daily life activities in order to consciously acknowledge any perceptions regarding the phenomenon being studied. This process is recommended by Moustakas (1994) when utilizing phenomenological research approaches. Information was also collected from the principal investigator through the use of reflexive journaling, which allowed the researcher to record thoughts and ideas before and after the completion of the interviews (Chenail, 2009). In addition to self-reflections regarding the interview process, site observations and personal impressions were also recorded, which is also recommended by Moustakas in the first step of data analysis. 
Data analysis. As previously discussed, the first step in data analysis involves the identification of the researcher's perspective, which was completed through reflexive journaling (Moustakas, 1994). The next step in data analysis involves reducing the narratives of participants (Moustakas, 1994). Transcribed narratives were read repeatedly by the principal investigator in order to understand the meaning of the statements in the participants' words and within context. Significant statements were highlighted on the NVivo qualitative software system (QSR International, 2018). These significant statements were identified during repetitive reading based on their relationship to occupational engagement or a lack of occupational engagement. Examples included statements related to a desire to participate in occupations or barriers to participating in occupations. Significant statements were read multiple times and eventually clustered based on thematic labels (Moustakas, 1994), which were tracked in the qualitative software system.

After analyzing and interpreting the meaning of the phenomenon in the original texts, final core themes were identified to represent the participants' experiences (Moustakas, 1994). These core themes were referred back to the original texts for validation (Moustakas, 1994). Additionally, individual textural descriptions of each participant's experience were formulated and taken verbatim from participant's transcripts after review of significant statements and themes (Moustakas, 1994). Thematic labels were also given to the identified core themes, and a description of the meaning and the essence of the experience related to occupations and disrupted sleep after BI (Moustakas, 1994). All data were tracked and coded in the qualitative software system. Several techniques were employed to ensure the trustworthiness of the data collected. An outside reader on the principal investigator's dissertation committee read and reviewed the data to ensure the accuracy of the descriptions and the identified themes and labels 
(Moustakas, 1994). The process of triangulation was also be used to confirm the data received during qualitative interviews by comparing it to data collected from the assessments completed in the quantitative phase (Portney \& Watkins, 2015). An audit trail, which is part of the reflexive journaling previously discussed, was also created to clearly describe and document the thought process used by the principal investigator while interpreting qualitative data (Portney \& Watkins, 2015). Member checks were performed with each participant to validate participants' interpretations of the data and to ensure accuracy (Portney \& Watkins, 2015). After completion of the qualitative strand, participants were recruited to participate in the quantitative strand of the study.

\section{Quantitative Measures}

The second phase of the study, and the primary focus of the larger study, was the quantitative strand. The principal investigator collected baseline data and both objective and subjective data that tracked the effectiveness of various sleep interventions throughout the intervention phases of the study using a SSRD.

Study design. A single-subject research design (SSRD) was selected for the quantitative strand of the study, in part, due to the small sample size for the study. This type of study is also very useful for evaluating the efficacy of an intervention within a group of individuals under controlled conditions, and information obtained from SSRD studies can be applied to clinical practice (Burgard, Foster, Kraus, \& Radel, 2011). A multiple baseline design across subjects was used, with the same sleep interventions applied to multiple participants throughout the intervention phases (Ottenbacher, 1986). Baseline objective sleep data were collected for each participant in phase A of the study using the Fitbit Inspire HR and the Consensus Sleep Diary until baseline data stability was achieved (Ottenbacher, 1986). Baseline stability was calculated 
using sleep efficiency data from the Consensus Sleep Diary, and stability was achieved once $80 \%$ of the data in the baseline phase fell within $15 \%$ of mean of all data points for the baseline phase (Lobo, Moeyaert, Baraldi Cunha, \& Babik, 2017). Participants who did not reach baseline stability within two weeks of admission to the community re-entry program could have been excluded from the study due to the limited amount of time that participants were in the program. After participants achieved baseline stability, sleep interventions were initiated.

There were three sleep intervention phases, with interventions labeled as intervention B, intervention C, or intervention D. The interventions occurred in variable order for each participant (see Figure 5 for a graphic depiction of the possible variations of order for intervention phases with at least three participants). Changing the order in which participants received the interventions, referred to as counter-balancing of interventions, helped to reduce the risk of order effect, which happens when behavior changes could occur when prior treatments interact with current treatments (Ottenbacher, 1986). If there were more than three participants in the study, additional participants would have been randomly assigned to a sequence of interventions based on the patterns of interventions depicted in Figure 6.

\begin{tabular}{lllll}
\hline & $\begin{array}{l}\text { Baseline } \\
\text { Phase A }\end{array}$ & $\begin{array}{l}\text { Intervention } \\
\text { Phase 1 }\end{array}$ & $\begin{array}{l}\text { Intervention } \\
\text { Phase 2 }\end{array}$ & $\begin{array}{l}\text { Intervention } \\
\text { Phase 3 }\end{array}$ \\
\hline Participant 1 & $\begin{array}{l}\text { No } \\
\text { Intervention }\end{array}$ & Intervention B & Intervention B + C & Intervention B + C + D \\
\hline Participant 2 & $\begin{array}{l}\text { No } \\
\text { Intervention }\end{array}$ & Intervention C & Intervention C + D & Intervention C + D + B \\
\hline Participant 3 & $\begin{array}{l}\text { No } \\
\text { Intervention }\end{array}$ & Intervention D & Intervention D + B & Intervention D + B + C \\
& & & \\
\hline
\end{tabular}

Figure 6. Sample order of interventions for three participants.

Each of the three intervention phases occurred for 1 week at a time and built upon the previous weeks' interventions. Daily data came from the sleep diary and the Fitbit Inspire HR tracker and were collected by the principal investigator on a bi-weekly basis. 
Data collection. To evaluate the utility of the Fitbit Inspire HR, a pilot study involving two clinicians was completed to compare daily data from the Fitbit device to the Consensus Sleep Diary for one week. These data supported the use of these outcome measures in the dissertation study. To initiate data collection in the dissertation study, baseline data were collected during the initial meeting with each participant after the initial education session and signing of consent forms. Participants completed the Sleep Hygiene Index, the Functional Outcomes of Sleep Questionnaire, and the Epworth Sleepiness Scale during this first session. Participants were also issued the Fitbit Inspire HR and sleep diary forms to begin baseline sleep data collection that night. Participants were educated on how to properly use and care for the Fitbit device prior to use (see Appendix N for Fitbit Inspire HR Instruction Guide) and were instructed to bring the Fitbit with them daily to the community re-entry program while participating in the study period. Data were continuously collected daily during the quantitative phase of the study using the Fitbit Inspire HR and the Consensus Sleep Diary. During the sleep intervention phases, participants also reviewed the Daily Intervention Checklist every evening at bedtime as a reminder and completed the form in the morning with the Consensus Sleep Diary as verification that the prescribed interventions were completed (see Appendix O for Sample Daily Intervention Checklists). The checklist form was updated to reflect the current intervention phase for each participant, with an updated form issued to each participant weekly.

After completion of each of the intervention phases, the principal investigator met with participants to re-administer the Functional Outcomes of Sleep Questionnaire and the Epworth Sleepiness Scale to evaluate sleepiness and to determine how potential changes in sleep participation affected daily life activities after an intervention phase. The Insomnia Severity Index was re-administered after all intervention phases were completed and at a 1-month follow- 
up in order to evaluate levels of insomnia and sleep quality after participating in the interventions. The principal investigator regularly met with participants on a bi-weekly basis to collect data throughout the study, to check in with participants, and to educate and instruct participants on the next intervention phase (see Appendix P for Intervention Phases Educational Handouts). The principal investigator was available daily via telephone or through e-mail contact for any potential questions or concerns from the participants, participants' caregivers, and the community re-entry staff.

Staff working in the program, particularly occupational therapists, were educated to provide daily reminders, Monday through Friday, to ensure that participants were tracking data and participating in the assigned sleep interventions during the intervention phases. Participants kept all handouts, daily checklists, and sleep diary forms in the memory and activity notebook, which was issued to each member in the community re-entry program to organize daily activities and to provide pertinent resources for members and caregivers to utilize throughout participation in the community re-entry program. Occupational therapists working in the community re-entry program were educated to review the participants' notebooks daily. Monday through Friday, to verify that appropriate forms were completed. Caregivers, or designated family members, provided daily reminders to ensure that appropriate forms were completed on weekends and other days that participants were not in the program. Caregivers also provided daily reminders during the intervention phases of the study.

Interventions. There were three intervention phases in the dissertation study, which were completed by each participant in variable order. Intervention B was the stimulus control intervention. During the intervention B phase, participants were instructed to adhere to the following stimulus control instructions from Bootzin and Perlis (2011): 
1. Lie down and go to bed only when feeling sleepy.

2. Use the bed and bedroom for sleep and sexual activity only. Do not use your cell phone or watch television in bed.

3. If unable to fall asleep or awake in bed for more than 10 minutes, get up and go into another room to participate in calming activities. Go back to bed when feeling sleepy.

4. Set an alarm clock to get up at the same time each morning, regardless of bedtime.

5. Avoiding napping during the day.

During the intervention $\mathrm{C}$ phase, participants were instructed to adhere to the following behavioral and occupational sleep hygiene instructions from Posner and Gehrman (2011):

1. Exercise in the late afternoon or early evening (before dinner). As an alternative, take a warm bath or shower one to two hours before bedtime.

2. Eat a light snack, such as crackers, bread, or fruit, before bedtime. Avoid sugary snacks.

3. Avoid too many liquids before bedtime, drinking no more than 6-7 ounces in the last four hours before bedtime. Drink a small cup of chamomile tea before bedtime.

4. Avoid caffeinated products within 6 hours of bedtime.

5. Avoid alcohol and limit nicotine use, especially in the evening hours.

During the intervention D phase, participants were instructed to adhere to the following environmental sleep hygiene instructions from Posner and Gehrman (2011):

1. Use earplugs or some form of white noise (e.g., fan or humidifier, white noise apps) to mitigate noise.

2. Avoid co-sleeping with pets (if applicable).

3. Make sure that bedding and pillows are clean and comfortable. 
4. Keep the bedroom cool, between 65 and 68 degrees if possible or by using a fan.

5. Keep the bedroom dark by covering windows with a dark blanket or sheet, the use of blackout blinds or dark curtains, or by wearing an eye mask.

Earplugs, eye masks, and chamomile tea were issued to each participant, and assistance was provided to install white noise apps on a phone or tablet as applicable. The quantitative phase of the study was completed after all participants completed each of the three intervention phases. After data were collected from the quantitative phase, data analysis occurred.

Data analysis. The dissertation study was a SSRD, which used repeated measures to evaluate behavioral responses over a period of time (Ottenbacher, 1986). For this type of study, the "statistical rule of thumb" is the two standard deviation band method (Ottenbacher, 1986). This method involves an assessment of the variability within the baseline phase through the calculation of the mean and standard deviation of data points within various phases (Portney \& Watkins, 2015), and can be used with even a small number of baseline data points or when there is a high degree of variability in baseline data (Ottenbacher, 1986). These data are used to assess whether or not there is significant change from the baseline to the intervention phases (Portney \& Watkins, 2015). Statistical significance is indicated when two consecutive points fall outside of the two standard deviation band (Portney and Watkins, 2015 and Ottenbacher, 1986). Another means for statistical analysis with the SSRD is the C-statistic, which estimates trends in timeseries data (Ottenbacher, 1986). With the C-statistic, calculations start with baseline data. If there is no significant trend within baseline data, baseline and intervention data are combined and the $\mathrm{C}$-statistic is calculated to determine if there is a significant change in trend across phases (Ottenbacher, 1986). The C-statistic provides a numeric estimate of stability (Ottenbacher, 1986). While statistical analysis offers a more concrete way to assess variability, the focus in 
SSRD studies is more on clinical relevance than statistical significance (Burgard et al., 2011).

Therefore, visual analysis is the most frequently used means of analysis with a SSRD

(Ottenbacher, 1986).

With visual analysis of the data, researchers are able to determine the clinical significance of the study's outcomes by measuring the level, trend, variability, and slope of the data (Portney \& Watkins, 2015), often using line graphs. Level is described as the magnitude of performance (Portney \& Watkins, 2015). It is measured by comparing the value of the target behavior at the last data point of one phase with the value at the first data point of the next phase or by comparing the mean values of the target behavior within phases (Portney \& Watkins, 2015). Changes in level represent rising or falling in performance after introduction of the intervention (Ottenbacher, 1986). The trend of the data is the direction in which data points are progressing and can be described as accelerating or decelerating (Ottenbacher, 1986). The slope refers to the angle of the trend (Ottenbacher, 1986), which is also the rate of change in the target behavior (Portney \& Watkins, 2015). Variability refers to the amount of fluctuation of data points in a series (Ottenbacher, 1986). Using visual analysis does not always offer strong reliability, but methods are more reliable when trend is assessed by drawing a straight line to identify the rate of change (Portney \& Watkins, 2015). The most popular method of visual analysis involves the use of a celeration line (Portney \& Watkins, 2015), which is intended to demonstrate a trend line to the data points in a phase that indicates whether the data is accelerating, decelerating, or stationary (Ottenbacher, 1986). After collecting both quantitative and qualitative data, methods were employed to integrate the data. 


\section{Integration of Quantitative and Qualitative Data}

After evaluating the data from both qualitative and quantitative strands of the study, data were integrated using the inference process in order to gain a better understanding of the experience of sleep and to understand the occupational implications for disrupted sleep for this population. Sequential mixed data analysis was used for this study, considering that the qualitative and quantitative aspects of the study occurred in sequential order (Teddlie \& Tashakkori, 2009). Data collected in each phase were linked based on the participants' sleep participation from the quantitative strand and their explanation of sleep-related factors in the qualitative strand. Categories and themes were also identified during the quantitative phase and then confirmed or compared to the themes identified in the qualitative phase with the desire to create construct validation (Teddlie \& Tashakkori, 2009). Meta-inferences were drawn at the end of the study to determine if the qualitative data provided a better understanding of the research problem than just the quantitative data alone (Creswell \& Plano Clark, 2011). Mixed data were organized and displayed in a graphic image that linked quantitative results to qualitative themes in order to explain and understand the meaning of the study results and to have a better understanding of the functional implications of decreased sleep for the BI population.

\section{Anticipated Limitations}

Some limitations with the quantitative strand were the small sample size and the use of a SSRD limited generalizability of results across populations and settings. The timeframe of the study was also bound by the limited amount of time that participants are in the community reentry program, which limited the allowable time for participants to be in the dissertation study. The risk of co-interventions provided by other health care providers working in the community 
re-entry program was also a potential limitation of the study. Additionally, the sample for both strands was a convenience sample of individuals at a specific site and after BI, further limiting generalizability of results. For the quantitative strand, participants might have incorrectly filled out the sleep diary and might have potentially been untruthful in both strands of the study. The Fitbit Inspire HR was also a new device at the time of study implementation, with limited application in research and practice.

\section{Anticipated Ethical Issues}

Prior to initiating the dissertation study, the research proposal was sent to the Internal Review Boards for XXX and for Nova Southeastern University. The identity of participants was protected by the use of codes instead of participant's real names. Hard copies of data collected during the study were kept for 3 years after the completion of study and were stored in a locked filing cabinet. Electronic copies and audio files were kept on the principal investigator's computer, which was password-protected. Because the sleep interventions were noninvasive, there were no anticipated ethical concerns within the intervention phases of the study. Information received through a review of participants' medical records was protected by the Health Insurance Portability and Accountability Act.

\section{Summary of Methods}

The embedded mixed methods design study was designed to determine which sleep interventions were most beneficial for individuals after BI and to determine the occupational implications of sleep disturbance for this population. Integrating the data helped to better understand how disruptions in sleep affected the daily lives of individuals after BI, and offered a more holistic understanding of sleep disturbance that can easily be addressed by occupational therapy practitioners. 


\section{Chapter Four: Results}

The focus of this chapter is to present the results of the qualitative and quantitative strands as well as the integration of both strands of the mixed methods dissertation study. During the qualitative strand of the study, semi-structured interviews were completed with all participants in the initial phase of the study. Codes were identified and applied to significant statements from participants, and then core themes with applicable thematic labels that related to all participants were identified. Finally, textural and structural descriptions of the meaning and essence of the experience of disrupted sleep and the occupational implications of sleep disturbance after BI were presented. During the second and primary phase of the study, quantitative analysis was completed through extensive descriptive and visual analysis techniques of level, trend, variability, and slope using multiple sleep-related outcome measures. Additional sleep assessments measured the level of insomnia, sleepiness, and functional outcomes related to sleep disturbance at baseline and throughout the study. The quantitative study design was a SSRD that was utilized during a multicomponent sleep program with each of the participants to determine which factors affected sleep and the occupational implications of disrupted sleep after BI. The same participants were used for both qualitative and quantitative strands of the study.

\section{Participant Characteristics}

During the study period, 13 people were admitted into the community re-entry program and were screened for the dissertation study between August 2019 and March 2020. Of those screened, seven people who met the inclusion criteria for identified sleep deficits were presented with information regarding the research study by the principal investigator. Three of those individuals did not meet other study inclusion criteria. One did not have family support at home, and two did not meet the cognitive inclusion criteria based on results of the cognitive screening 
tool completed by neuropsychology. The remaining individual declined to participate in the study for personal reasons. Three individuals agreed to participate in the study, reviewed and completed all necessary consent and demographic forms, and completed initial assessments prior to participating in the semi-structured interviews.

Each of the three participants were male, and each had experienced a cerebrovascular accident (CVA) prior to being admitted into the community re-entry program and being enrolled into the study. They were all married, their wives were present during the initial meetings and interviews, and the wives each agreed to be the designated caregiver and a contact person throughout the study. The mean age for the participants was 51.3 years and the median age was 50 years. The mean time between experiencing the CVA and initiating participation in the study was 48.3 days. See Table 1 for additional participant characteristics.

During the research study, each of the study participants partook in a multicomponent sleep program during the quantitative phase of the study to explore the identified research questions. There were two quantitative research questions addressed in the dissertation study.

\section{Quantitative Research Questions}

1. Does participation in a multicomponent sleep program improve sleep participation for adults after BI who are participating in a day treatment community re-entry program?

2. Which sleep interventions, if any, are effective for improving sleep participation for this population?

\section{Quantitative Outcomes}

To address the quantitative research questions, each research participant engaged in a multicomponent sleep program with a baseline phase of 1 to 2 weeks and three intervention phases, which were completed in variable order for each study participant. Interventions focused 
on environmental and/or behavioral modifications to participants' routines in order to promote improved sleep. The first participant to participate in the sleep program was John.

Table 1

Participant Characteristics

$\mathrm{N}=3$

Participant One

Age

Gender

Marital Status

Diagnosis

Number of Days Post-BI to Study Initiation

Initial Insomnia Severity Index Score

Trail Making Test, Part B Score

Diagnosed Sleep Disorder

Prescribed Sleep Medication

\section{Participant Two}

Age

Gender

Marital Status

Diagnosis

Number of Days Post-BI to Study Initiation

Initial Insomnia Severity Index Score

Trail Making Test, Part B Score

Diagnosed Sleep Disorder

Prescribed Sleep Medication
50 years old

Male

Married

CVA

28

20

$69,97 \%=$ Above Average

Yes, Sleep Apnea

Trazadone, Melatonin
56 years old

Male

Married

CVA

55

17

51, 54\% = Average

Yes, Sleep Apnea

Trazadone

48 years old

Male

Married

CVA

62

12

$41,18 \%=$ Low Average

No

No 


\section{Participant One - John}

John initiated participation in the sleep program in August 2019 and completed the program 5 weeks later. When completing his sleep history forms, John indicated difficulty with falling asleep, staying asleep, worrying in bed, having inconsistent wake times, and having an uncomfortable sleep environment. He was diagnosed with obstructive sleep apnea 3 weeks into the study and was issued a continuous positive airway pressure (CPAP) machine, which he reported often not wearing due to discomfort and despite encouragement from the principal investigator. John's baseline phase for the sleep program occurred over a 2-week period. Baseline data were collected for an initial 7 days, and, when baseline stability was not achieved, an additional 7 days of baseline data were collected. However, John still did not achieve baseline stability during the 14-day baseline phase. Due to the severity of his sleep deficits, the principal investigator and the dissertation committee chair agreed to allow John to proceed to the intervention phases of the study, despite him not achieving baseline stability. For John, the study phases occurred in the order of baseline data collection, intervention phase $B$, intervention phase C, and then intervention phase D. Data were collected in all phases of the study using both the sleep diary and the Fitbit device. Sleep metrics measured in all phases included sleep onset latency, total sleep time, sleep efficiency, and number of awakenings.

\section{Sleep Onset Latency}

Sleep onset latency was measured by the time that it took to fall asleep in minutes. It is graphically depicted in Figure 7 for sleep diary data and in Figure 8 for Fitbit data.

Consensus Sleep Diary. On the sleep diary, sleep onset latency was calculated using question number three, which indicated how long it took to fall asleep each night. Figure 7 depicts sleep onset latency for all phases of the study using sleep diary data for participant one. 


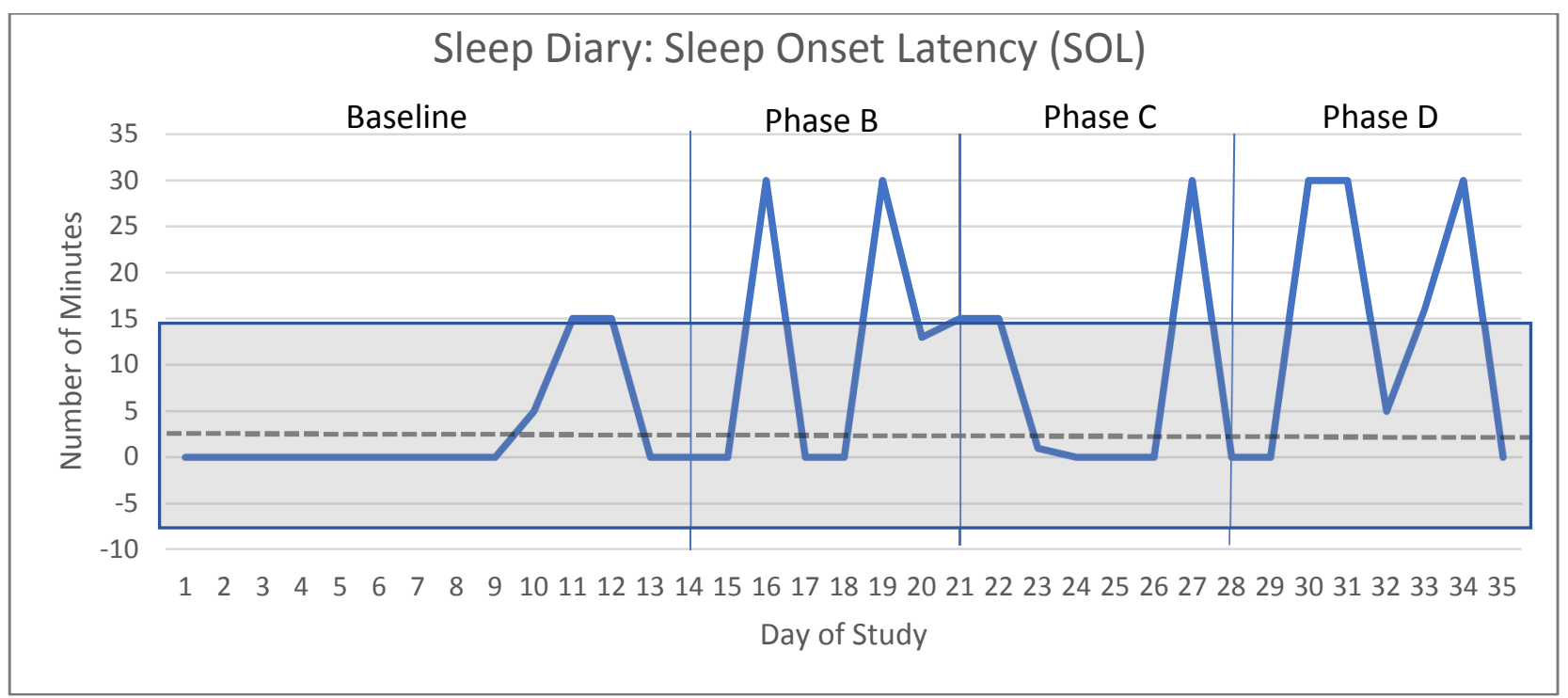

Figure 7. Participant One Sleep Diary Sleep Onset Latency. Dashed lines represent the mean of the baseline phase. The gray squared box represents the Two Standard Deviation Band.

Using visual analysis techniques of the graph in Figure 7, the level, trend, slope, and variability were calculated for sleep onset latency from the sleep diary data.

Level. Level was determined by comparing the mean values of sleep onset latency within each phase. The mean value for sleep onset latency of the baseline phase is depicted in Figure 7, which was 3.21. The mean for phase $\mathrm{B}$ was 12.57 , phase $\mathrm{C}$ was 6.57 , and phase $\mathrm{D}$ was 15.86 . This indicated that it took longer for John to fall asleep during each of the intervention phases than it did during the baseline period. During phase C, John had the lowest average amount of time to fall asleep.

Trend. Data for sleep onset latency did not show a consistent trend, indicating fluctuations in sleep onset latency throughout the study and no consistent change between baseline and intervention phases.

Variability. The direction of change was variable throughout the study phases, with the most significant variability in data noted in phases $\mathrm{B}$ and $\mathrm{D}$, indicating less stable data and less significant results in these phases. 
Inferential statistics. The two standard deviation band method was utilized to evaluate statistical significance between baseline and intervention phases in the study data. On days 30 and 31 of phase D, two consecutive data points that fell outside of the two standard deviation band, which indicated a statistical significance with an in increase in sleep onset latency.

Fitbit Inspire HR. Figure 8 depicts sleep onset latency for all phases of the study using Fitbit data for participant one.

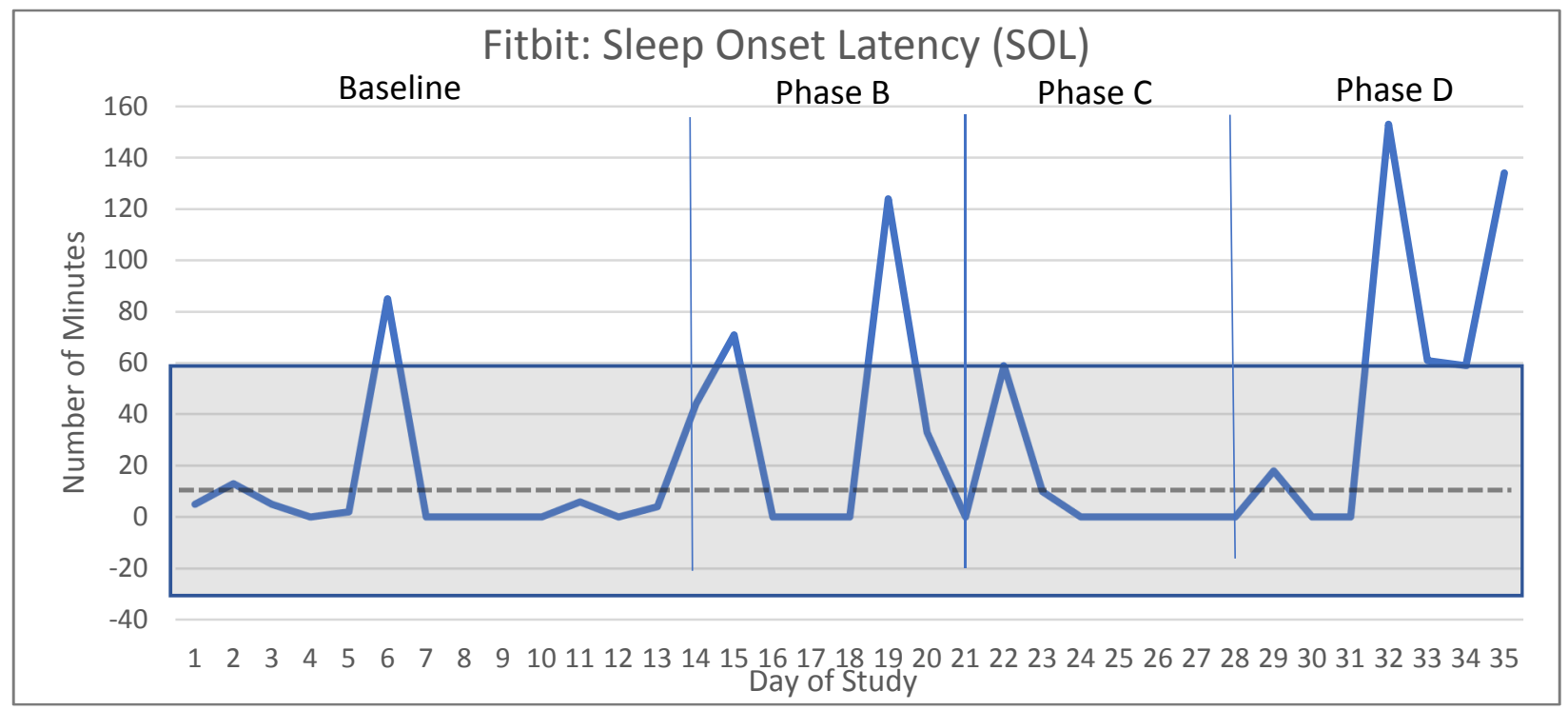

Figure 8. Participant One Fitbit Sleep Onset Latency. Dashed lines represent the mean of the baseline phase. The gray squared box represents the Two Standard Deviation Band.

Using visual analysis techniques of the graph in Figure 8, the level, trend, slope, and variability were calculated for sleep onset latency from the Fitbit data.

Level. The mean value for sleep onset latency of the baseline phase is depicted in Figure 8, which was 11.71. The mean for phase B was 32.57 , phase $\mathrm{C}$ was 9.86 , and phase $\mathrm{D}$ was 60.71 , indicating that it took longer for John to fall asleep during intervention phases B and D than it did during the baseline period. Similar to the sleep diary data, phase $\mathrm{C}$ had the lowest average amount of time to fall asleep out of all of the intervention phases, which was less than the mean for the baseline period for sleep onset latency. 
Trend. Data for sleep onset latency did not show a consistent trend across the baseline phase and phase $\mathrm{B}$, but the trend was decelerating in phase $\mathrm{C}$ and accelerating in phase $\mathrm{D}$, indicating fluctuations in sleep onset latency throughout the study. The direction of change was linear in phase $\mathrm{C}$ and curvilinear in phase $\mathrm{D}$, indicating more consistent rates of change in these phases.

Variability. The direction of change was variable throughout the baseline and intervention phases B and D but was stable in phase $\mathrm{C}$, indicating a consistent decrease in sleep onset latency that occurred in phase C.

Inferential statistics. Using the two standard deviation band method, days 32 and 33 showed a statistical significance in intervention phase D with in an increase in sleep onset latency.

Comparison of sleep diary and Fitbit data. Data were collected simultaneously throughout the study using both the Consensus Sleep Diary and the Fitbit Inspire HR for each of the sleep metrics measured in the study and in each phase of the study. Figure 9 depicts a graphical comparison of these data for sleep onset latency for participant one.

When comparing data for sleep onset latency from the sleep diary and the Fitbit, both outcome measures showed variability between the phases, with more significant variability noted in Fitbit data. Both outcome measures showed that phase $\mathrm{C}$ had the lowest mean for sleep onset latency, followed by phase $\mathrm{B}$, and then phase $\mathrm{D}$ when compared to the mean of the baseline phase. Phase $\mathrm{C}$ was the second intervention phase for John. 


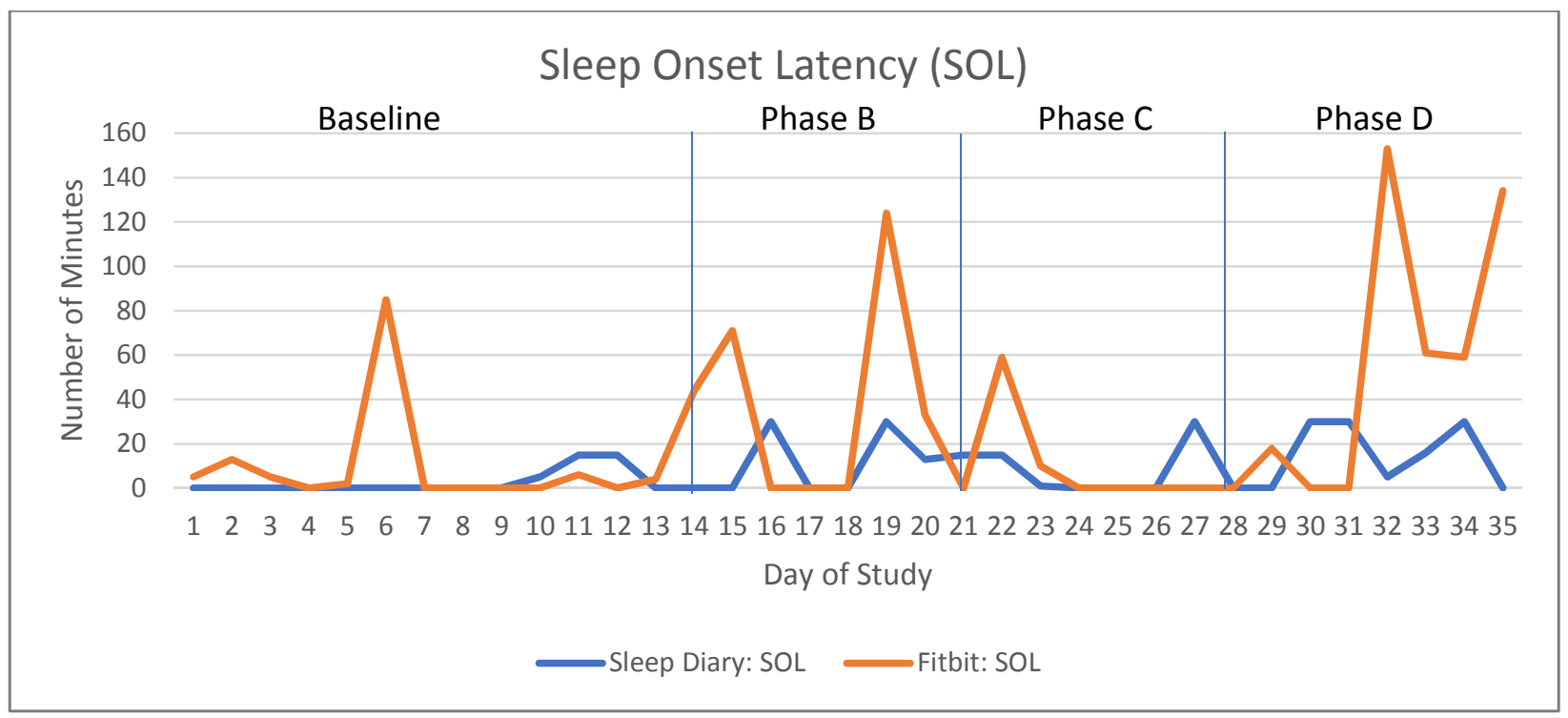

Figure 9. Participant One Combined Data Sleep Onset Latency.

\section{Total Sleep Time}

Total sleep time was measured as the amount of time spent sleeping in minutes. It is graphically depicted in Figure 10 for sleep diary data and in Figure 15 for Fitbit data.

Consensus Sleep Diary. Figure 10 depicts total sleep time for all phases of the study using sleep diary data for participant one.

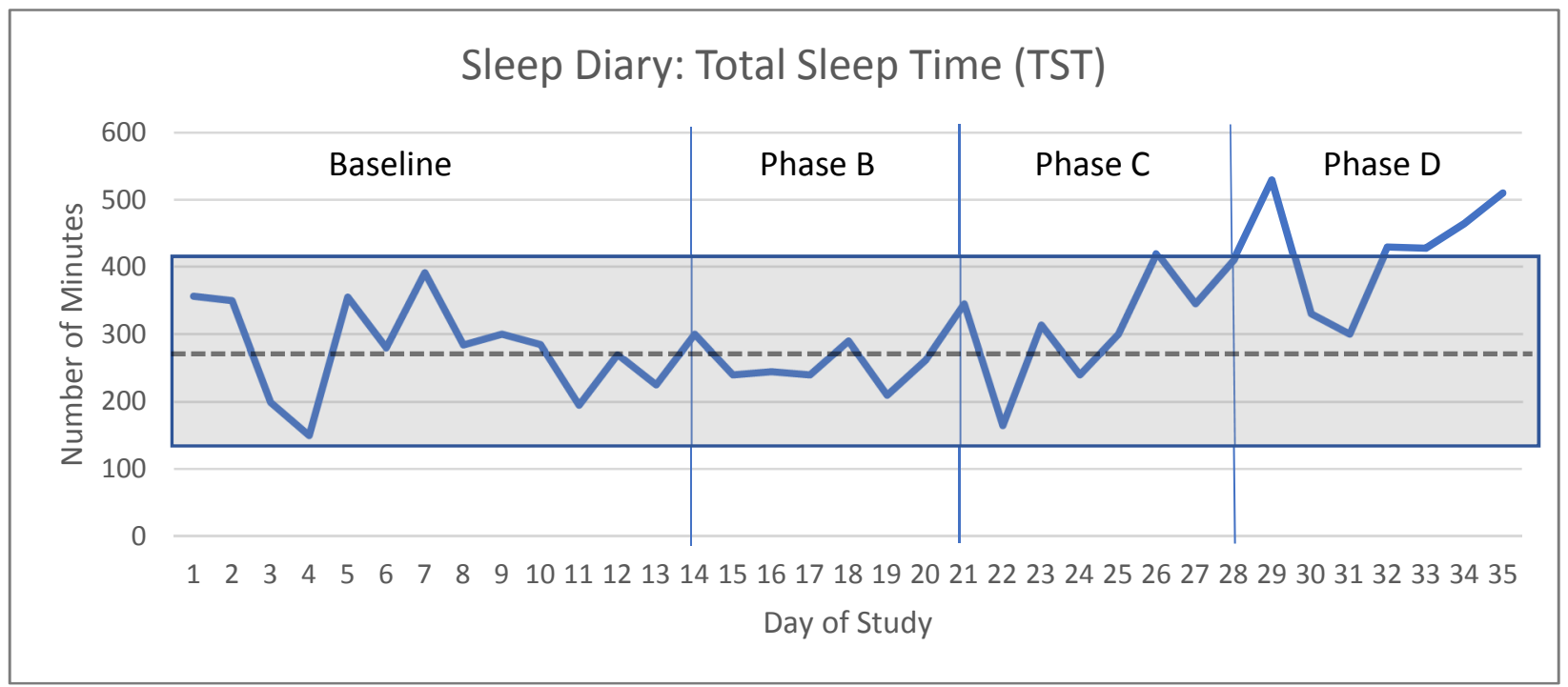

Figure 10. Participant One Sleep Diary Total Sleep Time. Dashed lines represent the mean of the baseline phase. The gray squared box represents the Two Standard Deviation Band. 
Using visual analysis techniques of the graph in Figure 10, the level, trend, slope, and variability were calculated for total sleep time from sleep diary data.

Level. The mean value for total sleep time of the baseline phase is depicted in Figure 10, which was 281.43. The mean for phase B was 261.71, phase $\mathrm{C}$ was 313.43 , and phase D was 427.57, indicating that there was an improvement in total sleep time during the last two intervention phases, with the most significant increase noted in phase D.

Trend. Data for total sleep time showed no trend in baseline data or phase B and an accelerating trend in intervention phases $\mathrm{C}$ and $\mathrm{D}$, indicating an improvement in total sleep time as the study progressed. The direction of change was linear in phase $\mathrm{C}$ and curvilinear in phase D, indicating an overall consistent increase in total sleep time in those phases. A celeration line was calculated for total sleep time using the sleep diary data and is graphically depicted in Figure 11.

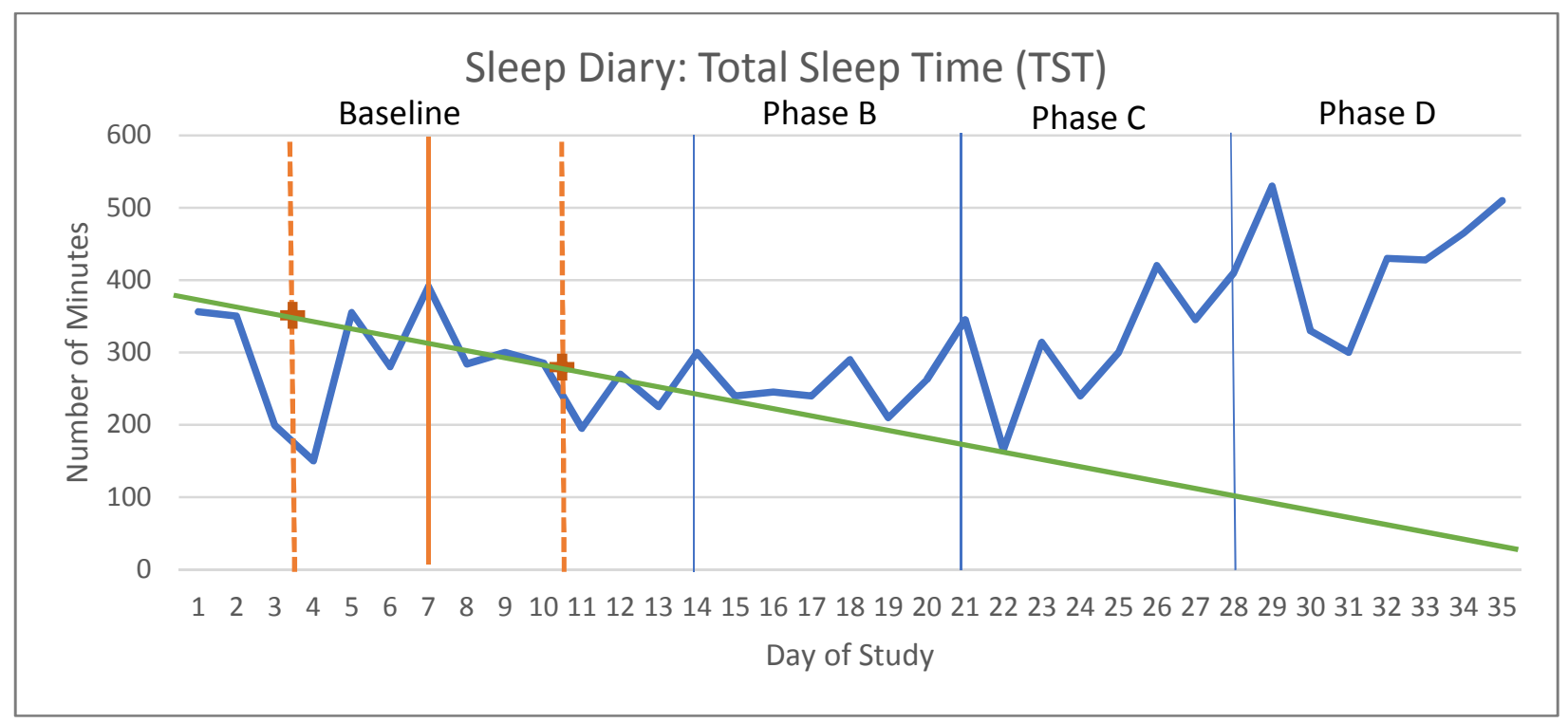

Figure 11. Participant One Total Sleep Time Celeration Line. The solid orange line represents a dividing line for half of the baseline data. Dashed orange lines represent a dividing line for quarters of the baseline data. The solid green line represents the celeration line. 
Each of the data points in all of the intervention phases fell above the celeration line, indicating a significant change in John's response pattern during the intervention phases when compared to the baseline phase.

Slope. The slope of the trend line was calculated for each of the phases to show the change in the angle of the trend. A comparison between the intervention phases was done by calculating the slope for each phase individually. The slope of data in phase B was 1.08 and decelerating, indicating that total sleep time decreased at an average rate of 1.08 times per week during this phase. The slope of data in phase $\mathrm{C}$ was 1.95 and accelerating, indicating that total sleep time increased at an average rate of 1.95 times per week during this phase. The slope of data in phase D was 1.77 and accelerating, indicating that total sleep time increased at an average rate of 1.77 times per week during this phase. The most significant rate of change was noted in intervention phase $\mathrm{C}$. The slope of total sleep time data for each intervention phase is visually depicted in Figures 12, 13, and 14.

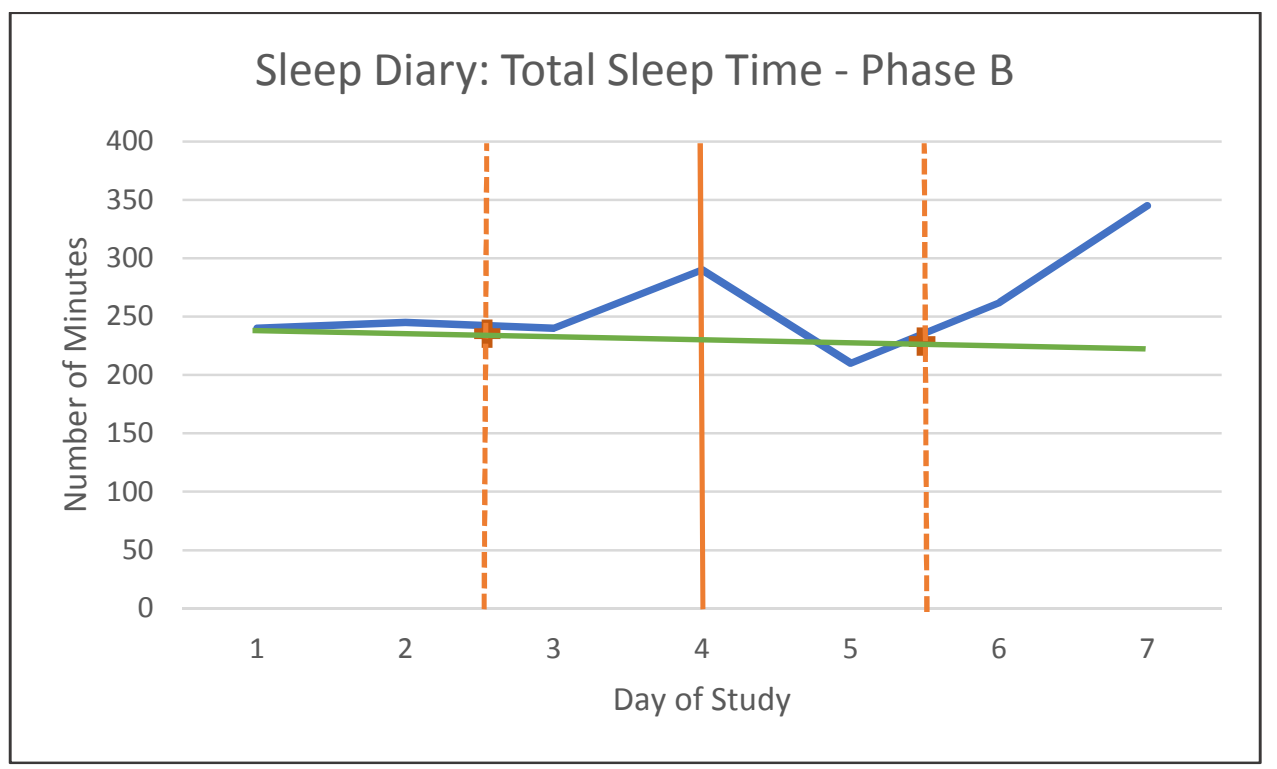

Figure 12. Participant One Sleep Diary Total Sleep Time Slope Phase B. The solid orange line represents a dividing line for half of the baseline data. Dashed orange lines represent a dividing line for quarters of the baseline data. The solid green line represents the celeration line. 


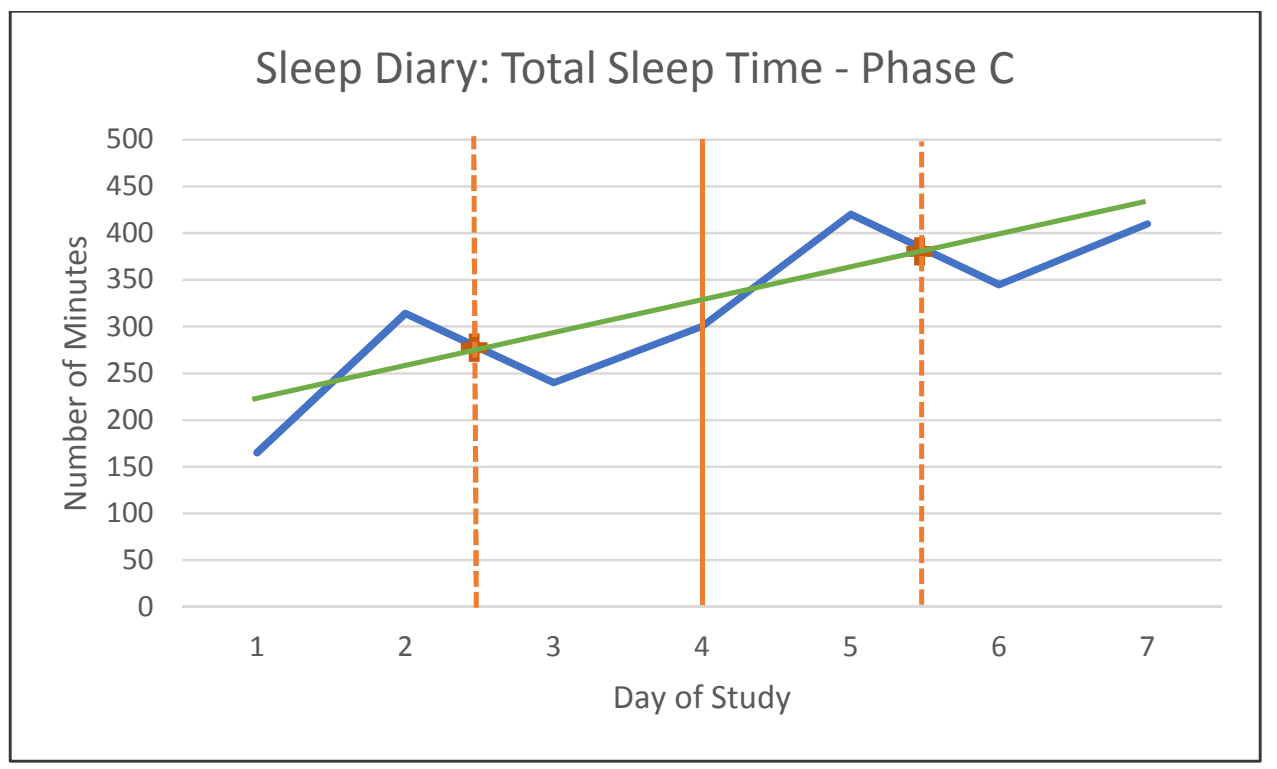

Figure 13. Participant One Sleep Diary Total Sleep Time Slope Phase C. The solid orange line represents a dividing line for half of the baseline data. Dashed orange lines represent a dividing line for quarters of the baseline data. The solid green line represents the celeration line.

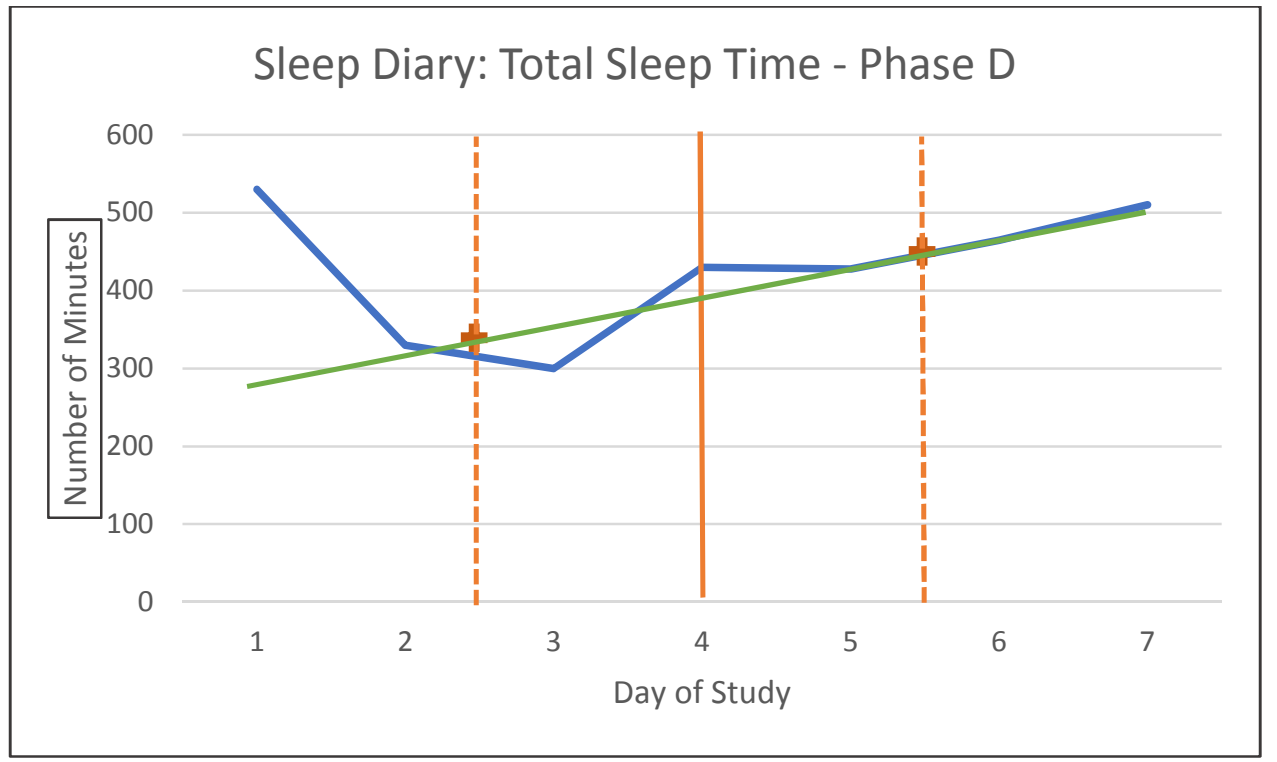

Figure 14. Participant One Sleep Diary Total Sleep Time Slope Phase D. The solid orange line represents a dividing line for half of the baseline data. Dashed orange lines represent a dividing line for quarters of the baseline data. The solid green line represents the celeration line.

Variability. The direction of change was variable throughout the study phases, with the most significant variability noted during the baseline phase. 
Inferential statistics. Using the two standard deviation band method, five out of seven data points fell outside of the two standard deviation band with statistical significance noted on days 32 through 35 of phase $\mathrm{D}$ with in an increase in total sleep time. The $\mathrm{C}$ statistic was calculated for the baseline phase, which yielded $\mathrm{C}=0.0631$ with a standard error of 0.248 . The $\mathrm{z}$ score was $0.254(0.254<1.64)$, indicating no significant trend in the baseline data. Baseline data were then combined with data from the intervention phases, yielding $\mathrm{C}=0.4807$ with a standard error of 0.1642 . The $\mathrm{z}$ score for the combined data was $2.93(2.93>1.64)$, which indicated a statistically significant trend across baseline and intervention phases.

Fitbit Inspire HR. Figure 15 depicts total sleep time for all phases of the study using Fitbit data for participant one.

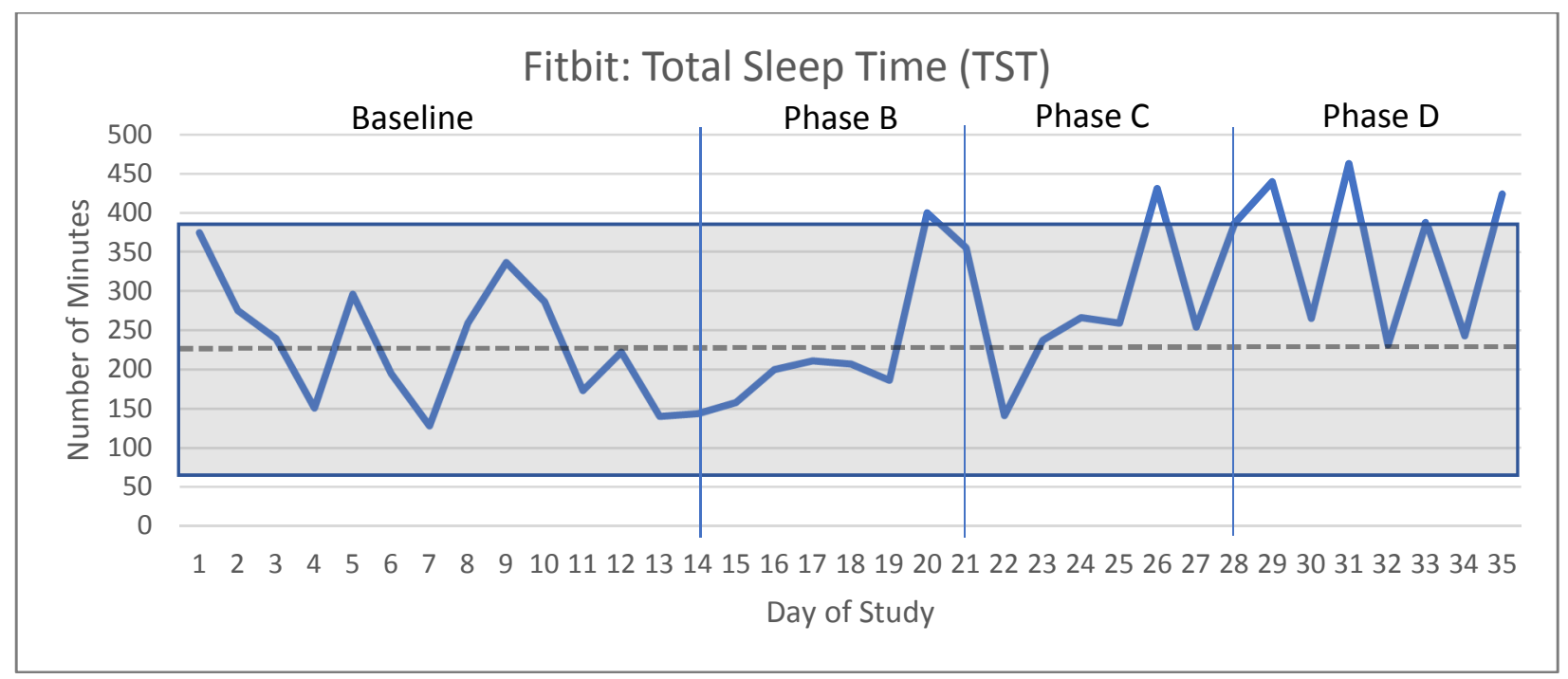

Figure 15. Participant One Fitbit Total Sleep Time. Dashed lines represent the mean of the baseline phase. The gray squared box represents the Two Standard Deviation Band.

Using visual analysis techniques of the graph in Figure 15, the level, trend, slope, and variability were calculated for total sleep time from the Fitbit data.

Level. The mean value for total sleep time of the baseline phase is depicted in Figure 15, which was 229.86. The mean for phase B was 245.29 , phase C was 282, and phase D was 
350.57 , indicating that there was a progressive improvement in total sleep time throughout the study. Similar to the sleep diary data, with the most significant increase in total sleep time was noted in phase D.

Trend. Data for total sleep time showed an accelerating trend in intervention phases B and $\mathrm{C}$ with no trend noted in phase $\mathrm{D}$. There was an overall accelerating trend during the intervention phases to the end of the study after an initial deceleration immediately after the baseline phase, indicating overall improvement in total sleep time as the study progressed. The direction of change was linear in phase B and curvilinear in phase C.

Variability. The direction of change was variable throughout the phases with only mild variability in data noted in phases $\mathrm{B}$ and $\mathrm{C}$ and significant variability noted in the baseline phase and in phase D. This indicated more consistent changes in total sleep time in phases B and C.

Inferential statistics. Using the two standard deviation band method, four of the seven data points fell outside of the two standard deviation band. However, none of these data points were consecutive, indicating no statistically significant change in total sleep time with Fitbit data.

Comparison of sleep diary and Fitbit data. Data were collected simultaneously throughout the study using both the Consensus Sleep Diary and the Fitbit Inspire HR for each of the sleep metrics measured in the study and in each phase of the study. Figure 16 depicts a graphical comparison of these data for total sleep time for participant one.

When comparing data for total sleep time from the sleep diary and the Fitbit, both outcome measures showed an accelerating trend with the most significant improvement noted in total sleep time in phase D for both. When comparing the mean of total sleep time by phase, both phases $\mathrm{C}$ and $\mathrm{D}$ showed in improvement over the mean of the baseline phase for sleep diary 
data. The Fitbit data showed an improvement in the mean of total sleep time for all phases when compared to the mean of the baseline phases. Both outcome measures yielded more than half of all data points within phase $\mathrm{D}$ above the two standard deviation band, but only data from the sleep diary showed a statistically significant improvement in total sleep time.

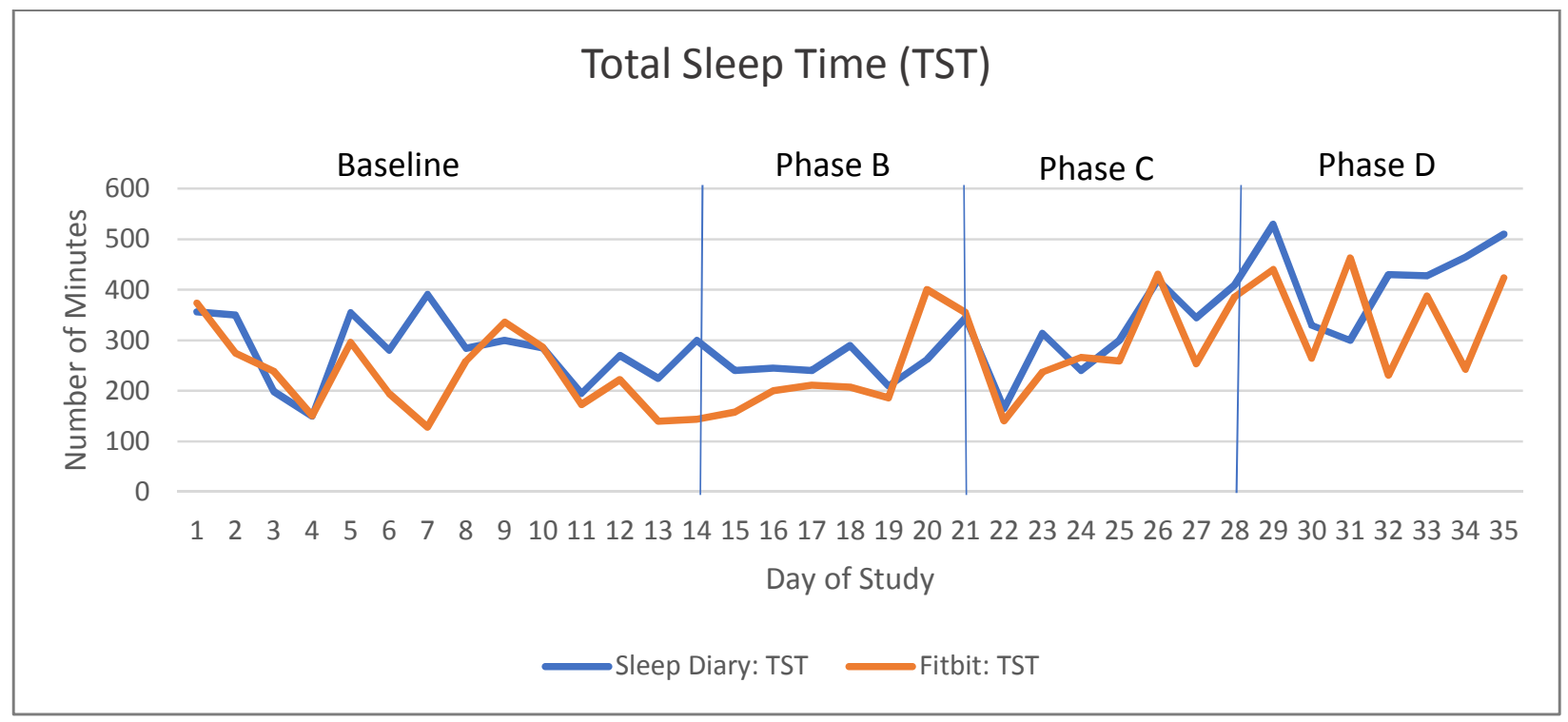

Figure 16. Participant One Combined Data Total Sleep Time.

\section{Sleep Efficiency}

Sleep efficiency is the percentage of the total time spent asleep in minutes divided by the total amount of time in bed in minutes. It is graphically depicted in Figure 17 for sleep diary data and in Figure 22 for Fitbit data.

Consensus Sleep Diary. Figure 17 depicts sleep efficiency for all phases of the study using sleep diary data for participant one. Using visual analysis techniques of the graph in Figure 17, the level, trend, slope, and variability were calculated for sleep efficiency from the sleep diary data.

Level. The mean value for sleep efficiency of the baseline phase is depicted in Figure 17, which was 70.157. The mean for phase $\mathrm{B}$ was 64.971 , phase $\mathrm{C}$ was 74.671 , and phase $\mathrm{D}$ was 
81.171, indicating that there was an improvement in sleep efficiency in the last two intervention phases compared to the baseline. The most significant improvement was in phase D.

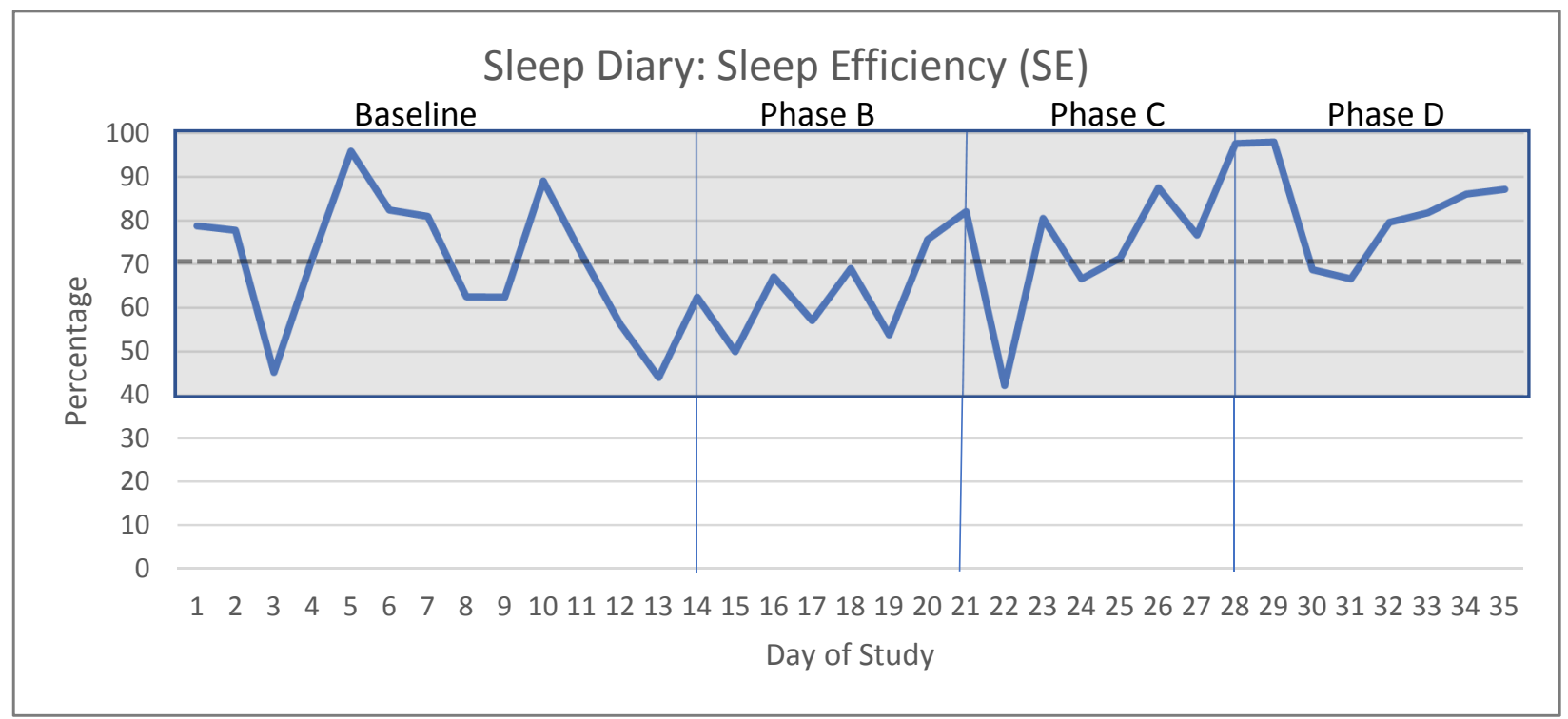

Figure 17. Participant One Sleep Diary Sleep Efficiency. Dashed lines represent the mean of the baseline phase. The gray squared box represents the Two Standard Deviation Band.

Trend. Data for sleep efficiency showed no trend for baseline data, an accelerating trend for intervention phases $\mathrm{B}$ and $\mathrm{C}$, and a brief decelerating and then accelerating trend in phase $\mathrm{D}$. The overall trend of data in the intervention phases was accelerating after an initial decline immediately after the baseline phase, indicating an overall increase in sleep efficiency as the study progressed. The direction of change was curvilinear in phase $\mathrm{D}$, indicating more consistent change in this phase. A celeration line was calculated for sleep efficiency using the sleep diary data and is graphically depicted in Figure 18. All but one of the data points in all of the intervention phases fell above the celeration line, indicating a significant change in John's response pattern during the intervention phases when compared to the baseline phase. 


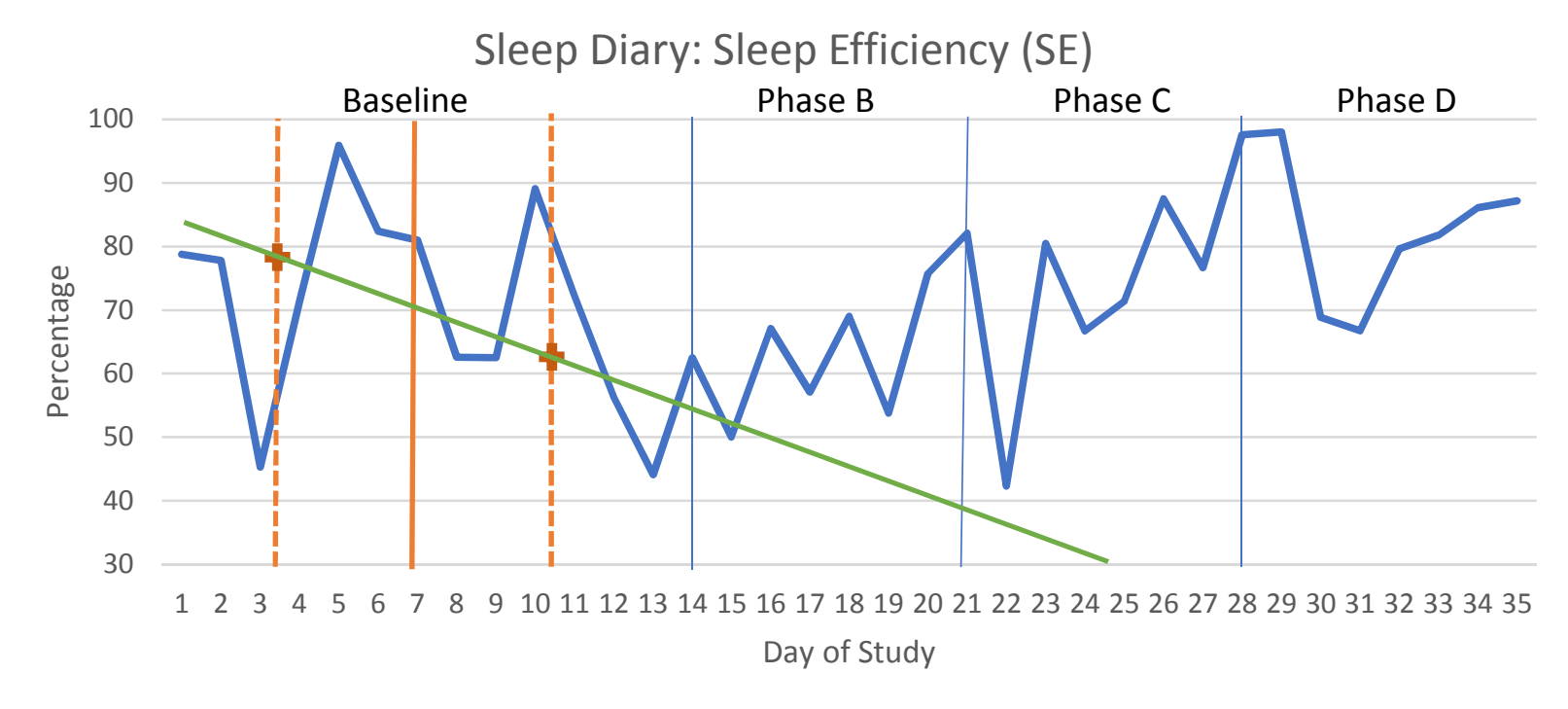

Figure 18. Participant One Sleep Diary Sleep Efficiency Celeration Line. The solid orange line represents a dividing line for half of the baseline data. Dashed orange lines represent a dividing line for quarters of the baseline data. The solid green line represents the celeration line.

Slope. The slope of data in phase B was 1.08 and accelerating, indicating that sleep efficiency increased at an average rate of 1.08 times per week during this phase. The slope of data in phase $\mathrm{C}$ was 1.21 and accelerating, indicating that sleep efficiency increased at an average rate of 1.21 times per week during this phase. The slope of data in phase D was 1.02 and accelerating, indicating that sleep efficiency increased at an average rate of 1.02 times per week during this phase. Accelerating slopes for each intervention phase indicated positive changes in sleep efficiency with the most significant rate of change noted in intervention phase C. The slope of sleep efficiency data for each intervention phase is visually depicted in Figures 19,20 , and 21. 


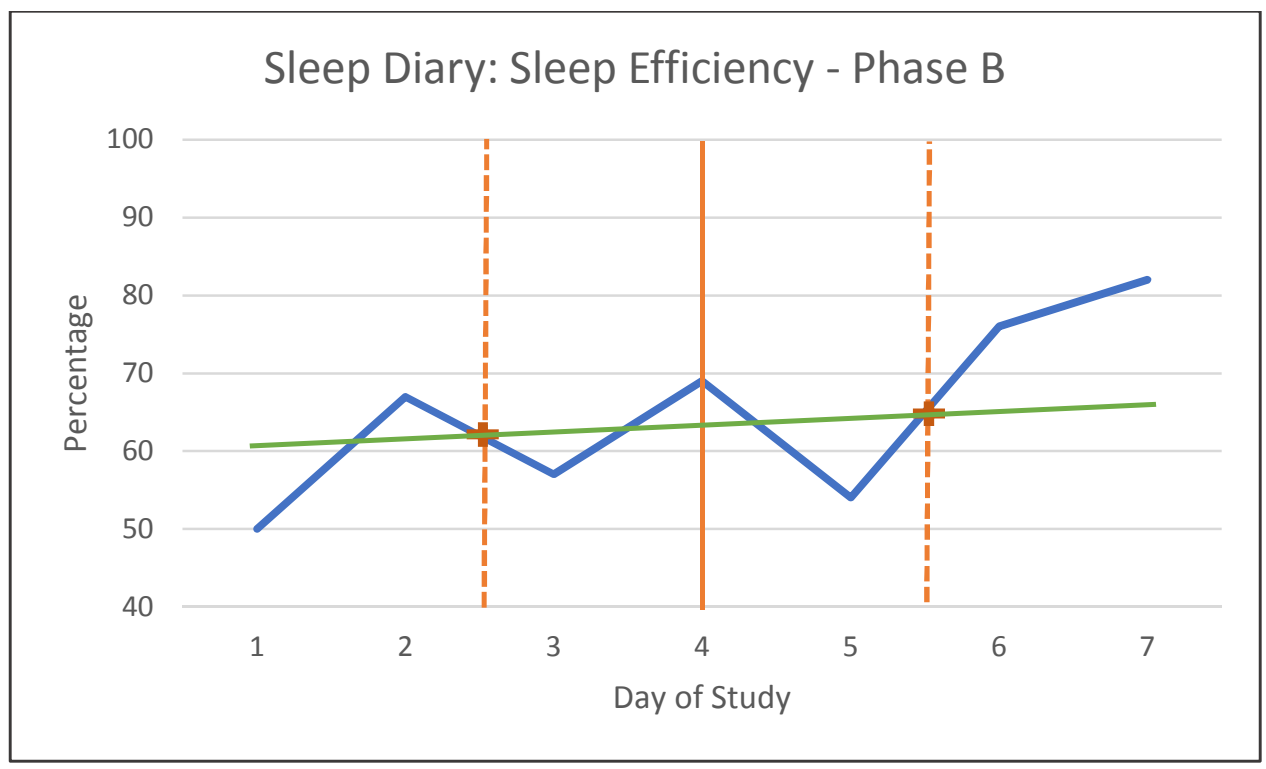

Figure 19. Participant One Sleep Diary Sleep Efficiency Slope Phase B. The solid orange line represents a dividing line for half of the baseline data. Dashed orange lines represent a dividing line for quarters of the baseline data. The solid green line represents the celeration line.

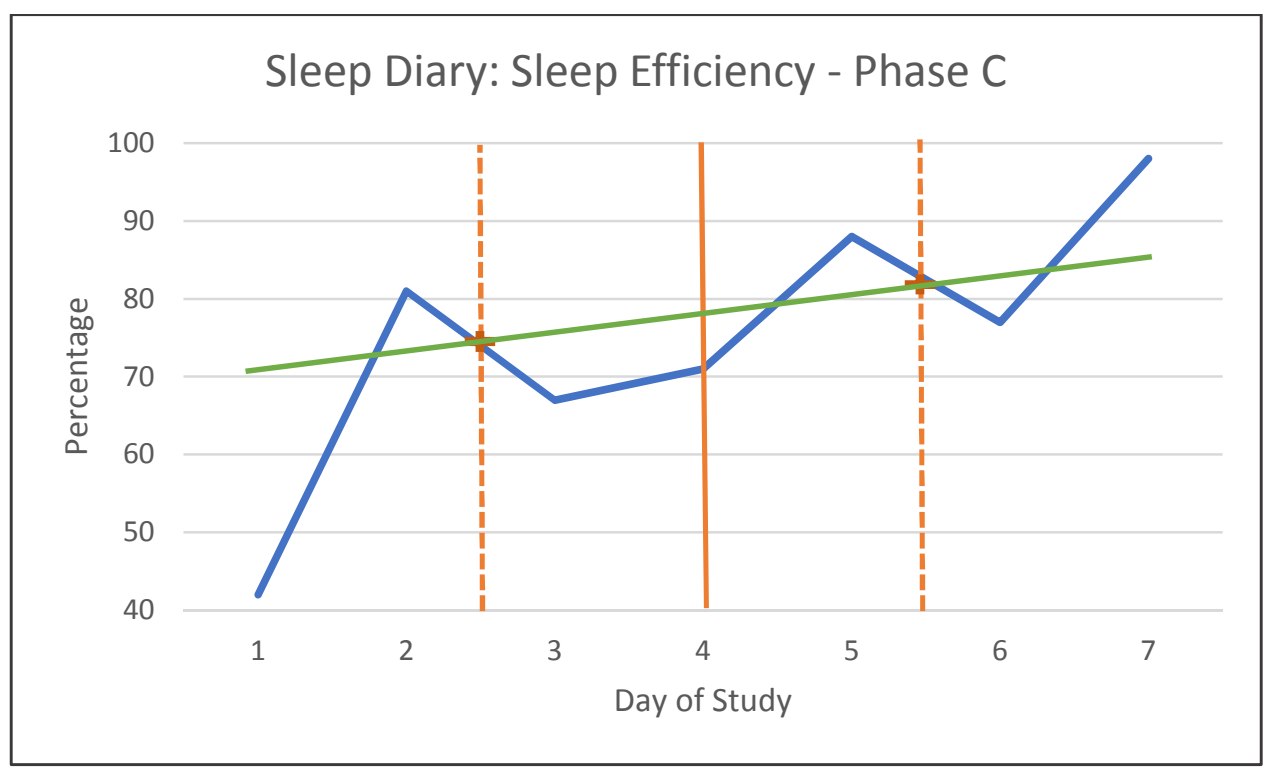

Figure 20. Participant One Sleep Diary Sleep Efficiency Slope Phase C. The solid orange line represents a dividing line for half of the baseline data. Dashed orange lines represent a dividing line for quarters of the baseline data. The solid green line represents the celeration line. 


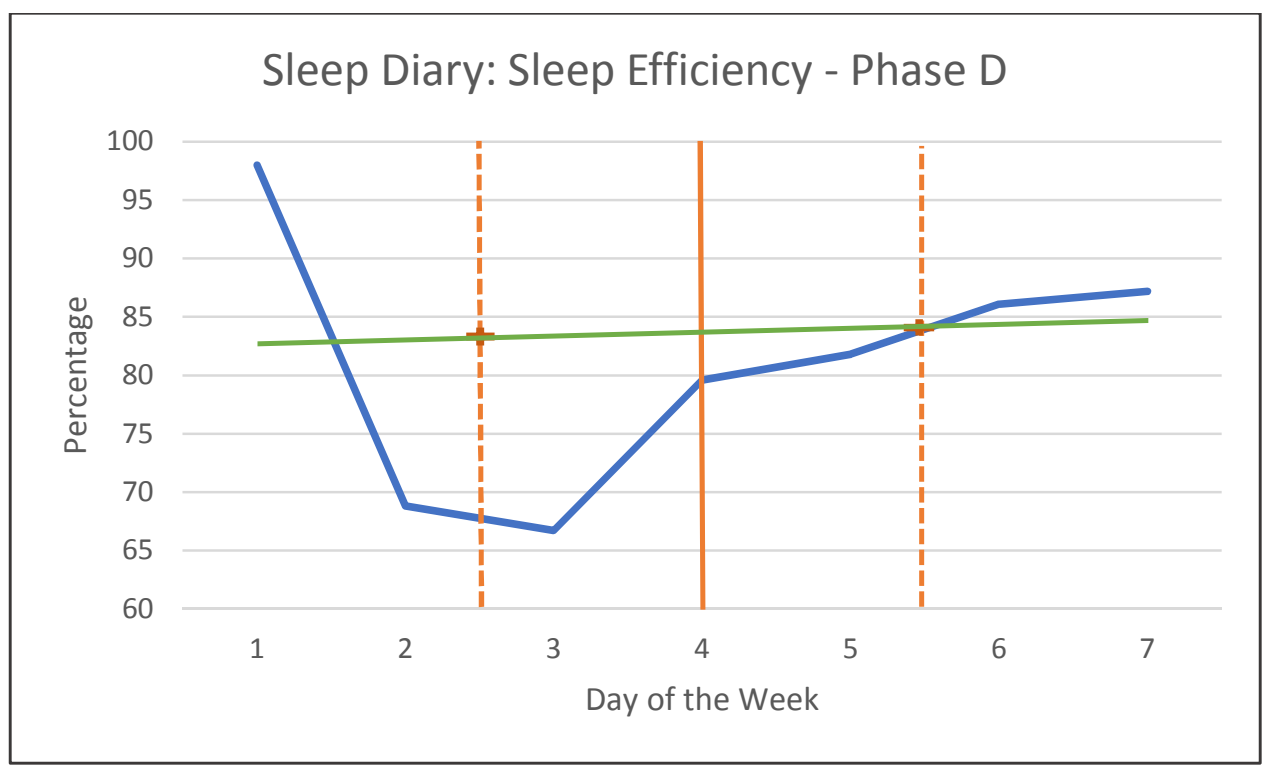

Figure 21. Participant One Sleep Diary Sleep Efficiency Slope Phase D. The solid orange line represents a dividing line for half of the baseline data. Dashed orange lines represent a dividing line for quarters of the baseline data. The solid green line represents the celeration line.

Variability. The direction of change was variable throughout the phases with less variability and more stable change noted in phase D.

Inferential statistics. Using the two standard deviation band method, none of the data points fell outside of the two standard deviation band. This indicated no statistically significant change in total sleep efficiency using sleep diary data. The $\mathrm{C}$ statistic was calculated for the baseline phase, which yielded $\mathrm{C}=0.2541$ with a standard error of 0.248 . The $\mathrm{z}$ score was 1.024 $(1.024<1.64)$, indicating no significant trend in the baseline data. Baseline data were then combined with data from the intervention phases, yielding $\mathrm{C}=0.2573$ with a standard error of 0.1642. The $\mathrm{z}$ score for the combined data was $1.56(1.56<1.64)$, which also indicated no significant trend across baseline and intervention phases.

Fitbit Inspire HR. Figure 22 depicts sleep efficiency for all phases of the study using Fitbit data for participant one. 


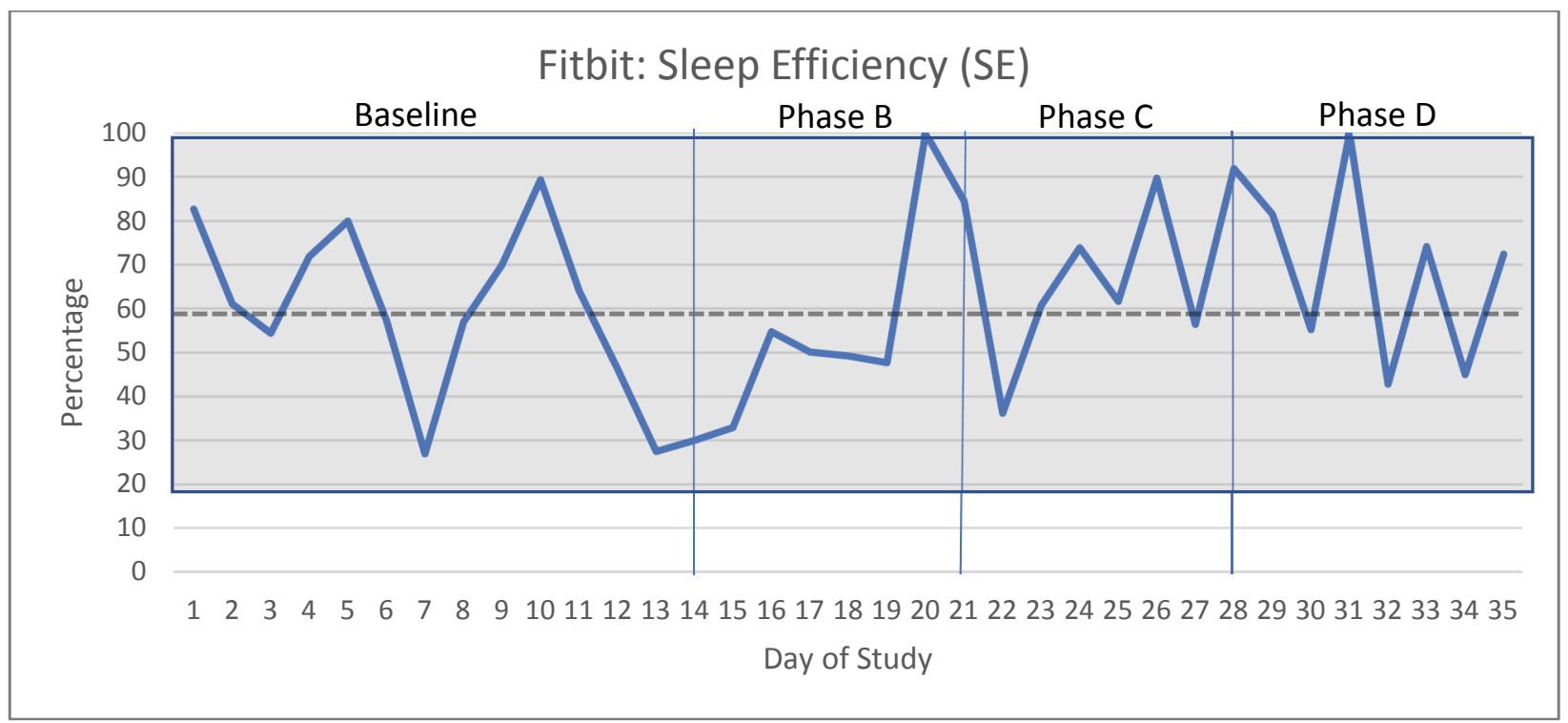

Figure 22. Participant One Fitbit Sleep Efficiency. Dashed lines represent the mean of the baseline phase. The gray squared box represents the Two Standard Deviation Band.

Using visual analysis techniques of the graph in Figure 22, the level, trend, slope, and variability were calculated for sleep efficiency from the Fitbit data.

Level. The mean value for sleep efficiency of the baseline phase is depicted in Figure 22, which was 58.457. The mean for phase B was 59.914, phase C was 67.243 , and phase D was 67.314, indicating that there was a progressive improvement in sleep efficiency throughout the intervention phases when compared to the baseline.

Trend. Data for sleep efficiency showed no trend for baseline data, an accelerating trend for intervention phases $\mathrm{B}$ and $\mathrm{C}$, and a mild decelerating trend in phase D. However, there is an overall accelerating trend in the data when comparing baseline data to data in the intervention phases, indicating a gradual increase in sleep efficiency throughout the study. The direction of change was relatively linear in phase $\mathrm{B}$, indicating more consistent change in that phase.

Variability. The direction of change was variable throughout the phases, with less variability and more stable change noted in phase B. 
Inferential statistics. Using the two standard deviation band method, only two data points in separate phases fell outside of the two standard deviation band. This indicated no statistically significant change in sleep efficiency.

Comparison of sleep diary and Fitbit data. Data were collected simultaneously throughout the study using both the Consensus Sleep Diary and the Fitbit Inspire HR for each of the sleep metrics measured in the study and in each phase of the study. Figure 23 depicts a graphical comparison of these data for sleep efficiency for participant one.

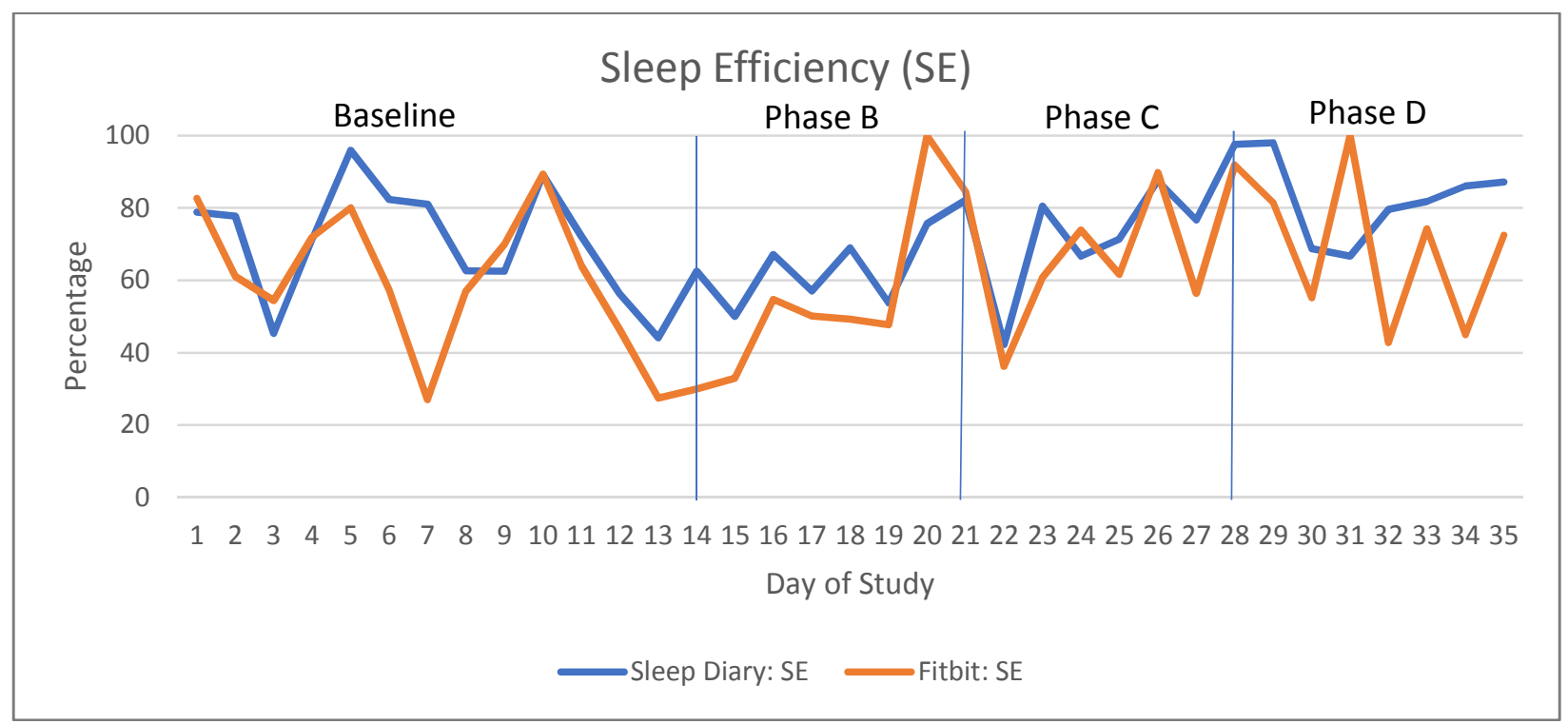

Figure 23. Participant One Combined Data Sleep Efficiency.

When comparing data for sleep efficiency from the sleep diary and the Fitbit, both outcome measures showed an initial decline in sleep efficiency immediately after the baseline period and into the first baseline phase but then an accelerating trend. The most significant improvement noted in sleep efficiency was in phase D for both the sleep diary and the Fitbit. When comparing the mean of sleep efficiency by phase, both phases C and D showed in improvement over the mean of the baseline phase for sleep diary data. The Fitbit data showed an improvement of the mean of sleep efficiency for all phases when compared to the mean of the baseline phase. 


\section{Number of Awakenings}

The number of awakenings is calculated by the number of times a person goes from a state of sleep to a state of wakefulness. It graphically depicted in Figure 24 for sleep diary data and in Figure 25 for Fitbit data.

Consensus Sleep Diary. Figure 24 depicts the number of awakenings for all phases of the study using sleep diary data for participant one.

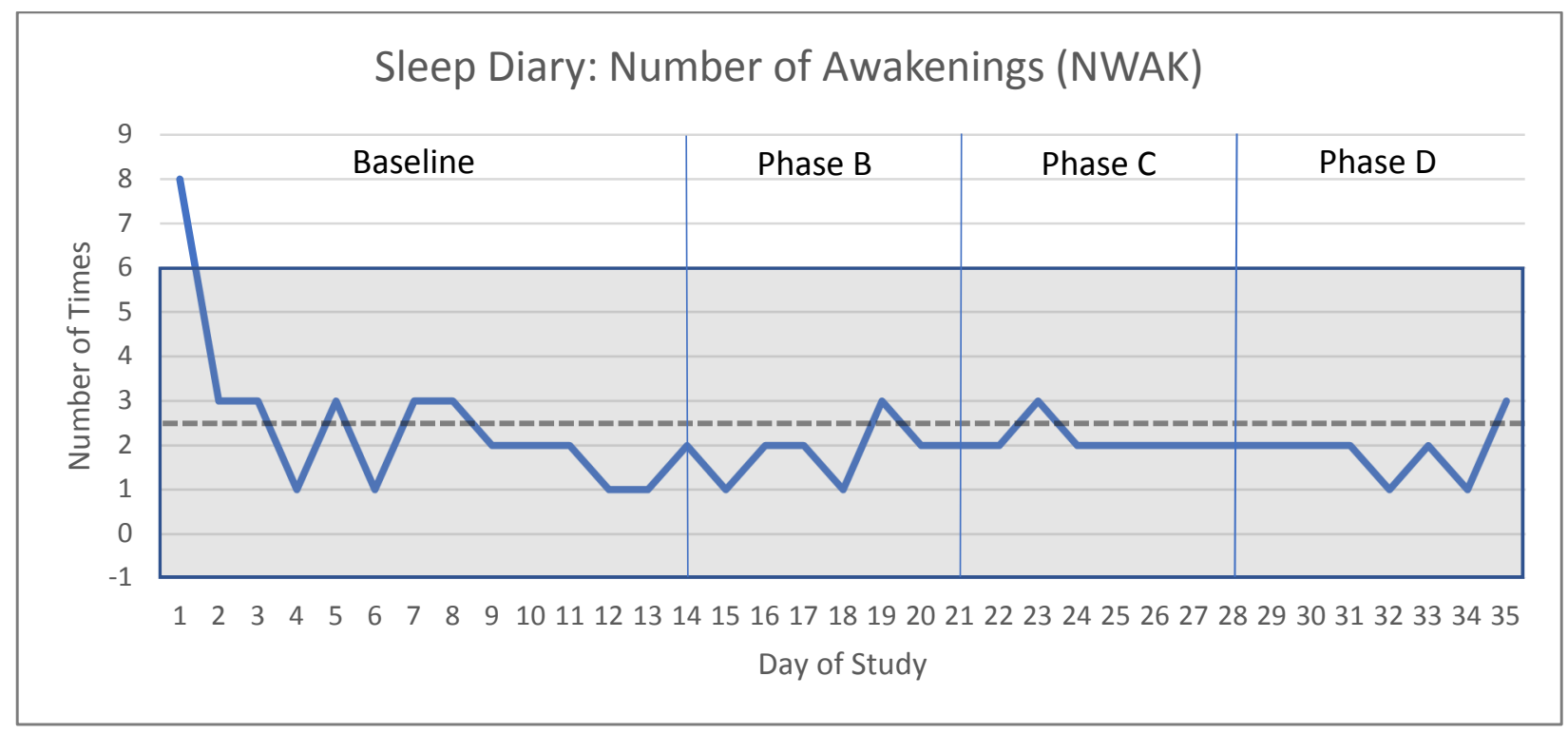

Figure 24. Participant One Sleep Diary Number of Awakenings. Dashed lines represent the mean of the baseline phase. The gray squared box represents the Two Standard Deviation Band.

Using visual analysis techniques of the graph in Figure 24, the level, trend, slope, and variability were calculated for number of awakenings from the sleep diary data.

Level. The mean value for the number of awakenings in the baseline phase is depicted in Figure 24, which was 2.50. The mean for phase B was 1.86 , phase $\mathrm{C}$ was 2.14 , and phase D was 1.86, indicating that there was a decrease in the mean number of awakenings in each of the intervention phases when compared to the baseline. The most improvement was noted in phases $\mathrm{B}$ and D. 
Trend. Data for the number of awakenings showed a mild decelerating trend for baseline data and no identifiable trend for the intervention phases, indicating no significant change in the number of awakenings throughout the study.

Variability. The direction of change was variable throughout the phases with only mild variability noted in phase $\mathrm{C}$, indicating more stable data in that phase.

Inferential statistics. Using the two standard deviation band method, no data points during the intervention phases fell outside of the two standard deviation band. This indicated no statistically significant change in the number of awakenings using the sleep diary.

Fitbit Inspire HR. Figure 25 depicts the number of awakenings for all phases of the study using Fitbit data for participant one.

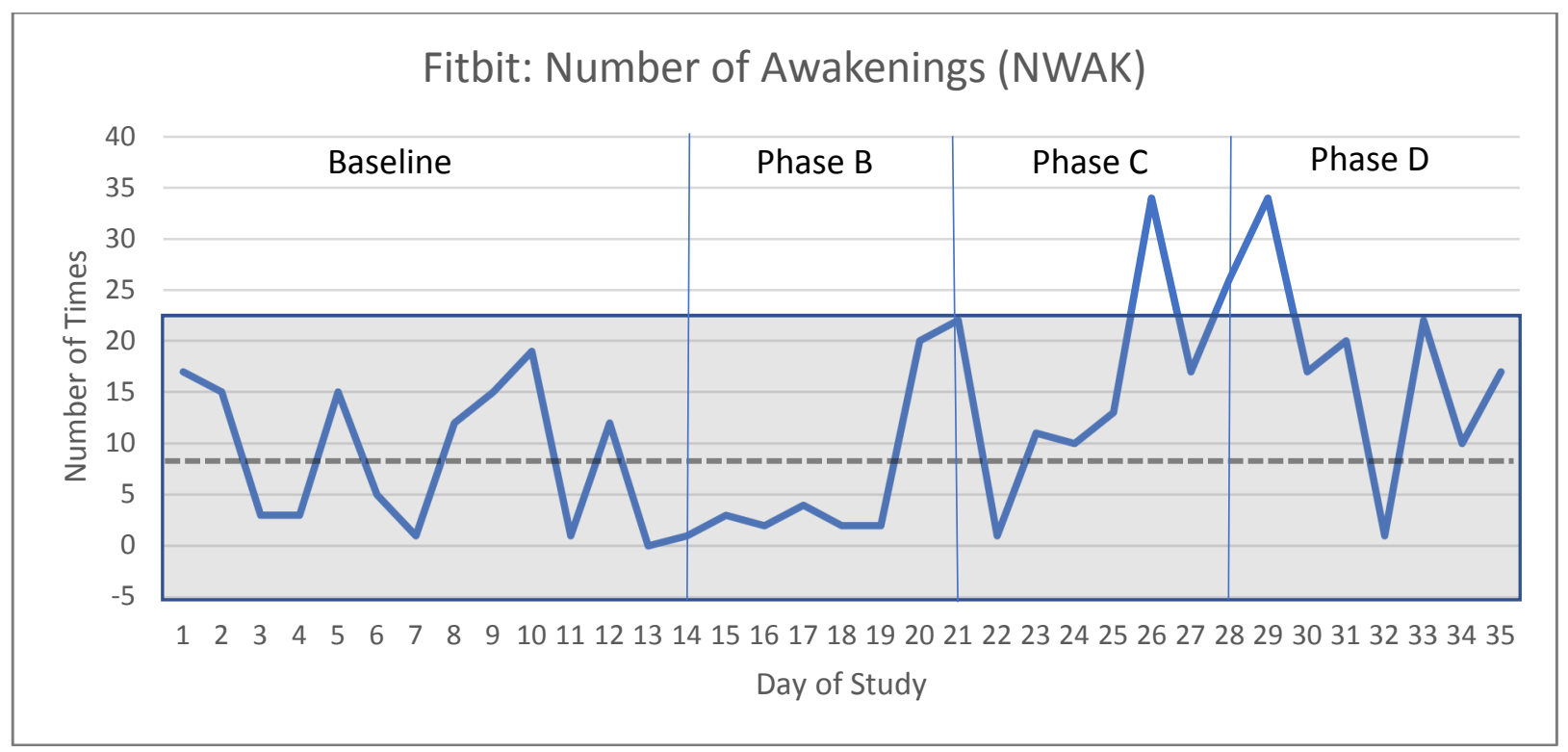

Figure 25. Participant One Fitbit Number of Awakenings. Dashed lines represent the mean of the baseline phase. The gray squared box represents the Two Standard Deviation Band.

Using visual analysis techniques of the graph in Figure 25, the level, trend, slope, and variability were calculated for the number of awakenings from the Fitbit data. 
Level. The mean value for the number of awakenings in the baseline phase is depicted in Figure 25, which was 8.50. The mean for phase B was 7.86, phase C was 16.0 , and phase D was 17.29, indicating that there was a decrease in the mean number of awakenings in phase B only when compared to the baseline.

Trend. Data for the number of awakenings showed no trend for baseline data, an accelerating trend for intervention phases $\mathrm{B}$ and $\mathrm{C}$, and a decelerating trend in phase $\mathrm{D}$, indicating fluctuations in the number of awakenings during the study. The direction of change was relatively linear in phase B, indicating more consistent changes in phase B.

Variability. The direction of change was variable throughout with less variability noted in phases $\mathrm{B}$ and $\mathrm{C}$, indicating more stable data in those phases.

Inferential statistics. Using the two standard deviation band method, no consecutive data points fell outside of the two standard deviation band. This indicated no statistically significant change in the number of awakenings.

Comparison of sleep diary and Fitbit data. Data were collected simultaneously throughout the study using both the Consensus Sleep Diary and the Fitbit Inspire HR for each of the sleep metrics measured in the study and in each phase of the study. Figure 26 depicts a graphical comparison of these data for the number of awakenings for participant one. 


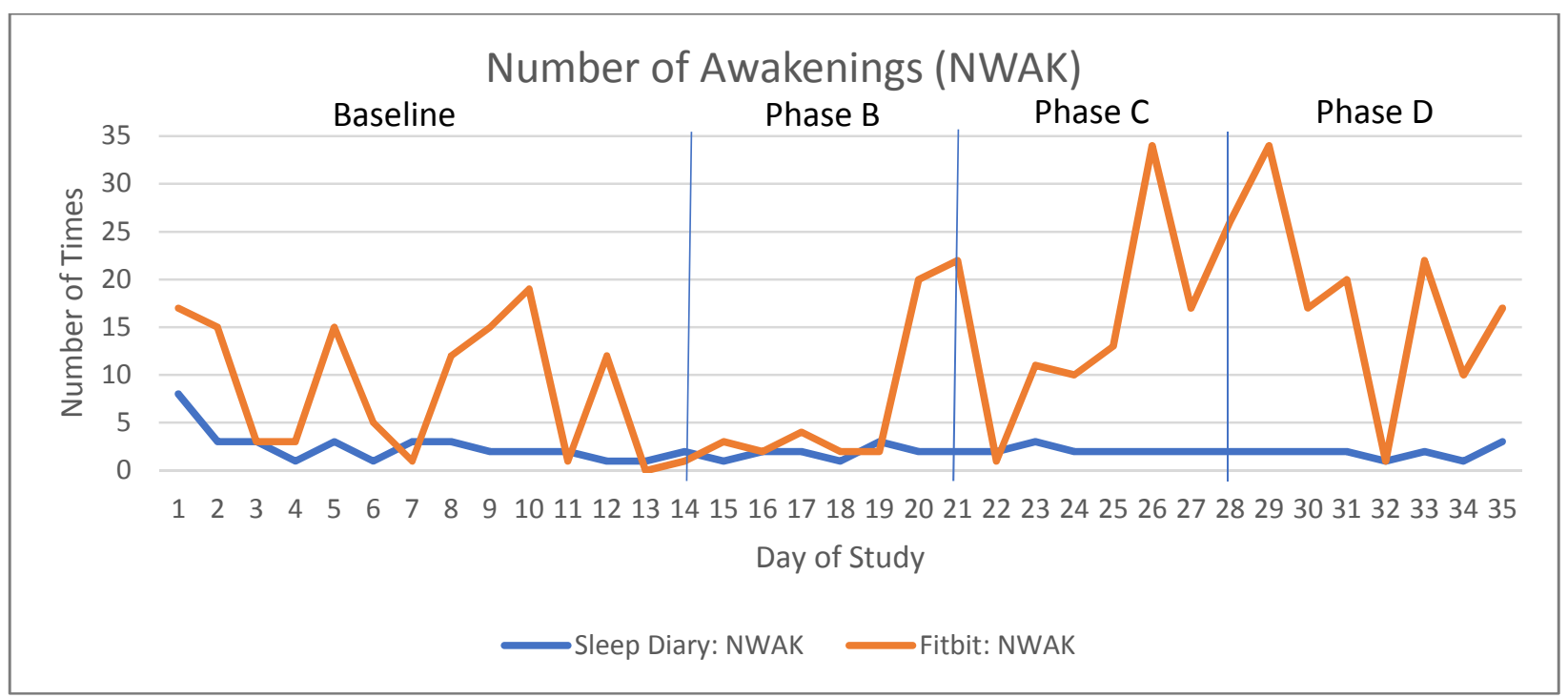

Figure 26. Participant One Combined Data Number of Awakenings.

When comparing data for the number of awakenings from the sleep diary and the Fitbit, there was no consistent trend between the two outcome measures except with the lowest number of awakenings noted in phase B for both the sleep diary and the Fitbit device. See Table 2 for a comparison of the means of each phase for all variables for the sleep diary and the Fitbit device for participant one. 
Table 2

Participant One Mean Comparison

\begin{tabular}{|c|c|c|c|c|c|c|c|c|c|c|c|c|c|c|c|c|}
\hline & \multicolumn{3}{|c|}{ Sleep Onset Latency } & \multicolumn{4}{|c|}{ Total Sleep Time } & \multicolumn{4}{|c|}{ Sleep Efficiency } & \multicolumn{5}{|c|}{ Number of Awakenings } \\
\hline & Baseline & Phase B & Phase C & Phase D & Baseline & Phase B & Phase C & Phase D & Baseline & Phase B & Phase C & Phase D & Baseline & Phase B & Phase C & Phase D \\
\hline Diary & 3.21 & 12.57 & 6.57 & 15.86 & 281.43 & 261.71 & 313.43 & 427.57 & 70.157 & 64.971 & 74.671 & 81.171 & 2.50 & 1.86 & 2.14 & 1.86 \\
\hline Fitbit & 11.71 & 32.57 & 9.86 & 60.71 & 229.86 & 245.29 & 282.00 & 350.57 & 58.457 & 59.914 & 67.243 & 67.314 & 8.50 & 7.86 & 16.00 & 17.29 \\
\hline
\end{tabular}




\section{Severity of Insomnia}

The level of insomnia was measured at the beginning of the study, after all interventions were completed, and at a one-month follow-up after interventions were completed. The severity of insomnia was measured using the Insomnia Severity Index, with lower scores indicating less insomnia. Figure 27 shows the Insomnia Severity Index assessment results for participant one.

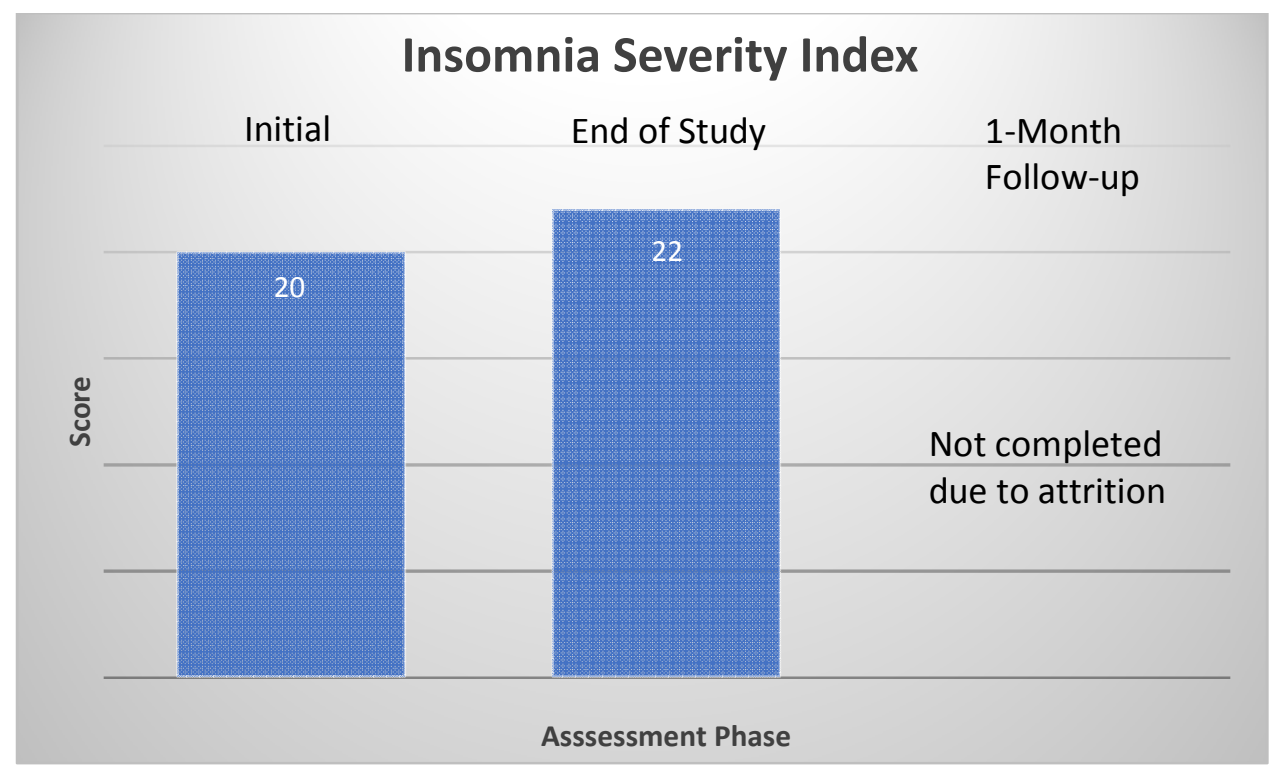

Figure 27. Participant One Insomnia Severity Index.

A total score on the Insomnia Severity Index of 15 to 21 indicates "moderate insomnia," and a score of 22 to 28 indicates "severe clinical insomnia" (Morin, 1993). The results from John's Insomnia Severity Index assessments showed that his initial score fell within the moderate insomnia range and his second score fell within the severe range. John's score increased at the end of the study when compared to his initial Insomnia Severity Index score, indicating that the severity of his insomnia increased by the end of the study. John was also unable to complete the Insomnia Severity Index at the one-month follow-up due to participant attrition. 


\section{Functional Outcomes of Sleep}

To determine the functional implications of disruptions in sleep after BI, the Functional Outcomes of Sleep Questionnaire was administered at the beginning of the study and after the completion of each intervention phase. Higher scores indicated less disruption in functional outcomes. Figure 28 shows the results of the Functional Outcomes of Sleep Questionnaire assessments for participant one.

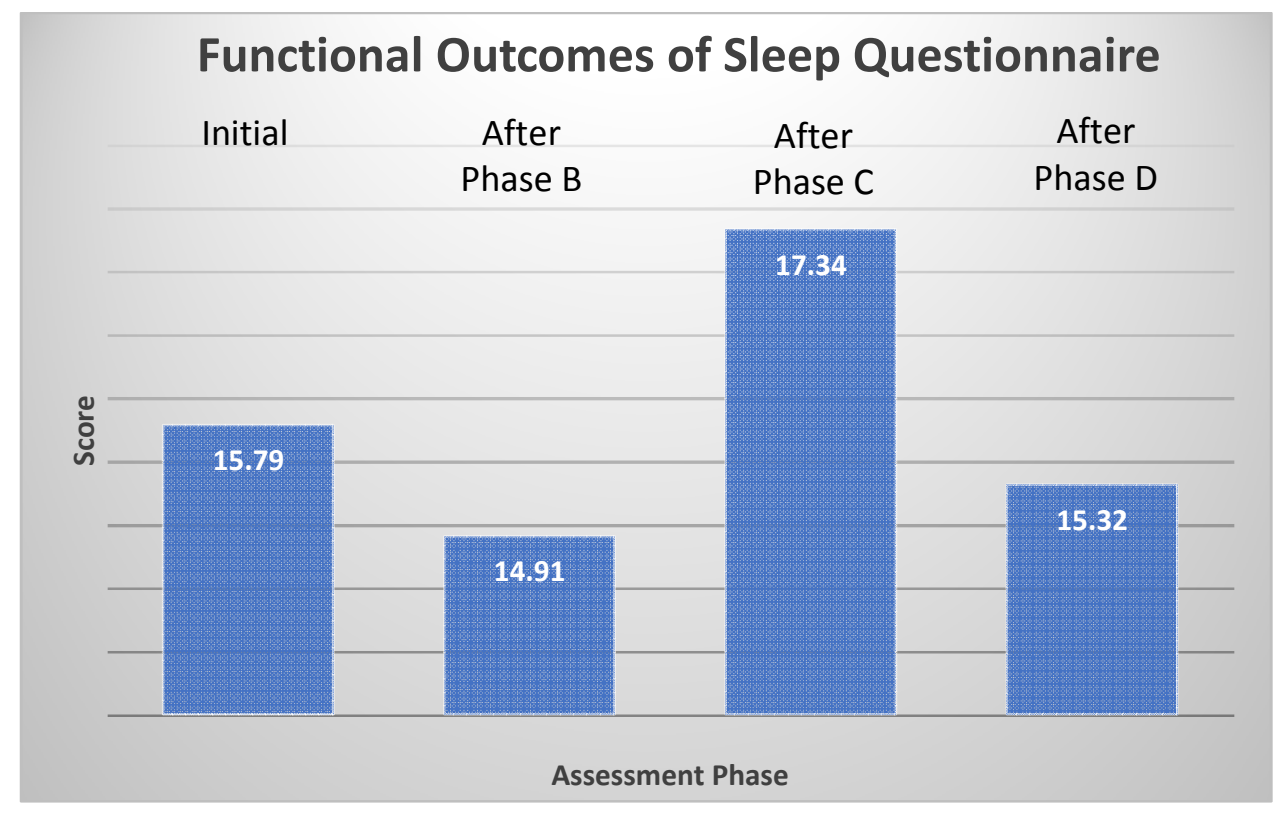

Figure 28. Participant One Functional Outcomes of Sleep Questionnaire.

The results from John's Functional Outcomes of Sleep Questionnaire assessments indicated a fluctuation in disruption of functional outcomes throughout the study. He had an improvement in functional outcomes after intervention phase $\mathrm{C}$, but an increase in functional disruption was noted after participating in phases B and D, with phase B being the most disruptive to John's functional outcomes. 


\section{Sleepiness}

To determine the level of sleepiness of participants while participating in the dissertation study, the Epworth Sleepiness Scale was administered at the beginning of the study and after the completion of each phase of the study. Lower scores indicated less sleepiness. Figure 29 shows the results of the Epworth Sleepiness Scale assessments for participant one.

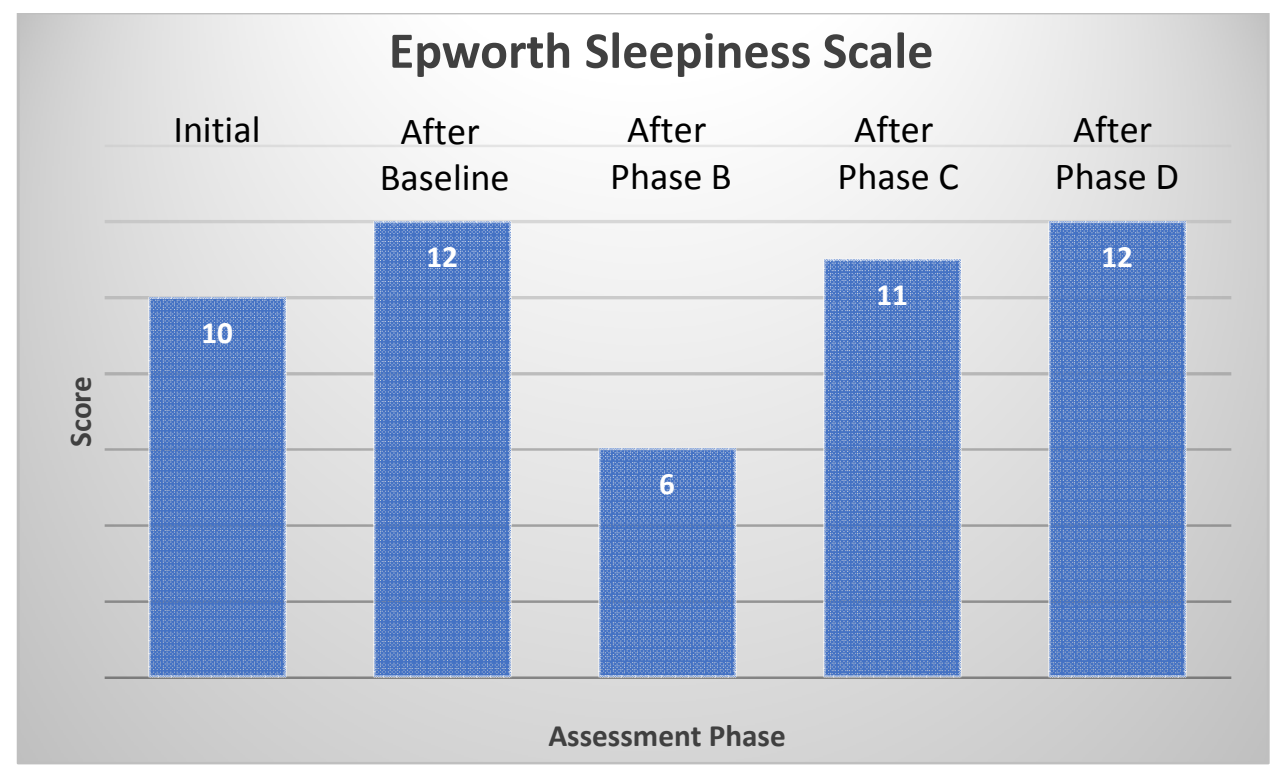

Figure 29. Participant One Epworth Sleepiness Scale.

The results of John's Epworth Sleepiness Scale assessments indicated a fluctuation in sleepiness while participating in the study. His initial Epworth Sleepiness Scale score of 10 indicated "higher normal daytime sleepiness" (Johns, 1991), and his scores after the baseline phase and after intervention phases C and D indicated "mild excessive daytime sleepiness" (Johns, 1991). His score after intervention phase B indicated "lower normal daytime sleepiness" (Johns, 1991), indicating less sleepiness after participating in this phase when compared to the rest of the study.

\section{Participant Two - Daniel}

Daniel initiated participation in the sleep program in October 2019 and completed the program 4 weeks later. When completing his sleep history forms, Daniel indicated difficulty 
with falling asleep, staying asleep, and worrying in bed. He had also been previously diagnosed with obstructive sleep apnea and had been using a CPAP machine for many years, which he consistently utilized during the dissertation study. Baseline data for the sleep program were collected for 7 days, and baseline stability was achieved during this time. For Daniel, the study phases occurred in the order of baseline data collection, intervention phase $\mathrm{C}$, intervention phase $\mathrm{D}$, and then intervention phase B. Data were collected in all phases of the study using both the sleep diary and the Fitbit device. Sleep metrics measured in all phases included sleep onset latency, total sleep time, sleep efficiency, and number of awakenings.

\section{Sleep Onset Latency}

Sleep onset latency is graphically depicted in Figure 30 for sleep diary data and in Figure 31 for Fitbit data.

Consensus Sleep Diary. Figure 30 depicts sleep onset latency for all phases of the study using sleep diary data for participant two.

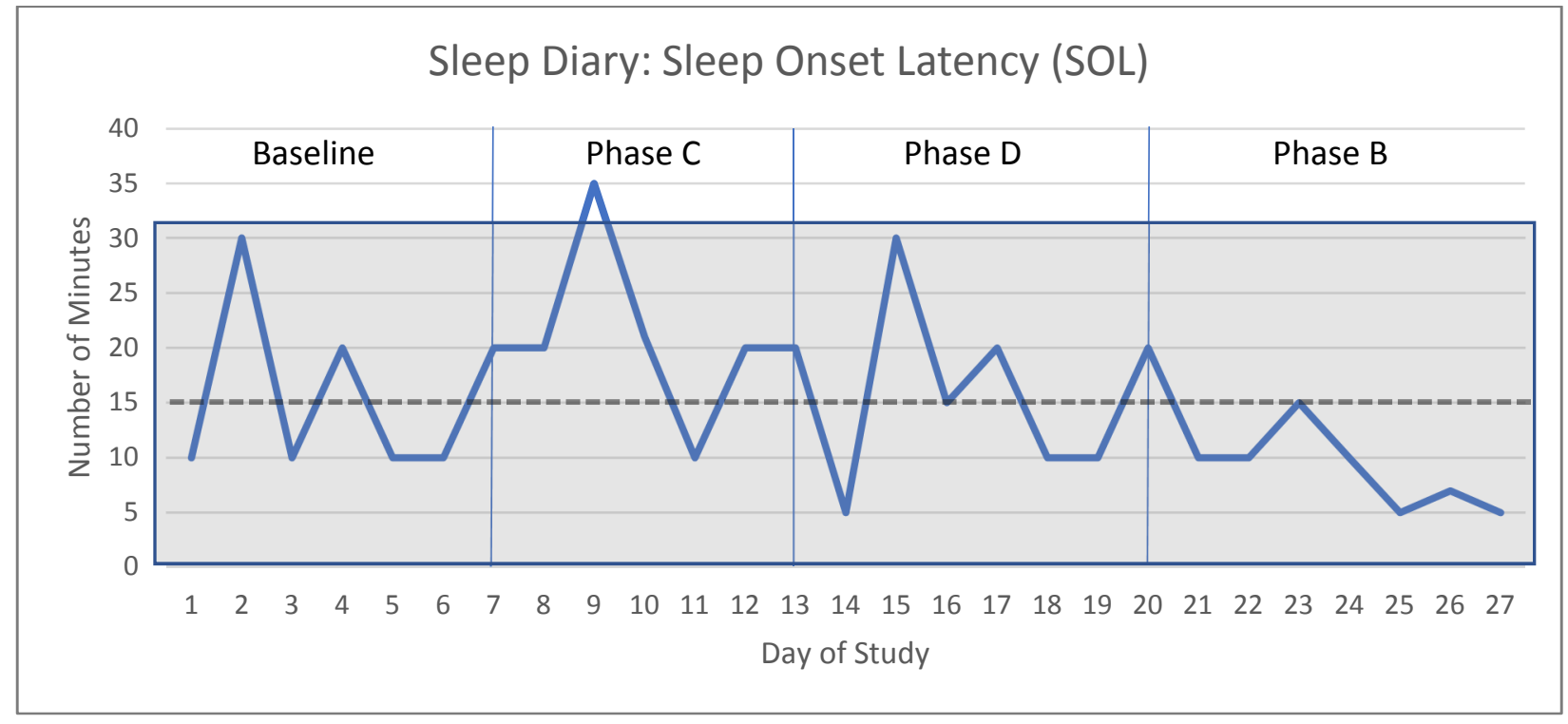

Figure 30. Participant Two Sleep Diary Sleep Onset Latency. Dashed lines represent the mean of the baseline phase. The gray squared box represents the Two Standard Deviation Band. 
Using visual analysis techniques of the graph in Figure 30, the level, trend, slope, and variability were calculated for sleep onset latency from the sleep diary data.

Level. Level was determined by comparing the mean values of sleep onset latency within each phase. The mean value for sleep onset latency of the baseline phase is depicted in Figure 30, which was 15.71. The mean for phase C was 21.0, phase D was 15.71 , and for phase B 8.86. This indicated that the average time for sleep onset latency increased for the first intervention phase, was the same for the second intervention phase, and decreased for the last intervention phase.

Trend. Data for sleep onset latency showed no trend for baseline data, a decelerating trend for all intervention phases, indicating an overall decline in sleep onset latency as the study progressed. The direction of change was curvilinear in phase B, indicating more consistent changes in sleep onset latency in phase B.

Variability. The direction of change was variable throughout the phases with less variability noted in phase $\mathrm{B}$, indicating more stable change in that phase.

Inferential statistics. Using the two standard deviation band method, only one data point fell outside of the two standard deviation band. This indicated no statistically significant change in sleep onset latency.

Fitbit Inspire HR. Figure 31 depicts sleep onset latency for all phases of the study using Fitbit data for participant two. Using visual analysis techniques of the graph in Figure 31, the level, trend, slope, and variability were calculated for sleep onset latency from the Fitbit data.

Level. The mean value for sleep onset latency of the baseline phase is depicted in Figure 31, which was 4.14. The mean for phase C was 1.17 , phase D was 19.86 , and phase B was 9.86 . This indicated that the average time for sleep onset latency decreased for the first intervention 
phase during phase $\mathrm{C}$ and increased for the last two intervention phases. The most significant increase in sleep onset latency was in phase D, with a spike on the first day of the intervention phase.

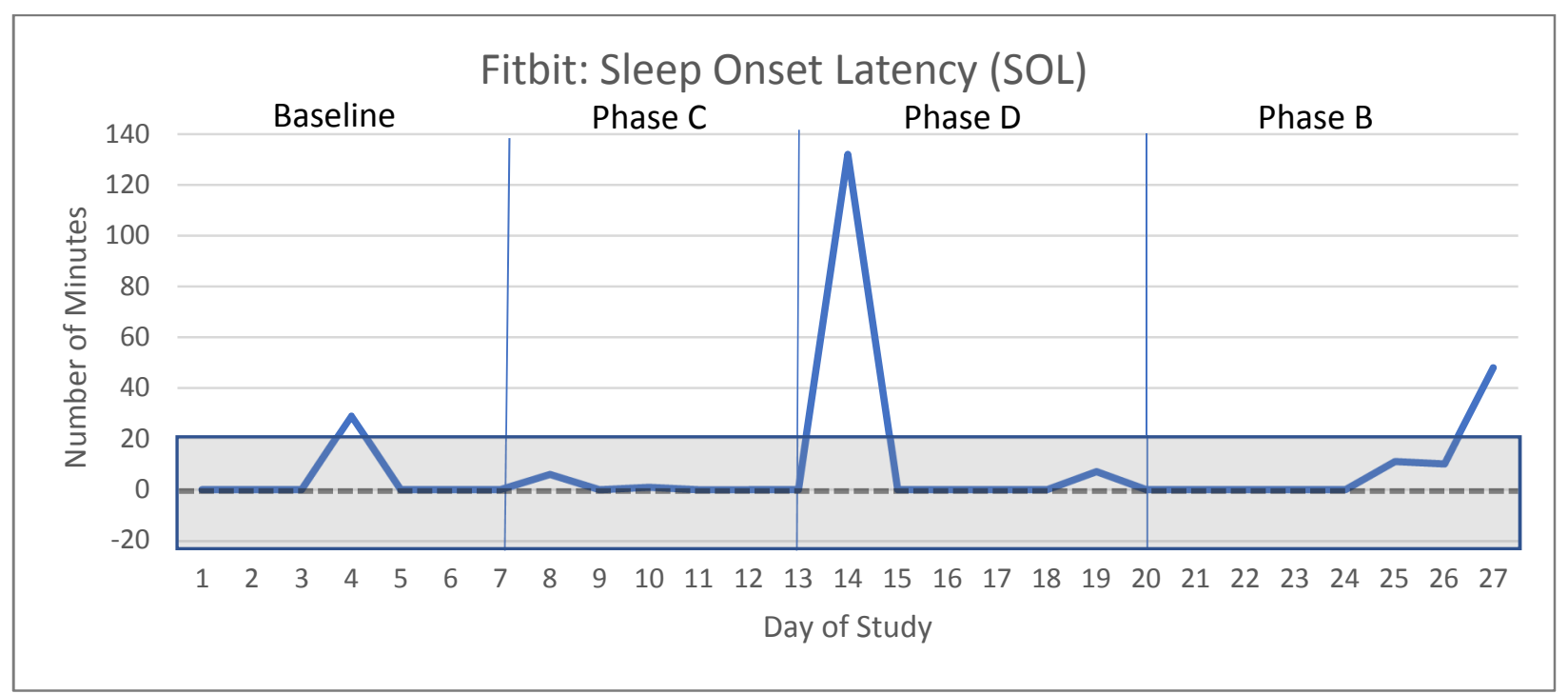

Figure 31. Participant Two Fitbit Sleep Onset Latency. Dashed lines represent the mean of the baseline phase. The gray squared box represents the Two Standard Deviation Band.

Trend. Data for sleep onset latency showed no trend for baseline data or intervention phases $\mathrm{C}$ and $\mathrm{D}$ with an accelerating trend at the end of intervention phase $\mathrm{B}$. The direction of change was linear in phase $\mathrm{B}$, indicating a more consistent rate of change in this phase.

Variability. The direction of change showed mild variability between phases, indicating overall fairly stable data but with minimal change noted.

Inferential statistics. Using the two standard deviation band method, only two nonconsecutive data points fell outside of the two standard deviation band. This indicated no statistically significant change in sleep onset latency.

Comparison of sleep diary and Fitbit data. Data were collected simultaneously throughout the study using both the Consensus Sleep Diary and the Fitbit Inspire HR for each of 
the sleep metrics measured in the study and in each phase of the study. Figure 32 depicts a graphical comparison of these data for sleep onset latency for participant two.

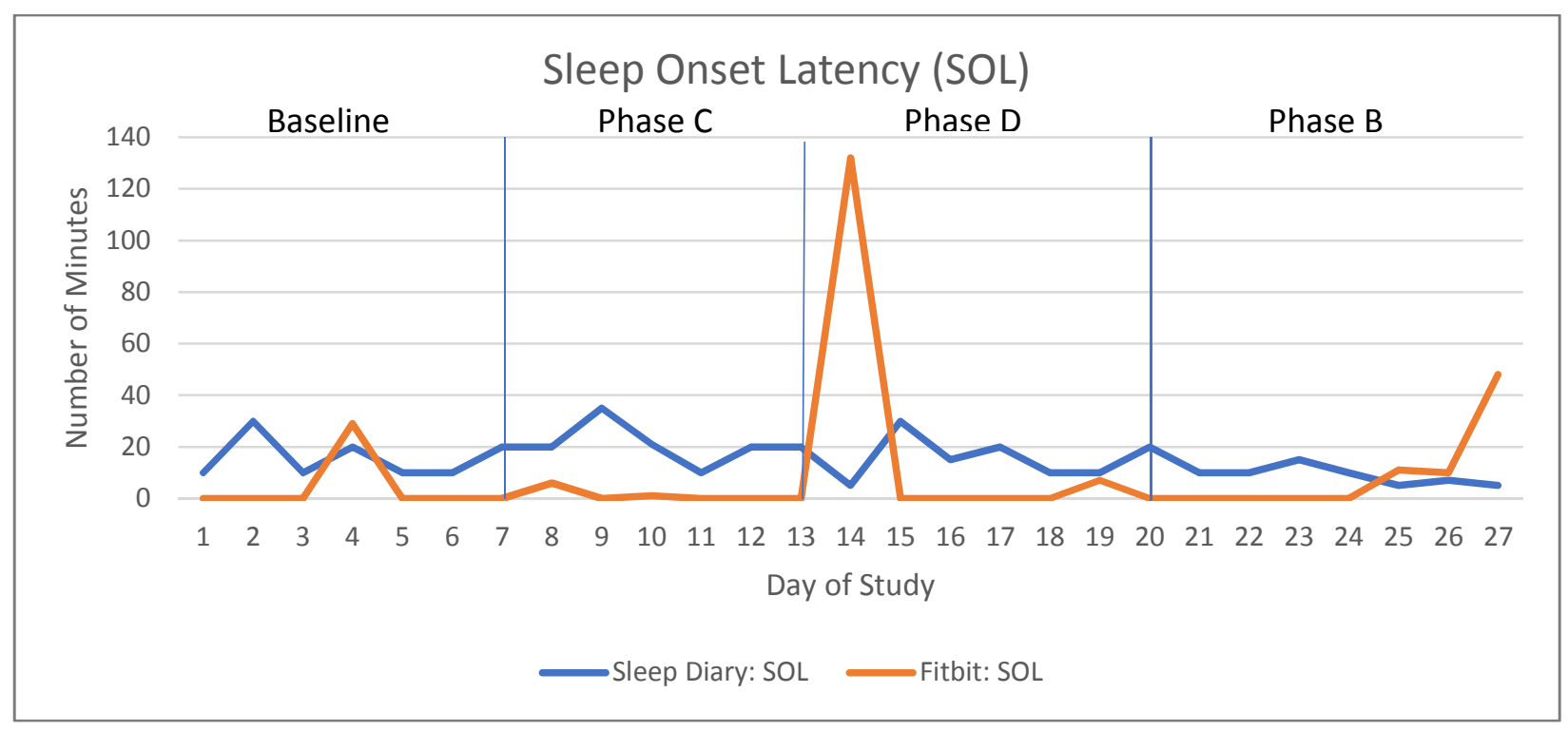

Figure 32. Participant Two Combined Data Sleep Onset Latency.

When comparing data for sleep onset latency from the sleep diary and the Fitbit, there was no consistent trend between the two outcome measures.

\section{Total Sleep Time}

Total sleep time is graphically depicted in Figure 33 for sleep diary data and in Figure 38 for Fitbit data.

Consensus Sleep Diary. Figure 33 depicts total sleep time for all phases of the study using sleep diary data for participant two. Using visual analysis techniques of the graph in Figure 33, the level, trend, slope, and variability were calculated for total sleep time from the sleep diary data.

Level. The mean value for total sleep time of the baseline phase is depicted in Figure 33, which was 412.86 . The mean for phase $\mathrm{C}$ was 438.17 , phase $\mathrm{D}$ was 403.71 , and for phase $\mathrm{B}$ 
497.51. This indicated that the average total sleep time increased for the first and last intervention phases of phases $\mathrm{C}$ and $\mathrm{B}$ and decreased slightly for intervention phase $\mathrm{D}$.

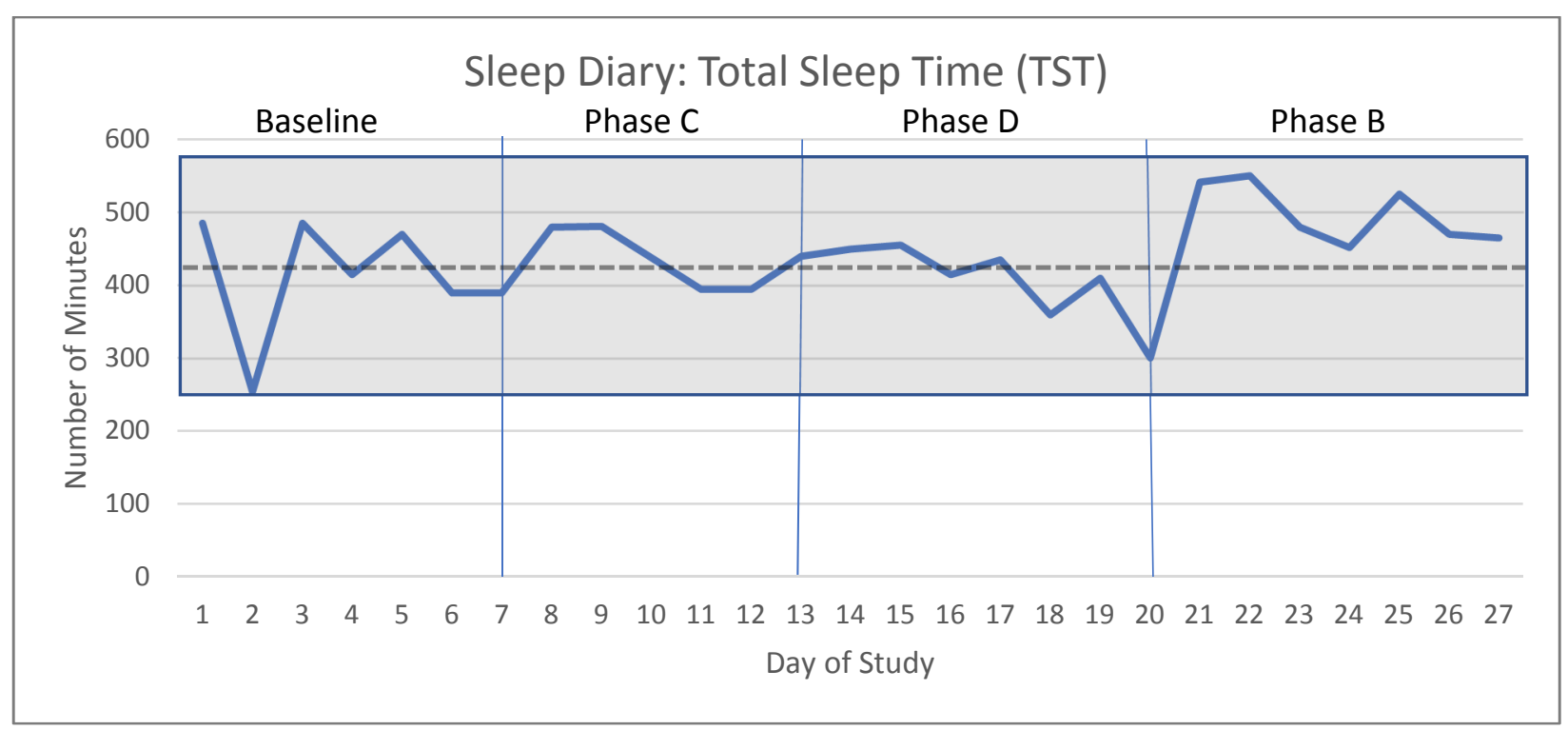

Figure 33. Participant Two Sleep Diary Sleep Total Sleep Time. Dashed lines represent the mean of the baseline phase. The gray squared box represents the Two Standard Deviation Band.

Trend. Data for total sleep time showed no trend for baseline data and intervention phase $\mathrm{B}$ and a decelerating trend within intervention phases $\mathrm{C}$ and $\mathrm{D}$, indicating overall fluctuations in total sleep time throughout the study. However, the overall trend between the baseline phase and the last intervention phase of the study showed an accelerating trend, or an increase in total sleep time at the end of the study as compared to baseline data. The direction of change was curvilinear in phase $\mathrm{C}$ and linear in phase $\mathrm{D}$, indicating a more consistent rate of change in these phases. A celeration line was calculated for total sleep time using the sleep diary data and is graphically depicted in Figure 34. Each of the data points in all of the intervention phases fell above the celeration line, indicating a significant change in Daniel's response pattern during the intervention phases when compared to the baseline phase. 


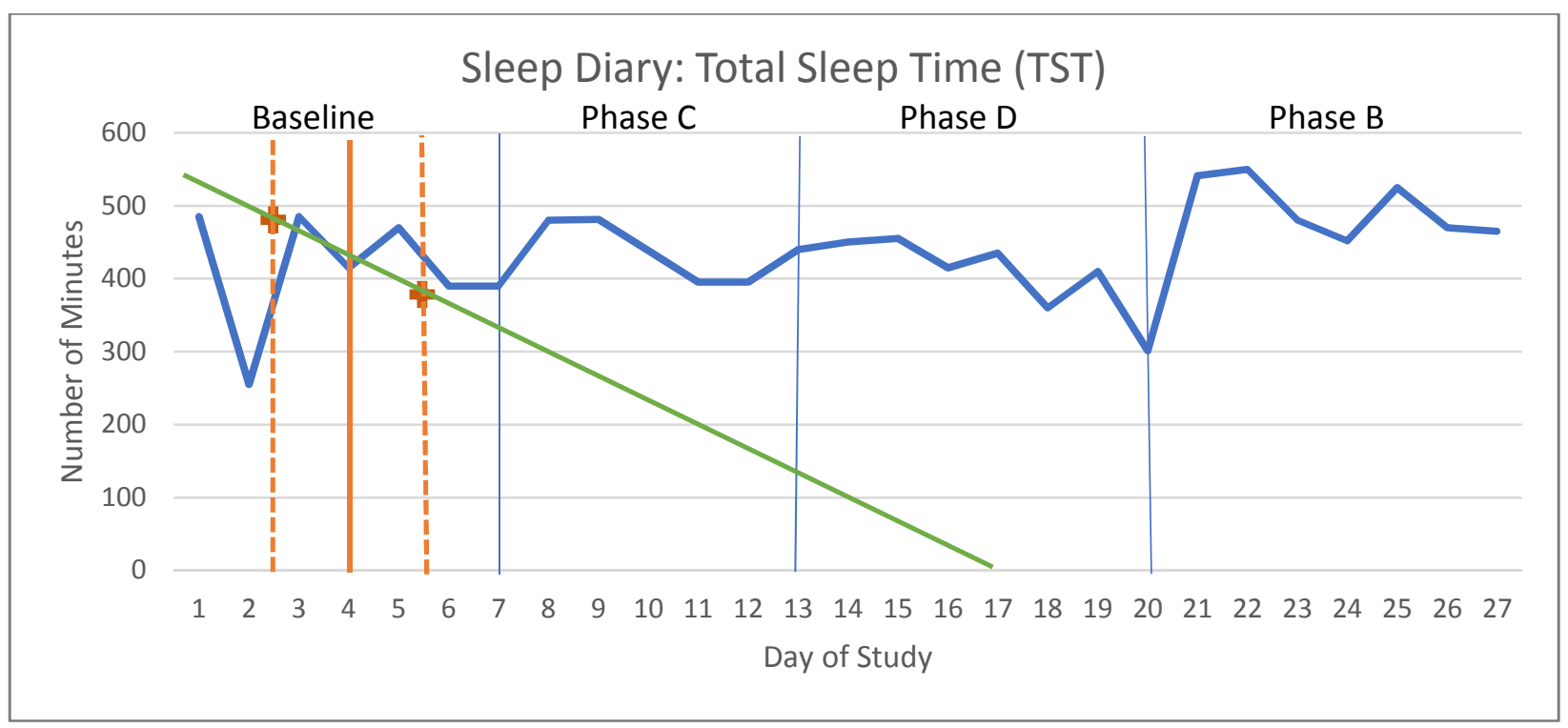

Figure 34. Participant Two Total Sleep Time Celeration Line. The solid orange line represents a dividing line for half of the baseline data. Dashed orange lines represent a dividing line for quarters of the baseline data. The solid green line represents the celeration line.

Slope. The slope of the trend line was calculated for each of the phases to show the change in the angle of the trend. A comparison between the intervention phases was done by calculating the slope for each phase individually. The slope of data in phase $\mathrm{C}$ was 1.4 and decelerating, indicating that total sleep time decreased at an average rate of 0.92 times per week during this phase. The slope of data in phase $\mathrm{D}$ was 2.0 and decelerating, indicating that total sleep time decreased at an average rate of 2.0 times per week during this phase. The slope of data in phase B was 1.37 and decelerating, indicating that total sleep time decreased at an average rate of 1.37 times per week during this phase. Decelerating slopes for each intervention phase indicated decreases in total sleep time in each intervention phase with the most significant rate of change noted in intervention phase $\mathrm{D}$. The slope of total sleep time data for each intervention phase is visually depicted in Figures 35, 36, and 37. 


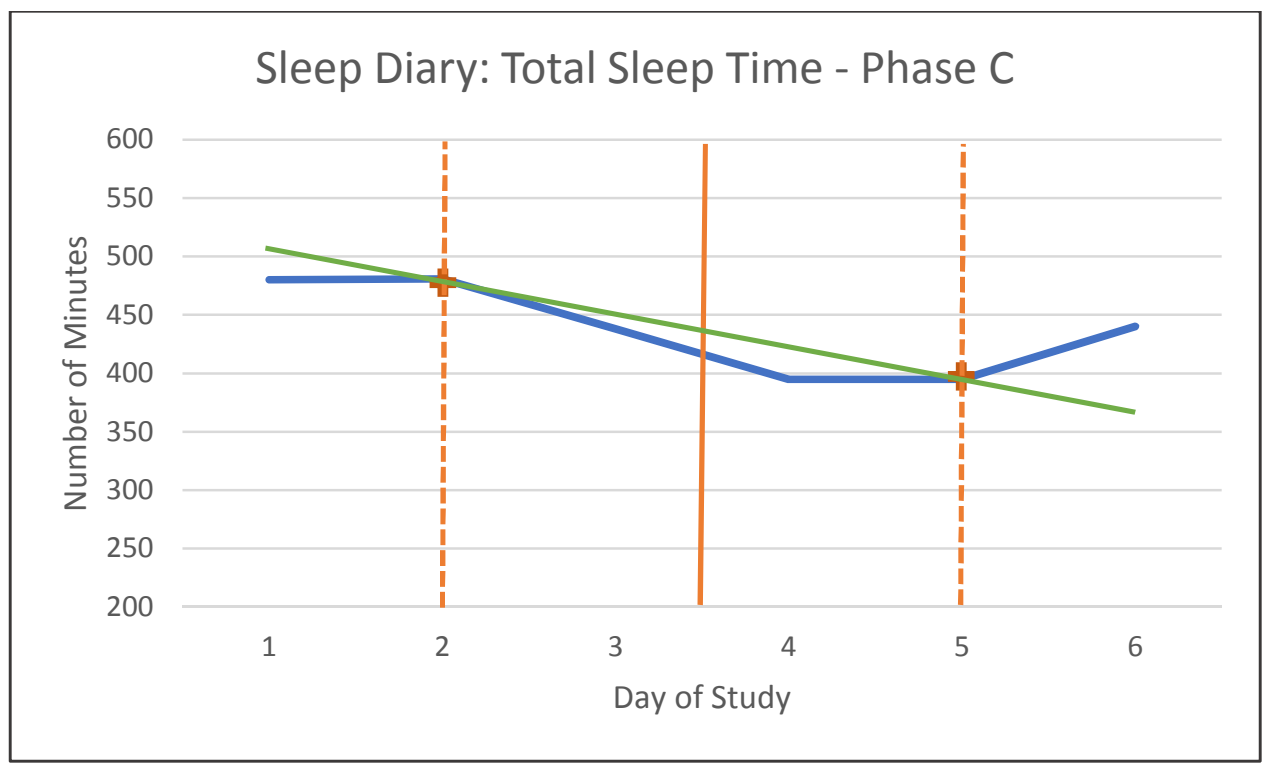

Figure 35. Participant Two Sleep Diary Total Sleep Time Slope Phase C. The solid orange line represents a dividing line for half of the baseline data. Dashed orange lines represent a dividing line for quarters of the baseline data. The solid green line represents the celeration line.

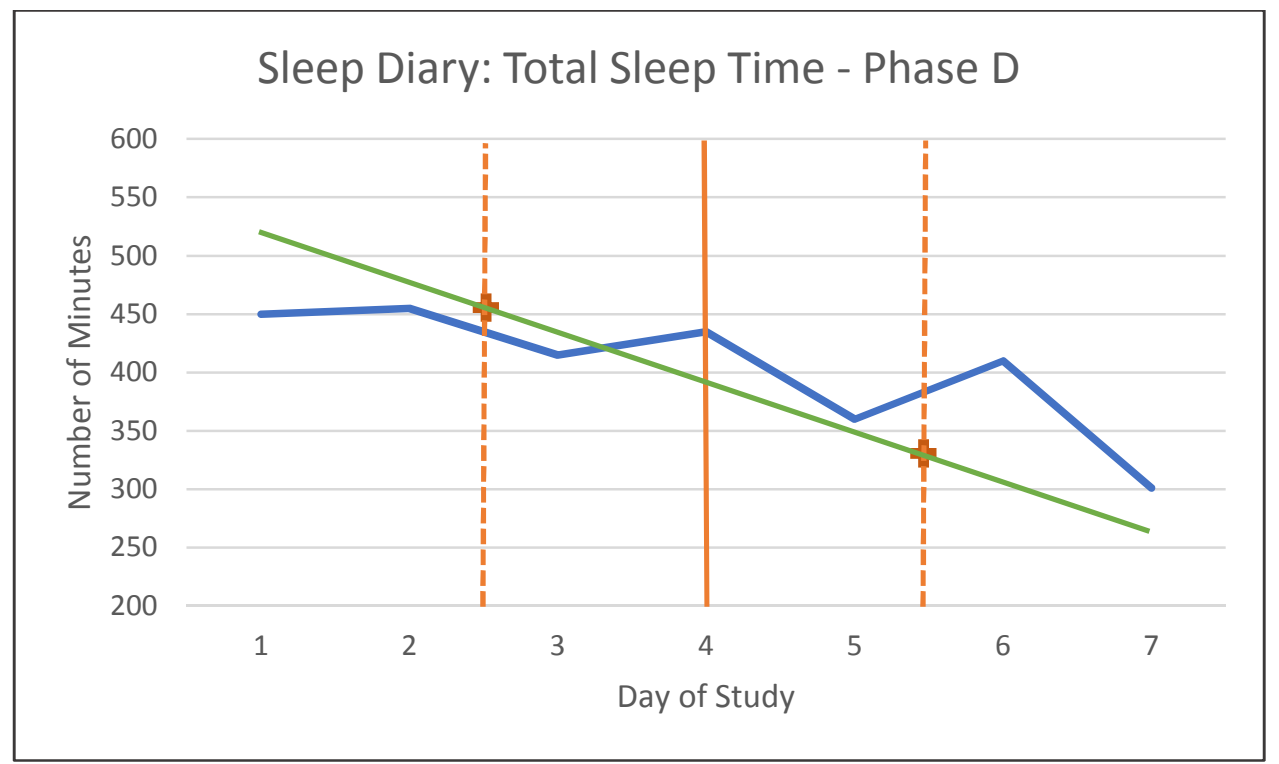

Figure 36. Participant Two Sleep Diary Total Sleep Time Slope Phase D. The solid orange line represents a dividing line for half of the baseline data. Dashed orange lines represent a dividing line for quarters of the baseline data. The solid green line represents the celeration line. 


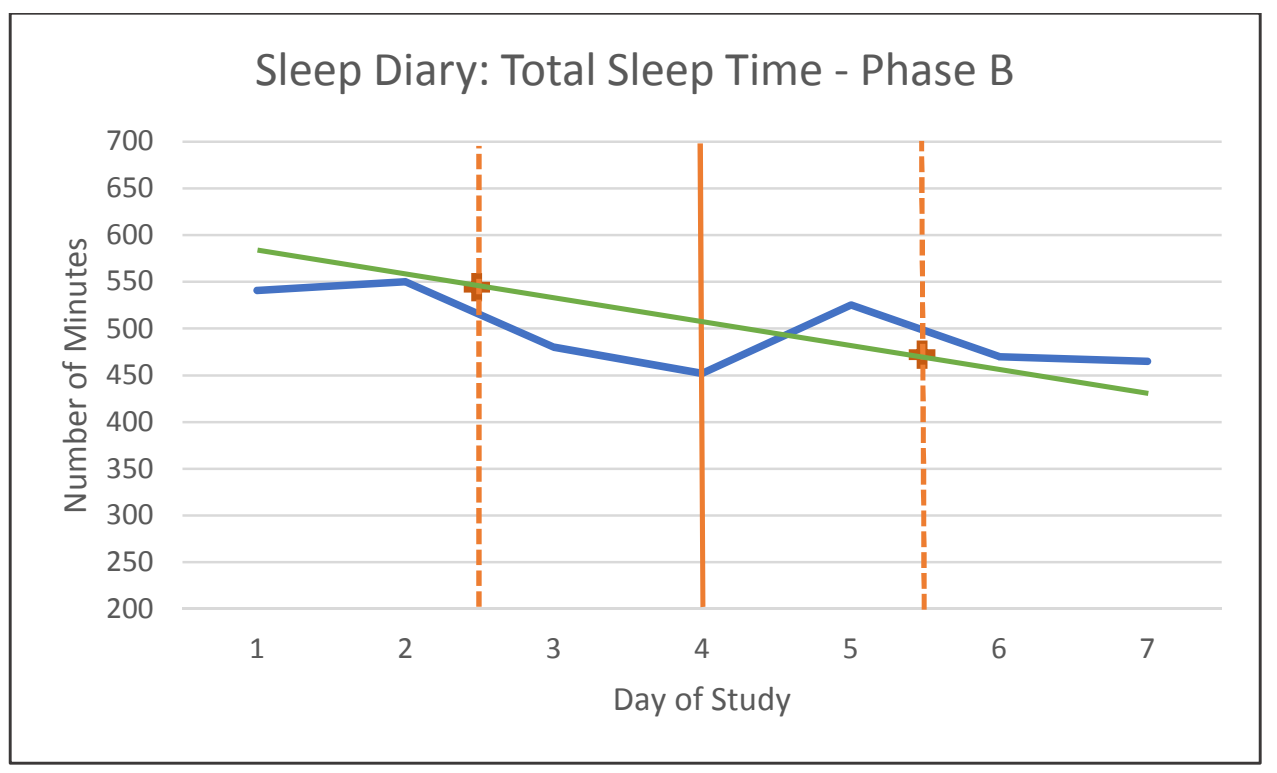

Figure 37. Participant Two Sleep Diary Total Sleep Time Slope Phase B. The solid orange line represents a dividing line for half of the baseline data. Dashed orange lines represent a dividing line for quarters of the baseline data. The solid green line represents the celeration line.

Variability. The direction of change was variable throughout the phases with less variability noted in phases $\mathrm{C}$ and $\mathrm{D}$, indicating more stable data in those phases.

Inferential statistics. Using the two standard deviation band method, no data points fell outside of the two standard deviation band. This indicated no statistically significant change in total sleep time. The $\mathrm{C}$ statistic was calculated for the baseline phase, which yielded $\mathrm{C}=-0.548$ with a standard error of 0.3227 . The $z$ score was $1.69(1.69>1.64)$, indicating a significant trend in the baseline data and making it more difficult to assess a significant trend across baseline and intervention phases. Therefore, additional c-statistic calculations were not completed for this variable.

Fitbit Inspire HR. Figure 38 depicts total sleep time for all phases of the study using Fitbit data for participant two. 


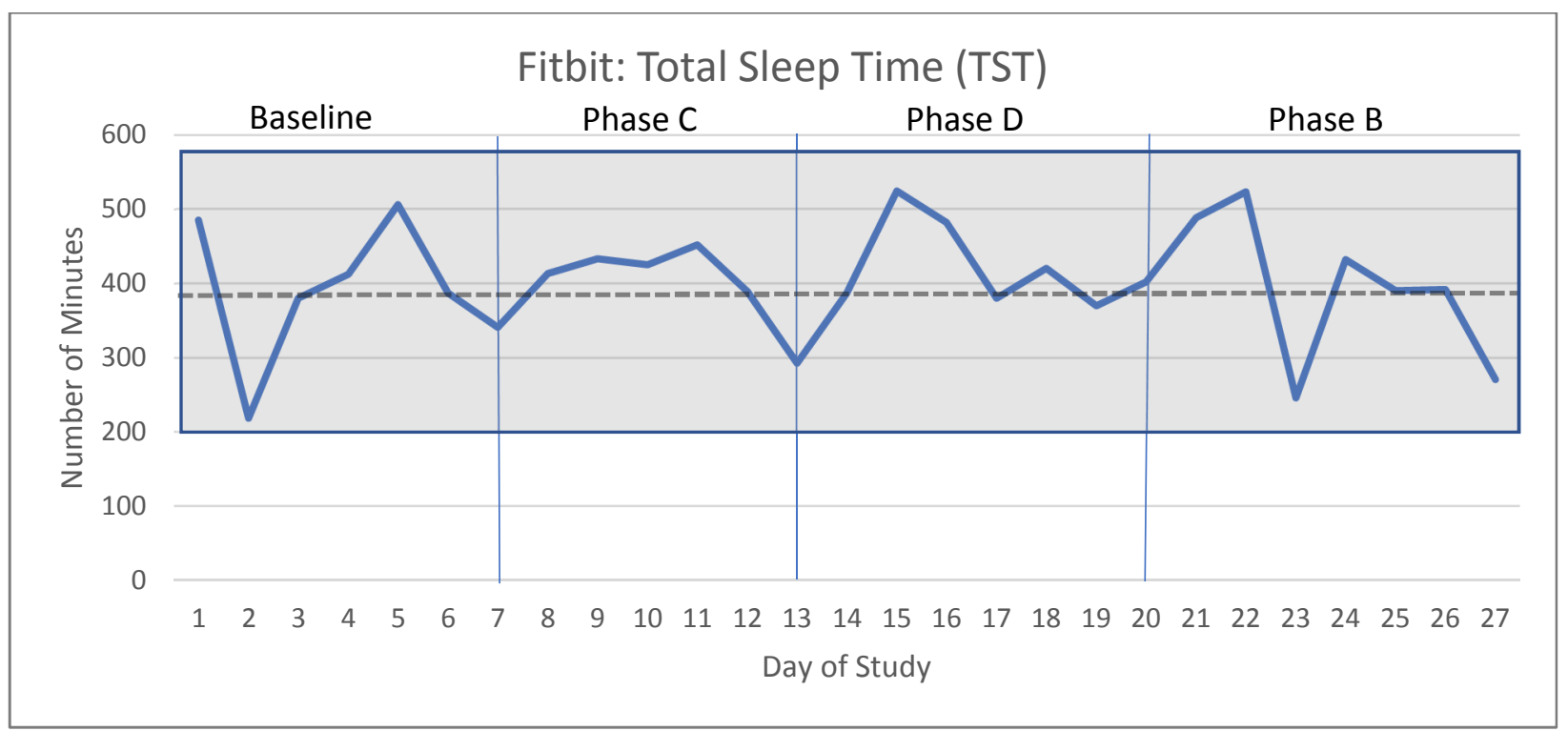

Figure 38. Participant Two Fitbit Total Sleep Time. Dashed lines represent the mean of the baseline phase. The gray squared box represents the Two Standard Deviation Band.

Using visual analysis techniques of the graph in Figure 38, the level, trend, slope, and variability were calculated for total sleep time from the Fitbit data.

Level. The mean value for total sleep time of the baseline phase is depicted in Figure 38, which was 390.0. The mean for phase $\mathrm{C}$ was 400.83 , phase $\mathrm{D}$ was 423.57 , and for phase $\mathrm{B}$ 391.71. This indicates that the average total sleep time increased for all intervention phases when compared to the baseline, with the most significant increase in phase D.

Trend. Data for sleep efficiency showed no trend for baseline data and intervention phase $\mathrm{D}$ and a mild decelerating trend within intervention phases $\mathrm{C}$ and $\mathrm{B}$, indicating fluctuations in total sleep time throughout the study. The direction of change was curvilinear in phase $\mathrm{C}$, indicating a more consistent rate of change in this phase.

Variability. The direction of change was variable throughout with less variability noted in phase $\mathrm{C}$, which indicated more stable data in this phase. 
Inferential statistics. Using the two standard deviation band method, no data points fell outside of the two standard deviation band. This indicated no statistically significant change in total sleep time.

Comparison of sleep diary and Fitbit data. Data were collected simultaneously throughout the study using both the Consensus Sleep Diary and the Fitbit Inspire HR for each of the sleep metrics measured in the study and in each phase of the study. Figure 39 depicts a graphical comparison of these data for total sleep time for participant two.

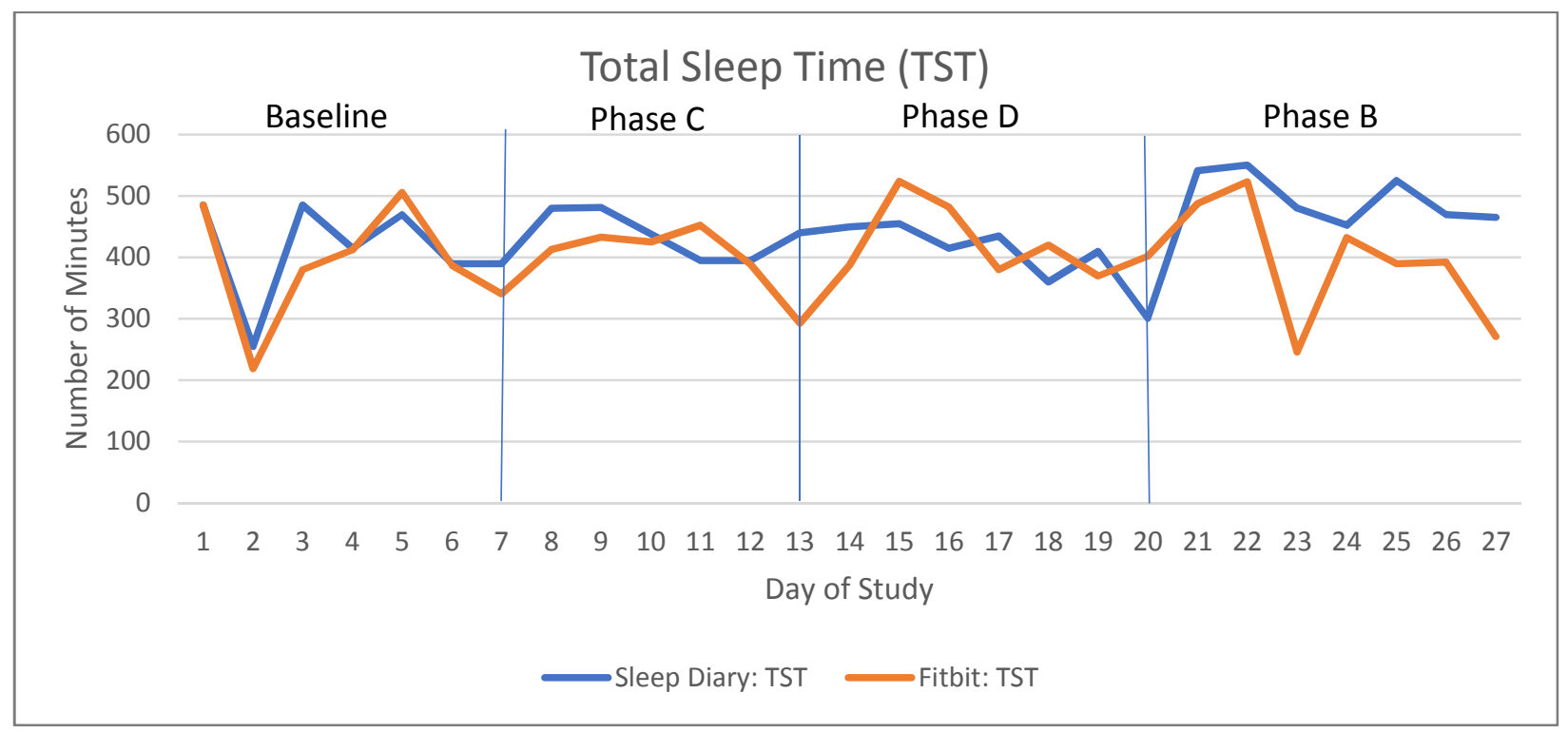

Figure 39. Participant Two Combined Data Total Sleep Time.

When comparing data for total sleep time from the sleep diary and the Fitbit, the trend of data was overall relatively similar from phase to phase with the exception that the mean total sleep time decreased in phase $\mathrm{D}$ with sleep diary data. All other phases showed an increase in total sleep time for both outcome measures when comparing the mean of the intervention phases to the mean of the baseline phase. 


\section{Sleep Efficiency}

Sleep efficiency is graphically depicted in Figure 40 for sleep diary data and in Figure 45 for Fitbit data.

Consensus Sleep Diary. Figure 40 depicts sleep efficiency for all phases of the study using sleep diary data for participant two.

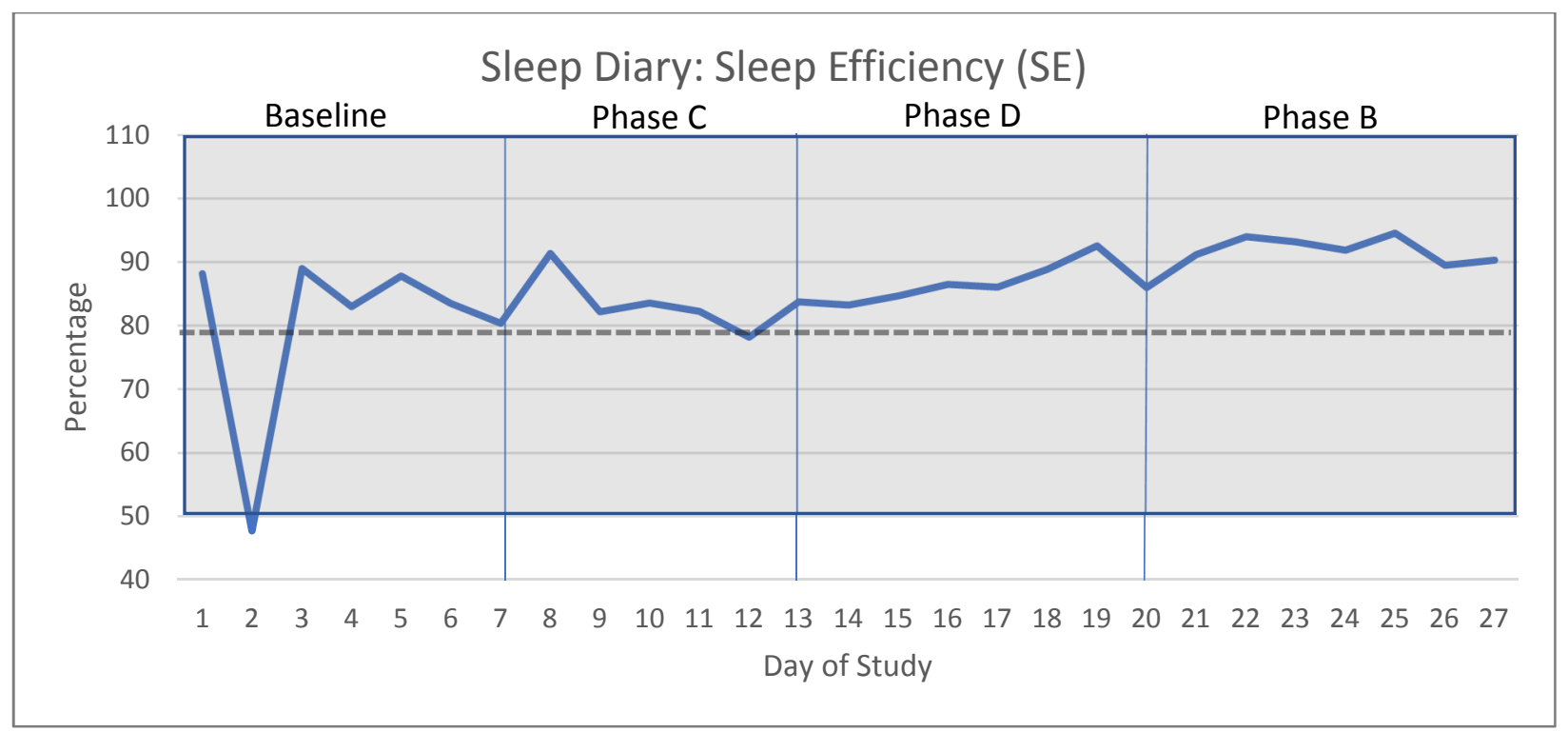

Figure 40. Participant Two Sleep Diary Sleep Efficiency. Dashed lines represent the mean of the baseline phase. The gray squared box represents the Two Standard Deviation Band.

Using visual analysis techniques of the graph in Figure 40, the level, trend, slope, and variability were calculated for sleep efficiency from the sleep diary data.

Level. The mean value for sleep efficiency of the baseline phase is depicted in Figure 40, which was 79.943 . The mean for phase $\mathrm{C}$ was 83.583 , phase $\mathrm{D}$ was 86.871 , and for phase $\mathrm{B}$ 92.100. This indicates that the average of sleep efficiency progressively increased throughout the intervention phases.

Trend. Data for sleep efficiency showed no trend for baseline data and intervention phase $\mathrm{C}$ and a mild accelerating trend for intervention phases $\mathrm{D}$ and $\mathrm{B}$, indicating a mild 
improvement in total sleep time as the study progressed. The direction of change was variable throughout the phases and was linear in phase D. A celeration line was calculated for sleep efficiency using the sleep diary data and is graphically depicted in Figure 41.

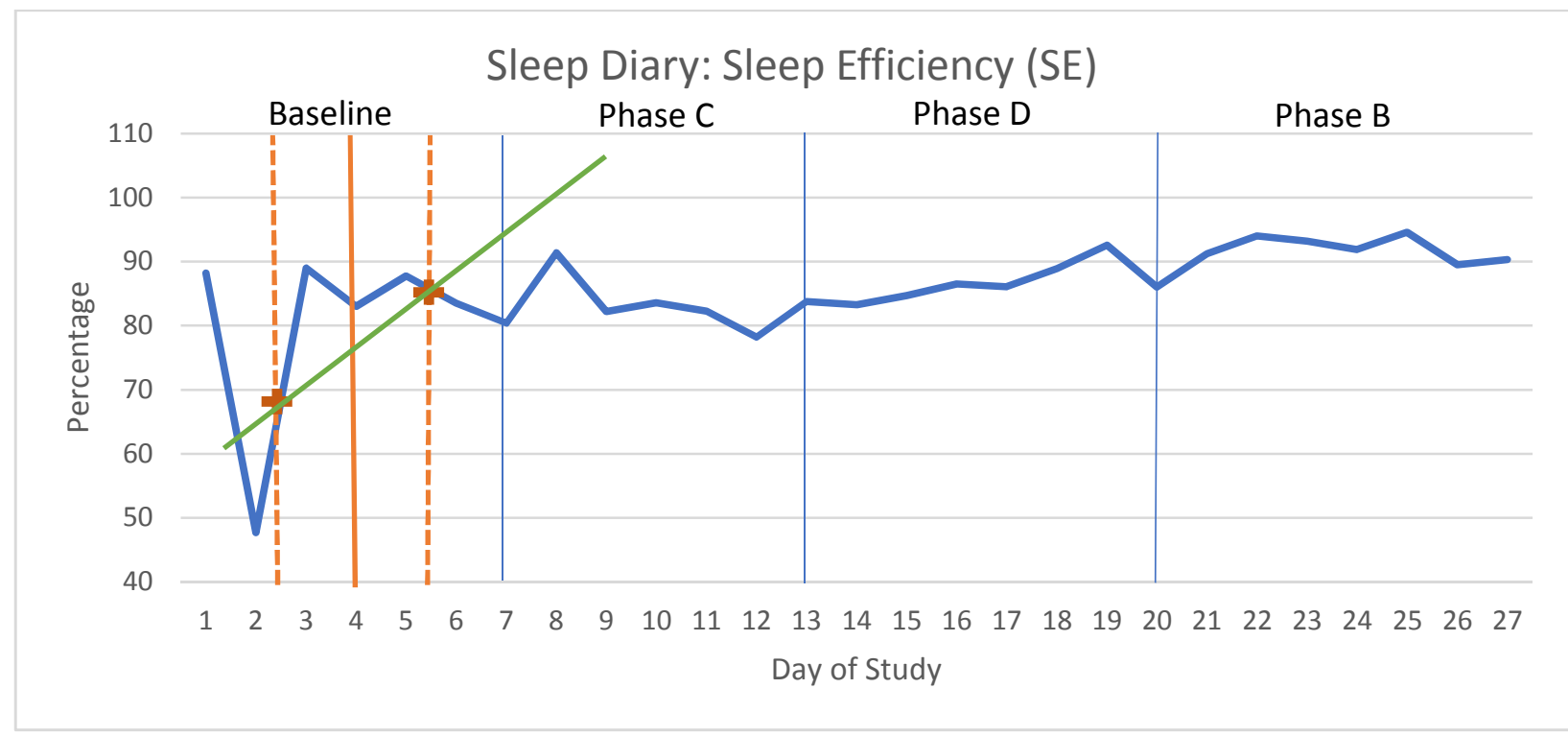

Figure 41. Participant Two Sleep Diary Sleep Efficiency Trend. The solid orange line represents a dividing line for half of the baseline data. Dashed orange lines represent a dividing line for quarters of the baseline data. The solid green line represents the celeration line.

Each of the data points in all of the intervention phases fell below the celeration line, indicating a significant change in Daniel's response pattern during the intervention phases when compared to the baseline phase.

Slope. The slope of data in phase C was 1.02 and decelerating, indicating that sleep efficiency decreased at an average rate of 1.02 times per week during this phase. The slope of data in phase D was 1.05 and accelerating, indicating that sleep efficiency increased at an average rate of 1.05 times per week during this phase. The slope of data in phase B was 1.11 and decelerating, indicating that sleep efficiency decreased at an average rate of 1.11 times per week during this phase. This indicated positive changes in sleep efficiency only in intervention phase 
D. The slope of sleep efficiency data for each intervention phase is visually depicted in Figures 42, 43, and 44.

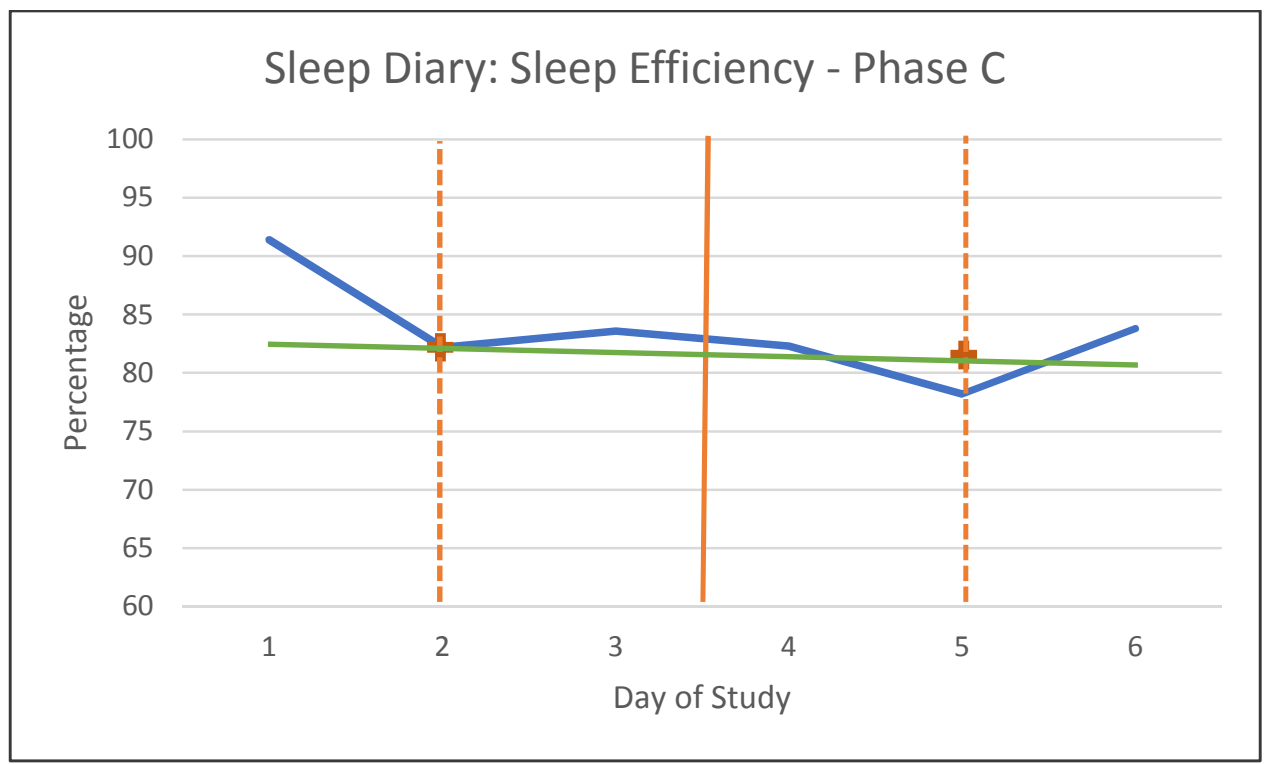

Figure 42. Participant Two Sleep Diary Sleep Efficiency Slope Phase C. The solid orange line represents a dividing line for half of the baseline data. Dashed orange lines represent a dividing line for quarters of the baseline data. The solid green line represents the celeration line.

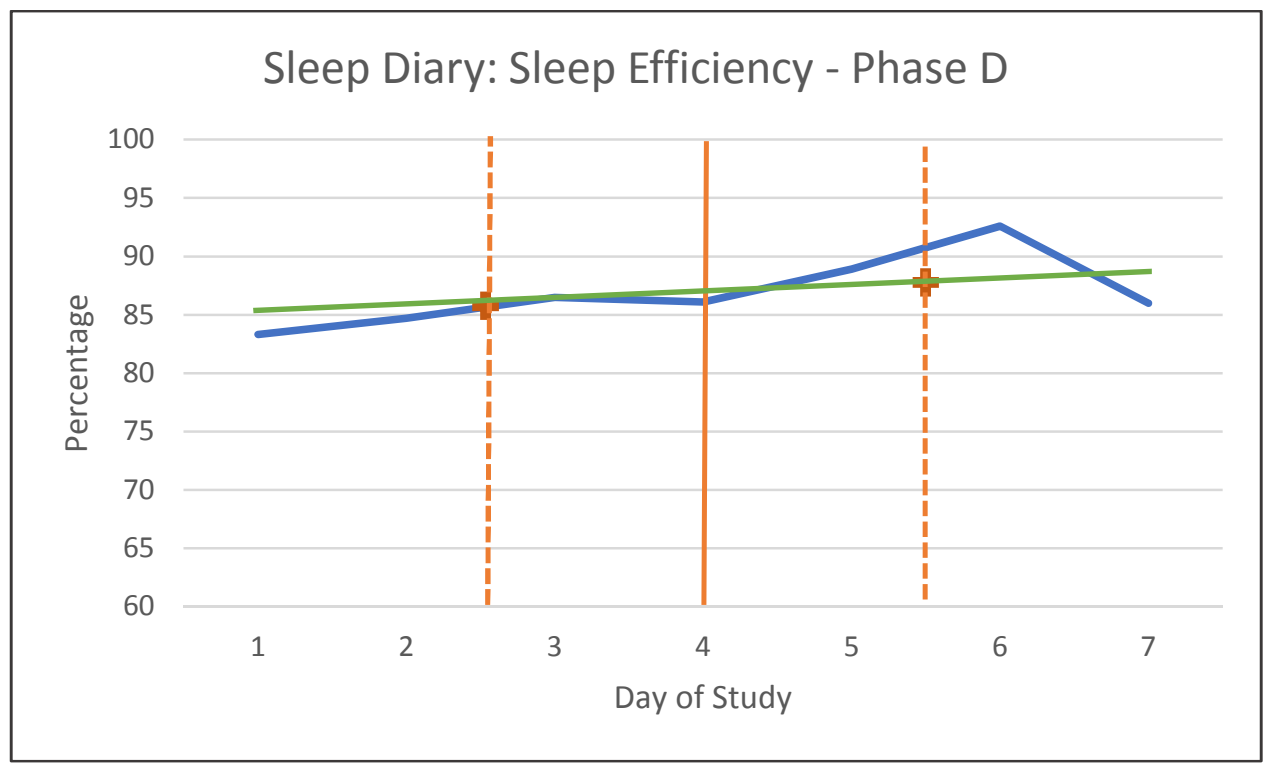

Figure 43. Participant Two Sleep Diary Sleep Efficiency Slope Phase D. The solid orange line represents a dividing line for half of the baseline data. Dashed orange lines represent a dividing line for quarters of the baseline data. The solid green line represents the celeration line. 


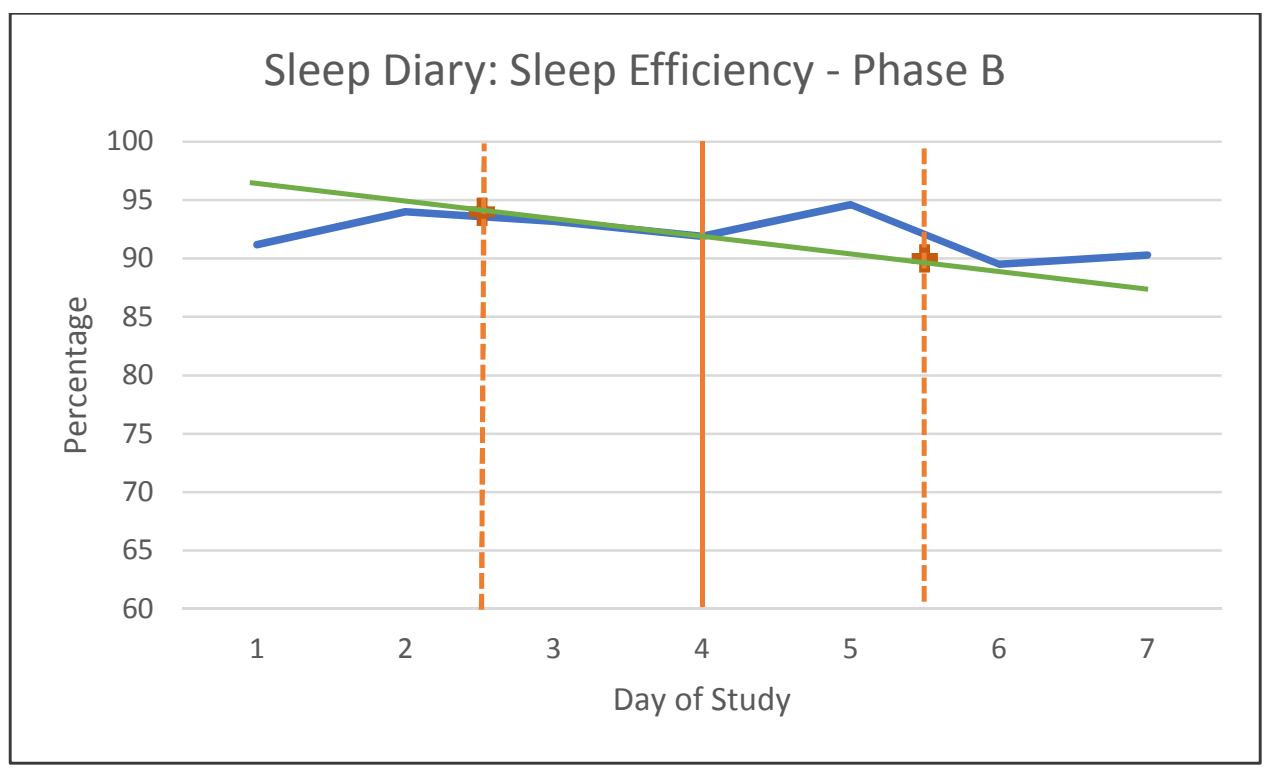

Figure 44. Participant Two Sleep Diary Sleep Efficiency Slope Phase B. The solid orange line represents a dividing line for half of the baseline data. Dashed orange lines represent a dividing line for quarters of the baseline data. The solid green line represents the celeration line.

Variability. The direction of change showed mild variability throughout the intervention phases, indicating relatively stable data in the intervention phases.

Inferential statistics. Using the two standard deviation band method, no data points in the intervention phases fell outside of the two standard deviation band. This indicated no statistically significant change in sleep efficiency. The C statistic was calculated for the baseline phase, which yielded $\mathrm{C}=-0.3476$ with a standard error of 0.3227 . The $\mathrm{z}$ score was $1.077(1.077$ $<1.64$ ), indicating no significant trend in the baseline data. Baseline data were then combined with data from the intervention phases, yielding $\mathrm{C}=0.042$ with a standard error of 0.185 . The $\mathrm{z}$ score for the combined data was $0.227(0.227<1.64)$, indicating no significant trend across baseline and intervention phases.

Fitbit Inspire HR. Figure 45 depicts sleep efficiency for all phases of the study using Fitbit data for participant two. 


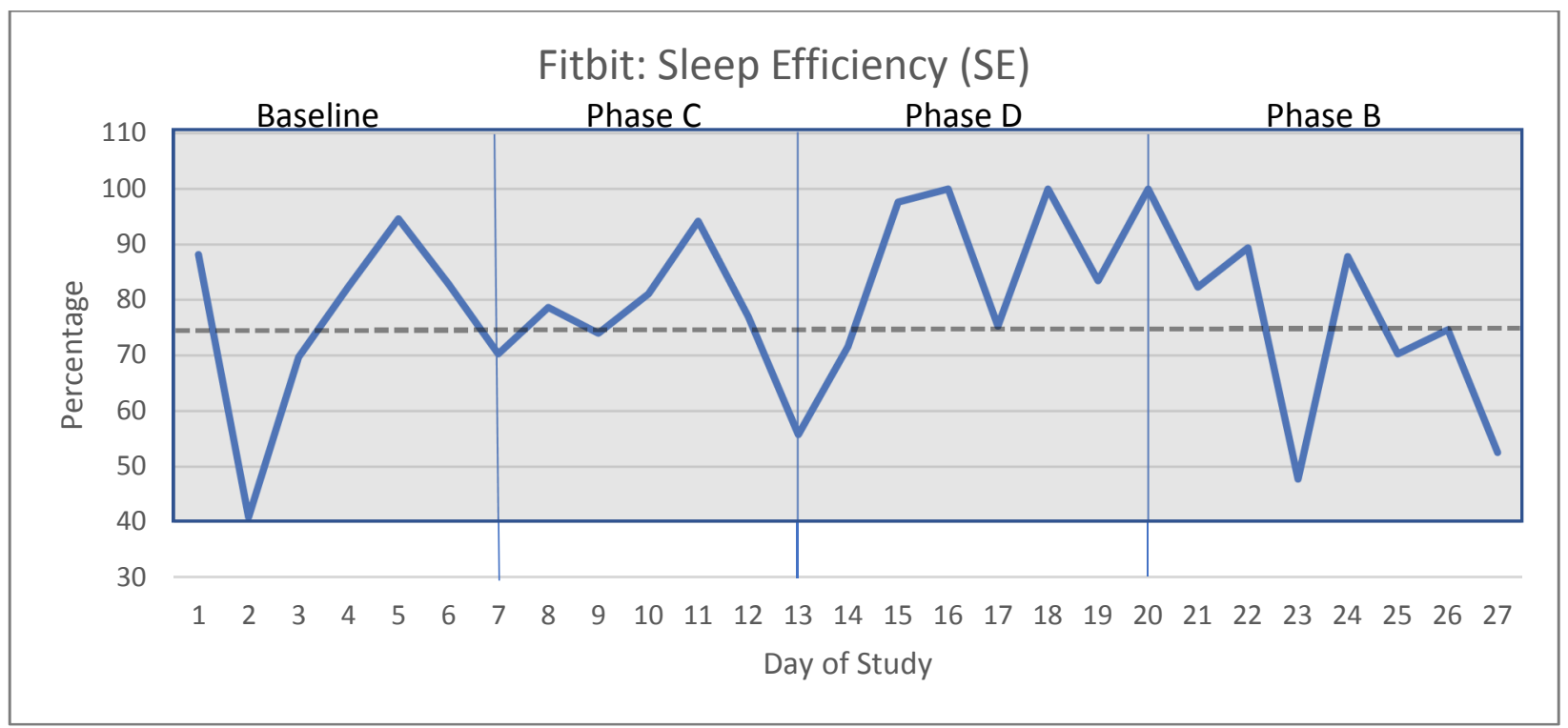

Figure 45. Participant Two Fitbit Sleep Efficiency. Dashed lines represent the mean of the baseline phase. The gray squared box represents the Two Standard Deviation Band.

Using visual analysis techniques of the graph in Figure 45, the level, trend, slope, and variability were calculated for sleep efficiency from the Fitbit data.

Level. The mean value for sleep efficiency of the baseline phase is depicted in Figure 45, which was 75.571 . The mean for phase $\mathrm{C}$ was 76.80 , phase $\mathrm{D}$ was 89.729 , and for phase $\mathrm{B}$ 72.129. This indicates that the average of sleep efficiency increased for the first two intervention phases of phases $\mathrm{C}$ and $\mathrm{D}$ and decreased slightly for the last intervention phase during phase $\mathrm{B}$.

Trend. Data for sleep efficiency showed no trend for baseline data and interventions phases $\mathrm{C}$ and $\mathrm{D}$ and a decelerating trend in intervention phase $\mathrm{B}$. However, the overall trend of the data showed a gradual increase in sleep efficiency from baseline through interventions $\mathrm{C}$ and $\mathrm{D}$ and then a decline in sleep efficiency in phase B until the end of the study.

Variability. The direction of change was variable throughout the phases with less variability noted in phase $\mathrm{D}$, indicating more stable data in that phase. 
Inferential statistics. Using the two standard deviation band method, no data points fell outside of the two standard deviation band. This indicated no statistically significant change in sleep efficiency.

Comparison of sleep diary and Fitbit data. Data were collected simultaneously throughout the study using both the Consensus Sleep Diary and the Fitbit Inspire HR for each of the sleep metrics measured in the study and in each phase of the study. Figure 46 depicts a graphical comparison of these data for sleep efficiency for participant two.

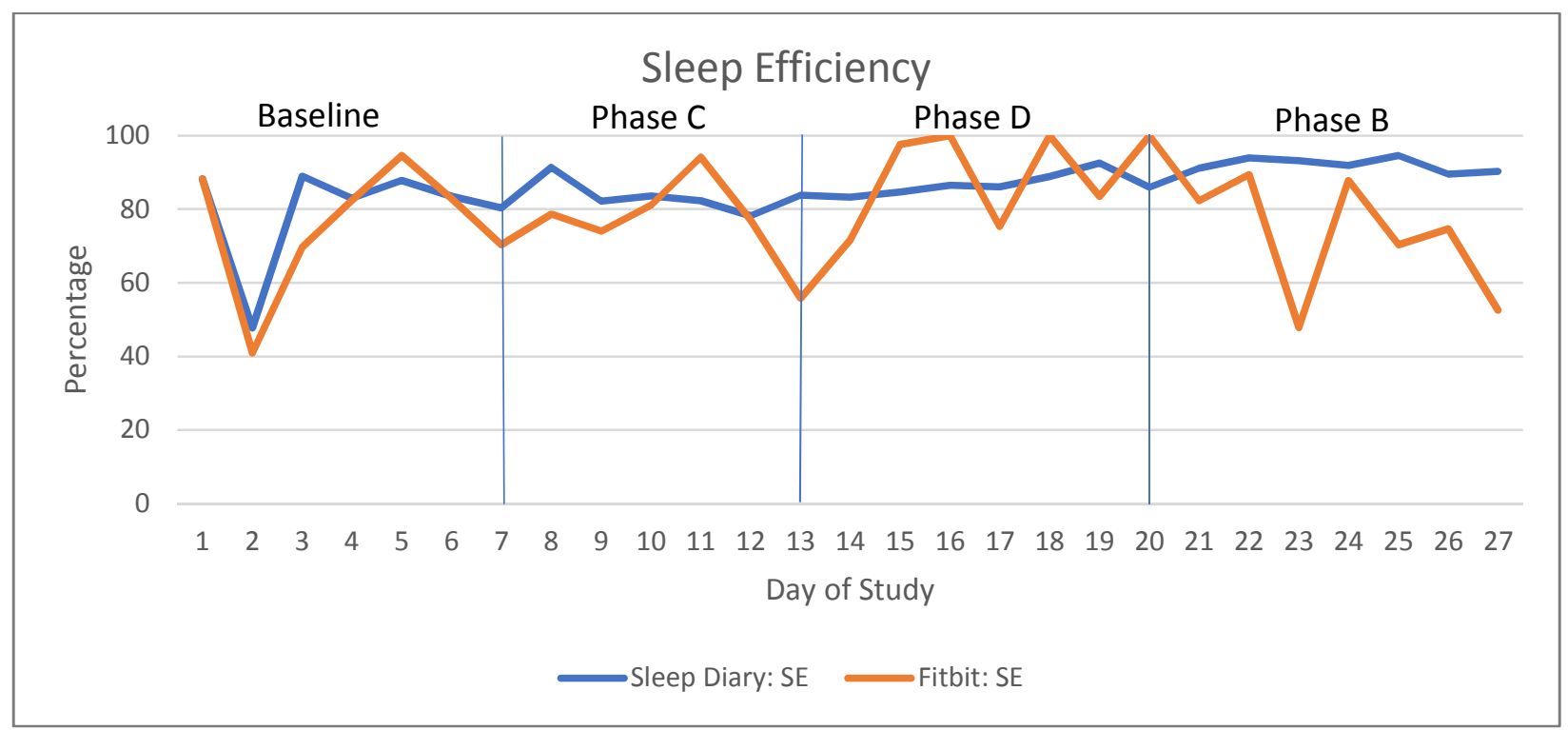

Figure 46. Participant Two Combined Data Sleep Efficiency.

When comparing data for sleep efficiency from the sleep diary and the Fitbit, there was a gradual increase in sleep efficiency across all phases of the study with sleep diary data and in the first two intervention phases, phases $\mathrm{C}$ and $\mathrm{D}$, with Fitbit data. However, there was a decrease in sleep efficiency for phase B based on Fitbit data.

\section{Number of Awakenings}

The number of awakenings is graphically depicted in Figure 47 for sleep diary data and in Figure 48 for Fitbit data. 
Consensus Sleep Diary. Figure 47 depicts the number of awakenings for all phases of the study using sleep diary data for participant two.

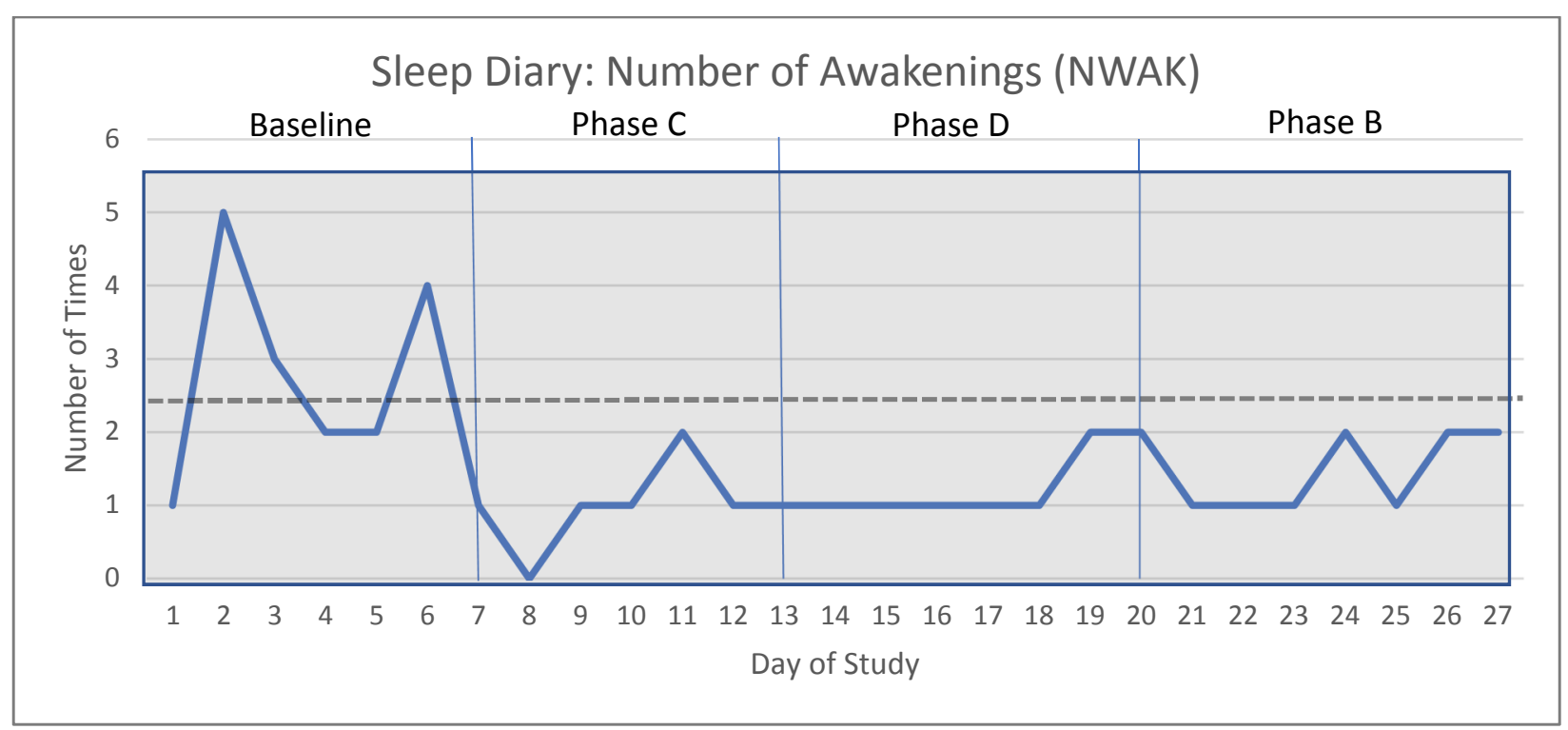

Figure 47. Participant Two Sleep Diary Number of Awakenings. Dashed lines represent the mean of the baseline phase. The gray squared box represents the Two Standard Deviation Band.

Using visual analysis techniques of the graph in Figure 47, the level, trend, slope, and variability were calculated for the number of awakenings from the sleep diary data.

Level. The mean value for the number of awakenings of the baseline phase is depicted in Figure 47 , which was 2.57 . The mean for phase C was 1.0 , phase D was 1.29 , and for phase B 1.43. This indicates that the average number of awakenings decreased for each of the intervention phases when compared to the baseline.

Trend. Data for the number of awakenings showed no trend for baseline data and intervention phases $\mathrm{C}$ and $\mathrm{B}$ and a mild accelerating trend within intervention phase $\mathrm{D}$, indicating fluctuations in the number of awakenings throughout the study. However, the overall trend from baseline to intervention phases was decelerating, showing an overall decrease in the 
number of awakenings from baseline to the end of the study. The direction of change was variable throughout the phases and was linear at the end of phase D.

Variability. The direction of change was variable throughout the baseline and intervention phases $\mathrm{C}$ and $\mathrm{B}$ with more stable data noted in phase $\mathrm{D}$.

Inferential statistics. Using the two standard deviation band method, no data points fell outside of the two standard deviation band. This indicated no statistically significant change in the number of awakenings.

Fitbit Inspire HR. Figure 48 depicts the number of awakenings for all phases of the study using Fitbit data for participant two.

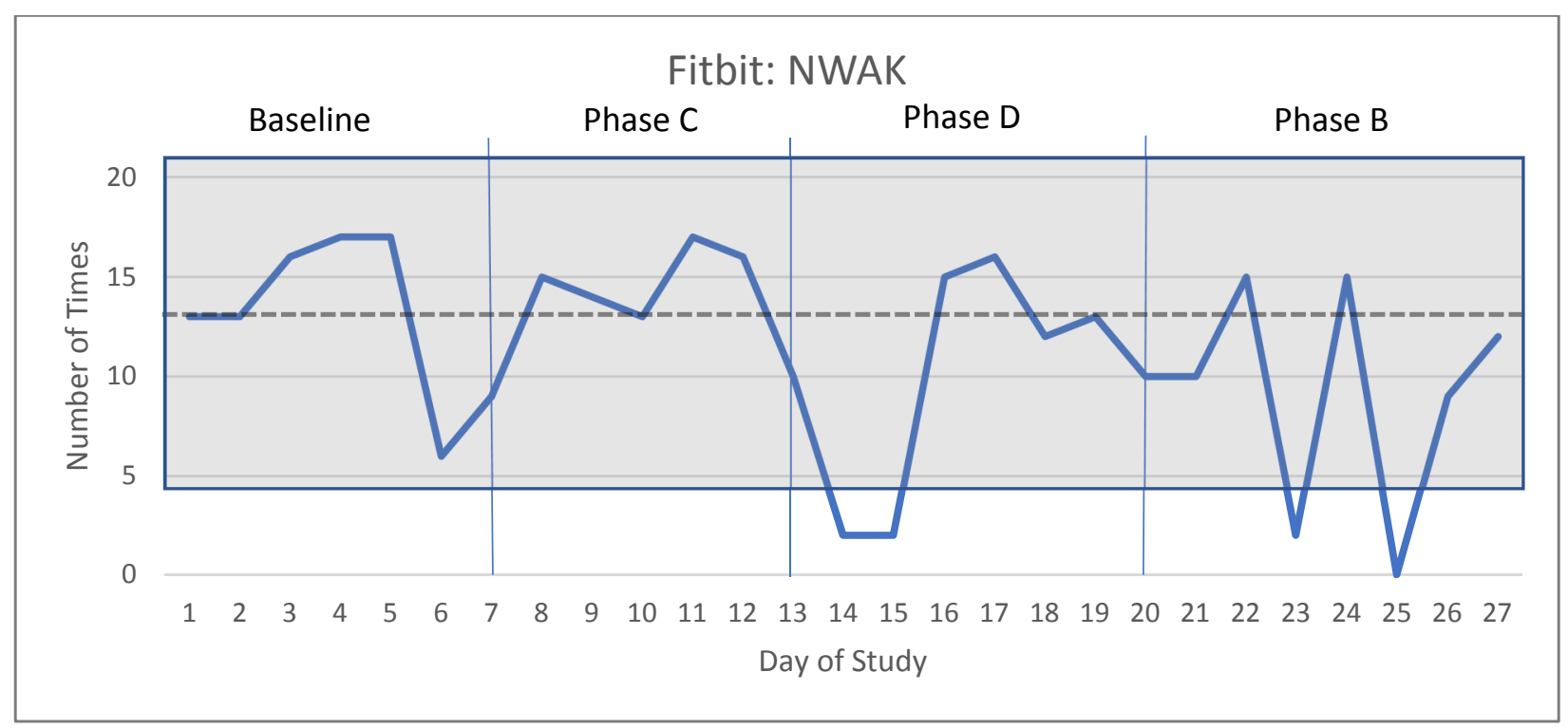

Figure 48. Participant Two Fitbit Number of Awakenings. Dashed lines represent the mean of the baseline phase. The gray squared box represents the Two Standard Deviation Band.

Using visual analysis techniques of the graph in Figure 48, the level, trend, slope, and variability were calculated for the number of awakenings from the Fitbit data.

Level. The mean value for the number of awakenings of the baseline phase is depicted in Figure 48, which was 13.0. The mean for phase $\mathrm{C}$ was 14.17 , phase $\mathrm{D}$ was 10.0 , and for phase $\mathrm{B}$ 
9.0. This indicated that the average number of awakenings slightly increased for the first intervention phase with phase $\mathrm{C}$ but decreased during the last two intervention phases for phases $\mathrm{D}$ and $\mathrm{B}$.

Trend. Data for the number of awakenings showed no trend for baseline data and intervention phases, indicating fluctuations in the number of awakenings throughout the study. There was a mild overall decelerating trend from baseline to intervention phases, showing an overall mild decrease in the number of awakenings from baseline to intervention phases. The direction of change was variable throughout the phases.

Variability. The direction of change was variable throughout the phases with the most variability noted in phase $\mathrm{B}$, which indicated less stable data in that phase.

Inferential statistics. Using the two standard deviation band method, data points from days 14 and 15 fell outside of the two standard deviation band, indicating a statistically significant decrease in the number of awakenings in phase D. Two data points, on days 23 and 25 , fell outside of the two standard deviation band but were not consecutive and indicated no statistically significant change in the number of awakenings for phase B.

Comparison of sleep diary and Fitbit data. Data were collected simultaneously throughout the study using both the Consensus Sleep Diary and the Fitbit Inspire HR for each of the sleep metrics measured in the study and in each phase of the study. Figure 49 depicts a graphical comparison of these data for number of awakenings for participant two. 


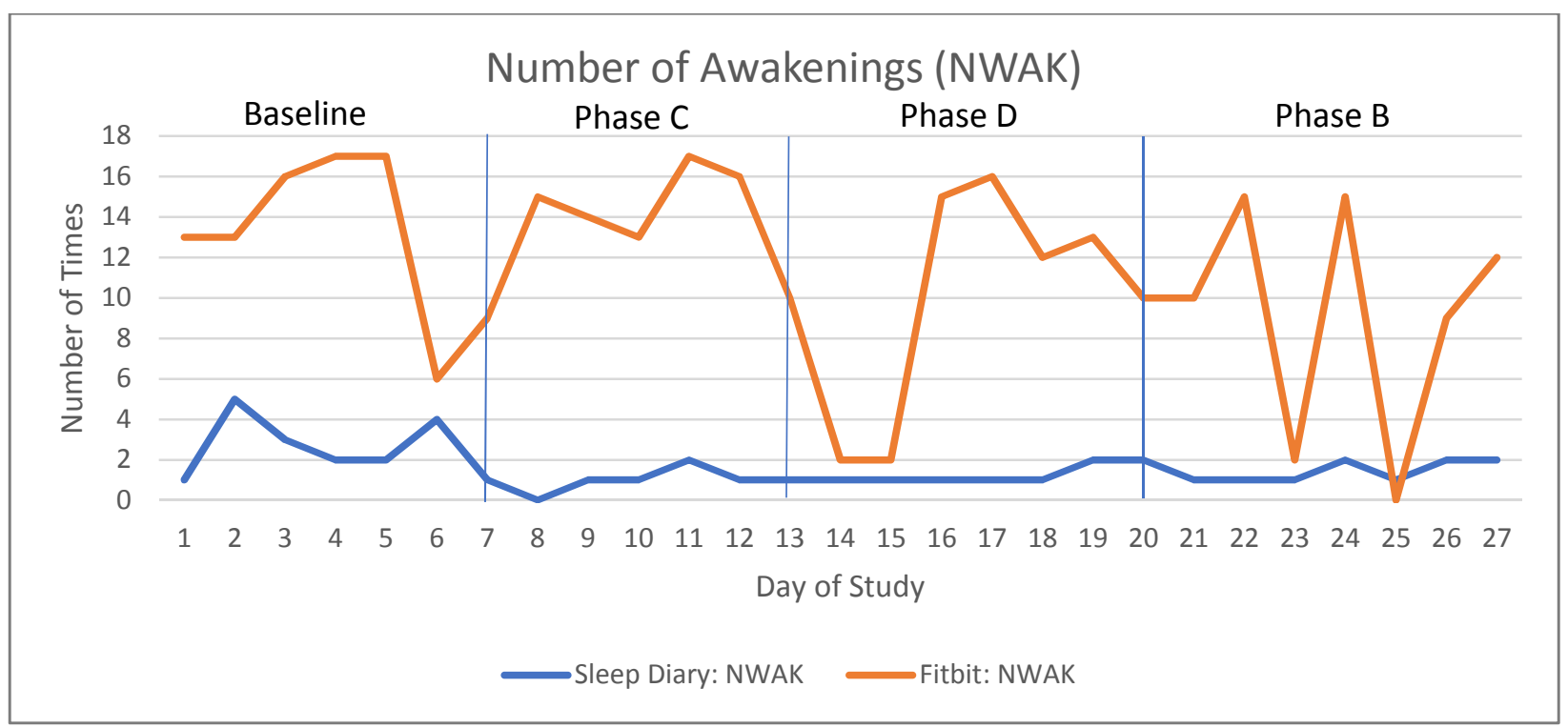

Figure 49. Participant Two Combined Data Number of Awakenings.

When comparing data for number of awakenings from the sleep diary and the Fitbit, there was no consistent trend between the two outcome measures. However, both the sleep diary and the Fitbit did show an overall decrease in the mean number of awakenings when compared to the mean of the baseline phase as the study progressed. Variability of the data was most similar between the outcome measures in phase C. See Table 3 for a comparison of the means of each phase for all variables for the sleep diary and the Fitbit device for participant two. 
Table 3

Participant Two Mean Comparison

\begin{tabular}{|c|c|c|c|c|c|c|c|c|c|c|c|c|c|c|c|c|}
\hline & \multicolumn{3}{|c|}{ Sleep Onset Latency } & \multicolumn{4}{|c|}{ Total Sleep Time } & \multicolumn{4}{|c|}{ Sleep Efficiency } & \multicolumn{5}{|c|}{ Number of Awakenings } \\
\hline & Baseline & Phase C & Phase D & Phase B & Baseline & Phase C & Phase D & Phase B & Baseline & Phase C & Phase D & Phase B & Baseline & Phase C & Phase D & Phase B \\
\hline Diary & 15.71 & 21.00 & 15.71 & 8.86 & 412.86 & 438.17 & 403.71 & 497.51 & 79.943 & 83.583 & 86.871 & 92.100 & 2.57 & 1.00 & 1.29 & 1.43 \\
\hline Fitbit & 4.14 & 1.17 & 19.86 & 9.86 & 390.00 & 400.83 & 423.57 & 391.71 & 75.571 & 76.800 & 89.729 & 72.129 & 13.0 & 14.17 & 10.0 & 9.0 \\
\hline
\end{tabular}




\section{Severity of Insomnia}

A participant's level of insomnia was measured at the beginning of the study, after all interventions were completed, and at a 1-month follow-up after interventions were completed. The severity of insomnia was measured using the Insomnia Severity Index, with lower scores indicating less insomnia. Figure 50 shows the Insomnia Severity Index assessment results for participant two.

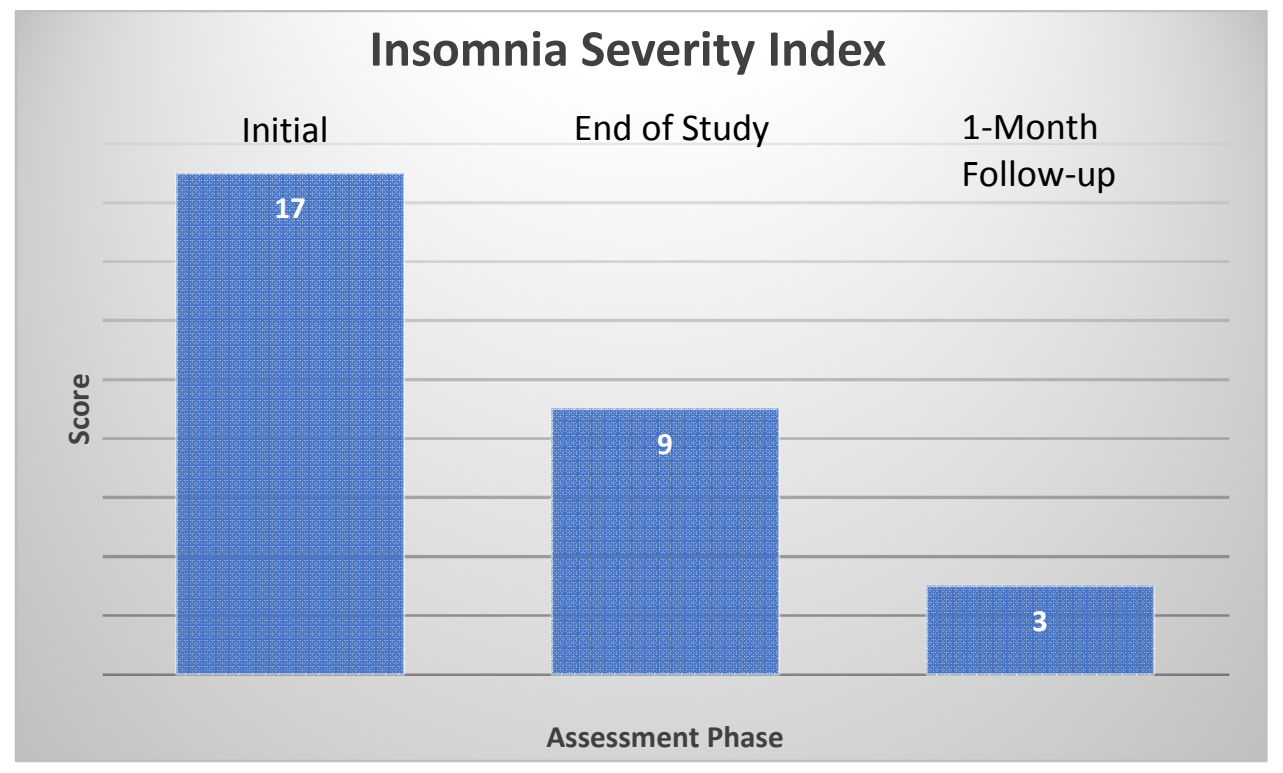

Figure 50. Participant Two Insomnia Severity Index.

Daniel's initial Insomnia Severity Index score fell within the "moderate insomnia range" (Morin, 1993). After completing the study interventions, his Insomnia Severity Score fell within the "sub-threshold insomnia range" (Morin, 1993), indicating a reduction in the severity of his insomnia that was still clinically significant. At his one-month follow-up, his Insomnia Severity Index Score declined even further, indicating no clinically significant insomnia one month after participating in the sleep interventions. 


\section{Functional Outcomes of Sleep}

To determine the functional implications of disruptions in sleep, the Functional Outcomes of Sleep Questionnaire was administered at the beginning of the study and after the completion of each intervention phase. Higher scores indicated less disruption in functional outcomes. Figure 51 shows the results of the Functional Outcomes of Sleep Questionnaire assessments for participant two.

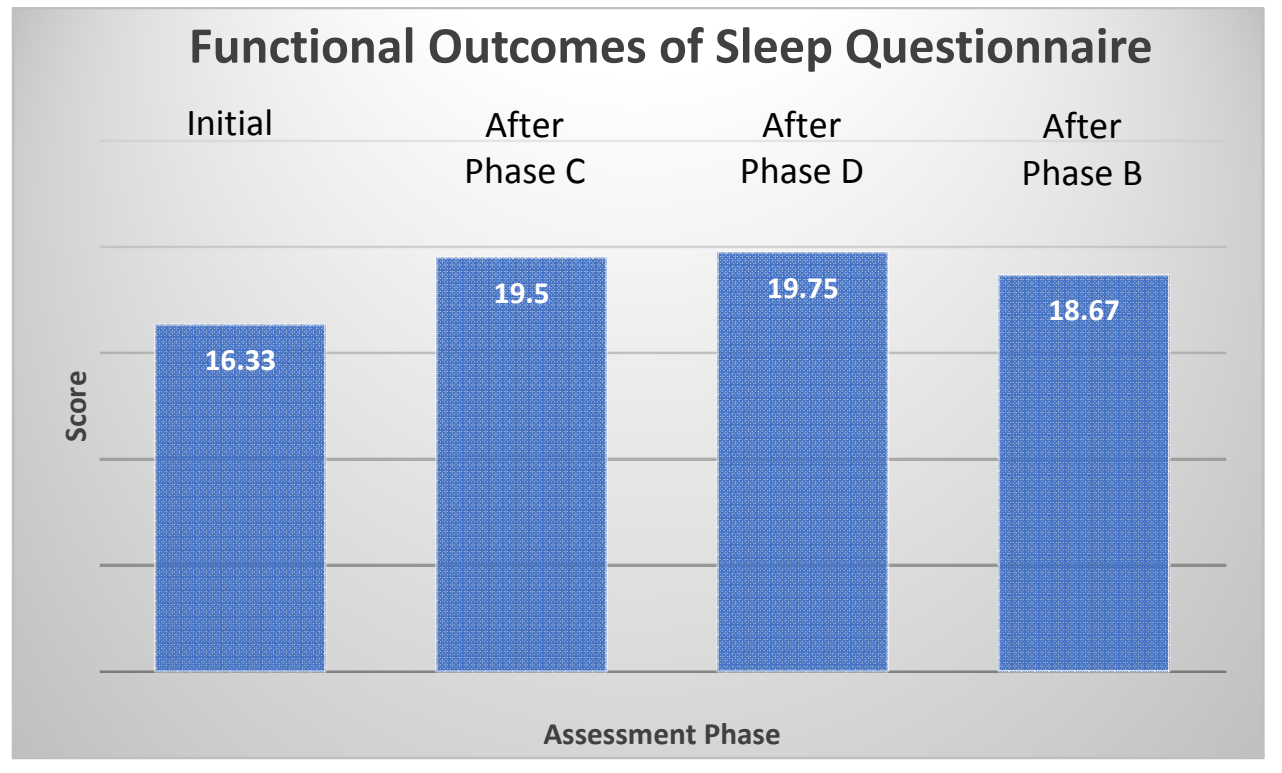

Figure 51. Participant Two Functional Outcomes of Sleep Questionnaire.

The results of Daniel's Functional Outcomes of Sleep Questionnaire assessments indicated that he had less disruptions in functional outcomes after each of the intervention phase when compared to his initial assessment. There was a slight increase in functional disruptions after participating in the last intervention phase of the study, phase B.

\section{Sleepiness}

To determine the level of sleepiness of participants while participating in the dissertation study, the Epworth Sleepiness Scale was administered at the beginning of the study and after the 
completion of each phase of the study. Lower scores indicated less sleepiness. Figure 52 shows the results of the Epworth Sleepiness Scale assessments for participant two.

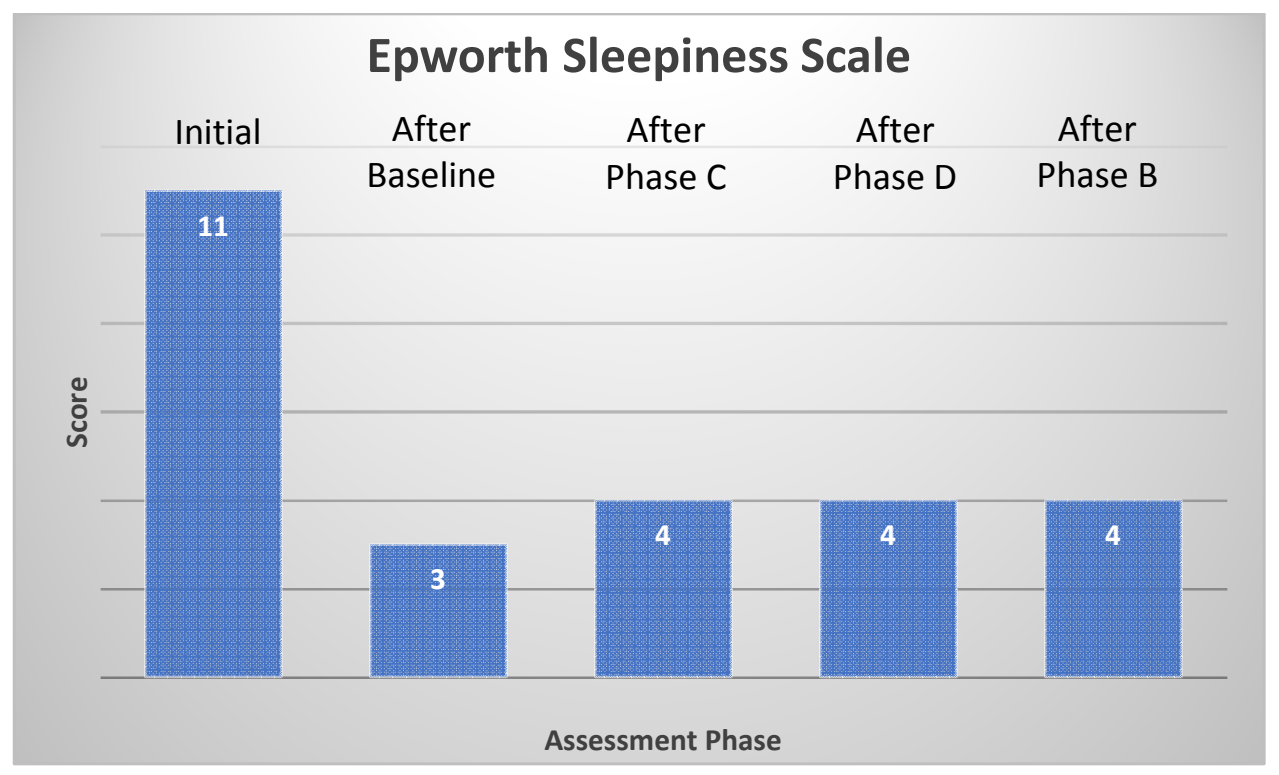

Figure 52. Participant Two Epworth Sleepiness Scale.

The results of Daniel's Epworth Sleepiness Scale assessments indicated a decrease in sleepiness after participating in the intervention phases as compared to his initial Epworth Sleepiness Scale score. His initial Epworth Sleepiness Scale score of 11 indicated "mild excessive daytime sleepiness," but the decline after the initial assessment indicated "lower normal daytime sleepiness" (Johns, 1991) throughout the baseline and intervention phases.

\section{Participant Three - Keith}

Keith initiated participation in the sleep program in December 2019 and completed the program 4 weeks later. When completing his sleep history forms, Keith indicated occasional difficulty with staying asleep, occasional worrying in bed, occasionally having inconsistent bedtimes and wake times, and occasionally using the bed for activities other than sleep or sexual activity. Baseline data for the sleep program were collected for 7 days, and baseline stability was achieved during this time. For Keith, the study phases occurred in the order of baseline data 
collection, intervention phase $\mathrm{D}$, intervention phase $\mathrm{B}$, and then intervention phase $\mathrm{C}$. Data were collected in all phases of the study using both the sleep diary and the Fitbit device. Sleep metrics measured in all phases included sleep onset latency, total sleep time, sleep efficiency, and number of awakenings.

\section{Sleep Onset Latency}

Sleep onset latency is graphically depicted in Figure 53 for sleep diary data and in Figure 54 for Fitbit data.

Consensus Sleep Diary. Figure 53 depicts sleep onset latency for all phases of the study using sleep diary data for participant three.

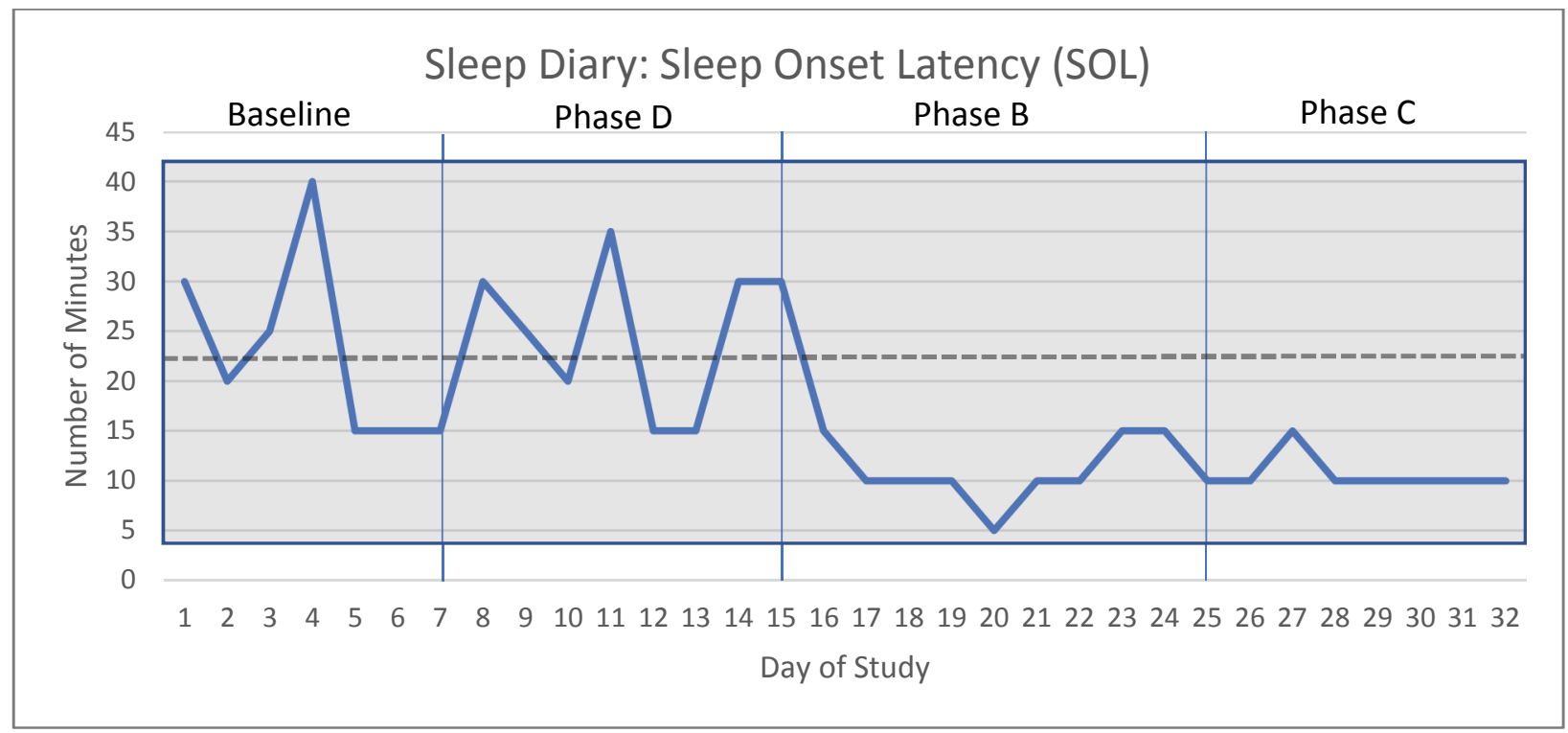

Figure 53. Participant Three Sleep Diary Sleep Onset Latency. Dashed lines represent the mean of the baseline phase. The gray squared box represents the Two Standard Deviation Band.

Using visual analysis techniques of the graph in Figure 53, the level, trend, slope, and variability were calculated for sleep onset latency from the sleep diary data.

Level. Level was determined by comparing the mean values of sleep onset latency within each phase. The mean value for sleep onset latency of the baseline phase is depicted in Figure 
53, which was 22.86. The mean for phase D was 25.0, phase B was 11.0, and for phase C 10.71 . This indicates that the average time for sleep onset latency increased for the first intervention phase in phase D but decreased for next two intervention phases for phases B and C.

Trend. Data for sleep onset latency showed no trend for baseline data and intervention phases D and C and a decelerating trend for intervention phase B. The overall trend of data was decelerating from baseline to the end of the study, indicating an overall decrease in sleep onset latency as the study progressed. The direction of change was curvilinear in phase B, indicating more consistent changes in that phase.

Variability. The direction of change was variable throughout the phases with less variable noted in phase $B$ and relatively stable data in phase $\mathrm{C}$.

Inferential statistics. Using the two standard deviation band method, no data points fell outside of the two standard deviation band. This indicated no statistically significant change in sleep onset latency.

Fitbit Inspire HR. Figure 54 depicts sleep onset latency for all phases of the study using Fitbit data for participant three. Using visual analysis techniques of the graph in Figure 54, the level, trend, slope, and variability were calculated for sleep onset latency from the Fitbit data.

Level. The mean value for sleep onset latency of the baseline phase is depicted in Figure 54, which was 10.86 . The mean for phase $\mathrm{D}$ was 24.75 , phase $\mathrm{B}$ was 38.40 , and for phase C 31.29. This indicates that the average time for sleep onset latency increased for each of the intervention phases when compared to the baseline.

Trend. Data for sleep onset latency showed no trend for intervention phases B and C and mild decelerating trend in baseline data and in intervention phases $\mathrm{D}$, indicating fluctuations in sleep onset latency throughout the study with no significant changes noted for sleep efficiency 
from baseline to intervention phases. The direction of change was variable throughout the phases and was curvilinear in the baseline phase and in intervention phase D.

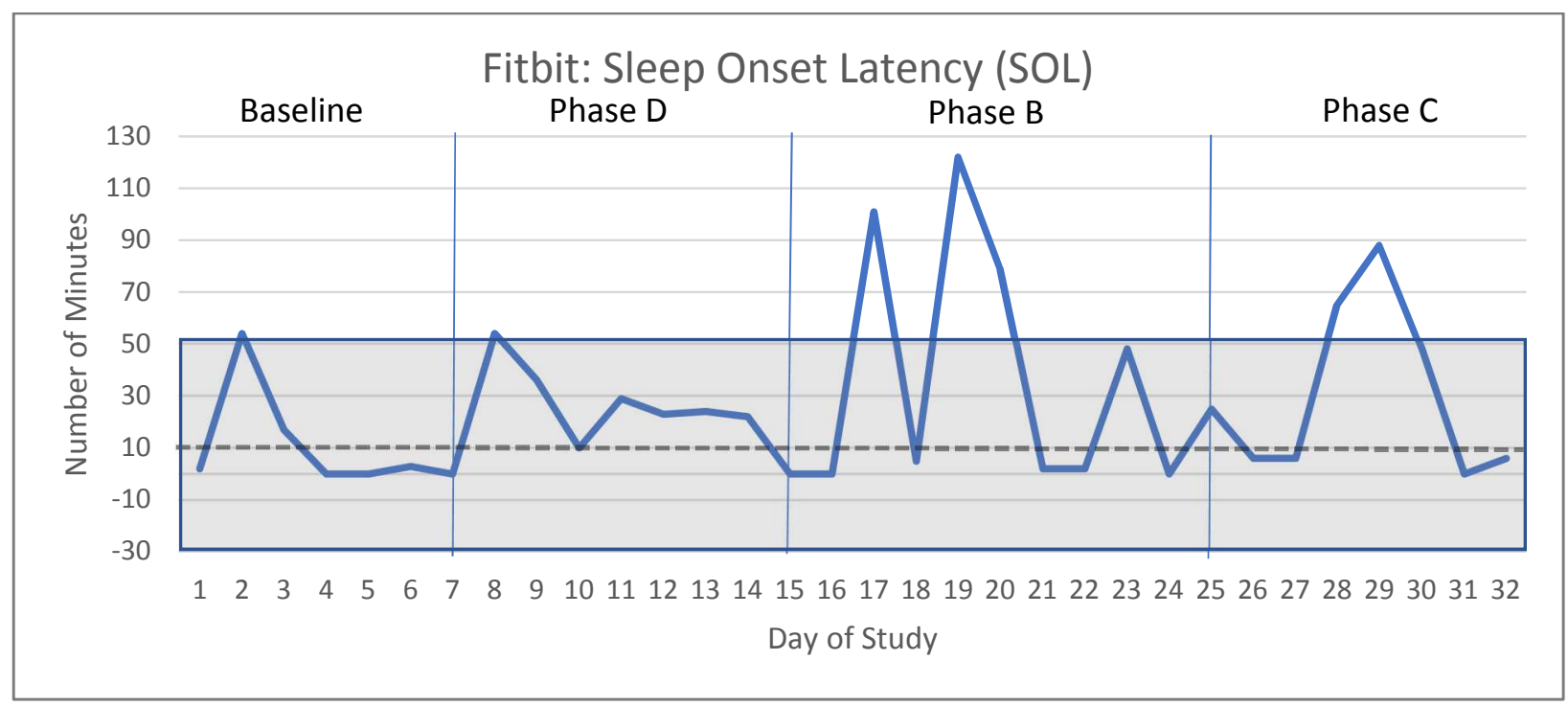

Figure 54. Participant Three Fitbit Sleep Onset Latency. Dashed lines represent the mean of the baseline phase. The gray squared box represents the Two Standard Deviation Band.

Variability. The direction of change was variable throughout the phases with less variability noted in the baseline phase and in intervention phase $\mathrm{D}$, indicating more stable data in those phases.

Inferential statistics. Using the two standard deviation band method, data points on days 17, 19, and 20 fell outside of the two standard deviation band during phase B. Data points on days 28 and 29 in phase $\mathrm{C}$ also fell outside of the two standard deviation band, indicating a statistically significant change in sleep onset latency during phases B and C.

Comparison of sleep diary and Fitbit data. Data were collected simultaneously throughout the study using both the Consensus Sleep Diary and the Fitbit Inspire HR for each of the sleep metrics measured in the study and in each phase of the study. Figure 55 depicts a graphical comparison of these data for sleep onset latency for participant three. 


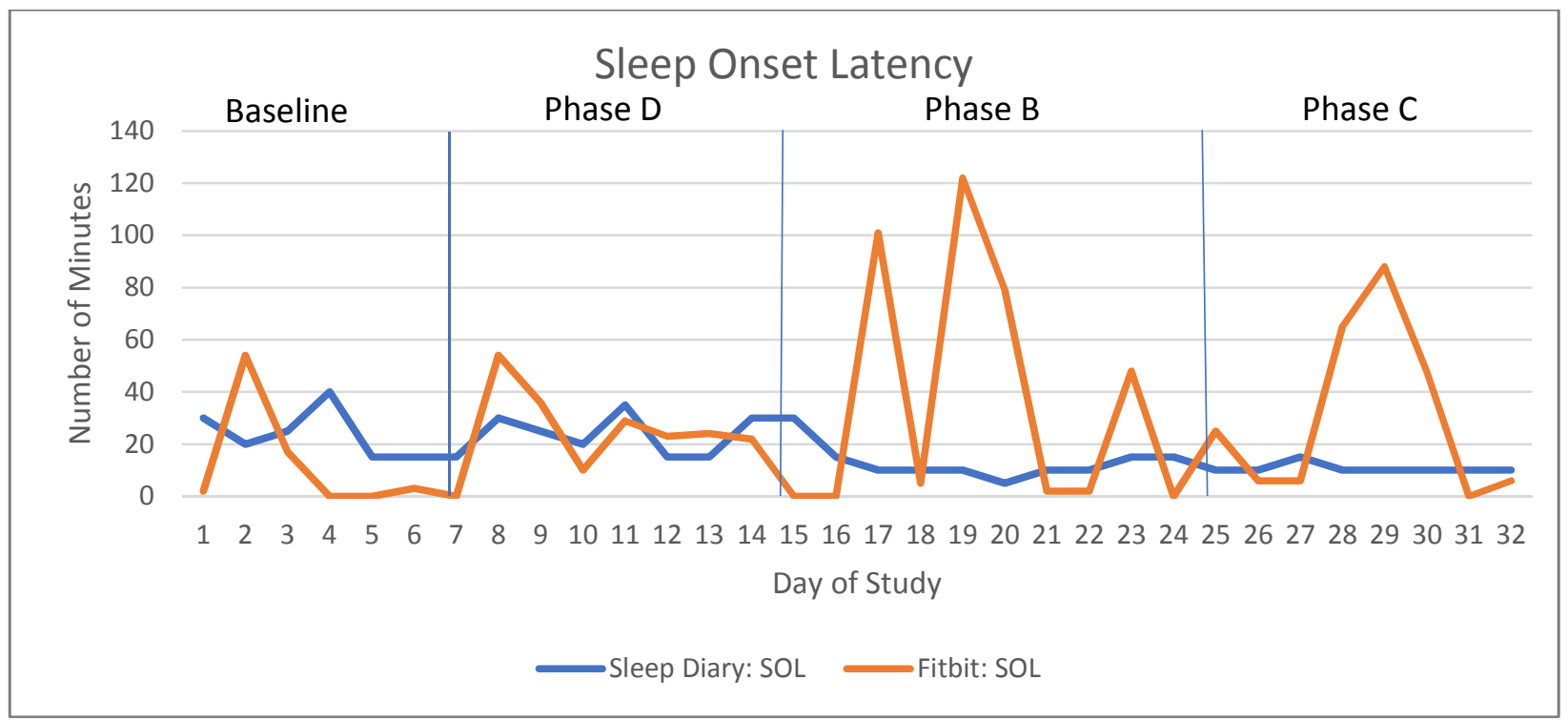

Figure 55. Participant Three Combined Data Sleep Onset Latency.

When comparing data for sleep onset latency from the sleep diary and the Fitbit, there was no consistent trend between the two outcome measures. Sleep diary data showed an overall decrease in sleep onset latency as the study progressed and the Fitbit data showed an increase in sleep onset latency when comparing data to the baseline phase.

\section{Total Sleep Time}

Total sleep time is graphically depicted in Figure 56 for sleep diary data and in Figure 61 for Fitbit data.

Consensus Sleep Diary. Figure 56 depicts total sleep time for all phases of the study using sleep diary data for participant three.

Level. The mean value for total sleep time of the baseline phase is depicted in Figure 56, which was 401.43. The mean for phase D was 455.0, phase B was 511.0, and for phase C 517.14. This indicates that the average total sleep time progressively increased throughout the intervention phases. 


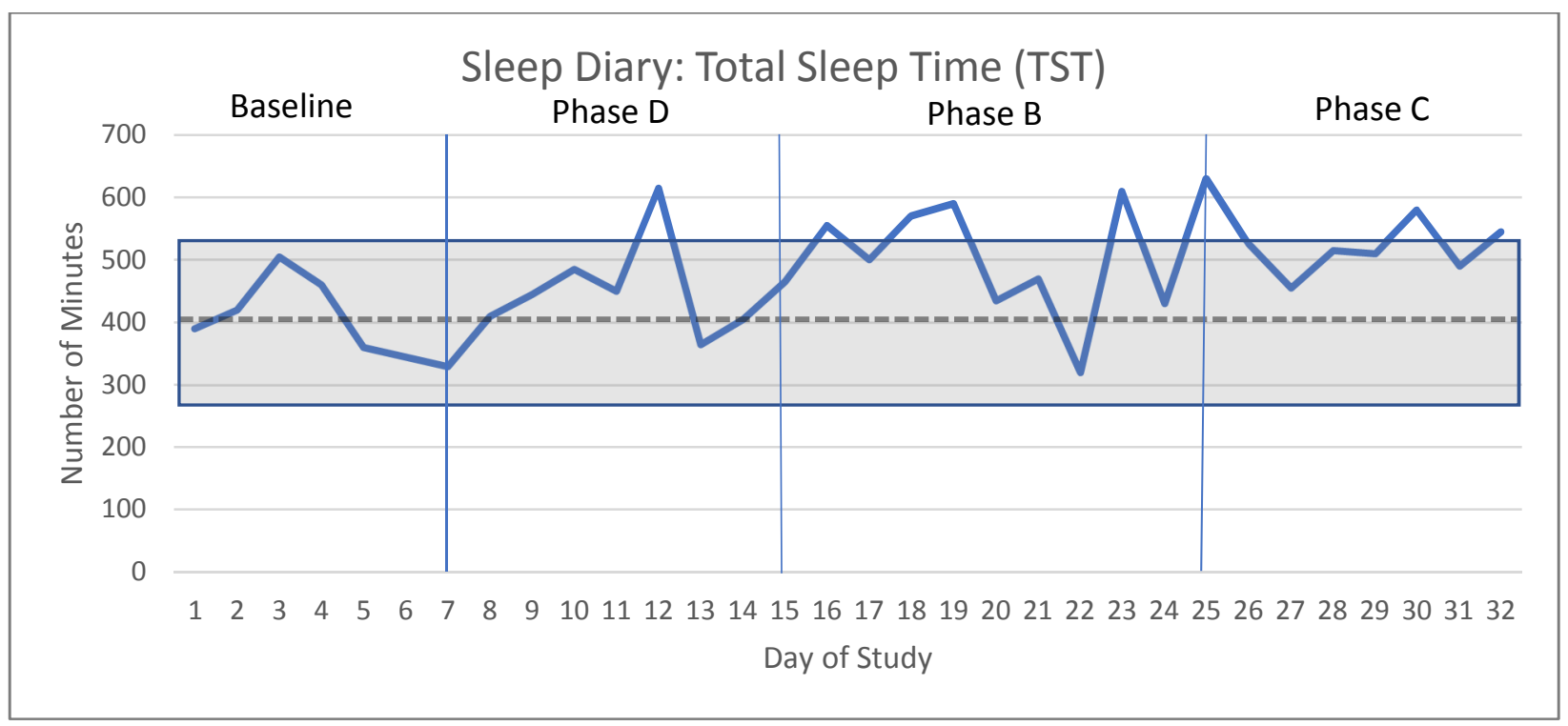

Figure 56. Participant Three Sleep Diary Total Sleep Time. Dashed lines represent the mean of the baseline phase. The gray squared box represents the Two Standard Deviation Band.

Using visual analysis techniques of the graph in Figure 56, the level, trend, slope, and variability were calculated for total sleep time from the sleep diary data.

Trend. Data for total sleep time showed no trend for baseline data and intervention phase. However, the overall trend of data from baseline to the end of the study was accelerating, indicating an overall increase in total sleep time as the study progressed. The direction of change was curvilinear in the baseline phase. A celeration line was calculated for total sleep time using the sleep diary data and is graphically depicted in Figure 57. Each of the data points in all of the intervention phases fell above the celeration line, indicating a significant change in Keith's response pattern during the intervention phases when compared to the baseline phase. 


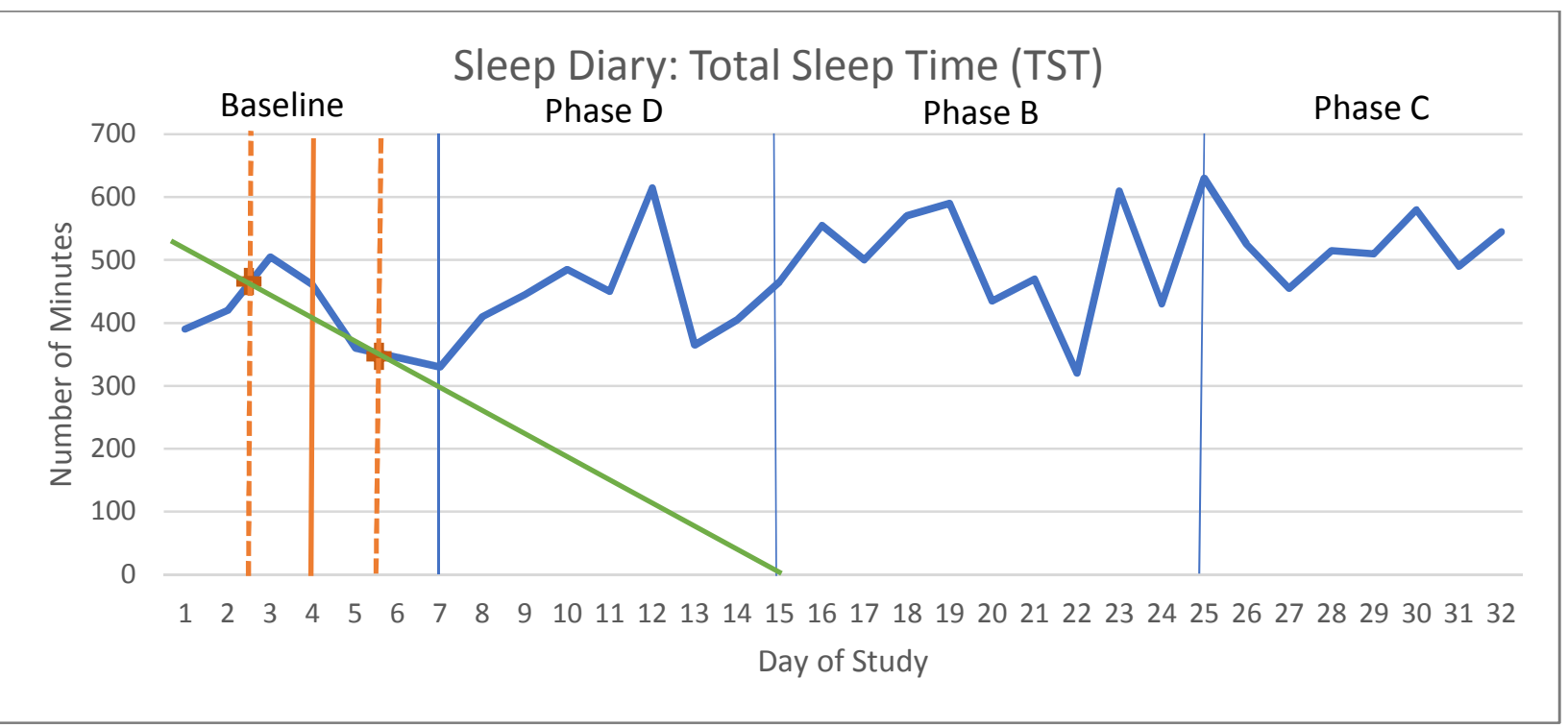

Figure 57. Participant Three Total Sleep Time Celeration Line. The solid orange line represents a dividing line for half of the baseline data. Dashed orange lines represent a dividing line for quarters of the baseline data. The solid green line represents the celeration line.

Slope. The slope of the trend line was calculated for each of the phases to show the change in the angle of the trend. A comparison between the intervention phases was done by calculating the slope for each phase individually. The slope of the trend line was calculated for each of the phases to show the change in the angle of the trend. The slope of data in phase D was 1.07 and decelerating, indicating that total sleep time decreased at an average rate of 1.07 times per week during this phase. The slope of data in phase B was 1.34 decelerating, indicating that total sleep time decreased at an average rate of 1.34 times per week during this phase. The slope of data in phase $\mathrm{C}$ was 1.05 and decelerating, indicating that total sleep time decreased at an average rate of 1.05 times per week during this phase. Decelerating slopes for each intervention phase indicated decreases in total sleep time in each intervention phase, with the most significant rate of change noted in intervention phase B. The slope of sleep efficiency data for each intervention phase is visually depicted in Figures 58, 59, and 60. 


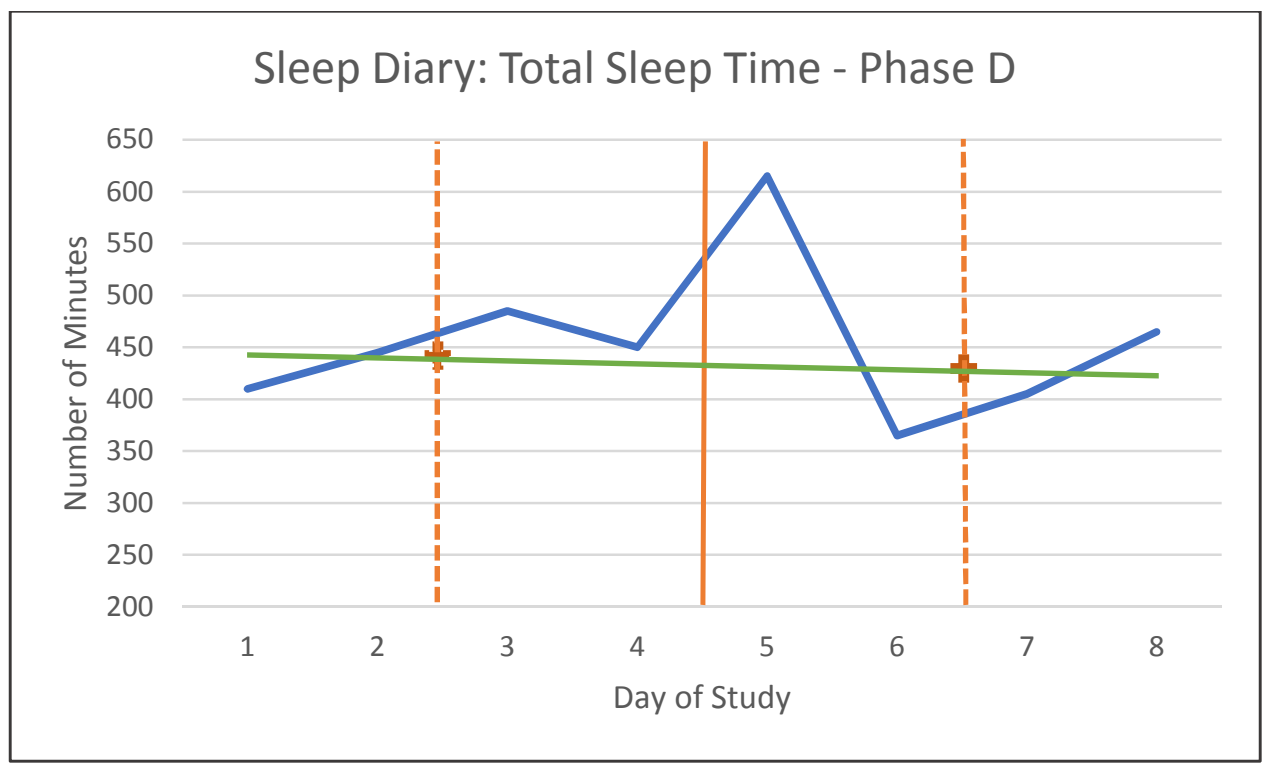

Figure 58. Participant Three Sleep Diary Total Sleep Time Slope Phase D. The solid orange line represents a dividing line for half of the baseline data. Dashed orange lines represent a dividing line for quarters of the baseline data. The solid green line represents the celeration line.

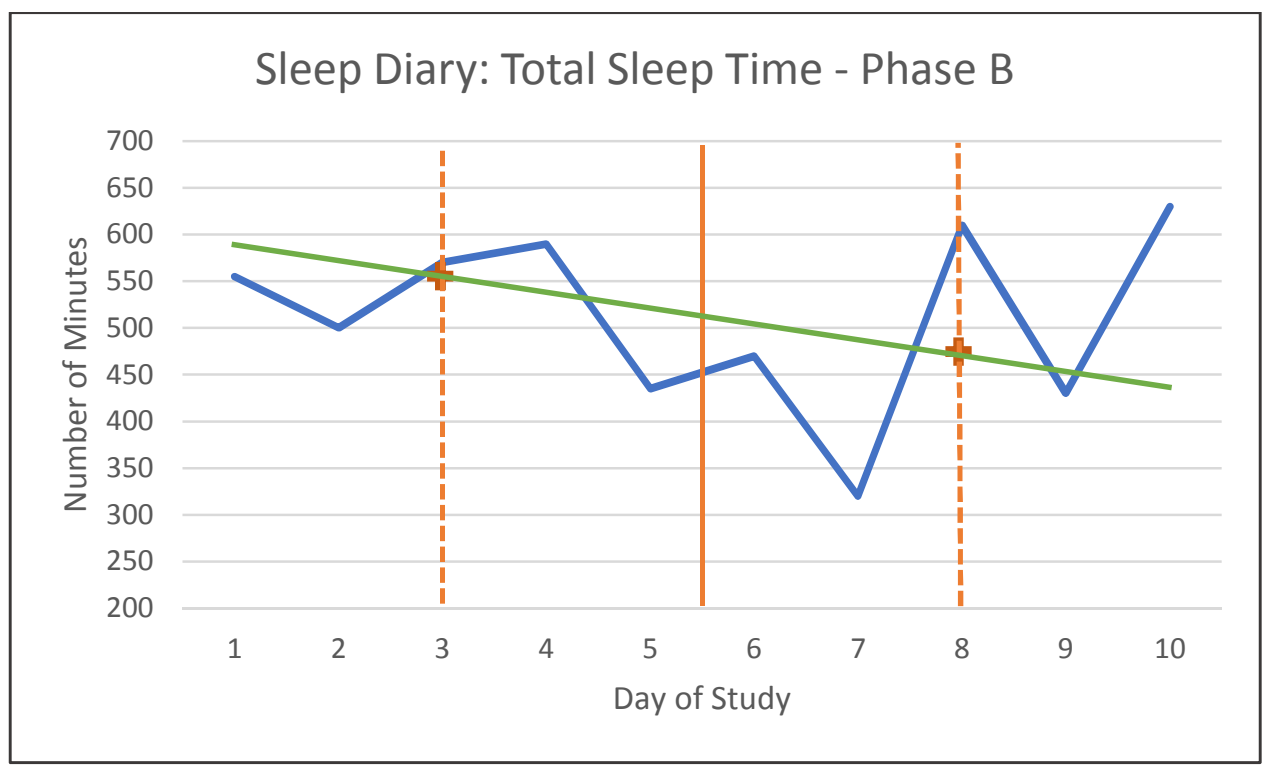

Figure 59. Participant Three Sleep Diary Total Sleep Time Slope Phase B. The solid orange line represents a dividing line for half of the baseline data. Dashed orange lines represent a dividing line for quarters of the baseline data. The solid green line represents the celeration line. 


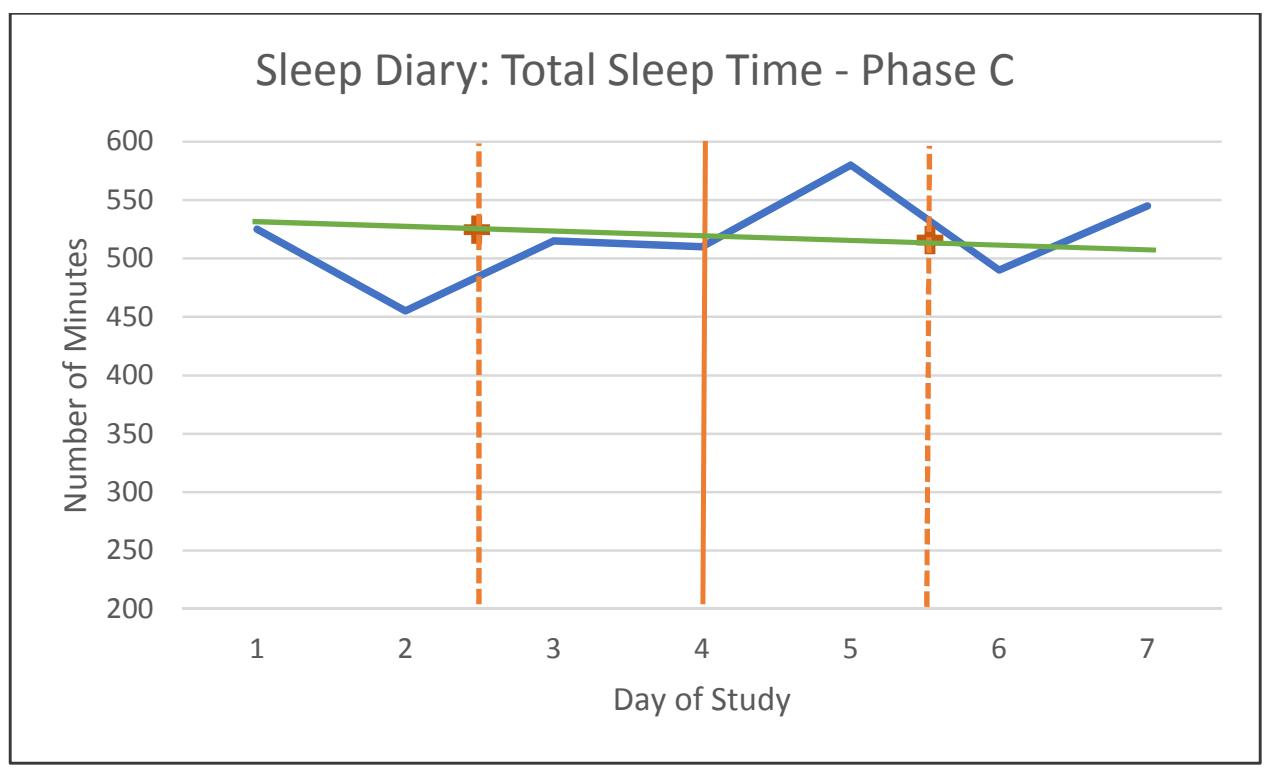

Figure 60. Participant Three Sleep Diary Total Sleep Time Slope Phase C. The solid orange line represents a dividing line for half of the baseline data. Dashed orange lines represent a dividing line for quarters of the baseline data. The solid green line represents the celeration line.

Variability. The direction of change was variable throughout the phases with less variability in the baseline phase, indicating more stable data in that phase.

Inferential statistics. Using the two standard deviation band method, one data point in phase D, five out of seven data points in phase B, and two data points in phase C fell outside of the two standard deviation band. There was a statistically significant increase in total sleep time in phase $\mathrm{B}$. The $\mathrm{C}$ statistic was calculated for the baseline phase, which yielded $\mathrm{C}=0.582$ with a standard error of 0.3227 . The $\mathrm{z}$ score was $1.804(1.804<1.64)$, indicating a significant trend in the baseline data and making it more difficult to assess a significant trend across baseline and intervention phases. Therefore, additional c-statistic calculations were not completed for this variable.

Fitbit Inspire HR. Figure 61 depicts total sleep time for all phases of the study using Fitbit data for participant three. 


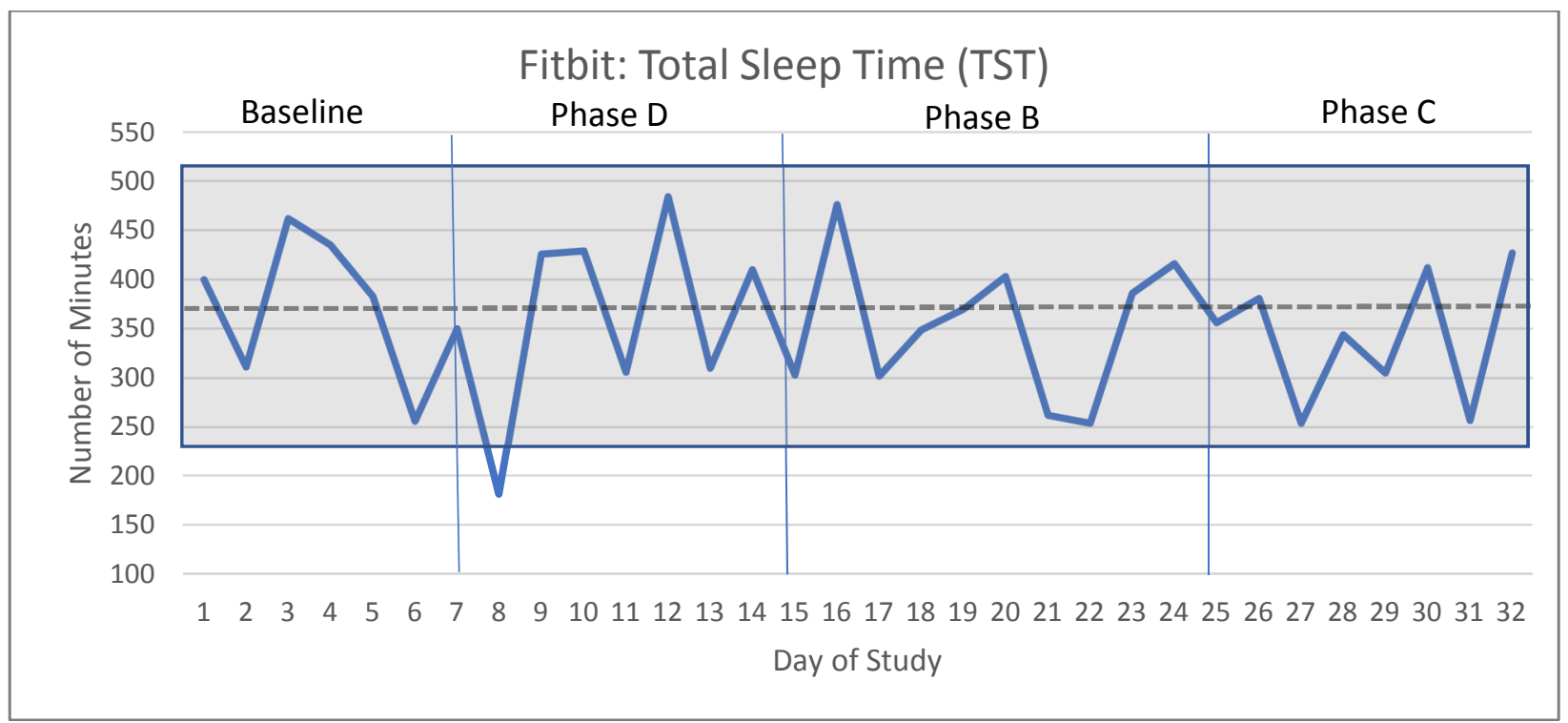

Figure 61. Participant Three Fitbit Total Sleep Time. Dashed lines represent the mean of the baseline phase. The gray squared box represents the Two Standard Deviation Band.

Using visual analysis techniques of the graph in Figure 61, the level, trend, slope, and variability were calculated for total sleep time from the Fitbit data.

Level. The mean value for total sleep time of the baseline phase is depicted in Figure 61, which was 371.0. The mean for phase $\mathrm{D}$ was 356.13 , phase $\mathrm{B}$ was 357.40 , and for phase $\mathrm{C}$ 340.0. This indicated that the average total sleep time slightly decreased for each of the intervention phases.

Trend. Data for total sleep time showed no trend for baseline data and intervention phases, indicating fluctuations in total sleep time throughout the study with no consistent change noted from baseline to intervention phases.

Variability. The direction of change was variable throughout the phases with minimal stability noted in the data. 
Inferential statistics. Using the two standard deviation band method, only one data point fell outside of the two standard deviation band. This indicated no statistically significant change in total sleep time.

Comparison of sleep diary and Fitbit data. Data were collected simultaneously throughout the study using both the Consensus Sleep Diary and the Fitbit Inspire HR for each of the sleep metrics measured in the study and in each phase of the study. Figure 62 depicts a graphical comparison of these data for total sleep time for participant three.

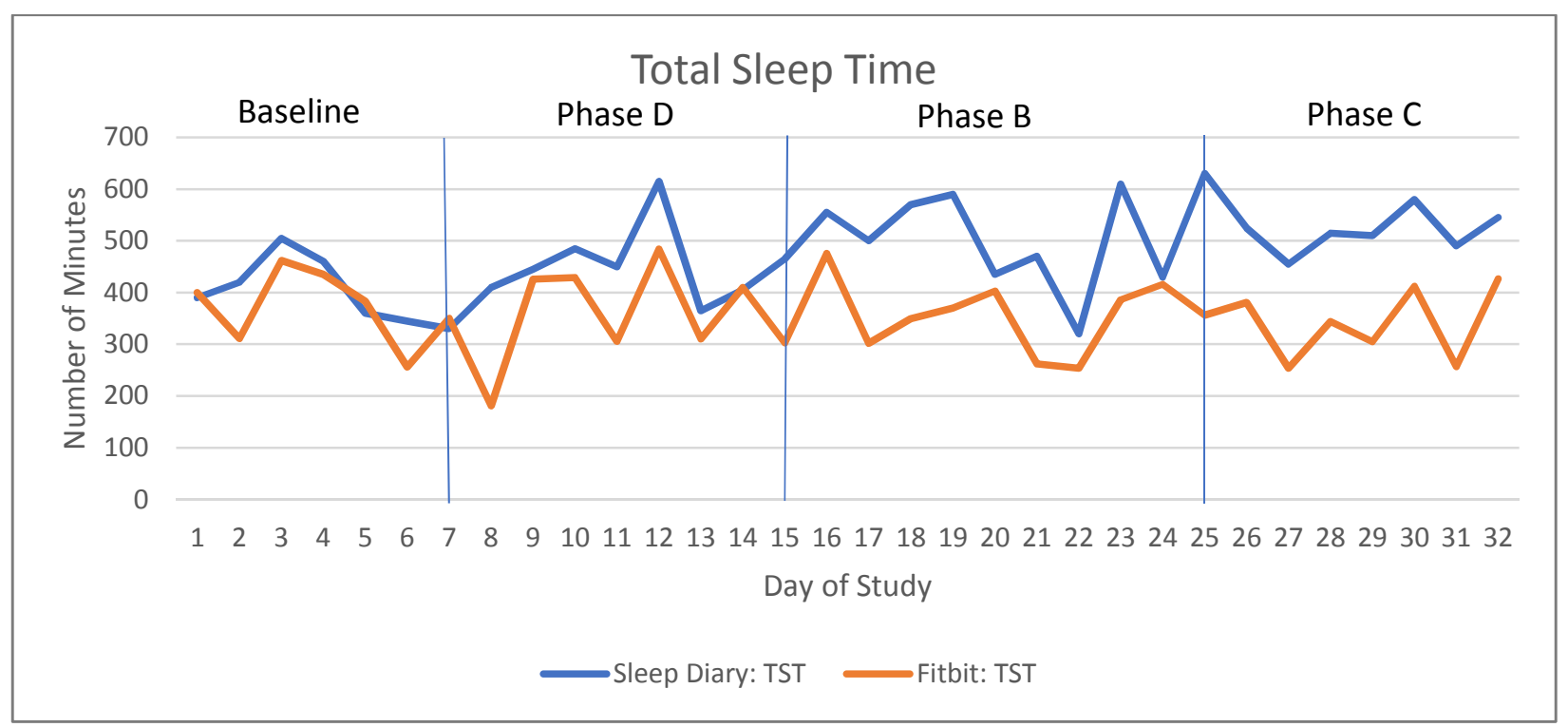

Figure 62. Participant Three Combined Data Total Sleep Time.

When comparing data for total sleep time from the sleep diary and the Fitbit, there was no consistent trend between the two outcome measures. Variability in data was most similar in intervention phase B of the study.

\section{Sleep Efficiency}

Sleep efficiency is graphically depicted in Figure 63 for sleep diary data and in Figure 68 for Fitbit data. 
Consensus Sleep Diary. Figure 63 depicts sleep efficiency for all phases of the study using sleep diary data for participant three.

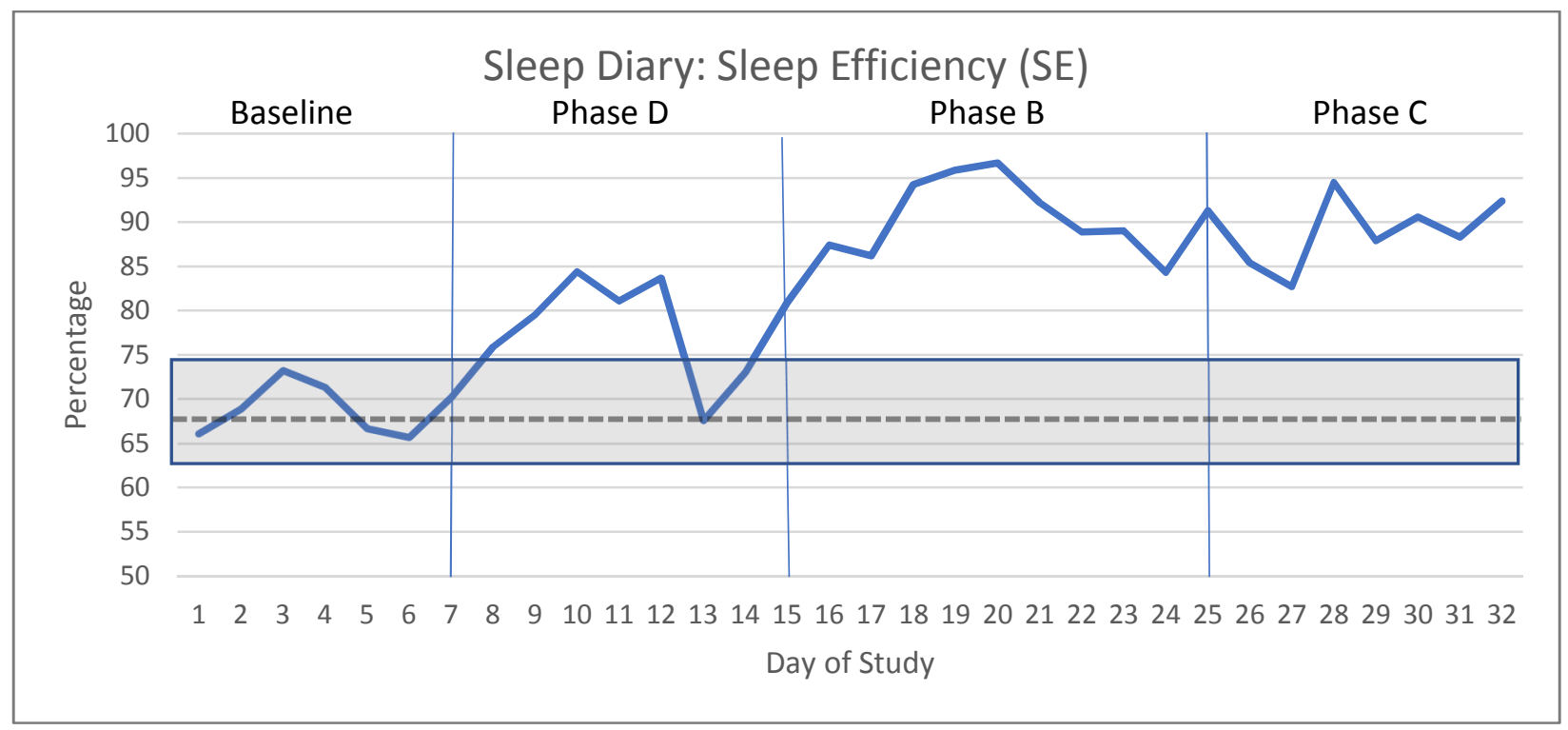

Figure 63. Participant Three Sleep Diary Sleep Efficiency. Dashed lines represent the mean of the baseline phase. The gray squared box represents the Two Standard Deviation Band.

Using visual analysis techniques of the graph in Figure 63, the level, trend, slope, and variability were calculated for sleep efficiency from the sleep diary data.

Level. The mean value for sleep efficiency of the baseline phase is depicted in Figure 63, which was 68.871 . The mean for phase $\mathrm{D}$ was 78.263 , phase $\mathrm{B}$ was 90.610 , and for phase $\mathrm{C}$ 88.829. This indicated that the average sleep efficiency increased for all intervention phases when compared to the baseline data, with the most improvement noted in phase B.

Trend. Data for sleep efficiency showed no trend within phases for baseline data and intervention phases. However, the overall trend of data was accelerating from baseline to the end of the study, indicating a significant increase in sleep efficiency as the study progressed. The direction of change was variable throughout the phases. A celeration line was calculated for sleep efficiency using the sleep diary data and is graphically depicted in Figure 64. 


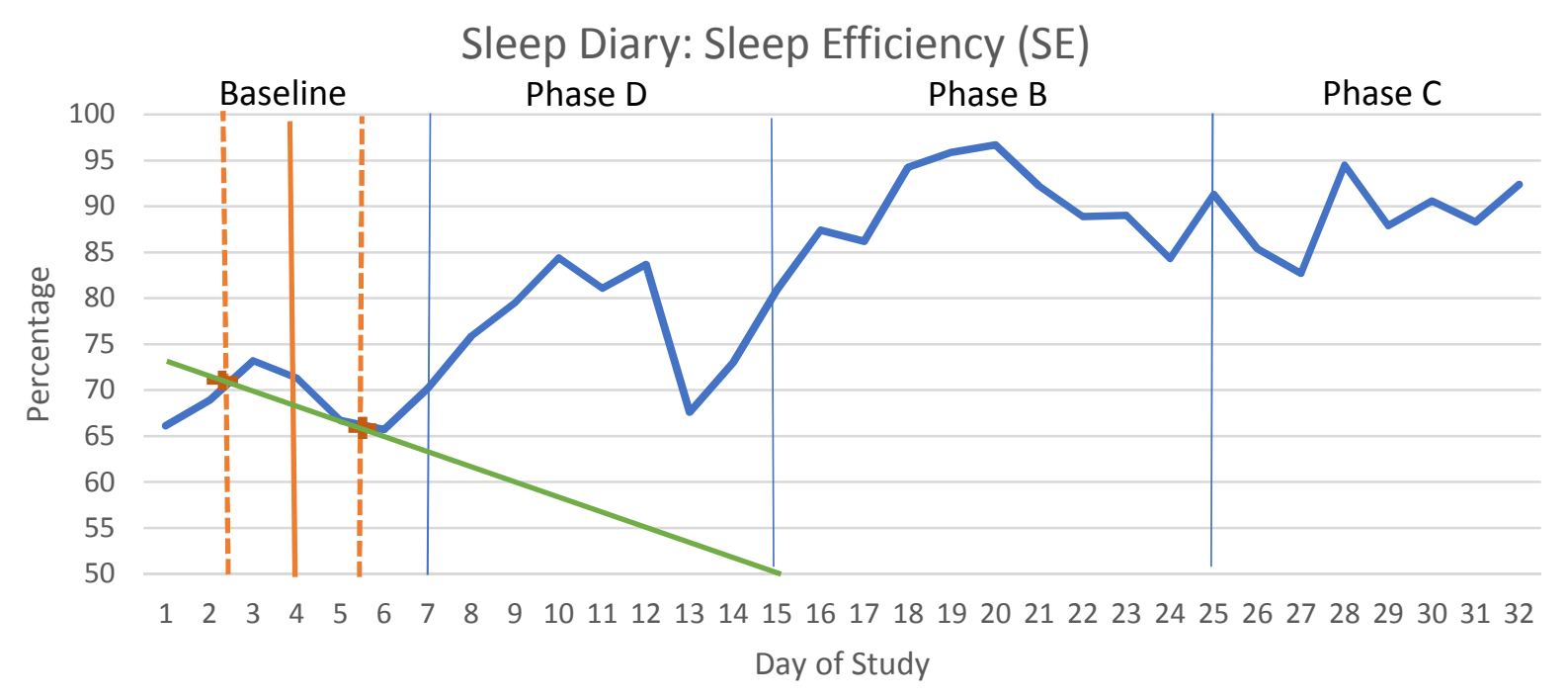

Figure 64. Participant Three Sleep Diary Sleep Efficiency Trend. The solid orange line represents a dividing line for half of the baseline data. Dashed orange lines represent a dividing line for quarters of the baseline data. The solid green line represents the celeration line.

Each of the data points in all of the intervention phases fell above the celeration line, indicating a significant change in Keith's response pattern during the intervention phases when compared to the baseline phase.

Slope. The slope of data in phase D was 1.1 and decelerating, indicating that sleep efficiency decreased at an average rate of 1.1 times per week during this phase. The slope of data in phase B was 1.13 and decelerating, indicating that sleep efficiency decreased at an average rate of 1.13 times per week during this phase. The slope of data in phase $\mathrm{C}$ was 1.0 , indicating that sleep efficiency changed at an average rate of 1.0 times per week during this phase. The slope of sleep efficiency data for each intervention phase is visually depicted in Figures 65, 66, and 67. 


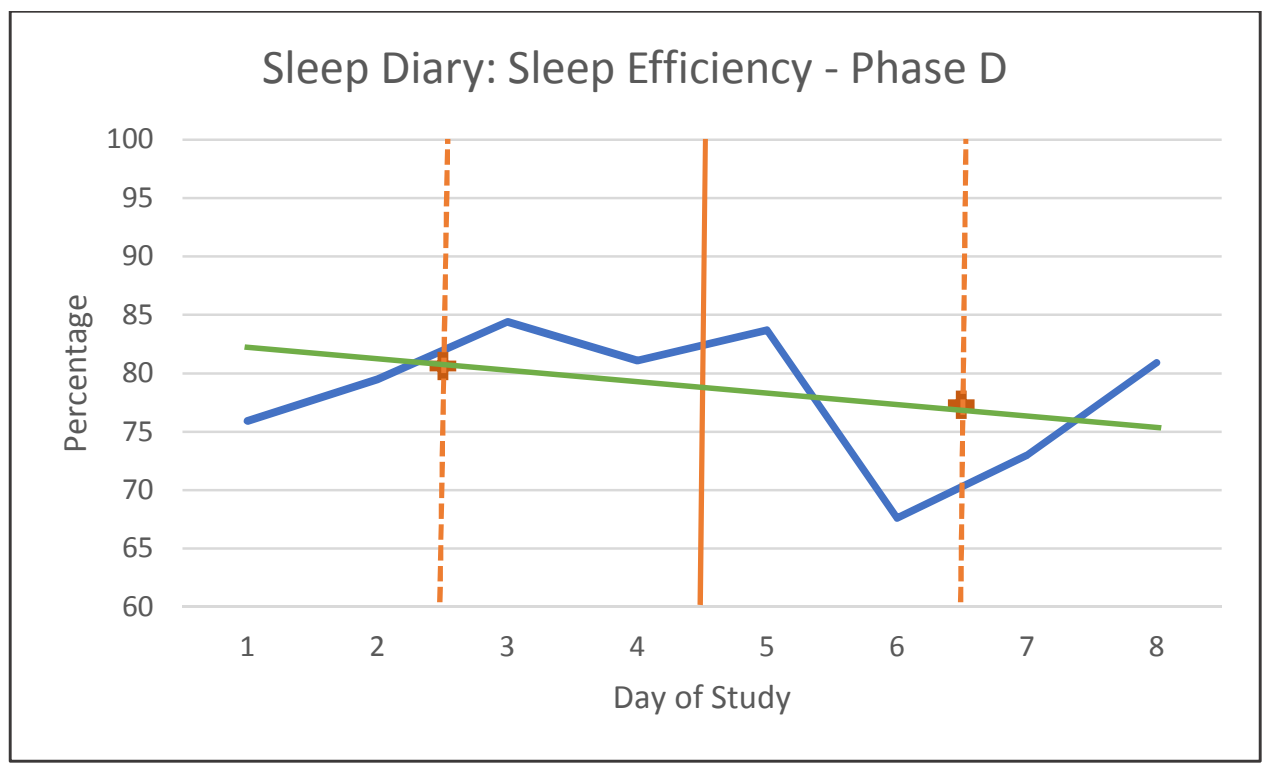

Figure 65. Participant Three Sleep Diary Sleep Efficiency Slope Phase D. The solid orange line represents a dividing line for half of the baseline data. Dashed orange lines represent a dividing line for quarters of the baseline data. The solid green line represents the celeration line.

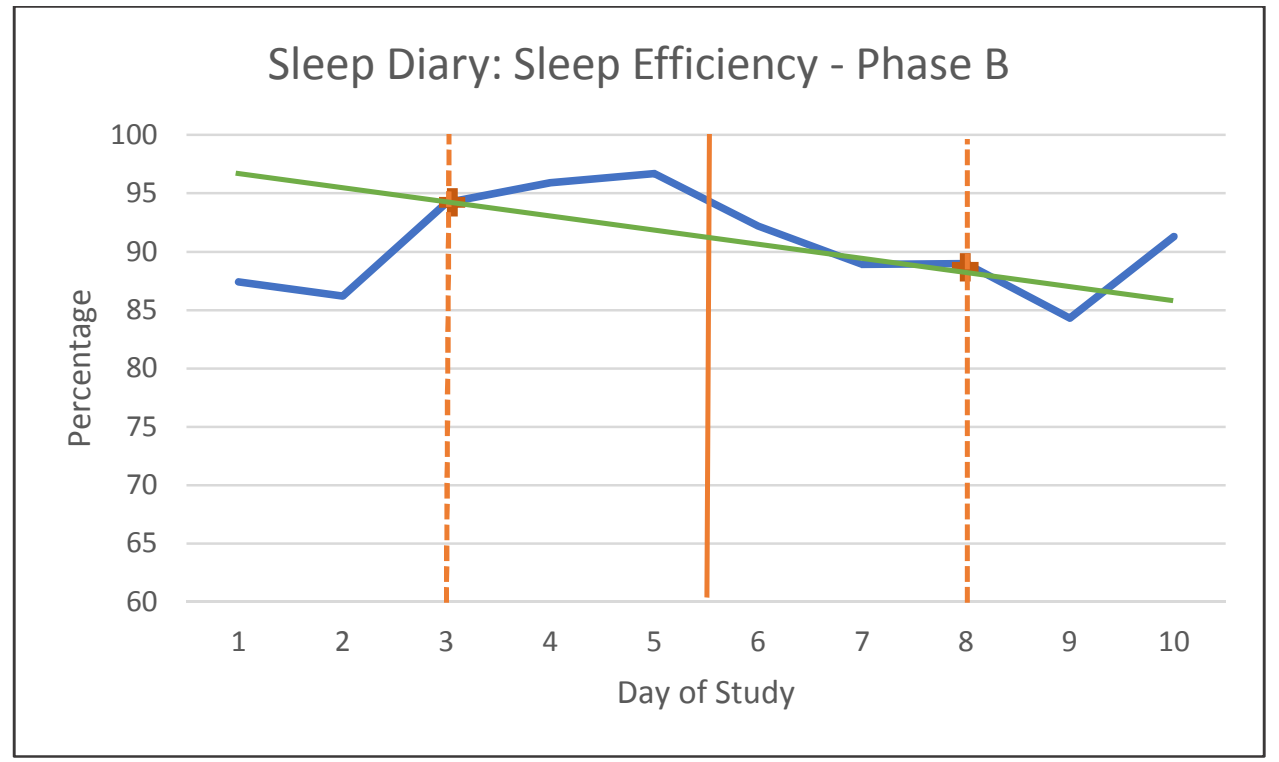

Figure 66. Participant Three Sleep Diary Sleep Efficiency Slope Phase B. The solid orange line represents a dividing line for half of the baseline data. Dashed orange lines represent a dividing line for quarters of the baseline data. The solid green line represents the celeration line. 


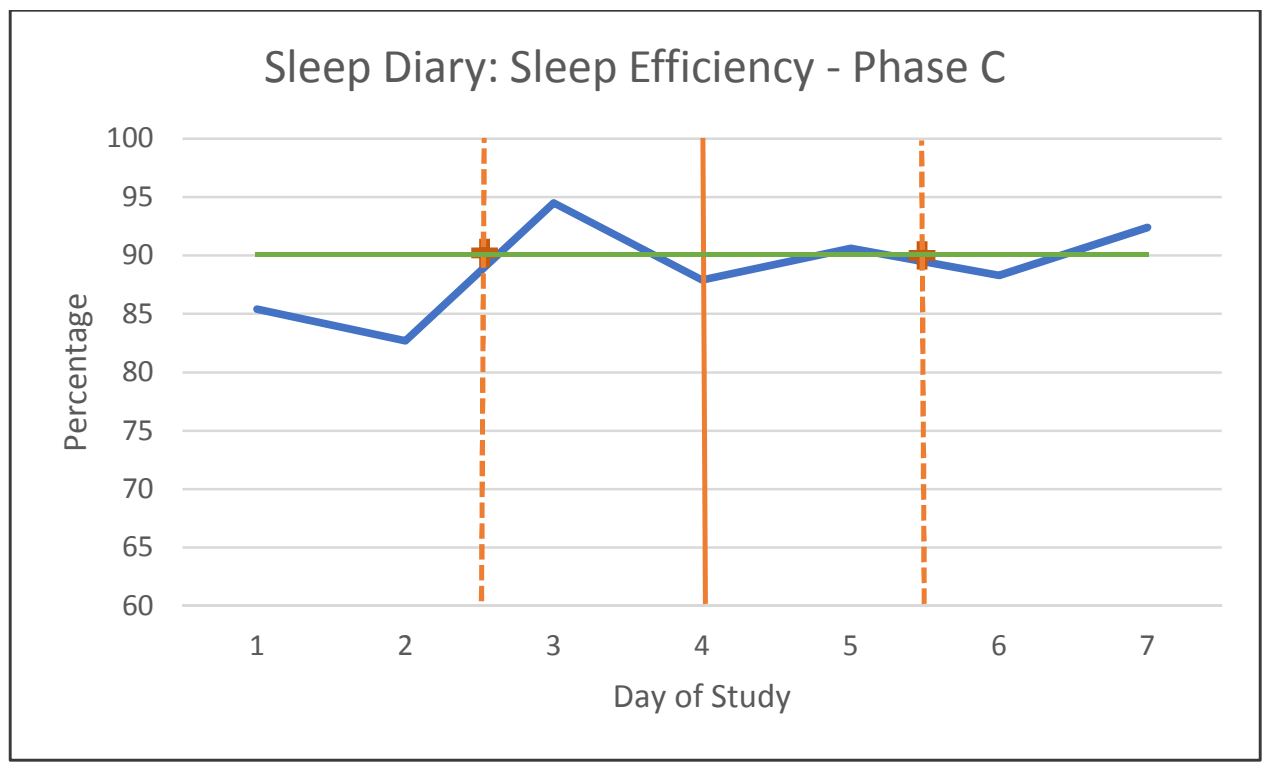

Figure 67. Participant Three Sleep Diary Sleep Efficiency Slope Phase C. The solid orange line represents a dividing line for half of the baseline data. Dashed orange lines represent a dividing line for quarters of the baseline data. The solid green line represents the celeration line.

Variability. The direction of change was variable throughout the intervention phases, indicating inconsistencies in the stability of data.

Inferential statistics. Using the two standard deviation band method, five out of eight data points in phase $\mathrm{D}$, ten out of ten data points in phase $\mathrm{B}$, and seven out of seven data points in phase $\mathrm{C}$ fell outside of the two standard deviation band. This indicated a statistically significant increase in sleep efficiency in all intervention phases using sleep diary data. The C statistic was calculated for the baseline phase, which yielded $\mathrm{C}=0.2596$ with a standard error of 0.3227. The $\mathrm{z}$ score was $0.804(0.804<1.64)$, indicating no significant trend in the baseline data. Baseline data were then combined with data from the intervention phases, yielding $\mathrm{C}=$ 0.8323 with a standard error of 0.171 . The $\mathrm{z}$ score for the combined data was $4.868(4.868>$ 1.64), indicating a statistically significant trend across baseline and intervention phases.

Fitbit Inspire HR. Figure 68 depicts sleep efficiency for all phases of the study using Fitbit data for participant three. 


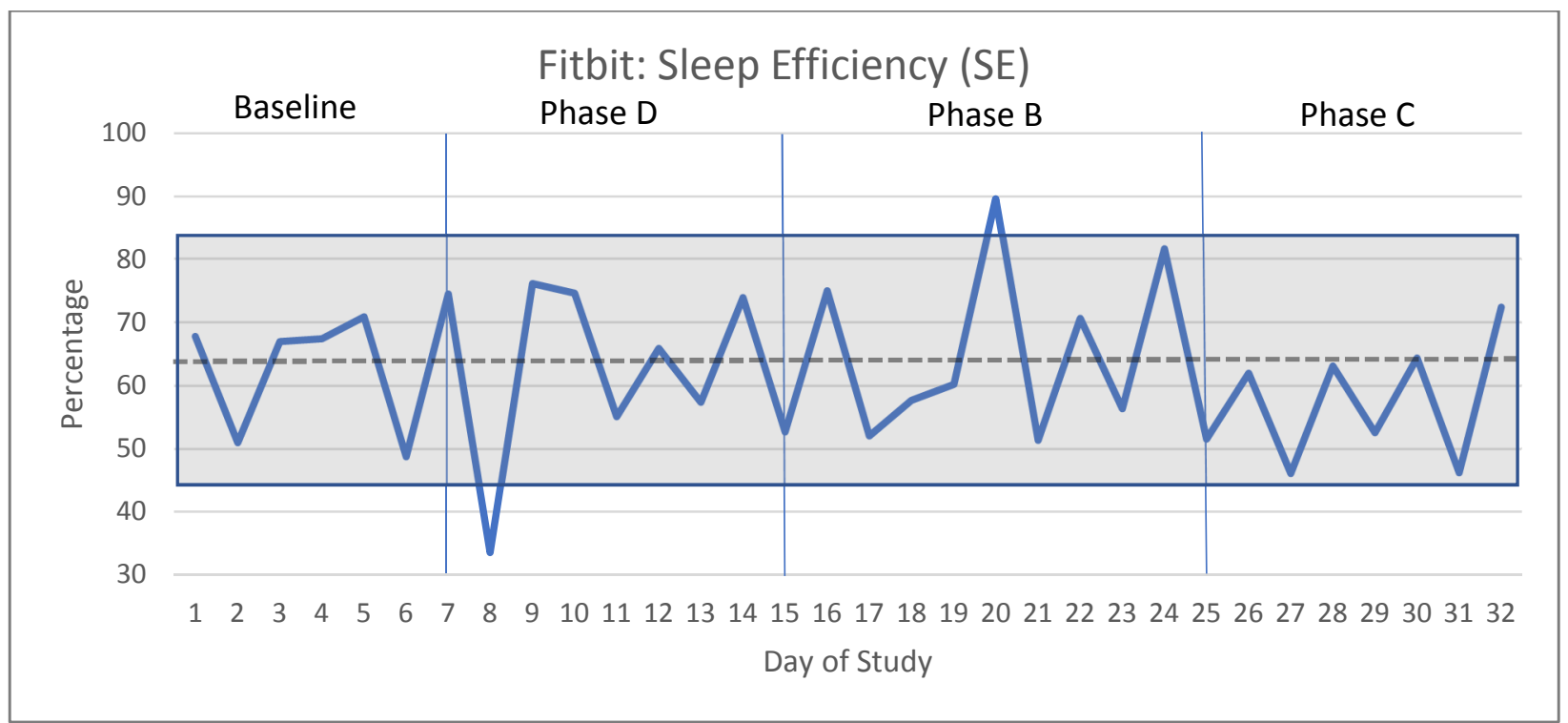

Figure 68. Participant Three Fitbit Sleep Efficiency. Dashed lines represent the mean of the baseline phase. The gray squared box represents the Two Standard Deviation Band.

Using visual analysis techniques of the graph in Figure 68, the level, trend, slope, and variability were calculated for sleep efficiency from the Fitbit data.

Level. The mean value for sleep efficiency of the baseline phase is depicted in Figure 68, which was 63.914 . The mean for phase $\mathrm{D}$ was 61.150 , phase $\mathrm{B}$ was 64.620 , and for phase $\mathrm{C}$ 58.143. This indicated that the average for sleep efficiency increased only for phase B, the second intervention phase.

Trend. Data for sleep efficiency shows no trend for baseline data and intervention phases, indicating fluctuations and no significant changes for sleep efficiency throughout the study.

Variability. The direction of change was variable throughout the phases, indicating unstable data in regards to sleep efficiency. 
Inferential statistics. Using the two standard deviation band method, two data points in separate phases fell outside of the two standard deviation band. This indicated no statistically significant change in sleep efficiency with Fitbit data.

Comparison of sleep diary and Fitbit data. Data were collected simultaneously throughout the study using both the Consensus Sleep Diary and the Fitbit Inspire HR for each of the sleep metrics measured in the study and in each phase of the study. Figure 69 depicts a graphical comparison of these data for sleep efficiency for participant three.

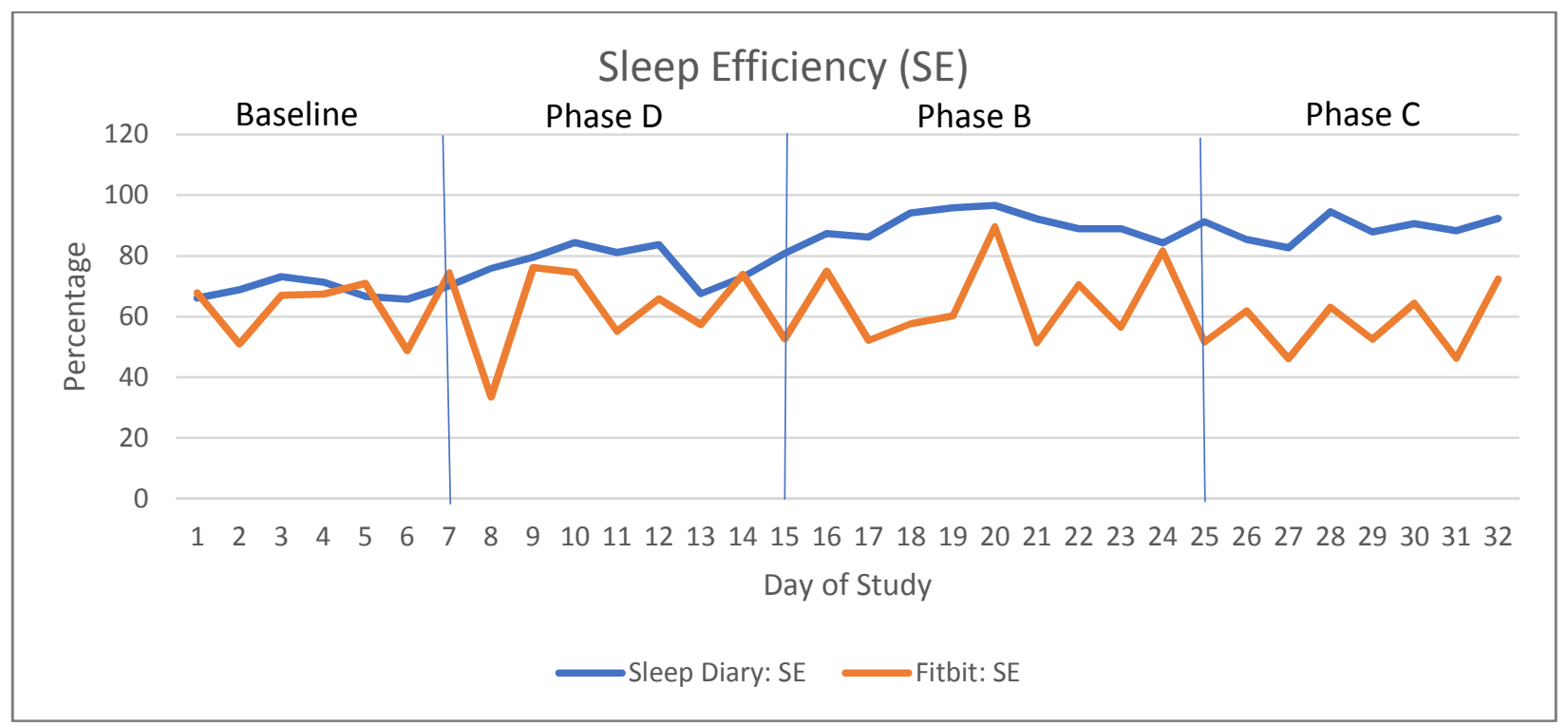

Figure 69. Participant Three Combined Data Sleep Efficiency.

When comparing data for sleep efficiency from the sleep diary and the Fitbit, there was no consistent trend between the two outcome measures. Variability was most similar between sleep diary and Fitbit data in intervention phase B, where sleep efficiency increased for both outcome measures.

\section{Number of Awakenings}

The number of awakenings is graphically depicted in Figure 70 for sleep diary data and in Figure 71 for Fitbit data. 
Consensus Sleep Diary. Figure 70 depicts the number of awakenings for all phases of the study using sleep diary data for participant three.

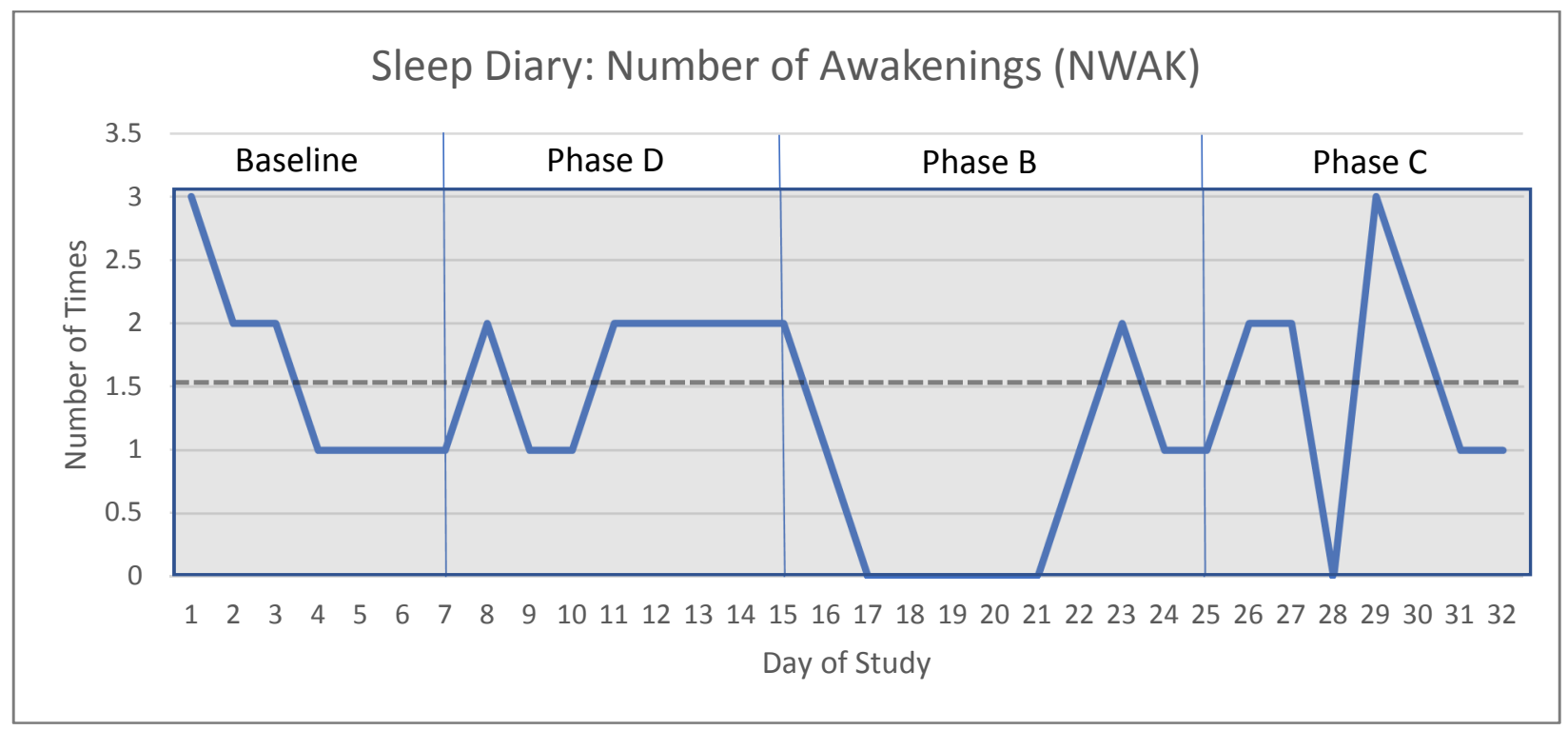

Figure 70. Participant Three Sleep Diary Number of Awakenings. Dashed lines represent the mean of the baseline phase. The gray squared box represents the Two Standard Deviation Band.

Using visual analysis techniques of the graph in Figure 70, the level, trend, slope, and variability were calculated for the number of awakenings from the sleep diary data.

Level. The mean value for number of awakenings of the baseline phase is depicted in Figure 70 , which was 1.57 . The mean for phase D was 1.75 , phase $\mathrm{B}$ was 0.60 , and for phase C 1.57. This indicated that the average number of awakenings only decreased for phase $\mathrm{B}$, the second intervention phase.

Trend. Data for the number of awakenings showed a decelerating trend for baseline data and no trend for intervention phases, indicating fluctuations in the number of awakenings and no significant change throughout the study.

Variability. The direction of change was variable throughout the intervention phases with less variability noted in the baseline phase. 
Inferential statistics. Using the two standard deviation band method, no data points fell outside of the two standard deviation band. This indicated no statistically significant change in the number of awakenings.

Fitbit Inspire HR. Figure 71 depicts the number of awakenings for all phases of the study using Fitbit data for participant three.

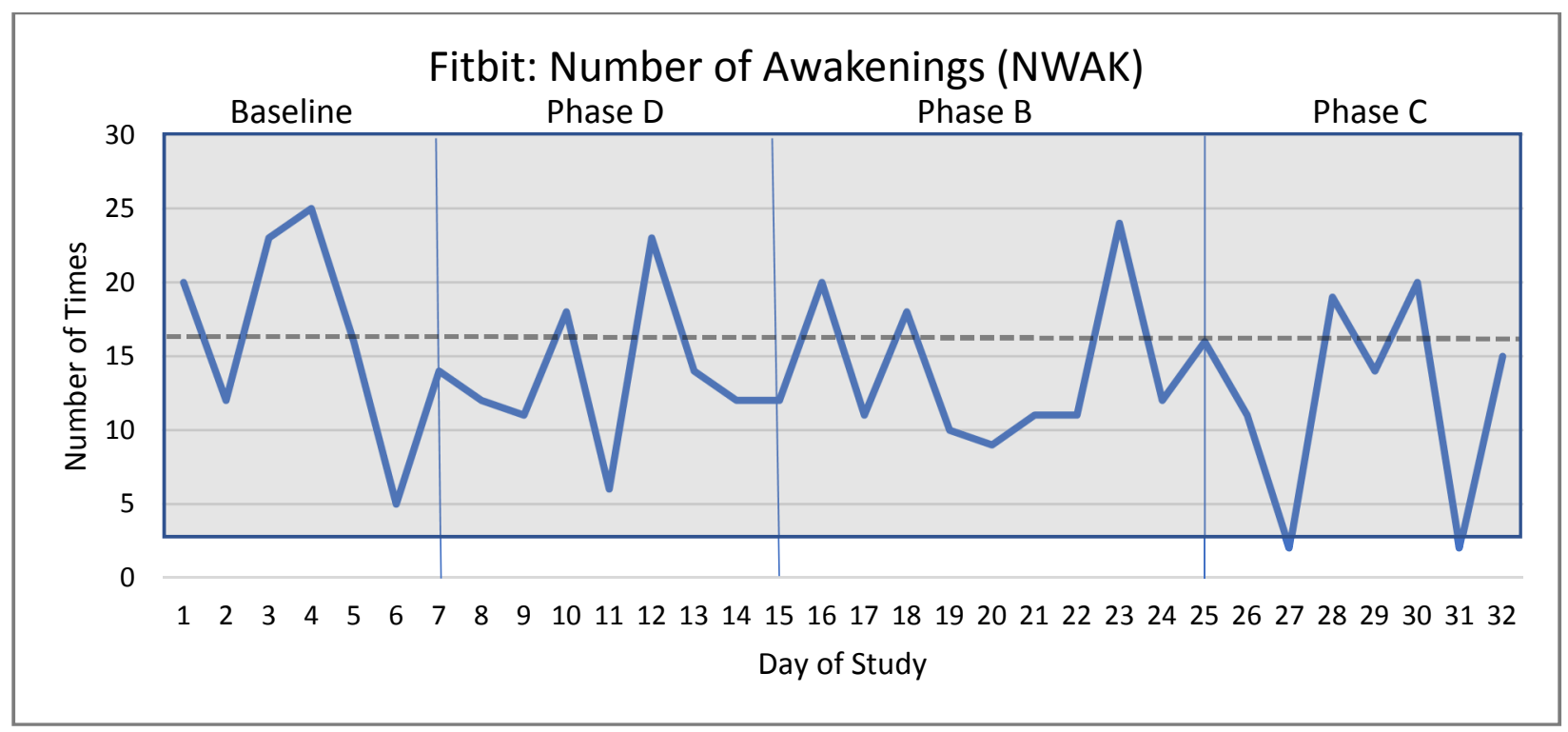

Figure 71. Participant Three Fitbit Number of Awakenings. Dashed lines represent the mean of the baseline phase. The gray squared box represents the Two Standard Deviation Band.

Using visual analysis techniques of the graph in Figure 71, the level, trend, slope, and variability were calculated for the number of awakenings from the Fitbit data.

Level. The mean value for number of awakenings of the baseline phase is depicted in Figure 71 , which was 16.43 . The mean for phase D was 13.50 , phase B was 14.20 , and for phase C 11.86. This indicated that the average number of awakenings decreased for all intervention phases when compared to the baseline phase.

Trend. Data for sleep onset latency showed no trend for baseline data and intervention phases, indicating fluctuations in the number of awakenings. However, the overall trend of data 
showed a mild decelerating trend from baseline to the end of the study, indicating an overall mild decrease in the number of awakenings as the study progressed.

Variability. The direction of change was variable throughout the phases, indicating less stable data.

Inferential statistics. Using the two standard deviation band method, two nonconsecutive data points fell below the two standard deviation band. However, this indicated no statistically significant change in the number of awakenings.

Comparison of sleep diary and Fitbit data. Data were collected simultaneously throughout the study using both the Consensus Sleep Diary and the Fitbit Inspire HR for each of the sleep metrics measured in the study and in each phase of the study. Figure 72 depicts a graphical comparison of these data for the number of awakenings for participant three.

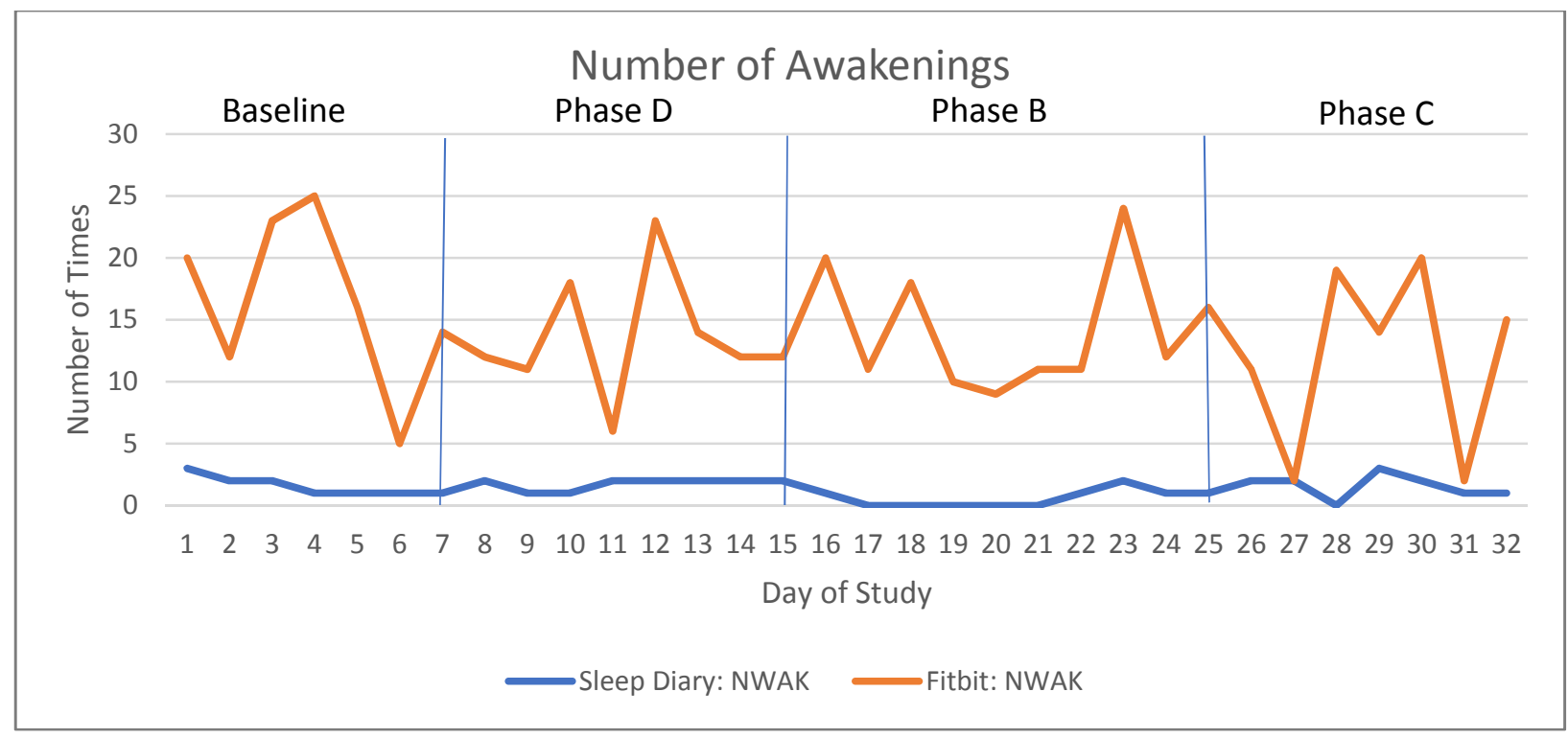

Figure 72. Participant Three Number of Awakenings.

When comparing data for the number of awakenings from the sleep diary and the Fitbit, there was no consistent trend between the two outcome measures. However, the number of awakenings decreased in intervention phase B for both outcome measures. See Table 4 for a 
comparison of the means of each phase for all variables for the sleep diary and the Fitbit device for participant three.

\section{Severity of Insomnia}

A participant's level of insomnia was measured at the beginning of the study, after all interventions were completed, and at a one-month follow-up after interventions were completed. The severity of insomnia was measured using the Insomnia Severity Index, with lower scores indicating less insomnia. Figure 73 shows the results of the Insomnia Severity Index assessments for participant three.

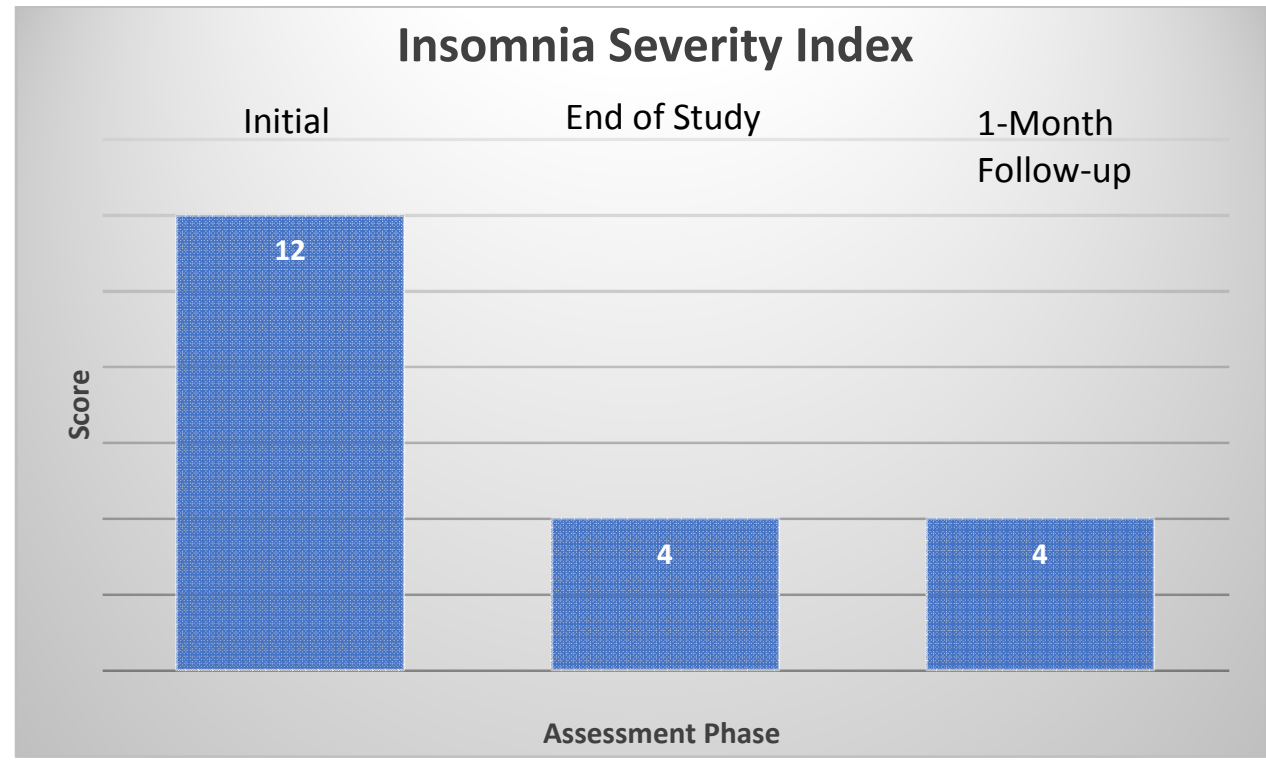

Figure 73. Participant Three Insomnia Severity Index.

Keith's initial Insomnia Severity Index score indicated "sub-threshold," or clinically significant, insomnia (Morin, 1993). The severity of his insomnia decreased after participating in the sleep intervention phases, indicating "no clinical significance" (Morin, 1993). His score remained at this decreased level at the one-month follow-up, indicating a carry-over effect from participating in the sleep interventions. 
Table 4

Participant Three Mean Comparison

\begin{tabular}{|c|c|c|c|c|c|c|c|c|c|c|c|c|c|c|c|c|}
\hline & \multicolumn{3}{|c|}{ Sleep Onset Latency } & \multicolumn{4}{|c|}{ Total Sleep Time } & \multicolumn{4}{|c|}{ Sleep Efficiency } & \multicolumn{5}{|c|}{ Number of Awakenings } \\
\hline & Baseline & Phase D & Phase B & Phase C & Baseline & Phase D & Phase B & Phase C & Baseline & Phase D & Phase B & Phase C & Baseline & Phase D & Phase B & Phase C \\
\hline Diary & 22.86 & 25.00 & 11.00 & 10.71 & 401.43 & 455.00 & 511.00 & 517.14 & 68.871 & 78.263 & 90.610 & 88.829 & 1.57 & 1.75 & 0.60 & 1.57 \\
\hline Fitbit & 10.86 & 24.75 & 38.40 & 31.29 & 371.00 & 356.13 & 357.40 & 340.00 & 63.914 & 61.150 & 64.620 & 58.143 & 16.43 & 13.50 & 14.20 & 11.86 \\
\hline
\end{tabular}




\section{Functional Outcomes of Sleep}

To determine the functional implications of disruptions in sleep, the Functional Outcomes of Sleep Questionnaire was administered at the beginning of the study and after the completion of each intervention phase. Higher scores indicated less disruption in functional outcomes. See Figure 74 for results of the Functional Outcomes of Sleep Questionnaire assessments for participant three.

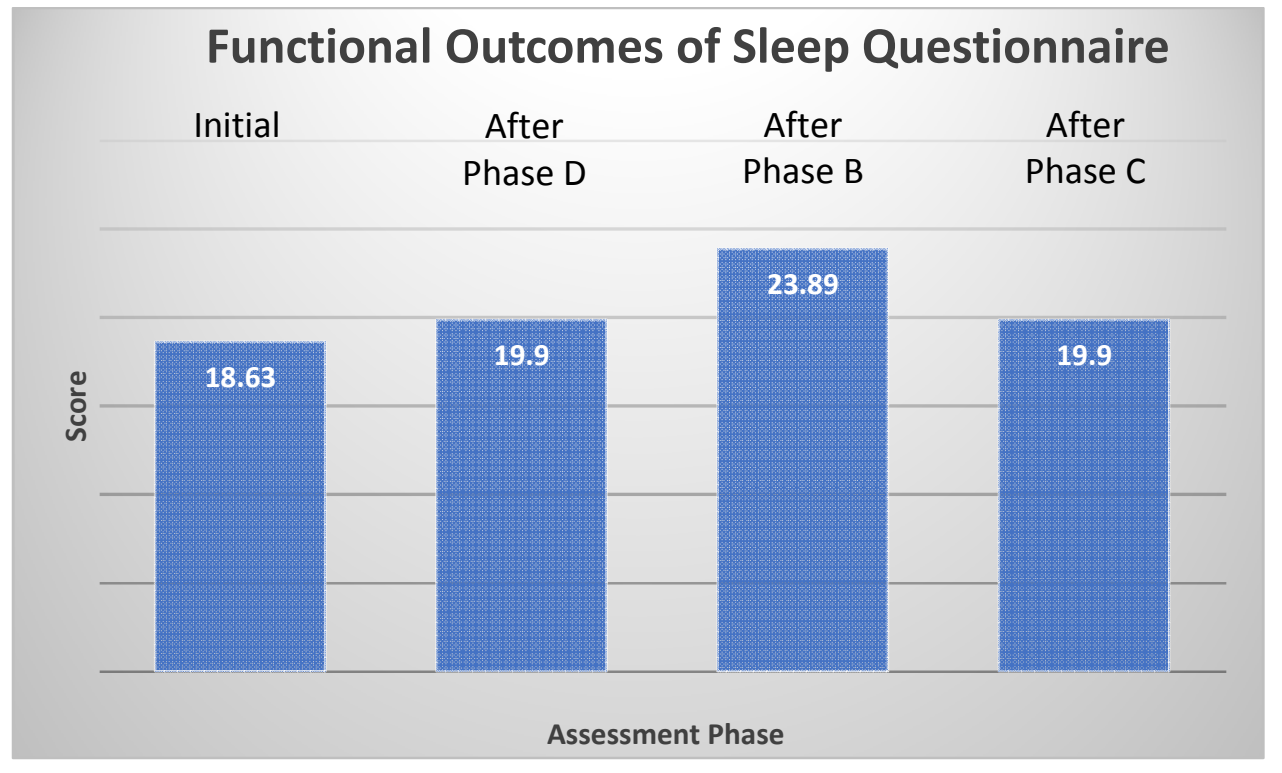

Figure 74. Participant Three Functional Outcomes of Sleep Questionnaire.

The results of Keith's Functional Outcomes of Sleep Questionnaire assessments indicated less disruption in functional outcomes after participating in the intervention phases, with the best functional outcomes noted after participating in intervention phase B.

\section{Sleepiness}

To determine the level of sleepiness of participants while participating in the dissertation study, the Epworth Sleepiness Scale was administered at the beginning of the study and after the completion of each phase of the study. Lower scores indicated less sleepiness. Figure 75 shows the results of the Epworth Sleepiness Scale assessments for participant three. 


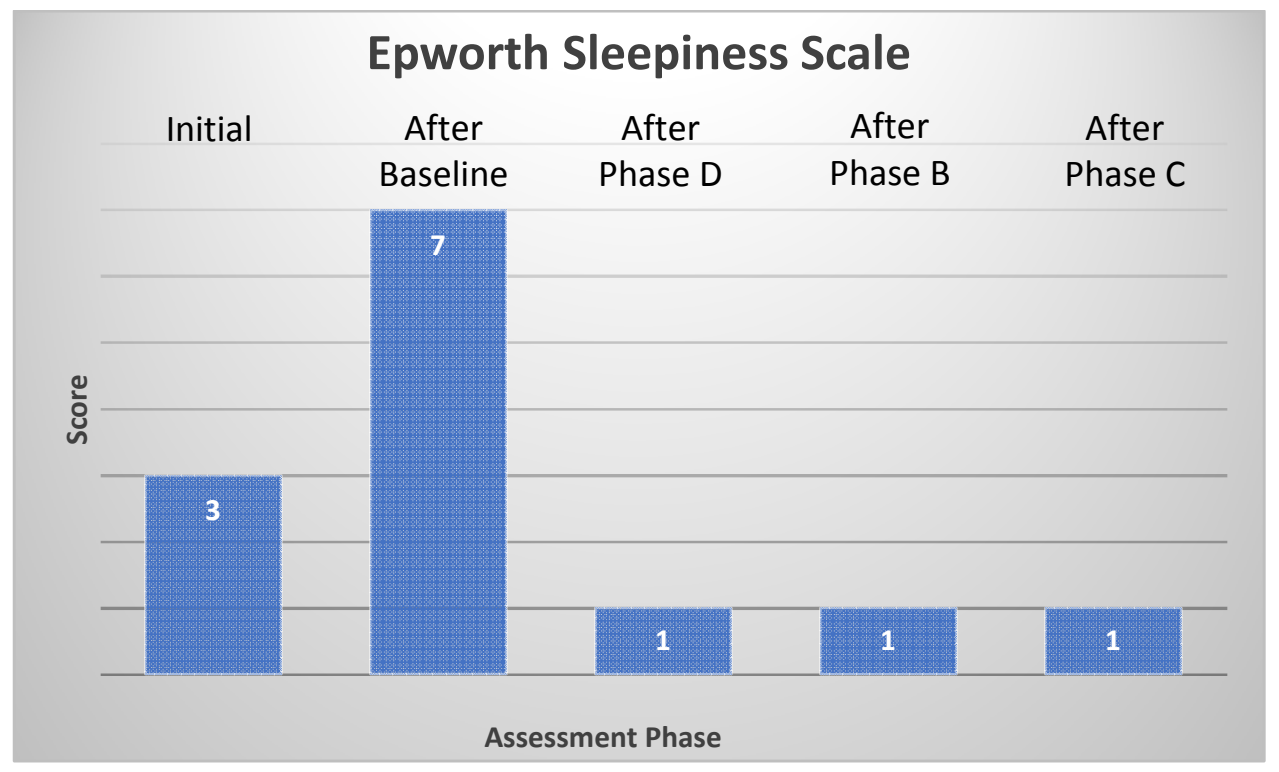

Figure 75. Participant Three Epworth Sleepiness Scale.

The results of Keith's Epworth Sleepiness Scale assessments indicated an increase in sleepiness after the baseline phase and a decrease in sleepiness after participating in the intervention phases, as compared to his initial Epworth Sleepiness Scale score. Keith's initial score and scores after each of the intervention phases indicated "lower normal daytime sleepiness" (Johns, 1991). His score of seven after the baseline phase indicated "higher normal daytime sleepiness" (Johns, 1991) prior to participating in the intervention phases.

In addition to participating in the multicomponent sleep program, participants engaged in semi-structured interviews. Information from the interviews was used to answer the qualitative research questions for the dissertation study. There were two qualitative research questions addressed in the study.

\section{Qualitative Research Questions}

1. What factors affect sleep participation for each participant in the study?

2. What are the occupational implications of decreased sleep after BI? 


\section{Qualitative Outcomes}

To address the qualitative research questions, semi-structured interviews were conducted individually with each research participant using the heuristic process used for phenomenological research outlined by Moustakas (1994). This process involved the initial engagement with participants to discuss the study, where the principal investigator initiated the process of building rapport and trust with participants. Immersion occurred when the principal investigator met with study participants in a community environment that was selected by participants in order to discuss sleep habits, environmental supports or barriers to sleep, changes in sleep participation, and for data collection purposes. Incubation occurred through subsequent meetings and interactions with participants and reflection on participants' statements in order to make meaning of their descriptions and experiences related to disrupted sleep, and illumination occurred when relevant statements from participants evolved into categories and then into themes. By reflecting on data collected through reflexive journaling, potential biases of the principal investigator were identified and eliminated, thus leading to further clarity regarding participants' experiences. This reflexive process served as explication. Finally, culmination of the research in a creative synthesis occurred to depict the meaning and essence of participants' experiences related to disrupted sleep after BI. This qualitative analysis process started with the transcription of participants' recorded interviews with subsequent review of the transcripts.

Each participants' transcripts were repeatedly read, and every participant statement relevant to the topic was reviewed to identify the meaning through the process of horizonalization (Moustakas, 1994). Data were coded by the principal investigator using the NVivo 12 qualitative software system (QSR International, 2018). The principal investigator then convened with an external researcher, who had also independently reviewed and coded 
participant's transcripts, in order to compare codes. The comparison of codes with the external researcher revealed similar codes, suggesting no research bias was imposed on the data. Several codes were identified that related to sleep patterns.

The codes that depicted what sleep was like (i.e., awakenings during the night, factors related to bad sleep, factors related to good sleep, and changes in sleep) were clustered together under the category of sleep quality. Codes that related to factors that were outside of the person (i.e. environmental factors, changes in bedtime routines, and evening routines) were clustered together under the category of external influences on sleep. These two categories related to overall changes in participants' sleep after BI and were linked to the identified theme of changes in sleep patterns. Codes that involved physical factors related to disrupted sleep (i.e. pain, fatigue, and energy) were clustered together under the category of physiological factors, and codes that related to mental or emotional factors affected by disrupted sleep (i.e. increased awareness, unwanted thoughts, and emotional responses) were clustered together under the category of psychological factors. These two categories related to the overall experience of disrupted sleep and were linked to the identified theme of living with sleep disturbance. Once codes were finalized, the four emerging categories were sleep quality, external influences on sleep, physiological factors, and psychological factors. After further review of participants' transcripts, irrelevant expressions were eliminated and common themes of changes in sleep patterns and living with sleep disturbance were identified. These themes and participant's unique experiences guided the individual textural and structural descriptions of the experience (Moustakas, 1994), which are highlighted in the next section. 


\section{Individual Textural and Structural Descriptions of the Experience}

This section presents participant's backgrounds, habits, and routines related to sleep and identifies how sleep affected daily activities for each participant since the BI. The semistructured interview questions and participant responses related to the qualitative research questions. Each participants' name was changed to protect participants' anonymity. Each participants' experiences were related to the final core themes, which are discussed later in this chapter. The first participant interviewed was John.

Participant 1 John. John was a 50-year-old married male who was 28 days post-CVA when the interview was conducted. He lived with his wife and adult son and was independent with basic self-care and mobility. John reported having a long history of sleep deficits, which he indicated were worse after the CVA. He reported that he was planning to see a sleep physician for a possible diagnosis of sleep apnea, which he completed during the third week of study, or the end of the first week of the sleep interventions. He was then diagnosed with sleep apnea and was placed on a CPAP machine at night. John inconsistently wore his CPAP during the study due to reports of discomfort. He was also prescribed two sleep medications, which he took regularly throughout the entire research study as part of his typical bedtime routine.

Bedtime routine. John described his typical bedtime routine as going to bed around 9:00 p.m., using a box fan to keep him cool and for white noise, and regularly putting his phone away at bedtime to reduce distractions. John reported that he typically completed a crossword puzzle and often took a walk with his wife before bedtime but also acknowledged that more exercise could be beneficial for him. He reported that his bed was uncomfortable, stating, "Comfort in bed is a big one for me. I'm not sure if the mattress is too soft." John also reported often getting up in the middle of the night to eat a snack, drink water, and to have an occasional cup of tea for 
relaxation. He indicated that since the CVA, he had increased awareness regarding his sleep habits, stating, "Since the brain injury, I've been actually trying to monitor my sleep and manage my sleep better. I wasn't very good beforehand at managing my sleep.” John discussed several factors he associated with good sleep.

Good sleep. John indicated that good sleep meant getting 8 hours of sleep and taking less than 30 minutes to fall asleep. He also reported that good sleep was getting deep sleep to improve brain functioning, stating, "Getting a good night's sleep... clears the fog” and, "Sleep heals the brain and especially after the stroke." He also indicated that sometimes getting a good night of sleep made him feel more fatigued and that being up for long periods of time sometimes made him more alert. John stated, "Sometimes if I've been up for a long period of time, sometimes I feel like I'm sharp, like I'll be reading books and make connections that I haven't made before." He also mentioned the effects of bad sleep.

Bad sleep. John indicated that a bad night of sleep would not give him deep REM sleep and that he would feel less restful the following day. He also reported that falling sleep was difficult for him, and the experience of dozing off made him nervous. John stated, "That part where you doze off... where you actually just kind of let go... that fly space. I'm kind of scared to go and let go and have that like kind of fly, that free fall." He also reported difficulty staying asleep and having multiple awakenings throughout the night. Even though John reported significant disruptions in sleep, he indicated that he was unsure if poor sleep affected his ability to participate in daily activities the following day. He did mention several factors that affected his sleep.

Influences on sleep. John stated, "Being able to shut my own brain down" was the biggest factor in getting a good night of sleep for him. He also indicated that sleep medication 
and reading a prayer book helped him with getting good sleep. The second participant, Daniel, also shared some similarities in regards to sleep participation.

Participant 2 Daniel. Daniel was a 56-year-old married male who was 55 days postCVA when the interview was conducted. He lived with his wife, adult daughter, and grandchild and was independent with basic self-care and mobility. Daniel indicated having disrupted sleep since the CVA, but also reported a history of chronic sleep apnea with nightly CPAP use that started prior to the CVA. He was regularly taking a prescribed sleep medication throughout the research study as part of his bedtime routine.

Bedtime routine. Daniel described his typical bedtime routine as starting with an evening shower. He then reported briefly watching television with his family and then taking his sleep medication. He reported not eating or drinking after dinner, around 6:00 p.m., to reduce trips to the restroom in the middle of the night. Daniel also reported regularly playing meditation music to fall asleep and going to bed around 9:00 p.m. He reported that his daughter and grandchild went to bed before him and did not disrupt his sleep. He also discussed his idea of good sleep.

Good sleep. Daniel reported that "sleeping 8 hours straight through without getting up" was his idea of a good night of sleep. He also reported a good night would lead to him feeling well rested in the morning. He indicated that falling asleep in 30 minutes or less would be ideal. Good sleep also meant more energy for Daniel. With good sleep, 'I'm better energetic... have energy all day... not tiring out, you know, in the midday. It depends on what kind of activity I have... but, yeah, it'd be a lot of energy during the day." He reported that good sleep would mean being "able to complete all my routines and daily activity without getting tired." He also indicated that a bad night of sleep often had negative effects on him. 
Bad sleep. Daniel reported that a bad night of sleep would limit his productivity the following day. He stated that bad sleep lead to "not being able to complete... the task... I set out to do, getting tired a lot more often..., sitting in my chair falling asleep." He felt that decreased sleep would also negatively affect the healing process. Daniel also reported that falling asleep and going back to sleep after multiple awakenings was very difficult for him, indicating that it took him about an hour to initially fall asleep at night and that he typically woke up about three times per night. He reported feeling increased fatigue the following day after a difficult night of sleep. Daniel also acknowledged significant factors affecting his sleep.

Influences on sleep. Daniel indicated that having a quiet and dark room with meditation music playing helped to improve his sleep. He also indicated that sleeping medication was helpful for him. Daniel reported that shoulder pain from the CVA occasionally interrupted his sleep, stating, "I had a stroke on my left side and this hand it bothers me sometimes, and... it does keep me up every once in a while." He also indicated that disruptive thoughts often made it difficult for him to sleep since the CVA. The third participant, Keith, also presented with disruptions in sleep after a CVA.

Participant 3 Keith. Keith was a 48-year-old married male who was 62 days post-CVA when the interview was conducted. He lived with his wife and two children, and he presented with weakness on his non-dominant side and mild-moderate mobility deficits after the CVA, requiring a wheelchair for the majority of his functional mobility. Keith indicated having disrupted sleep since the CVA, but he was not taking any sleep medications. His wife had to assist him with getting in and out of bed and with his nightly routine prior to bed.

Bedtime routine. Keith described his evening routine as going to the restroom and brushing his teeth before bed. His wife assisted him in the bathroom and with getting into bed. 
Before bedtime, he typically watched television and assisted his sons with their homework. He did not typically consume food or liquids after dinnertime, which occurred around 6:30 or 7:00 p.m. Keith discussed his idea of good sleep.

Good sleep. Keith considered a good night of sleep as "going to bed at a good time, and actually not taking a long time to fall asleep, and just sleep throughout the night without waking up at all until the next morning." He reported that good sleep created less daytime fatigue, allowed him to focus on daily tasks, and made him less distracted by worries, stating:

When I get a good night's sleep I'm more awake, and I'm more aware of what's going on, and I'm not as worried about the things that are going on that I sometimes seem to worry about during the day. Getting a good night's sleep, I don't focus on, I just do what I need to do... without worrying as much as I do.

Bad sleep. Keith reported that bad sleep indicated less energy and increased fatigue the following day, and that daily tasks took longer to complete when he did not have sufficient rest. He stated, 'I'll still get through things, but I can feel it in terms of I've got to slow down and take more time to get things done, but I don't let it stop me from doing what I need to do.” Keith reported difficulty with shutting down his mind and difficulty with falling asleep and staying asleep, reporting that he would wake up at least two or three times in the middle of the night. He also discussed additional factors affecting his sleep.

Influences on sleep. Keith indicated that his environment felt conducive to good sleep, except for his uncomfortable pillow. He indicated that pain in his shoulders would occasionally interrupt his sleep. He also reported that needing extra assistance to get out of bed in the middle of the night to use the restroom affected his sleep, particularly because he had to request help from his wife, which caused psychological distress for him. Psychological distress was common 
amongst all of the research participants and was identified after reviewing significant statements regarding individual textural and structural descriptions of the experiences from research participants as part of the qualitative analysis process. This process also yielded overarching common themes from each of the participants, which were validated through member checks with participants.

\section{Themes of the Findings}

During the qualitative data analysis process, two overarching themes were identified and present for all participants in the research study in relationship to sleep after BI. These themes were living with sleep disturbance and changes in sleep patterns. Subthemes identified under living with sleep disturbance were quieting the mind and emotional regulation, and subthemes under changes in sleep patterns were sleep preparation and sleep participation. The themes and subthemes were used to guide the textural and structural description of the meaning and essence of the experience of disrupted sleep after BI.

\section{Textural and Structural Description of the Meaning and Essence of the Experience}

During the qualitative analysis process, themes were identified for each participant and were then reduced down to overarching common themes amongst all research study participants. Table 5 shows the breakdown of the qualitative analysis process that progressed from the identification of codes, to categories, and then to themes. Identified themes and subthemes are described in more detail in this section. The most outstanding overarching theme among all participants related to living with sleep disturbance after BI.

Living with sleep disturbance. Changes in psychological factors, such as the inability to quiet the mind and challenges with emotional regulation after BI, significantly and regularly affected each participants' sleep. Each participant discussed feelings of worry and emotional 
distress that affected their ability to fall asleep and maintain sleep throughout the night. John discussed concerns over not knowing the cause of his stroke, which often kept him up at night. Table 5

Qualitative Analysis Findings

\begin{tabular}{lll}
\hline Codes & Categories & Themes \\
\hline Awakenings & & \\
Bad Sleep & Sleep Quality & \\
Good Sleep & & Changes in Sleep Patterns \\
Sleep Changes & & \\
\hline & & \\
Environmental Factors & & External Influences on Sleep \\
Bedtime Routine Changes & & \\
Evening Routines & & \\
\hline
\end{tabular}

Pain

Fatigue Physiological Factors

Energy

Living with Sleep Disturbance

Awareness

Unwanted Thoughts Psychological Factors

Emotional Responses

The essence of the subtheme of quieting the mind was captured by John when he said:

I haven't been diagnosed with what happened to cause my stroke, and so that's constantly on my mind. If I get a good night's sleep, it'd basically be able to make it so that I'm not constantly thinking about that.

John also mentioned getting good sleep would mean that, "My brain is basically shut down. I'm not reflecting on things that are coming up or reflecting on things that happened, and I'm actually about to get that deep sleep." He reported reading a praying book at night and praying, "God, please shut my brain down." Daniel also mentioned how worry kept him up at night, stating: 
It can be difficult when I have a lot of thoughts going through my head and, you know, dealing with certain things like, you know, we haven't had a paycheck in a while and paying the bills. Yeah, you just think about those things.

Similarly, Keith discussed how worry affected his sleep, stating:

I do worry about things sometimes more than I should, and that's... a big effect also... if something happens or I know something's going to go on, I start thinking about... that day before I go to bed and sometimes it's still on my mind... when I'm trying to sleep that night.

When asked about the parts of sleep that were difficult for him, Keith reported, "Shutting down my mind to actually fall asleep and also that deep sleep that you want to fall into so that you let everything go and you just rest throughout the night." Keith mentioned that not letting everything bother him made a difference in him being able to get good sleep, but he reported that the emotional distress he felt regarding needing assistance from his wife significantly affected his sleep. Both subthemes under living with sleep disturbance are exemplified when Keith said: I need extra help to get up and go to (the) bathroom and things like that. Those are things that I'm thinking about also. Those are things that are also affecting me in terms of my sleep because I know I don't want to... have to bother someone else to do these things, but I know it's something that I need right now.

Keith also commented on the emotional distress that came with decreased sleep, reporting, "It makes me even more upset because I can’t sleep as much as I want to.” Daniel reported irritability and frustration over increased fatigue and decreased energy when he was not able to get sufficient sleep, and John reported increased anxiety with disruptions in sleep and captured the essence of emotional regulation when he stated: 
I get restless and antsy if I wake up and look at the clock, and I'm hoping that it means I've been asleep for four or five hours, and if it's only like a half hour, I get kind of anxious.

This quote by John also highlighted a common theme of changes in sleep patterns that were experienced by all research study participants.

Changes in sleep patterns. Each of the study participants discussed changes in sleep patterns related to sleep preparation, or the habits and routines related to preparing for sleep (AOTA, 2014), and sleep participation, or sustaining a state of sleep without disruption (AOTA, 2014). Keith depicted the essence of changes in sleep preparation when he recounted: It's changed a little bit because I can't really do anything on my own, so I usually have to wait to get help to go to the bathroom and things like that. Before, I was able to do those things and go to bed.

Daniel also mentioned changes in his routine and changes in sleep participation, stating, "I used to be able to sleep through because I used to go to bed later, but now... have to go to bed earlier. I'm on her schedule." He reported:

We seem to be going to sleep at 9:00, which is earlier. It's an... hour and a half earlier than I normally go to sleep, so I'd say that's what's causing me to wake up in the middle of the night... Getting off the routine would affect it.

Daniel also captured the essence of changes in sleep participation when he recounted, Takes a little while to fall asleep, but I do fall asleep and maybe about an hour or two later, I wake up, go to the bathroom. After that, I come back to bed. Takes me a little while to fall back asleep. Maybe about two hours after that, I have to go to the restroom 
again and... do the same thing over again and come back to bed. It takes me a little while to fall asleep.

Daniel also reported, "I wake up a few more times a night, so it has changed since my injury." He stated, “After I wake up... at night, going back to sleep are difficult. Getting comfortable again in the bed, tossing and turning... It's very difficult to go back to sleep after I wake up."

Each of the participants reported difficulty falling asleep and staying asleep, reporting multiple awakenings throughout the night. Keith indicated that, when he woke up in the middle of the night, he would become upset while focusing on not sleeping, which disrupted his sleep even further. He stated, "It just weighs on me and causes me to continue to focus on not sleeping... which makes me stay awake even more.” This quote exemplified the essence of the experience of both themes of changes in sleep patterns and in living with sleep disturbance in relationship to sleep after BI, which were experienced, in some way, by all research study participants. Examples of these experiences were present in significant statements in the semistructured interviews of each participant and offered a better understanding of the experience of disrupted sleep and the occupational implications of sleep disturbance after BI. The qualitative findings also supported the disruptions in sleep exhibited in the quantitative phase of the study, and the integration of the qualitative and quantitative data is presented in the following section.

\section{Integration of Quantitative and Qualitative Data}

During the mixed methods study, data collected in both phases of the study were integrated in order to have a better understanding of the research problem and to better answer the identified research questions. Data from the quantitative phases showed changes in levels of insomnia, sleepiness, functional outcomes of sleep, sleep onset latency, total sleep time, sleep efficiency, and the number of awakenings experienced by participants at baseline, during 
interventions, and after the intervention phases. Data from the qualitative phase identified factors related to sleep quality, the external influences on sleep, and physiological and psychological factors related to sleep disturbance after BI. The data from both phases were merged to have a better understanding of sleep participation and the experience of living with sleep disturbance after BI.

Baseline sleep deficits were identified when participants reported sleep pattern changes and sleep disturbance after BI in qualitative interviews. This information was corroborated in the quantitative strand with participants' initial Insomnia Severity Index scores and baseline sleep efficiency scores. Participants also discussed the experience of living with sleep disturbance in qualitative interviews. This information was supported by decreased Functional Outcomes of Sleep Questionnaire scores prior to intervention and initial Epworth Sleepiness Scale scores that indicated sleepiness at baseline. Having valuable information from both the qualitative and quantitative strands led to a better understanding how disrupted sleep affects life after BI. These integrated data are graphically depicted in Table 6.

Table 6

\section{Integration of Quantitative and Qualitative Data}

\begin{tabular}{lll}
\hline Quantitative Data & Qualitative Information & Integration \\
\hline $\begin{array}{l}\text { Insomnia Severity Index scores of 12-20 } \\
\text { indicated subthreshold to moderate insomnia \& } \\
\text { baseline sleep efficiency scores ranged from }\end{array}$ & $\begin{array}{l}\text { Identification of sleep pattern } \\
\text { changes and sleep disturbance } \\
\text { af-80 percent for the three participants }\end{array}$ & $\begin{array}{l}\text { Identification of baseline } \\
\text { aleep deficits \& justification } \\
\text { for participation in sleep } \\
\text { program }\end{array}$ \\
$\begin{array}{l}\text { Initial Functional Outcomes of Sleep } \\
\begin{array}{l}\text { Questionnaire scores ranged from 15.79 to } \\
\text { 18.63 out of 20, indicating decreased }\end{array}\end{array}$ & $\begin{array}{l}\text { The reported experience of } \\
\text { functional outcomes prior to intervention \& }\end{array}$ & $\begin{array}{l}\text { Understanding how } \\
\text { disrupted sleep affects life } \\
\text { apter BI }\end{array}$ \\
$\begin{array}{l}\text { Eporth Sleepiness Scale scores of 3-10 } \\
\text { indicated lower normal to mild excessive } \\
\text { daytime sleepiness at baseline for the three } \\
\text { participants }\end{array}$ & & \\
\hline
\end{tabular}




\section{Summary of Results}

In summary, the quantitative data provided information regarding many aspects of sleep that were affected for participants after BI. Data were collected during the sleep program in the quantitative phase to show changes in sleep onset latency, total sleep time, sleep efficiency, and number of awakenings using a sleep diary and a Fitbit device. Sleep assessments were also administered to show changes in insomnia, functional outcomes of sleep disturbance, and sleepiness before, during, and after the interventions. These data showed variable changes for participants throughout the intervention phases and across participants, with some positive, negative, and neutral responses to various interventions noted. Additionally, qualitative data yielded important information about the changes in sleep patterns and living with sleep disturbance after BI. Data were merged to offer a better understanding of sleep disturbance after BI. The next chapter gives a more detailed interpretation of the findings, discusses implications for practice, education, and future research, and identifies limitations of this study. 


\section{Chapter 5: Discussion}

The focus of this chapter is to discuss the findings of the dissertation study and to explore the factors that affect sleep, the occupational implications of disrupted sleep, and the potential benefits of participating in a multicomponent sleep program after BI. Despite the acknowledgment in many scholarly articles and research studies that occupational therapists can and should be addressing sleep deficits with clients (Fung et al., 2013; Gentry \& Loveland, 2013; Leland et al., 2014), there are limited studies documenting the effectiveness of sleep interventions provided by occupational therapists (Gutman et al., 2017; Smallfield \& Lucas Molitor, 2018). Additionally, no previously identified research studies examined both quantitative and qualitative aspects of sleep participation in the same research study.

This dissertation study utilized a mixed methods design in order to better understand the effectiveness of various non-pharmacological sleep interventions and the occupational implications of disrupted sleep for the BI population. The primary quantitative strand of the study utilized multiple sleep-related outcome measures to evaluate many components related to sleep participation during a multicomponent sleep program, and the qualitative strand used semistructured interventions to gain information regarding the meaning and the occupational implications of sleep disturbance. Utilizing a mixed methods study design offered a better understanding of the overall experience of disrupted sleep and information regarding which sleep interventions were most beneficial for this population.

\section{Interpretation of Quantitative Results}

The overall study results regarding the benefits of the specific sleep interventions and the occupational implications of disrupted sleep were somewhat variable for participants and throughout the study. However, many positive outcomes were observed during the study, 
supporting the role of occupational therapy in addressing sleep deficits for participants in the study. Results are discussed in relationship to sleep participation outcomes, the severity of insomnia, functional outcomes of sleep, the level of sleepiness experienced by participants, and the overall benefit of specific sleep interventions.

\section{Sleep Participation Outcomes}

During the quantitative phase of the dissertation study, various outcomes related to sleep were measured for each participant using the Consensus Sleep Diary and the Fitbit Inspire HR. Sleep onset latency, total sleep time, sleep efficiency, and number of awakenings were calculated nightly using both measures.

Sleep onset latency. When reviewing data related to sleep onset latency, outcomes were variable across participants and between the sleep diary and the Fitbit data. However, for two of the three participants, there was an overall decrease in sleep onset latency as the study progressed, based on sleep diary data. This indicated positive changes in relationship to the amount of time that it took for Daniel and Keith to fall asleep after participating in the intervention phases. The order of interventions was variable across participants, and both Daniel and Keith had positive changes in sleep onset latency as the study progressed, indicating a cumulative effect that was independent of the order of interventions. Even though none of the data regarding sleep onset latency showed a statistically significant change, there were some positive changes noted for both Daniel and Keith. Total sleep time data yielded overall positive outcomes for all participants.

Total sleep time. When examining the results related to total sleep time using the sleep diary data, all three participants had an overall increase in total sleep time as the study progressed, indicating positive responses to the interventions that were cumulative with a 
variable order of interventions across participants. John reached statistical significance with an increase in total sleep time for phase $\mathrm{D}$, his last phase of the study, and when comparing the trend from baseline to all intervention phases. Keith reached statistical significance with an increase in total sleep time in phase B, his second sleep intervention phase. When comparing Fitbit data, John had an overall increase in total sleep time with a similar pattern of change between the sleep diary and the Fitbit as the study progressed. However, the other two participants showed no significant trend in total sleep time based on Fitbit data, but did show some similarities in day to day patterns of changes in total sleep time between sleep diary and Fitbit data. Changes in sleep efficiency were overall positive for all participants as well.

Sleep efficiency. When reviewing sleep diary data, all three participants had an overall increase in sleep efficiency as the study progressed, continuing the cumulative effect of improved outcomes that were independent of the order of study interventions. Changes in sleep efficiency for Keith reached statistical significance during all intervention phases and when comparing the trend from baseline to all intervention phases. When reviewing Fitbit data, the overall pattern of change was similar for John when comparing the sleep diary and Fitbit data. Patterns of changes in data between the sleep diary and the Fitbit device were relatively similar for Daniel but were not similar for Keith, who also had the most significant positive change in sleep efficiency based on sleep diary data. Outcomes related to the number of awakenings were variable across participants and between the sleep diary and the Fitbit data.

Number of awakenings. Based on sleep diary data, there was no observable trend in data for John and Keith, but Daniel's number of awakenings did show a cumulative decrease as the study progressed. When reviewing Fitbit data, changes were overall variable for John with an increase in the number of awakenings noted in phases $\mathrm{C}$ and $\mathrm{D}$, which coincides with the 
timing of when he was issued the CPAP device. There was an overall decrease in the number of awakenings for Daniel, and the number of awakenings for Keith was less in all of the intervention phases when compared to baseline data. None of the data regarding the number of awakenings showed a statistically significant change, but there were some positive changes noted for both Daniel and Keith. Daniel and Keith also showed overall positive changes in regards to the severity of insomnia after participating in the study interventions.

\section{Severity of Insomnia}

The severity of insomnia for participants was measured prior to participating in the study, after completing all intervention phases, and at a one-month follow-up. It was measured using the standardized assessment, the Insomnia Severity Index, which is used to measure clinical changes in insomnia over time. Each of the participants needed to score above a seven on the Insomnia Severity Index to be included in the study, indicating clinically significant insomnia. John actually had an increase in his severity of insomnia at the end of the study and was unable to participate in the one-month follow-up due to attrition. However, both Daniel and Keith had a significant decrease in the severity of insomnia after participating in the study, with both indicating no clinically significant insomnia after completing the study. At the one-month follow-up, Daniel had an additional decline in the severity of his insomnia and Keith's score remained consistent with his score after completing the study. For both Daniel and Keith, Insomnia Severity Index scores were low enough that clinical treatment for insomnia was no longer indicated after participating in the sleep interventions. Considering that both participants completed the intervention phases in variable order, there was no order effect for this change. Results were similar in regards to the functional outcomes of sleep. 


\section{Functional Outcomes of Sleep}

Functional and occupational outcomes related to sleep were measured using the Functional Outcomes of Sleep Questionnaire, which assesses the occupational implications of disrupted sleep. The assessment was completed at the beginning of the study and after participating in each of the three intervention phases. John's results were variable, but both Daniel and Keith had an improvement in functional outcomes after each intervention phase when compared to initial assessment scores. This indicated an improvement in occupational engagement for both Daniel and Keith after participating in the sleep interventions. However, there was no discernible order to the level of improvement from specific intervention phases based on the variability of assessment results for all three participants. The level of sleepiness during the study yielded similar outcomes.

\section{Level of Sleepiness}

The level of sleepiness was measured for each participant at the initial meeting and after participating in each phase of the study using the Epworth Sleepiness Scale. John's level of sleepiness increased towards the end of the study, but both Daniel and Keith had significantly lower sleepiness scores after participating in the study interventions when compared to initial sleepiness scores, indicating positive changes for Daniel and Keith. Even though Daniel and Keith had decreased sleepiness throughout the study, there was no discernible order to the level of improvement in sleepiness from specific intervention phases based on the variability of assessment results for all three participants. Despite the variability in quantitative results, several common themes were identified in the qualitative phase of the research study, indicating that each of the participants shared some similarities in regards to their experiences of disrupted sleep after BI. 


\section{Interpretation of Qualitative Findings}

Two overarching themes were identified and present for all participants in the research study in relationship to sleep after BI. Member checks with participants verified that these themes represented the participant experience. The two identified themes were living with sleep disturbance and changes in sleep patterns. Subthemes identified under living with sleep disturbance were quieting the mind and emotional regulation, and subthemes under changes in sleep patterns were sleep preparation and sleep participation. Each participant indicated significant disruptions in sleep patterns, which were also evident in the data collected during the sleep program, and many of these sleep patterns improved after participating in the sleep interventions. Each of the participants also identified challenges with disruptive thoughts and worries that affected their sleep. Participants also indicated that the inability to quiet the mind was also a cause and an effect of struggles with emotional regulation related to living with BI. Identification of these significant findings indicates a need to incorporate sleep interventions aimed at addressing psychological functioning related to sleep disturbance into sleep programs. The psychological aspects of disrupted sleep were not specifically addressed in the dissertation study, possibly limiting the study findings.

\section{Summary of Mixed Methods Study}

In summary, even though outcomes were somewhat variable, the overall results of the study showed positive responses to various non-pharmacological sleep interventions for participants, particularly for both Daniel and Keith. This is likely due to the fact that these two participants had more stable sleep at baseline. Daniel and Keith also had slightly lower initial scores on the Insomnia Severity Index than John. However, John did not achieve baseline stability during the baseline period. He was also diagnosed with obstructive sleep apnea during 
the study and started using a CPAP device, which he inconsistently wore throughout the last two weeks of the study intervention period due to reports of discomfort and dislike of the device. Even though behavioral interventions and sleep hygiene education might still be beneficial for individuals with obstructive sleep apnea (O’Donoghue \& McKay, 2012; Stepnowsky, 2011), these factors likely affected John's ability to achieve better sleep outcomes despite his efforts to participate in the sleep interventions. Another factor that limited the results of the study was the lack of interventions that addressed the psychological aspects of disrupted sleep and participants' ability to quiet the mind.

Each of the study participants identified how continued worry affected their sleep during the qualitative phase of the study, but the study interventions addressed factors related to environmental, behavioral, or occupational modifications to promote improved sleep. Despite alternating the order of interventions for each of the study participants, no order affect was identified related to study outcomes. In fact, most improvements in sleep outcomes were cumulative for participants as the study progressed, indicating that repeated measures over time were most beneficial for participants in regards to improving sleep participation. Evidence of the effectiveness of these interventions is demonstrated by the continued use of various behavioral, environmental, and occupational interventions at the 1-month follow-up with participants. For example, Daniel was still drinking chamomile tea, using white noise, completing afternoon exercise, and showering in the evenings, and Keith was also exercising in the afternoon, keeping the bedroom dark, and regulating bedtimes and waketimes. The sleep outcomes with the most significant improvement across participants were total sleep time and sleep efficiency.

Each participant had improvements in total sleep time and sleep efficiency over the course of the intervention period when reviewing sleep diary data. The potential margin for 
improvement is much larger with total sleep time and sleep efficiency when compared to sleep onset latency and the number of awakenings, which are sleep metrics that are typically measured in much smaller time increments. This could possibly explain some of the increased variability and less significant changes in sleep onset latency and the number of awakenings during the study period. In addition to variability in outcomes related to sleep onset latency and the number of awakenings, there were also inconsistencies between sleep diary and Fitbit outcomes in the dissertation study.

When reviewing outcomes from the sleep diary and the Fitbit Inspire HR, there was much more variability in the Fitbit data. Additionally, the patterns of change in sleep metrics were often dissimilar between the two outcome measures for each participant individually. Several other studies have also identified inconsistencies in sleep outcomes with wearable sleep trackers when compared to other sleep outcome measures (Cook et al., 2017; Kang et al., 2017; Liu et al., 2019; Meltzer et al., 2015), indicating that individuals using Fitbit trackers to evaluate sleep should do so with caution and in conjunction with other sleep outcome measures. However, the overall results and findings from both strands of the study yielded information regarding the negative effects of disrupted sleep, leading to a better understanding of how disrupted sleep affects life after BI. Information obtained in this dissertation study can also be compared to other sleep studies that included multiple sleep outcome measures, various nonpharmacological sleep interventions, or that were designed to understand the implications of sleep disturbance.

\section{Relationship to Literature}

A study conducted by Liu et al. (2019) compared sleep using the Consensus Sleep Diary and the Fitbit Alta, a device similar to the Fitbit Inspire HR utilized in the current study. Even 
though the authors noted that the devices could be used interchangeably for long-term sleep monitoring, they also identified that the sleep diary and the Fitbit device were often inconsistent when measuring sleep and suggested that the variations could be large for some participants (Liu et al., 2019). These results were similar to those identified in the dissertation study, which often showed significant variations between sleep diary and Fitbit data. However, with the noted similarities between the sleep diary and Fitbit data in regards to total sleep time in the dissertation study, there does appears to be some utility with the use of Fitbit devices in regards to sleep tracking, which was also supported by Liu et al. Another study conducted by Lillehei et al. (2015) also utilized a sleep diary and a Fitbit tracker to measure sleep quantity using sleep hygiene and lavender as interventions.

Lillehei et al. (2015) found that, due to technical issues with the Fitbit device, there were levels of missing data, which was also experienced in the dissertation study. When reviewing output data from the Fitbit device in the dissertation study, there were occasional periods of missing data that could not be explained by the participants who also reported wearing the devices throughout the entire night. These data further support the use of a sleep diary in addition to the use of any wearable sleep trackers (Lillehei et al., 2015). The study conducted by Lillehei et al. (2015) utilized only a very short intervention time period, and changes in sleep quantity were not noted between groups. However, results indicated positive changes in sleep quality for participants, which was not measured in the dissertation study. This could be a consideration for future research studies. A study conducted by Ouelett and Morin (2007) utilized a similar study design, and the most significant results were also noted in total sleep time and sleep efficiency, which was similar to the dissertation study. 
Ouelett and Morin's (2007) study also utilized a single-case design, similar to a SSRD, with multiple baselines across participants after BI in an outpatient setting. Using CBT-I, some of the study's interventions included various aspects of sleep hygiene and stimulus control, which was similar to the dissertation study. However, even though the authors suggested the use of objective measures of sleep to support subjective measures in future studies, Ouelett and Morin's outcome measures were all subjective in nature. These outcome measures did include a sleep diary and the Insomnia Severity Index, which were included in the dissertation study. Outcomes of the study yielded positive changes in total sleep time, measured by evaluating total wake time in Ouelett and Morin's study, and sleep efficiency for the majority of participants. Overall positive results from the dissertation study in addition to Ouelett and Morin's study, despite a smaller sample size in the dissertation study, offer further support for several elements of the study design and the continued assessment and intervention for sleep deficits with this population. Another study, conducted by Lu, Krellman, and Dijkers (2016), yielded some positive outcomes with only three participants and after BI, which was the same sample size in the dissertation study.

The study conducted by Lu et al. (2016) utilized CBT-I for individuals after BI and collected data using self-report measures of changes in physiological factors related to sleep. Many of these variables were identified during semi-structured interviews as outcomes of disrupted sleep in the dissertation study, but only sleepiness was measured in the study, thus offering another potential area of investigation in future studies. Like the dissertation study, overall positive trends were noted after participating in sleep interventions, but results were limited due to a small sample size and the limited treatment time with participants. This highlights the significance of prolonged interaction with participants during sleep intervention 
periods but also supports the findings that some positive changes in sleep can occur over a short period of time for individuals after BI when utilizing multicomponent sleep interventions, offering support for other occupational therapists to evaluate and address sleep deficits for clients in a variety of situations and settings even when intervention time periods are limited. One previously conducted occupational therapy-directed sleep study utilized a RCT to compare the effectiveness of three sleep interventions.

Gutman et al. (2017) conducted a 3-week long study using both subjective and objective outcome measures, including a wearable sleep tracker and a sleep diary, to collect data regarding nightly sleep while using a sleep hygiene group as the control in the study. Researchers did not find a significant change in sleep in the control group but did find significant changes in the other intervention groups, supporting positive implications of multicomponent sleep interventions like those utilized in the dissertation study. Similar to the dissertation study, Gutman et al. highlighted the current limitation and the need for future studies documenting the effectiveness of occupational therapy-directed sleep interventions. Fortunately, both studies demonstrated overall positive outcomes that will further support the role of occupational therapy in this growing area of practice and research. Another previously conducted research study yielded similar results as the qualitative strand of the dissertation study.

A qualitative research study conducted by O'Donoghue and McKay (2012) used semistructured interviews to understand the experience of individuals living with a sleep disorder. The overall core theme identified in this study was that the disorder was a "life-changing condition" for participants, which relates the theme of living with sleep disturbance that was identified in the dissertation study. O'Donoghue and McKay also identified psychological wellbeing as a subtheme in their study, which was similar to the subthemes of quieting the mind 
and emotional regulation in the dissertation study, indicating overall similar qualitative findings between the two studies and highlighting the importance of attempting to understand the subjective experience of living with sleep disturbance. In addition to the support of other previously conducted research studies, several guiding theoretical perspectives were utilized in the design of the study and in interpreting the results of the study.

\section{Relationship to Guiding Theoretical Perspectives}

The PEO model was utilized in designing sleep interventions based on person factors, environmental factors, and occupation-based factors that related specifically to the BI population. Interventions based on the dynamic interaction of these factors were utilized to promote occupational performance, or sleep participation, in the dissertation study. Questions during the semi-structured interviews related to the identification of factors that either supported or hindered sleep participation for each participant. Sleep assessments, the sleep diary, and the Fitbit device yielded information related to how changes to personal, environmental, and occupational factors influenced sleep participation for participants. Results of the dissertation study indicated positive outcomes related to a cumulative effect of interventions based on

personal, environmental, and occupation-based factors. These results support the PEO model's premise that the greater the fit between the elements of person, environment, and occupations, the greater the occupational performance (Law et al., 1996), or sleep participation in the dissertation study. OA was also utilized to address factors related to the elements of person, environment, and occupation to promote an adaptive response to sleep interventions in study participants.

The principles of OA were utilized to understand how participants adapted to changes to personal and environmental factors related to sleep to achieve improved sleep participation 
throughout the study. Each participant in the study expressed a desire for mastery when indicating the desire to improve their overall sleep participation. An adaptation gestalt occurred when participants agreed to actively engage in the sleep program, and relative mastery occurred when participants achieved improvements in sleep by following the sleep program in the intervention phases of the study, thus validating the basic premises of OA as a supporting model with the noted positive changes in sleep participation during the dissertation study. Similarly, social-cognitive theory was also utilized to understand how changes to personal, environmental, and occupational factors encouraged participants to make behavior changes related to their sleep.

In initiating the study, personal and environmental factors were evaluated to determine how participants' behaviors either supported or hindered changes related to baseline sleep habits. Participants' behavioral capacity and self-efficacy improved as they participated in regular education, repetitive daily interventions, and repeated outcome measures throughout the study. Improvements in participants' sleep served as positive reinforcement for participants to continue completing the sleep interventions during the dissertation study, and participants made several behavior changes to improve their overall sleep participation throughout the intervention period, thus supporting social-cognitive theory as a guiding theoretical model for this type of research study. The results of the dissertation study offer many broad considerations and implications for practice.

\section{Implications for Practice}

The results and findings of the dissertation study can offer insight and several implications for practice in relationship to when, where, and how to address sleep deficits. Even though this study utilized a SSRD in the quantitative phase, and generalizations cannot be made based on study results, the dissertation study did offer support for occupational therapy to 
successfully evaluate and address sleep deficits for the participants in the study. The study also highlights some broad considerations regarding sleep that can easily be incorporated into occupational therapy practice.

\section{Evaluation of Sleep and Selection of Interventions}

Sleep problems are present for many people and, when compounded by a health condition, sleep deficits can be very detrimental for many occupational therapy clients in regards to overall health, occupational engagement, and quality of life (Leland et al., 2016). This dissertation study supports the valuable role of occupational therapy in assessing and addressing sleep deficits with three clients after BI. However, the sleep assessments, outcome measures, and interventions from this study can be applied to a variety of clients and in a variety of settings with simple modifications, as needed, which is well within occupational therapy's scope of practice and can offer significant benefits to clients.

Sleep is identified as an area of occupation (AOTA, 2014). Therefore, the assessment of sleep and sleep-related factors should be included in occupational therapy evaluations, which is not current practice in most clinical settings. When deficits are identified, occupational therapists might consider adding sleep-related treatment goals using appropriate outcome measures into the occupational therapy plan of care. However, sleep is a unique and subjective experience for each person, and one protocol to address sleep deficits might not be beneficial for every single client and in every single setting. When addressing sleep with clients, occupational therapists can consider which sleep interventions are appropriate for each client based on clientcentered practice.

The sleep program in the dissertation study was designed based on common sleep deficits identified by the BI population, based on previous research and established literature. However, 
in practice, sleep programs can be designed to address the specific needs, wants, and priorities of each client individually. During the dissertation study, additional considerations were given to other individuals and factors that either supported or hindered a participant's sleep, and family members and caregivers were involved in the delivery and evaluation of the effectiveness of the sleep interventions. When practitioners are selecting appropriate sleep interventions for clients, it can be beneficial to have a range of intervention strategies to select from in order to address factors related to personal, environmental, and occupational factors that affect sleep, such as the variety of sleep interventions utilized in the dissertation study. Occupational therapists can use clinical judgment to determine which intervention strategies will be most beneficial for individual clients, also being mindful to not overwhelm clients by implementing too many sleeprelated modifications all at once. Considering that the dissertation study identified no order effect in the delivery of sleep intervention phases, it could be possible to first implement sleep interventions that are the most feasible and then mix and match sleep interventions throughout occupational therapy treatment sessions based on clients' responses to various interventions. However, it is also important to consider that sleep is a complicated process.

Sleep disturbance is variable, subjective to individuals, and can involve many factors. For example, practitioners assessing sleep might consider if a client has difficulty falling asleep, staying asleep, has multiple awakenings throughout the night, and the various factors that might affect sleep for clients. Like those administered during the qualitative phase of the dissertation study, specific sleep-related questions can be asked when evaluating sleep in order to gain a better understanding of a client's subjective experience of sleep and the personal implications of disrupted sleep for each client in the client's own words. The findings from these client interviews can influence the types of sleep assessments administered, how occupational 
therapists collaborate with other healthcare professionals to address sleep, and how sleep is addressed in practice. For example, gaining rich information from clients in regards to sleep might help occupational therapists to determine if sleep will be addressed as an occupation itself or as an occupation that is completed in preparation for participation in other occupations. Finding the right combination of sleep-related interventions for each client can also require close collaboration with clients and caregivers or family members. It might also include ongoing daily tracking of sleep-related outcomes to monitor sleep, to reflect on what is or is not working for clients, and to make adjustments to interventions as needed.

\section{Monitoring Sleep Outcomes}

Another implication for practice involves the outcome measures related to monitoring sleep. As previously discussed, repeated measures of various aspects related to daily sleep participation were used to effectively evaluate and address sleep deficits for the participants in the dissertation study. There are a variety of objective measures of sleep that can be utilized in a variety of clinical settings, including devices such as the Fitbit Inspire HR tracker that was utilized in the dissertation study. However, as identified in the dissertation study, wearable tracking devices are not always consistent or reliable. There were clear discrepancies between the Fitbit device and the sleep diary in this study, suggesting that practitioners and other individuals should exercise caution when utilizing these devices to measure sleep. In order to effectively monitor and evaluate sleep, consistent measures with daily measurement of sleep participation can be utilized throughout the sleep intervention period.

Considering that sleep is unique to each individual and is a subjective experience, subjective measures of sleep, such as a sleep diary, can provide valuable information about daily sleep from the client's perspective. Free printable sleep diaries can be found online, are simple 
and easy to follow, require minimal time to complete, and can provide detailed information about each night of sleep. The Consensus Sleep Diary was a very useful tool in the dissertation study but did require education and training on the use of the tool from the principal investigator. Participants also had occasional difficulty in accurately filling out the diary, requiring additional training with the use of the tool in the early stages of data collection. There are additional sleep diaries available to use with clients, and electronic sleep diaries can also be utilized when appropriate. To monitor progress with sleep-related outcomes, there is also a variety of sleep assessments that can be utilized and administered quickly and with minimal training required, such as those administered in the dissertation study.

When assessing outcomes related to sleep in the dissertation study, consistent sleep assessments were utilized at baseline and at various intervals throughout the intervention process in order to measure progress and to document the response to interventions. There is a large battery of simple sleep assessments that is available for use in practice, and appropriate sleep assessments can be utilized based on the specific treatment goals for clients. For example, the dissertation study measured levels of insomnia using the Insomnia Severity Index, functional outcomes related to sleep using the Functional Outcomes of Sleep Questionnaire, and sleepiness using the Epworth Sleepiness Scale in order to measure responses to sleep interventions. Each of the assessment tools required minimal training and were easily administered to each of the participants. However, it is not always feasible to utilize a wide variety of sleep assessment tools in every practice setting due to various factors such as time restraints. While the Insomnia Severity Index and the Epworth Sleepiness Scale were administered very quickly, the 30-item version of the Functional Outcomes of Sleep Questionnaire required about 15 minutes for administration and considerably longer to score. A shorter version of this assessment tool can be 
administered and scored more quickly if needed, but it assesses fewer functional outcomes. It is important to consider the outcomes being measured when selecting appropriate sleep assessments. For example, the dissertation study measured levels of insomnia, functional outcomes related to sleep, and sleepiness but did not measure sleep quality using a formal sleep assessment.

Having information regarding sleep quality in the dissertation study might have provided a more comprehensive picture of the progress made after participating in the sleep interventions and could be beneficial in order to document progress for many clients. A quick assessment tool that is frequently utilized to measure sleep quality is the PSQI (Mairs \& Mullan, 2015). It was not selected for the dissertation study, primarily to reduce testing fatigue in participants, but the PSQI could be beneficial in practice if the measurement of sleep quality is a desired outcome. Because there are so many sleep assessments available that measure various outcomes related to sleep, practitioners addressing sleep might consider familiarizing themselves with various sleep assessments to determine which assessment will effectively measure progress of sleep-related treatment goals. Ideally, a sleep assessment specific to occupational therapy will be developed and can be utilized in practice in the future. Addressing sleep in practice will also likely require some changes to how occupational therapy services are delivered.

\section{Service Delivery Models}

Occupational therapists and other healthcare practitioners addressing sleep deficits with clients might need to consider the service delivery model for suggesting and implementing changes to factors that affect clients' sleep. In addition to adding items related to the assessment of sleep into every occupational therapy evaluation and adding sleep-related treatment goals to the treatment plan when appropriate, billing models might also need modification to include 
charges related to sleep in order for occupational therapists to be consistently reimbursed for addressing sleep in practice. Occupational therapists might also consider how services are provided and the timing of services related to sleep interventions. For example, despite frequent reminders from the principal investigator and family at home, there were occasional incidents with participants and noncompliance in the dissertation study, suggesting the need for consideration of the delivery of services related to sleep.

A practitioner addressing sleep might consider providing a home visit with clients to evaluate aspects of the sleep environment in person versus relying solely on client reports. He or she might also consider working with clients in the evening hours, which supports improved opportunities for home health practitioners to address sleep and identifies the potential for telehealth interactions with clients in regards to sleep. This would give occupational therapists the opportunity to work with clients on home programs closer to bedtime and to, hopefully, improve the client's compliance with following sleep interventions. Even though addressing sleep in the client's natural environment is ideal, sleep deficits can be addressed early following an acute medical condition, illness, or injury and can continue to be addressed throughout the continuum of care, with many of the suggested sleep interventions being similar to those implemented in the dissertation study.

\section{The Continuum of Care}

The assessment of sleep can be included in all occupational therapy evaluations in every setting, starting with acute care after an acute medical event. Different considerations might need to be made to address sleep for clients depending on the setting, but addressing sleep early on can assist clients in developing healthy sleep habits and can support a quicker recovery in inpatient settings. 
Inpatient settings. Addressing sleep in inpatient settings can be challenging, because many aspects of the environment cannot be easily changed. However, occupational therapists can start addressing sleep even in acute care by working with clients to modify behaviors related to sleep and to reduce nighttime stimuli that causes increased arousal and disrupts sleep (Leland et al., 2014), such as the recommendations provided to participants in the dissertation study. Even in inpatient settings, clients can make simple modifications to their routines, such as wearing eye masks or ear plugs, adjusting temperatures in the room, asking family to bring in pillows or blankets from home for increased comfort, and by participating in relaxation techniques before bed. Clients can also start tracking sleep using sleep diaries, can limit caffeine and use of electronics in evening hours, and can make sure to get out of bed as much as possible during the day to promote nighttime sleep, each of which were also suggested in the dissertation study. Occupational therapists can assist clients with each of these tasks in acute care and in inpatient rehabilitation settings as part of standard occupational therapy practice. Additionally, occupational therapists can play a huge role in collaborating with other health care professionals by creating a platform to discuss sleep with the interdisciplinary team (Tester \& Foss, 2018) in order to promote improved sleep for clients in inpatient settings.

Occupational therapists in inpatient settings can work with nursing staff to minimize nighttime interventions that might disrupt clients' sleep and by educating nursing staff on the importance of sleep and the negative effects of nighttime care on sleep quality and overall health (Tester \& Foss, 2018). Nursing staff can also assist with getting clients out of bed more during the day and with adjusting other factors in the client's room that might inhibit sleep. Occupational therapists can also collaborate with physicians in regards to understanding the connection between various health conditions and sleep, the role of occupational therapy in 
addressing sleep, and in writing appropriate orders for occupational therapy to address sleep in future clients with sleep deficits. Some of these roles for occupational therapy can also translate to outpatient and community settings as well.

Outpatient and community settings. In outpatient and community settings, clients are spending the night in their own homes. Therefore, working with clients regarding sleep in these settings offers care in more naturalistic settings, where sleep interventions can be customized to meet the needs of clients in the actual home environment. An ideal situation would allow occupational therapists to meet with clients in the home or through telehealth meetings in the evening times, closer to the client's actual bedtime, where occupational therapists can at least visualize aspects of the home setting. Working with an interdisciplinary team is still beneficial in these settings, and occupational therapists should still work collaboratively with other health care team members to promote discussion that fosters the understanding of each team member's role in helping to promote healthy sleep habits for clients. An additional implication for practice relates to working with individuals with cognitive impairment. When working with individuals after BI who also have sleep deficits, there can be some additional challenges and considerations to make in order to effectively work with these individuals.

\section{Working with Individuals with Cognitive Impairment}

As identified in the dissertation study, working with individuals with cognitive deficits can pose additional challenges, such as issues with compliance and with accuracy in completing interventions and in documenting responses to outcomes. When cognitive deficits from a BI are compounded by a lack of sleep, these deficits can be heightened and are often very detrimental. Decreased sleep after BI often causes changes in attention, memory, executive functioning, and 
in the learning of verbal and non-verbal information (Duclos et al., 2015), which is also common after BI in general.

Even though the study participants were overall very cooperative, they occasionally required reminders, encouragement, and re-education to follow through with the study protocols and in completing appropriate forms due to memory deficits. During the semi-structured interviews, participants were somewhat withdrawn and often had difficulty with elaborating on responses, despite verbal prompting. These challenges support the need for additional considerations when working with individuals after BI.

As identified by Howrey et al. (2017), many survivors of BI can have lasting cognitive and emotional deficits that can affect the ability to participate in meaningful life activities. Like the individuals who participated in the dissertation study, initiation and forethought can be affected after BI. This lack of initiation in participants possibly affected the outcomes of the dissertation study, and a potential lack of initiation or forethought might have also affected recruitment of additional participants in the study, as potential recruits were not presented with additional information regarding the study if they did not express initial interest with the community re-entry staff once sleep deficits were identified. This implies that those working with individuals after BI might consider variable and alternative ways to assess and gain interest and buy-in with treatments and with clinical programs in order to maximize participation and the potential benefits to clients. Additional considerations should also be made when working with individuals with memory deficits, which are often present after BI and can be worse with decreased sleep.

Each of the study participants presented with decreased memory after BI. Despite frequent reminders from the principal investigator and the participants' spouses, participants still 
had occasional issues with participation in the study due to memory deficits, such as forgetting to complete forms or forgetting to wear or bring the Fitbit device to bi-weekly meetings with the principal investigator. Even though these memory deficits only mildly affected participants' engagement in the dissertation study, it is important to consider that individuals with cognitive deficits will likely require additional supports to actively engage in many activities.

Those with cognitive deficits might need frequent reminders to engage in unfamiliar tasks and to follow instructions. Instructions should also be simple and should be presented in various formats, including written instructions that can be accessed by clients when needed. Like the those involved in the dissertation study, caregivers and family members can be involved in care and can be trained to provide cues and reminders to clients when appropriate and when necessary. Careful consideration should also be made to not overwhelm clients after BI and to allow sufficient time for rest breaks when tasks are cognitively challenging. This was not always possible during the dissertation study due to a lack of time and the rigorous schedules of clients in the community re-entry program, but it is an important consideration to make when working with individuals after BI. The dissertation study also offers many implications for education regarding sleep and the promotion of healthy sleep habits in health care.

\section{Implications for Education}

The dissertation study offers some general implications for education in regards to occupational therapy and other health sciences students, post-professional experiences, interprofessional relationships, and information relayed to occupational therapy clients. To plant the seed of addressing sleep for all occupational therapy clients with sleep deficits, occupational therapy educational programs might consider including education regarding sleep in entry-level occupational therapy programs. 


\section{Entry-Level Education}

The infusion of knowledge regarding the role of occupational therapy in assessing and addressing sleep deficits can start at the university level in entry-level occupational therapy programs. Addressing sleep deficits is still an evolving area in occupational therapy practice and research, and occupational therapy educational programs can include education regarding sleep and wake cycles (Fung et al., 2013) and training in the knowledge and skills required to evaluate and address sleep problems (Tester \& Foss, 2018) to further strengthen this evolving area of occupational therapy practice. This training can include education regarding available assessment tools, a range of appropriate non-pharmacological sleep interventions, how to select appropriate interventions, and how to effectively track sleep outcomes in practice. Training can also include interdisciplinary education using an interprofessional education approach where occupational therapy students can participate in case studies with other health science students to roleplay in appropriate interdisciplinary scenarios that address sleep in various practice settings. With basic education regarding sleep and the role of occupational therapy in addressing sleep deficits, new practitioners could be more likely to address sleep in practice, will have a better understanding of their role and the role of other healthcare professionals in addressing sleep, and might gain interest in pursuing continuing education or additional post-professional training opportunities to improve the ability to effectively manage sleep deficits for clients.

\section{Post-Professional Education}

Occupational therapy practitioners can increase their knowledge base and gain the skills necessary to evaluate and treat sleep deficits by studying sleep and attending professional continuing education courses related to sleep and rehabilitation (Fung et al., 2013). This may require additional education through post-professional training, such as attending workshops led 
by occupational therapists or other health care professionals who address sleep in practice and in research. For example, CBT-I workshops offer occupational therapists basic training in the neuroscience related to sleep, guiding theoretical models for addressing sleep, and in the assessment and delivery of multicomponent sleep interventions. This training could position occupational therapists to be able to work in sleep clinics addressing insomnia and to address sleep deficits in a variety of other clinical settings using non-pharmacological sleep interventions. Other continuing education courses related to sleep could allow occupational therapists to build their knowledge and skills related to sleep and to increase the confidence needed to address sleep deficits for clients in a variety of settings and as part of an interdisciplinary team.

\section{Interdisciplinary Education}

Occupational therapists should not assume that other health care professionals will identify or address sleep deficits with clients (Brown, Wielandt, Wilson, Jones, \& Crick, 2014) and should educate other interdisciplinary team members on the implications of disrupted sleep (Duclos et al., 2015; Tester \& Foss, 2018) and the role of occupational therapists in addressing sleep deficits. Having an interdisciplinary approach to addressing clients' sleep deficits can offer more support for clients and give other healthcare team members a better understanding of their potential roles in addressing and improving sleep for clients as well. Occupational therapists can also educate clients, families, and caregivers about sleep.

\section{Client Education}

Occupational therapists can educate clients about the importance of sleep and the potential physical, mental, psychological, and occupational implications of disrupted sleep. Clients might also be educated on the role of occupational therapy in addressing sleep and how 
simple behavioral, occupational, and environmental modifications can support improved sleep. This education could include the awareness of possible sleep deficits related to specific illnesses or injuries and could be presented to clients in a variety of formats, such as educational videos, handouts, and internet resources. Clients could be educated on variety of sleep tracking systems, such as wearable tracking devices and sleep diaries, and could be educated on additional resources available to address sleep deficits, including the possibility of seeking the expertise of sleep physicians when necessary to further address sleep deficits. Limitations of the dissertation study and recommendations for future study are discussed in the following sections.

\section{Limitations}

There were several limitations in the dissertation study. The nature of sleep, in general, is variable, often making it a challenging area to address in practice and research. This likely contributed to the variable results of the study, especially considering that participants also had a recent brain injury. Individuals after brain injury are not always comprehensive historians, and participants in the dissertation study had memory deficits and difficulty initiating and elaborating on questions regarding sleep habits and other factors related to the study. Participants also needed reminders for accuracy in completion of sleep diary forms and daily intervention checklists. Data were occasionally missing from the forms, requiring the implementation of additional procedures related to missing data. Despite regular meetings and daily reminders to participants, they were not always compliant with completing the interventions, based on responses to sleep diary forms and intervention checklists. The first of the three participants also never achieved baseline stability, was diagnosed with obstructive sleep apnea during the study period and did not regularly wear his CPAP device, and was unable to complete follow-up assessments due to attrition, thus likely limiting his outcomes in the study. Additionally, the 
Fitbit device utilized in the study yielded inconsistent information related to wake or sleep times when compared to each of the participant reports and sleep diary data. In addition to these inconsistencies, the dissertation study focused on the behavioral and environmental factors related to sleep disturbance and failed to address psychological components discussed by each of the study participants in the qualitative phase.

The specific interventions were predetermined prior to initiating the study, and psychological interventions were not included based on the limited amount of time available for participants to be enrolled in the study. These time restrictions related to the limited amount of time that participants were involved in the community re-entry program, in general, which also limited the timeframe for the completion of the study and the length of time for each of the intervention phases. The sample size of the study was also very small, and due to occurrence of a global pandemic during the study period, additional data collection was terminated after only three participants because of an indefinite limited access to the study population and setting. Because this sample was so small and was a convenience sample, the results of the study cannot be generalized to other populations and settings. Additionally, the principal investigator had limited control over several factors related to the study.

Initiating and scheduling initial meetings with research participants and caregivers and regular meetings with participants were contingent on the community re-entry program's rigorous schedule, thus limiting face-to-face interactions and time to gain continued buy-in from participants. All initial interactions with potential participants was coordinated through the secretary of the community re-entry program. Identification of new admits into the community re-entry program and potential research participants was also in the control of the occupational therapists working in the community re-entry program, and the level of collaboration between 
community re-entry staff with the principal investigator was not consistent throughout the study period. There are several recommendations for future research that could address some of these limitations.

\section{Recommendations for Further Study}

In general, having more control in a research study is preferable. To ensure more control over the identification of participants and the arrangement of meetings with potential participants, it is recommended that a researcher complete this type of study in a setting where he or she has a more consistent presence and involvement with staff and potential study recruits. Additionally, meeting with clients in the home setting or using telehealth opportunities would allow for data collection methods in the actual sleep environment, and suggestions and modifications can be made based on the participants' natural environment and observations of their typical habits and routines related to sleep in the home setting. Utilizing a more naturalistic environment with individualized sleep interventions would also likely improve participants' investment in the program and overall compliance with sleep interventions. Considering alternative forms of data collection or a different study design may also be beneficial for future study.

Other methods of data collection for a similar sleep intervention study could include participant observation, caregiver or family interviews or assessments to gain additional information regarding potential barriers to sleep, and participant or caregiver journaling to collect more subjective data regarding participants' feelings and challenges faced during the study period and while participating in the sleep interventions. These methods would offer a deeper understanding of sleep disturbance for a particular population. When working with populations with cognitive deficits, asking open-ended questions with probes or asking for 
specific examples versus simple yes or no questions might provoke more detailed and conversational responses from participants. Initial sleep studies in occupational therapy might also initially include participants with typical cognitive functioning to reduce limitations based on cognitive impairments and then apply findings to those with cognitive deficits in subsequent and more refined research studies. Additionally, considering a sequential research study design where the interventions in the quantitative phase can be customized based on specific sleep problems identified in the initial qualitative phase would allow for more individualistic interventions based on the actual participants in the study.

Future studies might also consider longer intervention periods and including interventions that address the psychological components related to sleep disturbance, such as interventions that include mindfulness or relaxation techniques, and measuring outcomes related to sleep quality versus only measuring sleep quantity. A randomized control trial would also evaluate outcomes of sleep interventions when compared to those in a control group and would include significantly more participants, making the results more generalizable. Incorporating some of these additional recommendations into future studies might further support and expand upon the outcomes identified in this dissertation study by offering more inclusive and richer data that further supports occupational therapy's role in this area of practice and research.

\section{Conclusion}

The dissertation study sought to determine if participating in a multicomponent sleep program for individuals in a community re-entry program after BI improved sleep participation and to determine which sleep interventions, if any, were most beneficial for participants. Additionally, the study sought to determine what factors affected sleep participation and the occupational implications of disrupted sleep for the population. The study results and findings 
are based on the outcomes of three participants who completed semi-structured interviews and a multicomponent sleep program during a mixed method dissertation study. The results were utilized to answer the research questions and to support the role of occupational therapy in evaluating and addressing sleep deficits. Existing literature, scholarly research studies, and guiding theoretical models and frameworks were utilized to design the study and to evaluate study outcomes.

Based on the results of the dissertation study, participating in a multicomponent sleep program was beneficial for study participants in regards to improving various aspects related to sleep quantity, with the most significant improvements noted in total sleep time and sleep efficiency. Two of the three participants also had improvements in relationship to the severity of insomnia, overall sleepiness, and functional outcomes related to sleep. It appeared that each of the sleep interventions were beneficial for participants in a cumulative effect over time, but the results of this study did not identify that any one intervention phase was more beneficial than the others for this population. When determining which factors affected sleep for the population, participants identified various factors related to physiological and psychological functioning, environmental factors, and behaviors and evening routines that either supported or inhibited sleep. Overall, participants indicated being able to complete daily occupations despite disruptions in sleep, but reported concerns regarding energy and fatigue levels when completing daily activities after disrupted sleep. Even though most of the study outcomes did not show statistical significance in changes from baseline to the end of the study, results were overall positive and participants reported satisfaction with participation in the study and their individual study results. Results of this study further support the role of occupational therapy in evaluating and addressing sleep as both an area of occupation and as an activity that prepares individuals for 
engagement in other occupations. Despite the assumption that sleep is a specialized area of practice, the assessment and treatment of sleep parallels every other standard of care in occupational therapy and can be addressed like any other meaningful occupation in occupational therapy education, practice, and research. 


\section{References}

American Occupational Therapy Association. (2008). Occupational therapy practice framework: Domain and process (2nd ed.). American Journal of Occupational Therapy, 62(6), 625683. doi:10.1177/0891988712455237

American Occupational Therapy Association. (2012). Occupational therapy's role in sleep. Retrieved from http://www.aota.org/en/About-Occupational-Therapy/Professionals/HW /Sleep.aspx

American Occupational Therapy Association. (2014). Occupational therapy practice framework: Domain and process (3rd ed.). American Journal of Occupational Therapy, 68(Suppl. 1), S1-S48.

Anke, A., Andelic, N., Skandsen, T., Knoph, R., Ader, T., Manskow, U.,...Røe, C. (2015). Functional recovery and life satisfaction in the first year after severe traumatic brain injury: A prospective multicenter study of a Norwegian national cohort. Journal of Head Trauma Rehabilitation, 30(4), E38-E49. doi:10.1097/HTR.0000000000000080

Arbour, C., Gosselin, N., Levert, M.-J., Gauvin-Lepage, J., Michallet, B., \& Lefebvre, H. (2017). Does age matter? A mixed methods study examining determinants of good recovery and resilience in young and middle-aged adults following moderate-to-severe traumatic brain injury. Journal of Advanced Nursing, 73(12), 3133-3143. doi:10.1111/jan.13376

Bastug, G., Ozel-Kizil, E., Sakarya, A., Altintas, O., Kirici, S., \& Altunoz, U. (2013). Oral trail making task as a discriminative tool for different levels of cognitive impairment and normal aging. Archives of Clinical Neuropsychology, 28(5), 411-417. doi:10.1093/arclin /act035 
Benloucif, S., Orbeta, L., Ortiz, R., Janssen, I., Finkel, S. I., Bleiberg, J., \& Zee, P. C. (2004). Morning or evening activity improves neuropsychological performance and subjective sleep quality in older adults. Sleep, 27(11), 1542-1551.

Berglund, B., Lindvall, T., \& Schewela, D. H. (1999). Guidelines for community noise. Retrieved from http://whqlibdoc/hq/1999/a68672.pdf

Bogdanov, S., Naismith, S., \& Lah, S. (2017). Sleep outcomes following sleep-hygiene-related interventions for individuals with traumatic brain injury: A systematic review. Brain Injury, 31(4), 422-433. doi:10.1080/02699052.2017.1282042

Bootzin, R. R., \& Perlis, M. L. (2011). Stimulus control therapy. In M. Perlis, M. Aloia, \& B. Kuhn (Eds.), Behavioral treatments for sleep disorders: A comprehensive primer of behavioral sleep medicine interventions (pp. 21-30). London, England: Elsevier.

Brain Injury Association of America. (n.d.). Brain injury information. Retrieved from https:// www.biausa.org/brain-injury

Broomsfield, N. M., \& Espie, C. A. (2005). Towards a valid, reliable measure of sleep effort. Journal of Sleep Research, 14(4), 401-407.

Brown, C. A., Wielandt, P., Wilson, D., Jones, A., \& Crick, K. (2014). Healthcare providers' knowledge of disordered sleep, sleep assessment tools and non-pharmacological sleep interventions for persons living with dementia: A national survey. Sleep Disorders, 2014(1), 1-9. doi:10.1155/2014/286274

Burgard, E., Foster, L., Kraus, L., \& Radel, J. (2011). Performing single subject research designs in practice. OT Practice, 22(1), 21-23. 
Buysse, D. J., Reynolds, C. F., Monk, T. H., Berman, S. R., \& Kupfer, D. J. (1989). The Pittsburgh sleep quality index (PSQI): A new instrument for psychiatric research and practice. Psychiatry Research, 28(2), 193-213.

Carney, C. E., Buysse, D. J., Ancoli-Israel, S., Edinger, J. D., Krystal, A. D., Lichstein, K. L., \& Morin, C. M. (2012). The consensus sleep diary: Standardizing prospective sleep selfmonitoring. Sleep, 35(2), 287-302.

Centers for Disease Control and Prevention. (2016). Traumatic brain injury and concussion. Atlanta, GA: Author.

Chenail, R. J. (2009). Interviewing the investigator: Strategies for addressing instrumentation and researcher bias concerns in qualitative research. Qualitative Report, 13(4), 14-21.

Cook, J. D., Eftekari, S. C., Dallmann, E., Sippy, M., \& Plante, D. T. (2018). Ability of the Fitbit Alta HR to quantify and classify sleep in patients with suspected central disorders of hypersomnolence: A comparison against polysomnography. Journal of Sleep Research, 23(1), 1-8. doi:10.1111/jsr.12789

Cook, J. D., Prairie, M. L., \& Plante, D. T. (2017). Utility of Fitbit Flex to evaluate sleep in major depressive disorder: A comparison against polysomnography and wrist-worn actigraphy. Journal of Affective Disorders, 217(2), 299-305.

Cosbey, J., \& Muldoon, D. (2017). EAT-UP'M family-centered feeding intervention to promote food acceptance and decrease challenging behaviors: A single-case experimental design replicated across three families of children with autism spectrum disorder. Journal of Autism and Developmental Disorders, 47(3), 564-578. doi:10.1007/s10803-016-2977-0

Creswell, J. W., \& Plano Clark, V. (2011). Designing and conducting mixed methods research (2nd ed.) Thousand Oaks, CA: Sage. 
Desjardins, J. L. (2016). Analysis of performance on cognitive test measures before, during, and after 6 months of hearing aid use: A single-subject experimental design. American Journal of Audiology, 25(2), 127-141. doi:10.1044/2016_AJA-15-0067

Duclos, C., Beauregard, M., Bottari, C., Ouellet, M., \& Gosselin, N. (2015). The impact of poor sleep on cognition and activities of daily living after traumatic brain injury: A review. Australian Occupational Therapy Journal, 62(1), 2-12. doi:10.1111/1440-1630.12164

Fabiano, R., \& Sharrard, S. (2017). Rehabilitation considerations following mild traumatic brain injury. Journal of Nurse Life Care Planning, 17(1), 26-34.

Fitbit. (2019). Fitbit Inspire and Fitbit Inspire HR fitness trackers. Retrieved from https://www. fitbit.com/shop/inspire

Fung, C., Wiseman-Hakes, C., Stergiou-Kita, M., Nguyen, M., \& Colantonio, A. (2013). Time to wake up: Bridging the gap between theory and practice for sleep in occupational therapy. British Journal of Occupational Therapy, 76(4), 384-386.

Gentry, T. \& Loveland, J. (2013). Sleep: Essential to living life to its fullest. OT Practice, 24(1), 9-14.

Green, A. (2008). Sleep, occupation and the passage of time. British Journal of Occupational Therapy, 71(8), 339-347.

Green, A. (2015). Sleep and occupation. In A. Green \& C. Brown (Eds.), An occupational therapist's guide to sleep and sleep problems (pp. 22-32). Philadelphia, PA: Kingsley.

Green, A., \& Durant, C. (2015). Time for bed: Historical and cultural factors, and time use. In A. Green \& C. Brown (Eds.), An occupational therapist's guide to sleep and sleep problems (pp. 56-76). Philadelphia, PA: Kingsley. 
Green, A., \& Hicks, J. (2015). Assessment and non-pharmacological management of insufficient and excessive sleep. In A. Green \& C. Brown (Eds.), An occupational therapist's guide to sleep and sleep problems (pp. 131-159). Philadelphia, PA: Kingsley.

Green, A., \& Nakopoulou, E. (2015). A place to sleep. In A. Green \& C. Brown (Eds.), An occupational therapist's guide to sleep and sleep problems (pp. 327-342). Philadelphia, PA: Kingsley.

Green, A., \& Wilson, S. (2015). The reason of sleep. In A. Green \& C. Brown (Eds.), An occupational therapist's guide to sleep and sleep problems (pp. 33-55). Philadelphia, PA: Kingsley.

Gutman, S. A., Gregory, K. A., Sadlier-Brown, M. M., Schlissel, M. A., Schubert, A. M., Westover, L. A., \& Miller, R. C. (2017). Comparative effectiveness of three occupational therapy sleep interventions. Occupational Therapy Journal of Research, 37(1), 5-13. doi:10.1177/1539449216673045

Howrey, B. T., Graham, J. E., Pappadis, M. R., Granger, C. V., \& Ottenbacher, K. J. (2017). Trajectories of functional change after inpatient rehabilitation for traumatic brain injury. Archives of Physical Medicine and Rehabilitation, 98(8), 1606-1613. doi:10.1016/j.apmr .2017 .03 .009

Jackson, J. P, \& Schkade, J. K. (2001). Occupational adaptation model versus biomechanicalrehabilitation model in the treatment of patients with hip fractures. American Journal of Occupational Therapy, 55(5), 531-537.

Johns Hopkins University. (2016). SBCC implementation kits in emergencies. Retrieved from https://sbccimplementationkits.org/sbcc-in-emergencies/social-cognitive-learning-theory/ 
Johns, M. W. (1991). A new method for measuring daytime sleepiness: The Epworth sleepiness scale. Sleep, 14(6), 540-545.

Kang, S-G., Kang, J. M., Ko, K-P., Park, S-C., Mariani, S., \& Weng, J. (2017). Validity of a commercial wearable sleep tracker in adult insomnia disorder patients and good sleepers. Journal of Psychosomatic Research, 97(1), 38-44.

Kortte, K. B., Horner, M. D., \& Windham, W. K. (2002). The trail making test, part B: Cognitive flexibility or ability to maintain set? Applied Neuropsychology, 9(2), 106-109. doi:10 $.1207 / \mathrm{S} 15324826 \mathrm{AN} 0902$

Kosecki, D. (2017, March 6). Your heart rate is the key to smarter sleep stages: Here's why [Web log post]. Retrieved from https://blog.fitbit.com/heart-rate-during-sleep-stages/

Kyung-Lim, J., Won-Hyoung, K., Ha-Yoon, C., Chan-Hyuk, P., Eun-Suk, K., Sook-Joung, L.,...Han-Young, J. (2017). The effect of sleep disturbances on the functional recovery of rehabilitation inpatients following mild and moderate stroke. American Journal of Physical Medicine and Rehabilitation, 96(10), 734-740. doi:10.1097/PHM.0000.0000 .00000744

Law, M., Cooper, B., Strong, S., Stewart, D., Rigby, P., \& Letts, L. (1996). The personenvironment-occupation model: A transactive approach to occupational performance. Canadian Journal of Occupational Therapy, 46(9), 829-837.

Leland, N. E., Fogelberg, D., Sleight, A., Mallinson, T., Vigen, C., Blanchard, J.,...Clark, F. (2016). Napping and nighttime sleep: Findings from an occupation-based intervention. American Journal of Occupational Therapy, 70(4), 1-7. doi:10.5014/ajot.2016.01765

Leland, N. E., Marcione, N., Schepens Niemiec, L. S., Kelkar, K., \& Fogelberg, D. (2014). What is occupational therapy's role in addressing sleep problems among older adults? 
Occupational Therapy Journal of Research, 34(3), 141-149. doi:10.3928/15394492 $.20140513-01$

Lenz, A. S., \& Lancaster, C. (2017). A mixed-methods evaluation of intensive trauma-focused programming. Journal of Counseling and Development, 95(1), 24-34. doi:10.1002/jcad .12114

Lillehei, A. S., Halcon, L. L., Savik, K., \& Reis, R. (2015). Effect of inhaled lavender and sleep hygiene on self-reported sleep issues: A randomized control trial Journal of Alternative and Complementary Medicine, 21(7), 430-438. doi:10.1089/acm.2014.0327

Liu, J., Wong, W. T., Zwetsloot, I. M., Hsu, Y. C., \& Tsui, K. L. (2019). Preliminary agreement on tracking sleep between a wrist-worn device Fitbit Alta and Consensus Sleep Diary. Telemedicine Journal and E-Health, 30(1), 11-20.

Liu, W., Ploderer, B., \& Hoang, T. (2015, December). In bed with technology: Challenges and opportunities for sleep tracking. Paper presented at the Proceedings of the annual meeting of the Australian Special Interest Group for Computer Human Interaction, Parkville, Australia.

Lobo, M. A., Moeyaert, M., Baraldi Cunha, A., \& Babik, I. (2017). Single-case design, analysis, and quality assessment for intervention research. Journal of Neurologic Physical Therapy, 41(3), 187-197. doi:10.1097/NPT.0000000000000187

Lorenz, R. A., Budhathoki, C. B., Kalra, G. K., \& Richards, K. C. (2014). The relationship between sleep and physical function in community-dwelling adults. Family and Community Health, 37(4), 298-306. 
Lu, W., Krellman, J. W., \& Dijkers, M. P. (2016). Can cognitive behavioral therapy for insomnia also treat fatigue, pain, and mood symptoms in individuals with traumatic brain injury? A multiple case report. NeuroRehabilitation, 38(1), 59-69. doi:10.3233/NRE-151296

Mairs, L., \& Mullan, B. (2015). Self-monitoring vs. implementation intentions: A comparison of behaviour change techniques to improve sleep hygiene and sleep outcomes in students. International Journal of Behavioral Medicine, 22(6), 635-644. doi:10.1007/s12529-015 .9467-1

Martin, J. L., Marler, M. R., Harker, J. O., Josephson, K. R., \& Alessi, C. A. (2007). A multicomponent nonpharmacological intervention improves activity rhythms among nursing home residents with disrupted sleep/wake patterns. Journals of Gerontology Series A: Biological Sciences and Medical Sciences, 62(1), 67-72.

Mastin, D. F., Bryson, J., \& Corwyn, R. (2006). Assessment of sleep hygiene using the sleep hygiene index. Journal of Behavioral Medicine, 29(3), 223-227.

Mathias, J. L., \& Alvaro, P. K. (2012). Prevalence of sleep disturbances, disorders, and problems following traumatic brain injury: A meta-analysis. Sleep Medicine, 13(7), 898-905. doi:10.1016/j.sleep.2012.04.006

Meltzer, L. J., Hiruma, L. S., Avis, K., Montgomery-Downs, H., \& Valentin, J. (2015). Comparison of a commercial accelerometer with polysomnography and actigraphy in children and adolescents. Sleep, 38(8), 1323-1330. doi:10.5665/sleep.4918

Meyer, A. (1922). The philosophy of occupational therapy. Archives of Occupational Therapy, $1(1), 1-10$. 
Micklewright, J. L., Yutsis, M., Smigielski, J. S., Brown, A. W., \& Bergquist, T. F. (2011). Point of entry and functional outcomes after comprehensive day treatment participation. Archives of Physical Medicine and Rehabilitation, 92(12), 1974-1978.

Monk, T. H., Reynolds, C. F., Kupfer, D. J., Buysse, D. J., Coble, P. A. , Hayes, A. J.,...Ritenour, A. M. (1994). The Pittsburg sleep diary. Journal of Sleep Research, 3(2), 111-120.

Morhardt, D. R., Luckenbaugh, A., Goldstein, C., \& Faerber, G. J. (2017). Determining resident sleep during and after call with commercial sleep monitoring devices. Urology, 106(1), $39-44$.

Morin, C. M. (1993). Insomnia: Psychological assessment and management. New York, NY: Guilford Press.

Moscou-Jackson, G., Allen, J., Smith, M. T., \& Haywood, J. C. (2016). Psychometric validation of the Insomnia severity index in adults with sickle cell disease. Journal of Health Care for the Poor and Underserved, 27(1), 209-218.

Moustakas, C. (1994). Phenomenological research methods. Thousand Oaks, CA: Sage.

Nakopoulou, E., Gaylarde, K., \& Green, A. (2015). Sleep disturbance in neurological conditions. In A. Green \& C. Brown (Eds.), An occupational therapist's guide to sleep and sleep problems (pp. 281-290). Philadelphia, PA: Kingsley.

National Cancer Institute. (2005). Theory at a glance: A guide for health promotion practice. (2nd ed.). Bethesda, MD: National Institutes of Health.

National Sleep Foundation. (n.d.). The sleep disorders. Retrieved from http://sleepdisorders .sleepfoundation.org/

Neuendorf, R., Wahbeh, H., Chamine, I., Yu, J., Hutchison, K., \& Oken, B. S. (2015). The effects of mind-body interventions on sleep quality: A systematic review. Evidence- 
Based Complementary and Alternative Medicine, 2015(1), 1-17. doi:10.1155/2015 1902708

Nørgaard, M. W., Pedersen, P. U., \& Bjerrum, M. (2018). Understanding how patients use visualization during ablation of atrial fibrillation in reducing their experience of pain, anxiety, consumption of pain medication and procedure length: Integrating quantitative and qualitative results. Applied Nursing Research, 39(2), 229-240. doi:10.1016/j.apnr .2017 .11 .026

O’Donoghue, N., \& McKay, E. A. (2012). Exploring the impact of sleep apnoea on daily life and occupational engagement. British Journal of Occupational Therapy, 75(11), 509-516.

Ottenbacher, K. J. (1986). Evaluating clinical change: Strategies for occupational and physical therapists. Baltimore, MD: Williams \& Wilkins.

Ouellet, M. C., \& Morin, C. M. (2004). Cognitive behavioral therapy for insomnia associated with traumatic brain injury: A single-case study. Archives of Physical Medicine and Rehabilitation, 85(8), 1298-1302.

Ouellet, M. C., \& Morin, C. M. (2007). Efficacy of cognitive-behavioral therapy for insomnia associated with traumatic brain injury: A single-case experimental design. Archives of Physical Medicine and Rehabilitation, 88(12), 1581-1592.

Portney, L. G., \& Watkins, M. P. (2015). Foundations of clinical research: Applications to practice (3rd ed.). Philadelphia, PA: Davis.

Posner, D., \& Gehrman, P. R. (2011). Sleep hygiene. In M. Perlis, M. Aloia, \& B. Kuhn (Eds.), Behavioral treatments for sleep disorders: A comprehensive primer of behavioral sleep medicine interventions (pp. 31-43). London, England: Elsevier.

QSR International. (2018). NVivo 12. Melbourne, Australia: Author. 
Raphaelson, M., \& Inati, S. K. (2012). Treating sleep disorders in neurology practice. Neurologic Clinics, 30(4), 1007-1025. doi:10.1016/j.ncl.2012.08.015

Richardson, G. S. (2007). Human physiological models of insomnia. Sleep Medicine, 8(1), 9-14.

Ryu, M. J., Park, J. S., \& Park, H. (2012). Effect of sleep-inducing music on sleep in persons with percutaneous transluminal coronary angiography in the cardiac care unit. Journal of Clinical Nursing, 21(7), 728-735. doi:10.1111/j.1365-2702.2011.03876.x

Sasaki, Y. (2012). Recent progress on neuroimaging studies on sleeping brain. Brain Nerve, 64(6), 611-619.

Schkade, J. K., \& McClung, M. (2001). Occupational adaptation in practice: Concepts and cases. Thorofare, NJ: SLACK.

Schkade, J. K., \& Schultz, S. (1992). Occupational adaptation: Toward a holistic approach for contemporary practice, part 1. American Journal of Occupational Therapy, 46(9), 829837.

Shepherd Center. (2018). Brain injury rehabilitation and recovery. Retrieved from https://www .shepherd.org/patient-programs/brain-injury

Smallfield, S., \& Lucas Molitor, W. (2018). Occupational therapy interventions addressing sleep for community-dwelling older adults: A systematic review. American Journal of Occupational Therapy, 72(4), 1-9. doi:10.5014/ajot.2018.031211

Smith, M. T., \& Wegener, S. T. (2003). Measures of sleep: The insomnia severity index, medical outcomes study (MOS) sleep scale, Pittsburg sleep diary (PSD), and Pittsburg sleep quality index (PSQI). Arthritis Care and Research, 49(5S), S184-S196. doi:10.1002/art .11409 
Spira, A. P., Covingsky, K., Rebok, G. W., Punjabi, N. M., Stone, K. L., Hillier, T. A.,...Yaffe, K. (2012). Poor sleep quality and functional decline in older women. Journal of American Geriatrics Society, 60(6), 1092-1098. doi:10.1111/j.1532.5415.2012.03968.x

Stepanski, E. J., \& Wyatt, J. K. (2003). Use of sleep hygiene in the treatment of insomnia. Sleep Medicine Reviews, 7(3), 215-225.

Stepnowsky, C. (2011). Sleep apnea self-management program. In M. Perlis, M. Aloia, \& B. Kuhn (Eds.), Behavioral treatments for sleep disorders: A comprehensive primer of behavioral sleep medicine interventions (pp. 195-210). London, England: Elsevier.

Straus, E., Sherman, E. M. S., \& Spreen, O. (2006). A compendium of neuropsychological tests: Administration, norms, and commentary. New York, NY: Oxford University Press.

Suh, M., Choi-Kwon, S., \& Kim, J. S. (2014). Sleep disturbances after cerebral infarction: Role of depression and fatigue. Journal of Stroke and Cerebrovascular Diseases, 23(7), 19491955. doi:10.1016/j.jstrokecerebrovasdis.2014.01.029

Teddlie, C., \& Tashakkori, A. (2009). Paradigm issues in mixed methods research. In C. Teddlie \& A. Tashakkori (Eds.), Foundations of mixed methods research: Integrating quantitative and qualitative approaches in the social and behavioral sciences (pp. 83105). Thousand Oaks, CA: Sage.

Tester, N. J., \& Foss, J. J. (2018). The issue is - Sleep as an occupational need. American Journal of Occupational Therapy, 72(11), 7201347010p1-7201347010p4. doi:10.5014/ajot.2018 .020651

Tse, L., \& Hall, W. (2008). A qualitative study of parents' perceptions of a behavioural sleep intervention. Child, 34(2), 162-172.

U.S. Department of Health and Human Services. (2018). Stroke. Washington, DC: Author. 
Van Bogaert, P., Peremans, L., Van Heusden, D., Verspuy, M., Kureckova, V., Van de Cruys, Z., \& Franck, E. (2017). Predictors of burnout, work engagement and nurse reported job outcomes and quality of care: A mixed method study. BMC Nursing, 18(1), 1-14. doi:10 $.1186 / \mathrm{s} 12912-016-0200-4$

Vitiello, M. V., Rybarczyk, B., Von Korff, M., \& Stepanski, E. J. (2009). Cognitive behavioral therapy for insomnia improves sleep and decreases pain in older adults with co-morbid insomnia and osteoarthritis. Journal of Clinical Sleep Medicine, 5(3), 355-362.

Wagner, S., Helmreich, I., Dahmen, N., Lieb, K., \& Tadić, A. (2011). Reliability of three alternate forms of the trail making tests A and B. Archives of Clinical Neuropsychology, 26(4), 314-321. doi:10.1093/arclin/acr024

Walter, T., Hale, L., \& Smith, C. (2015). Blue prescription: A single-subject design intervention to enable physical activity for people with stroke. International Journal of Therapy and Rehabilitation, 22(2), 87-95.

Wang, Q., Ying Chair, S., Mi Ling Wong, E., \& Li, X. (2016). The effects of music intervention on sleep quality in community-dwelling elderly. Journal of Alternative and Complementary Medicine, 22(7), 576-584. doi:10.1089/acm.2015.0304

Watson, M., Garden, J., Swedlove, F. \& Brown, C. A. (2014). Back to the basics: Sleep and occupation. Occupational Therapy Now, 16(6), 8-10.

Weaver, T. E., Laizner, A. M., Evans, L. K., Maislin, G., Chugh, D. K., Lyon, K.,...Dinges, D. F. (1997). An instrument to measure functional status outcomes for disorders of excessive sleepiness. Sleep, 20(10), 835-843. 
Wheaton, A. G., Liu, Y., Perry, G. S., \& Croft, J. B. (2011). Effect of short sleep duration on daily activities-United States, 2005-2008. Morbidity and Mortality Weekly Report, 60(8), $239-242$.

Wilcox, A. A. (1998). An occupational perspective of health. Thorofare, NJ: SLACK. XXX. (2019). Physical therapy and rehabilitation: Community re-entry program.

Retrieved from http://www.sharp.com/services/rehab/community-reentry.cfm 
Appendix A

Trail Making Test, Part B Score Sheet

\section{Trail Making Test (TMT) Parts A \& B}

\section{Instructions:}

Both parts of the Trail Making Test consist of 25 circles distributed over a sheet of paper. In Part A. the circles are numbered $1-25$, and the patient should draw lines to connect the numbers in ascending order. In Part $B$, the circles include both numbers $(1-13)$ and letters $(A-L)$; as in Part $A$, the patient draws lines to connect the circles in an ascending pattern, but with the added task of alternating between the numbers and letters (i.e., 1-A-2-B-3-C, etc.). The patient should be instructed to connect the circles as quickly as possible, without lifting the pen or pencil from the paper. Time the patient as he or she connects the "trail." If the patient makes an error, point it out immediately and allow the patient to correct it. Errors affect the patient's score only in that the correction of errors is included in the completion time for the task. It is unnecessary to continue the test if the patient has not completed both parts after five minutes have elapsed.

Step 1: Give the patient a copy of the Trail Making Test Part A worksheet and a pen or pencil.

Step 2: Demonstrate the test to the patient using the sample sheet (Trail Making Part A SAMPLE).

Step 3: Time the patient as he or she follows the "trail" made by the numbers on the test.

Step 4: Record the time.

Step 5: $\quad$ Repeat the procedure for Trail Making Test Part B.

\section{Scoring:}

Results for both TMT A and B are reported as the number of seconds required to complete the task; therefore, higher scores reveal greater impairment.

$\begin{array}{llll} & \text { Average } & \text { Deficient } & \text { Rule of Thumb } \\ \text { Trail A } & 29 \text { seconds } & >78 \text { seconds } & \text { Most in } 90 \text { seconds } \\ \text { Trail B } & 75 \text { seconds } & >273 \text { seconds } & \text { Most in } 3 \text { minutes }\end{array}$

\section{Sources:}

- Corrigan JD, Hinkeldey MS. Relationships between parts A and B of the Trail Making Test. $J$ Clin Psychol. 1987;43(4):402-409.

- Gaudino EA, Geisler MW, Squires NK. Construct validity in the Trail Making Test: what makes Part B harder? J Clin Exp Neuropsychol. 1995:17(4):529-535.

- Lezak MD, Howieson DB, Loring DW. Neuropsychological Assessment. 4th ed. New York: Oxford University Press; 2004.

- Reitan RM. Validity of the Trail Making test as an indicator of organic brain damage. Percept Mot Skills. 1958:8:271-276. 


\section{Trail Making Test Part B}

Patient's Name:

Date:

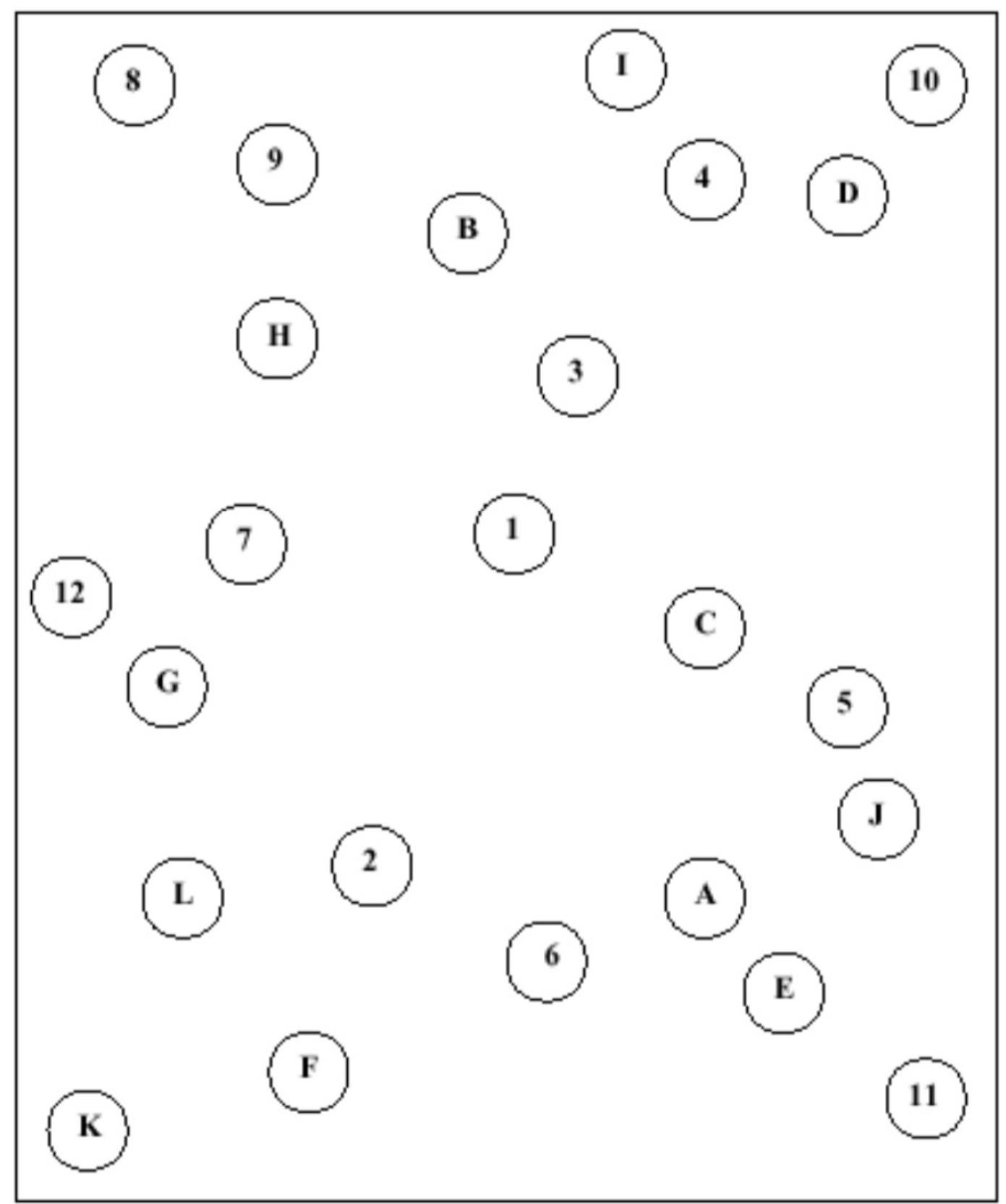


Appendix B

Insomnia Severity Index Score Sheet

\section{Insomnia Severity Index (ISI)}

\section{Subject ID:}

Date:

For each question below, please circle the number corresponding most accurately to your sleep patterns in the LAST MONTH.

For the first three questions, please rate the SEVERITY of your sleep difficulties.

1. Difficulty falling asleep:

\begin{tabular}{ccccc} 
None & Mild & Moderate & Severe & Very Severe \\
\hline 0 & 1 & 2 & 3 & 4
\end{tabular}

2. Difficulty staying asleep:

\begin{tabular}{ccccc} 
None & Mild & Moderate & Severe & Very Severe \\
\hline 0 & 1 & 2 & 3 & 4
\end{tabular}

3. Problem waking up too early in the morning:

\begin{tabular}{ccccc} 
None & Mild & Moderate & Severe & Very Severe \\
\hline 0 & 1 & 2 & 3 & 4
\end{tabular}

4. How SATISFIED/dissatisfied are you with your current sleep pattern?

\begin{tabular}{ccccc}
$\begin{array}{c}\text { Very } \\
\text { Satisfied }\end{array}$ & Satisfied & Neutral & Dissatisfied & $\begin{array}{c}\text { Very } \\
\text { Dissatisfied }\end{array}$ \\
\hline 0 & 1 & 2 & 3 & 4
\end{tabular}

5. To what extent do you consider your sleep problem to INTERFERE with your daily functioning (e.g., daytime fatigue, ability to function at work/daily chores, concentration, memory, mood).

Not at all A Little Somewhat Much Very Much Interfering Interfering Interfering Interfering Interfering

\begin{tabular}{lllll}
\hline 0 & 1 & 2 & 3 & 4
\end{tabular}

6. How NOTICEABLE to others do you think your sleeping problem is in terms of impairing the quality of your life?

$\begin{array}{cccc}\text { Not at all A little } & \text { Somewhat } & \text { Much } & \text { Very Much } \\ \text { Noticeable Noticeable } & \text { Noticeable } & \text { Noticeable } & \text { Noticeable }\end{array}$

$\begin{array}{lllll}0 & 1 & 2 & 3 & 4\end{array}$

7. How WORRIED/distressed are you about your current sleep problem? Not at all A Little Somewhat Much Very Much

\begin{tabular}{lllll}
\hline 0 & 1 & 2 & 3 & 4
\end{tabular}

(C) Morin, C.M. (1993, 1996, 2000, 2006). 


\section{Appendix C}

\section{Sleep Hygiene Index Score Sheet}

Sleep Hygiene Index University of Arkansas at Little Rock $\bullet$ Biobehavioral Laboratory

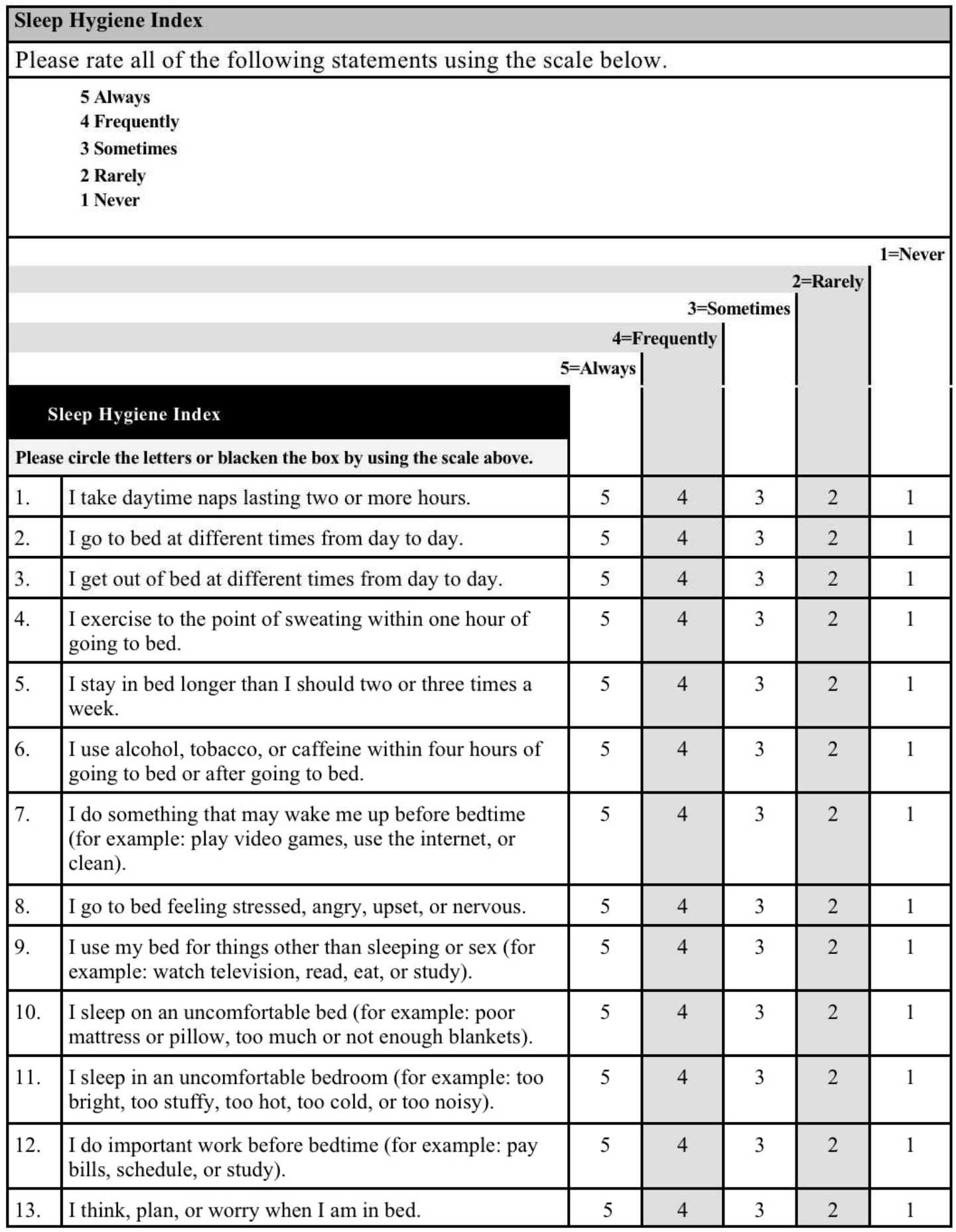




\title{
Appendix D
}

\author{
Functional Outcomes of Sleep Questionnaire Score Sheet
}

\section{FUNCTIONAL OUTCOMES OF SLEEP QUESTIONNAIRE (FOSQ)}

\author{
Scoring Instructions September 1996 Version \\ (Revised 7/18/00)
}

$\begin{array}{lll}\underline{\text { Subscales }} & \underline{\text { \# Questions }} & \underline{\underline{\text { Item \# }}} \\ \text { General Productivity } & \text { 8 questions } & 1-4,8-11 \\ \text { Social Outcome } & 2 \text { questions } & 12,13 \\ \text { Activity Level } & 9 \text { questions } & 5,14-16,22-26 \\ \text { Vigilance } & 7 \text { questions } & 6,7,17-21 \\ \text { Intimate Relationships and Sexual Activity } & 4 \text { questions } & 27-30\end{array}$

Subscale Scores: A response score of 0 for an item should be coded as a N/A or missing response. Thus, the potential range of scores for any item is $1-4$. Calculate the mean of the answered items with responses equal to or greater than 1 for each subscale. This is the weighted mean item total or subscale score. For example, if a subscale has six questions, and one question has a missing response and one with a N/A response, then you would not include those two questions when you added the responses and you would divide by four instead of six when calculating the mean. This prevents a score bias due to missing answers or skipped questions because an individual does not engage in a particular activity do to reasons other than disorders of excessive sleepiness. The potential range of scores for each subscale is $1-4$.

To obtain a Total Score: Take all of the subscale scores and calculate the mean of these scores and then multiply that mean by five. Multiply by five regardless of the number of subscales scores used in the computation of the mean. For example, if you a subscale score for all subscales, then you multiply the mean of those scores by 5 ; if you have subscale scores for only 4 of the 5 subscales, then you would also multiply the mean by five. The potential range of scores for the Total Score is 5 $-20$. 
Site:

ID \#:

Date of Data Entry: __ Trial

Name:

Date:

\section{FUNCTIONAL OUTCOMES OF SLEEP QUESTIONNAIRE (FOSQ)}

Some people have difficulty performing everyday activities when they feel tired or sleepy. The purpose of this questionnaire is to find out if you generally have difficulty carrying out certain activities because you are too sleepy or tired. In this questionnaire, when the words "sleepy" or "tired" are used, it means the feeling that you can't keep your eyes open, your head is droopy, that you want to "nod off", or that you feel the urge to take a nap. These words do not refer to the tired or fatigued feeling you may have after you have exercised.

DIRECTIONS: Please put a ( $\_$) in the box for your answer to each question. Select only one answer for each question. Please try to be as accurate as possible. All information will be kept confidential.

\begin{tabular}{|c|c|c|c|c|}
\hline$(0)$ & $(4)$ & $(3)$ & $(2)$ & $(1)$ \\
I don't do & No & Yes, & Yes, & Yes, \\
this & difficulty & a little & moderate \\
difficulty & difficulty & difficulty \\
activity & & & & \\
for other & & & & \\
reasons & & & & \\
\hline
\end{tabular}

1.Do you have difficulty concentrating on the things you do because you are sleepy or tired?

2.Do you generally have difficulty remembering things, because you are sleepy or tired?

3.Do you have difficulty finishing a meal because you become sleepy or tired?

4.Do you have difficulty working on a hobby (for example, sewing, collecting, gardening) because

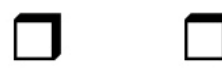
you are sleepy or tired? 
Site:

Date of Data Entry:

Name:
ID \#:

Trial

Date:

\begin{tabular}{|c|c|c|c|c|}
\hline $\begin{array}{c}(0) \\
\text { I don't do } \\
\text { this }\end{array}$ & $\begin{array}{c}\text { No } \\
\text { difficulty }\end{array}$ & $\begin{array}{c}\text { Yes, } \\
\text { a little } \\
\text { difficulty }\end{array}$ & $\begin{array}{c}\text { Yes, } \\
\text { moderate } \\
\text { difficulty }\end{array}$ & $\begin{array}{c}\text { Yes, } \\
\text { extreme } \\
\text { difficulty }\end{array}$ \\
$\begin{array}{c}\text { for other } \\
\text { reasons }\end{array}$ & & & & \\
\hline
\end{tabular}

5.Do you have difficulty doing work around the house (for example, cleaning house, doing laundry, taking out the trash, repair work) because you are sleepy or tired?

6.Do you have difficulty operating a motor vehicle for short distances (less than 100 miles) because you become sleepy or tired?

7.Do you have difficulty operating a motor vehicle for long distances (greater than 100 miles) because you become sleepy or tired?

8.Do you have difficulty getting things done because you are too sleepy or tired to drive or take public transportation?

9.Do you have difficulty taking care of financial affairs and doing paperwork (for example,

$\square \quad \square$
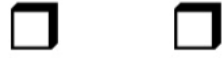
writing checks, paying bills, keeping financial records, filling out tax forms, etc.) because you are sleepy or tired? 
Site:

Date of Data Entry:

Name:
ID \#:

Trial

Date:

\begin{tabular}{|c|c|c|c|c|}
\hline$(0)$ & $(4)$ & $(3)$ & $(2)$ & $(1)$ \\
$\begin{array}{c}\text { I don't do } \\
\text { this }\end{array}$ & $\begin{array}{c}\text { No } \\
\text { difficulty }\end{array}$ & $\begin{array}{c}\text { Yes, } \\
\text { a little } \\
\text { difficulty }\end{array}$ & $\begin{array}{c}\text { Yes, } \\
\text { moderate } \\
\text { difficulty }\end{array}$ & $\begin{array}{c}\text { Yes, } \\
\text { extreme } \\
\text { difficulty }\end{array}$ \\
$\begin{array}{c}\text { activity } \\
\text { for other } \\
\text { reasons }\end{array}$ & & & & \\
\hline
\end{tabular}

10.Do you have difficulty performing employed or volunteer work because you are sleepy or tired?

11. Do you have difficulty maintaining a telephone conversation, because you become sleepy or tired?

12. Do you have difficulty visiting with your family or friends in your home because you become sleepy or tired?

13. Do you have difficulty visiting with your family or friends in their home because you become sleepy or tired?

14. Do you have difficulty doing things for your family or friends because you are too sleepy or tired?
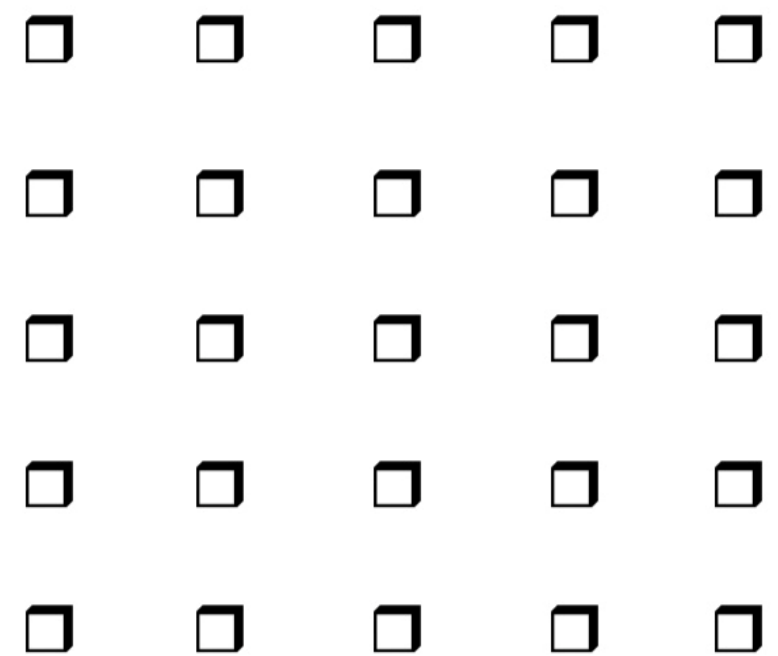

\begin{tabular}{|c|c|c|c|}
\hline $\begin{array}{l}\text { (4) } \\
\text { No }\end{array}$ & $\begin{array}{l}(3) \\
\text { Yes, } \\
\text { a little }\end{array}$ & $\begin{array}{c}(2) \\
\text { Yes, } \\
\text { moderately }\end{array}$ & $\begin{array}{c}(1) \\
\text { Yes, } \\
\text { extremely }\end{array}$ \\
\hline
\end{tabular}

15. Has your relationship with family, friends or work colleagues been affected because you are $\square \quad \square$
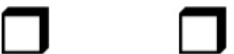
sleepy or tired?

In what way has your relationship been affected?

CWeaver, September 1996

Functional Outcomes of Sleep Questionnaire (FOSQ)

fosq.97 updated 11/98

Page 3 
Site:

Date of Data Entry:

Name:

ID \#:

Trial

Date:

\begin{tabular}{|c|c|c|c|c|}
\hline$(0)$ & $(4)$ & $(3)$ & $(2)$ & $(1)$ \\
$\begin{array}{c}\text { I don't do } \\
\text { this } \\
\text { activity } \\
\text { for other } \\
\text { reasons }\end{array}$ & $\begin{array}{c}\text { No } \\
\text { difficulty }\end{array}$ & $\begin{array}{c}\text { Yes, } \\
\text { a little } \\
\text { difficulty }\end{array}$ & $\begin{array}{c}\text { Yes, } \\
\text { moderate } \\
\text { difficulty }\end{array}$ & $\begin{array}{c}\text { Yes, } \\
\text { extreme } \\
\text { difficulty }\end{array}$ \\
\hline
\end{tabular}

16. Do you have difficulty exercising or participating in a sporting activity because you are too sleepy or tired?

17. Do you have difficulty watching a movie or videotape because you become sleepy or tired?

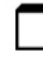

18. Do you have difficulty enjoying the theater or a lecture because you become sleepy or tired?

19. Do you have difficulty enjoying a concert because you become sleepy or tired?

20. Do you have difficulty watching TV because you are sleepy or tired?

21. Do you have difficulty participating in religious services, meetings or a group or club, because you are sleepy or tired?

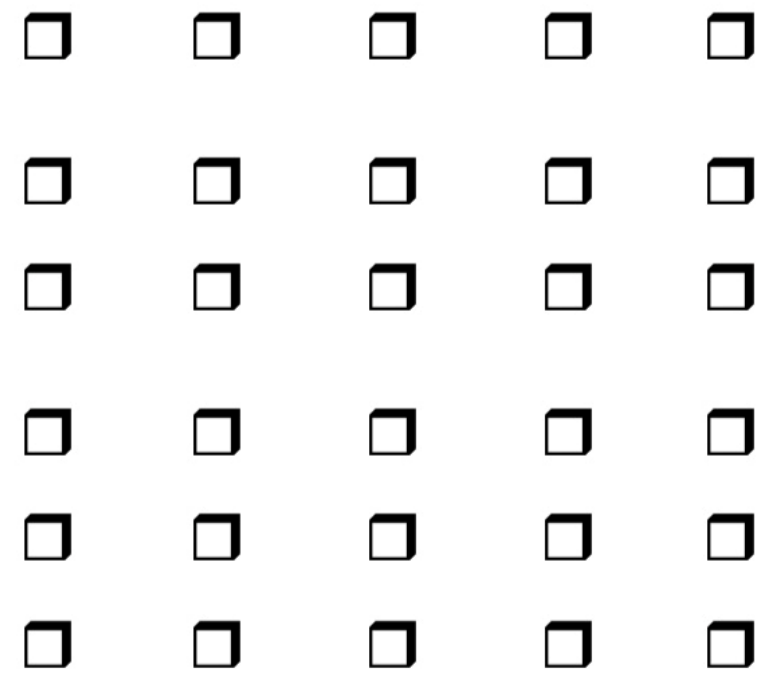


Site:

Date of Data Entry:

Name:
ID \#:

Trial

Date:

\begin{tabular}{|c|c|c|c|c|}
\hline$(0)$ & $(4)$ & $(3)$ & $(2)$ & $(1)$ \\
$\begin{array}{c}\text { I don't do } \\
\text { this }\end{array}$ & $\begin{array}{c}\text { No } \\
\text { difficulty }\end{array}$ & $\begin{array}{c}\text { Yes, } \\
\text { a little } \\
\text { difficulty }\end{array}$ & $\begin{array}{c}\text { Yes, } \\
\text { moderate } \\
\text { difficulty }\end{array}$ & $\begin{array}{c}\text { Yes, } \\
\text { extreme } \\
\text { difficulty }\end{array}$ \\
$\begin{array}{c}\text { for other } \\
\text { reasons }\end{array}$ & & & & \\
\hline
\end{tabular}

22. Do you have difficulty being as active as you want to be in the evening because you are sleepy or tired?

23. Do you have difficulty being as active as you want to be in the morning because you are sleepy or tired?

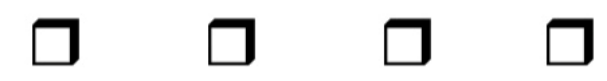

(0)

I don't do this for other reasons
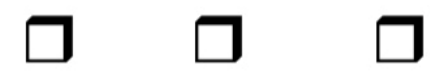

\begin{tabular}{|c|c|c|c|c|}
\hline$(0)$ & $(4)$ & $(3)$ & $(2)$ & $(1)$ \\
$\begin{array}{c}\text { I don't do this } \\
\text { for other } \\
\text { reasons }\end{array}$ & $\begin{array}{c}\text { No } \\
\text { difficulty }\end{array}$ & $\begin{array}{c}\text { Yes, } \\
\text { a } \\
\text { little } \\
\text { difficulty }\end{array}$ & $\begin{array}{c}\text { Yes, } \\
\text { moderate } \\
\text { difficulty }\end{array}$ & $\begin{array}{c}\text { Yes, } \\
\text { extreme } \\
\text { difficulty }\end{array}$ \\
\hline
\end{tabular}

24. Do you have difficulty being as active as you want to be in the afternoon because you are sleepy or tired?

25. Do you have difficulty keeping pace with others your own age because you are sleepy or tired?

\begin{tabular}{|c|c|c|c|}
\hline$(1)$ & $(2)$ & $(3)$ & $(4)$ \\
Very Low & Low & Medium & High \\
\hline
\end{tabular}

26. How would you rate your general level of activity?

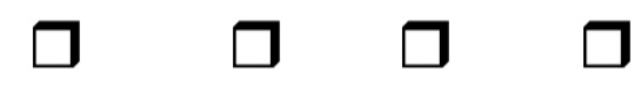


Site:

Date of Data Entry:

Name:
ID \#:

Trial

Date:
27. Has your intimate or sexual relationship been affected because you are sleepy or tired?

28. Has your desire for intimacy or sex been affected because you are sleepy or tired?

29. Has your ability to become sexually aroused been affected because you are sleepy or tired?

30. Has your ability to "come" (have an orgasm) been affected because you are sleepy or tired?

$\begin{array}{cccc}(4) & (3) & (2) & (1) \\ \text { No } & \text { Yes, } & \text { Yes, } & \text { Yes, } \\ & \text { a } & \text { moderately } & \text { extremely }\end{array}$
I don't engage in sexual activity for other reasons
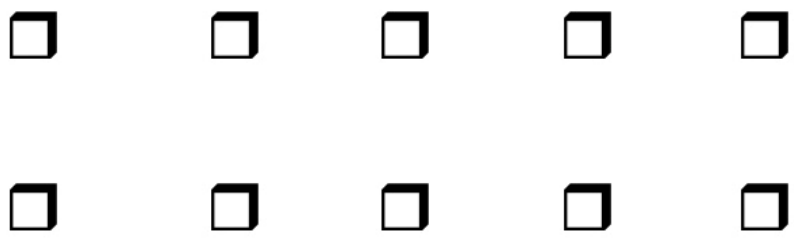

$\square$
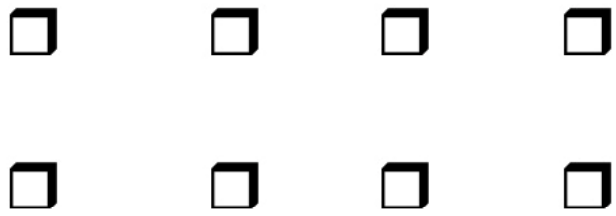
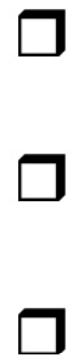

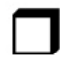<smiles>C1CCC1</smiles><smiles>C1CCCC1</smiles>

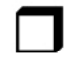

Thank you for completing this questionnaire. 


\section{Appendix E}

\section{Epworth Sleepiness Scale Score Sheet}

\section{Epworth Sleepiness Scale}

Name:

Today's date:

Your age (yrs):

Your gender $($ Male $=\mathrm{M}$, Female $=\mathrm{F})$ :

How likely are you to doze off or fall asleep in the following situations, in contrast to just feeling tired?

This refers to your usual way of life recently.

Even if you haven't done some of these things recently, try to figure out how they would have affected you.

Use the following scale to choose the most appropriate number for each situation:

$$
\begin{aligned}
& 0=\text { no chance of dozing } \\
& 1=\text { slight chance of dozing } \\
& 2=\text { moderate chance of dozing } \\
& 3=\text { high chance of dozing }
\end{aligned}
$$

It is important that you answer each item as best as you can.

\section{Situation}

Sitting and reading

Watching TV

Sitting inactive in a public place (e.g., a theater or a meeting)

As a passenger in a car for an hour without a break

Lying down to rest in the afternoon when circumstances permit

Sitting and talking to someone

Sitting quietly after a lunch without alcohol

In a car or bus, while stopped for a few minutes in traffic
Chance of Dozing (0-3)

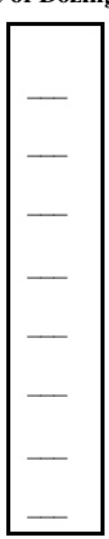

THANK YOU FOR YOUR COOPERATION

ESS C MW Johns 1990-1997. Used under License 


\title{
Appendix F
}

\section{Consensus Sleep Diary Worksheet}

\author{
Sleep Diary Instructions - Core
}

\section{General Instructions}

What is a Sleep Diary? A sleep diary is designed to gather information about your daily sleep pattern.

How often and when do I fill out the sleep diary? It is necessary for you to complete your sleep diary every day. If possible, the sleep diary should be completed within one hour of getting out of bed in the morning.

What should I do if I miss a day? If you forget to fill in the diary or are unable to finish it, leave the diary blank for that day.

What if something unusual affects my sleep or how I feel in the daytime? If your sleep or daytime functioning is affected by some unusual event (such as an illness, or an emergency) you may make brief notes on your diary.

What do the words "bed" and "day" mean on the diary? This diary can be used for people who are awake or asleep at unusual times. In the sleep diary, the word "day" is the time when you choose or are required to be awake. The term "bed" means the place where you usually sleep.

Will answering these questions about my sleep keep me awake? This is not usually a problem. You should not worry about giving exact times, and you should not watch the clock. Just give your best estimate.

\section{Item Instructions}

Use the guide below to clarify what is being asked for each item of the Sleep Diary.

Date: Write the date of the morning you are filling out the diary.

1. What time did you get into bed? Write the time that you got into bed. This may not be the time that you began "trying" to fall asleep.

2. What time did you try to go to sleep? Record the time that you began "trying" to fall asleep.

3. How long did it take you to fall asleep? Beginning at the time you wrote in question 2, how long did it take you to fall asleep.

4. How many times did you wake up, not counting your final awakening? How many times did you wake up between the time you first fell asleep and your final awakening?

5. In total, how long did these awakenings last? What was the total time you were awake between the time you first fell asleep and your final awakening. For example, if you woke 3 times for 20 minutes, 35 minutes, and 15 minutes, add them all up $(20+35+15=70 \mathrm{~min}$ or $1 \mathrm{hr}$ and $10 \mathrm{~min})$.

6. What time was your final awakening? Record the last time you woke up in the morning.

7. What time did you get out of bed for the day? What time did you get out of bed with no further attempt at sleeping? This may be different from your final awakening time (e.g. you may have woken up at 6:35 a.m. but did not get out of bed to start your day until 7:20 a.m.)

8. How would you rate the quality of your sleep? "Sleep Quality" is your sense of whether your sleep was good or poor.

9. Comments If you have anything that you would like to say that is relevant to your sleep feel free to write it here. 


\begin{tabular}{|c|c|c|c|c|c|c|c|c|}
\hline \multicolumn{3}{|c|}{ Sample } & \multicolumn{2}{|c|}{ Consensus Sleep Diary-Core } & \multicolumn{2}{|c|}{ ID/Name: } & & \\
\hline Today's date & $4 / 5 / 11$ & & & & & & & \\
\hline $\begin{array}{l}\text { 1. What time did } \\
\text { you get into bed? }\end{array}$ & 10:15 p.m & & & & & & & \\
\hline $\begin{array}{l}\text { 2. What time did } \\
\text { you try to go to } \\
\text { sleep? }\end{array}$ & 11:30 p.m & & & & & & & \\
\hline $\begin{array}{l}\text { 3. How long did it } \\
\text { take you to fall } \\
\text { asleep? }\end{array}$ & $55 \mathrm{~min}$. & & & & & & & \\
\hline $\begin{array}{l}\text { 4. How many } \\
\text { times did you } \\
\text { wake up, not } \\
\text { counting your final } \\
\text { awakening? }\end{array}$ & 3 times & & & & & & & \\
\hline $\begin{array}{l}\text { 5. In total, how } \\
\text { long did these } \\
\text { awakenings last? }\end{array}$ & $\begin{array}{l}1 \text { hour } \\
10 \mathrm{~min} \text {. }\end{array}$ & & & & & & & \\
\hline $\begin{array}{l}\text { 6. What time was } \\
\text { your final } \\
\text { awakening? }\end{array}$ & 6:35 a.m. & & & & & & & \\
\hline $\begin{array}{l}\text { 7. What time did } \\
\text { you get out of bed } \\
\text { for the day? }\end{array}$ & 7:20 a.m & & & & & & & \\
\hline $\begin{array}{l}\text { 8. How would you } \\
\text { rate the quality of } \\
\text { your sleep? }\end{array}$ & $\begin{array}{l}\square \text { Very poor } \\
\square \text { Poor } \\
\square \text { Fair } \\
\square \text { Good } \\
\square \text { Very good } \\
\end{array}$ & $\begin{array}{l}\square \text { Very poor } \\
\square \text { Poor } \\
\square \text { Fair } \\
\square \text { Good } \\
\square \text { Very good }\end{array}$ & $\begin{array}{l}\square \text { Very poor } \\
\square \text { Poor } \\
\square \text { Fair } \\
\square \text { Good } \\
\square \text { Very good }\end{array}$ & $\begin{array}{l}\square \text { Very poor } \\
\square \text { Poor } \\
\square \text { Fair } \\
\square \text { Good } \\
\square \text { Very good }\end{array}$ & $\begin{array}{l}\square \text { Very poor } \\
\square \text { Poor } \\
\square \text { Fair } \\
\square \text { Good } \\
\square \text { Very good }\end{array}$ & $\begin{array}{l}\square \text { Very poor } \\
\square \text { Poor } \\
\square \text { Fair } \\
\square \text { Good } \\
\square \text { Very good }\end{array}$ & $\begin{array}{l}\square \text { Very poor } \\
\square \text { Poor } \\
\square \text { Fair } \\
\square \text { Good } \\
\square \text { Very good }\end{array}$ & $\begin{array}{l}\square \text { Very poor } \\
\square \text { Poor } \\
\square \text { Fair } \\
\square \text { Good } \\
\square \text { Very good }\end{array}$ \\
\hline $\begin{array}{l}\text { 9. Comments } \\
\text { (if applicable) }\end{array}$ & I have a cold & & & & & & & \\
\hline
\end{tabular}




\section{Appendix G}

\section{Sample Fitbit Data Output}

\begin{tabular}{|c|c|c|c|c|c|c|c|c|}
\hline \multicolumn{9}{|l|}{ Sleep } \\
\hline Start Time & End Time & Minutes Asleep & Minutes Awake & Number of Awakenings & Time in Bed & Minutes REM Sleep & Minutes Light Sleep & Minutes Deep Sleep \\
\hline 2019-04-25 9:10PM & 2019-04-26 6:17AM & 453 & 94 & 38 & 547 & 75 & 312 & 66 \\
\hline 2019-04-23 10:21PM & 2019-04-24 6:22AM & 432 & 49 & 42 & 481 & 49 & 271 & 112 \\
\hline 2019-04-22 11:09PM & 2019-04-23 7:35AM & 429 & 77 & 25 & 506 & 57 & 291 & 81 \\
\hline 2019-04-20 10:48PM & 2019-04-21 6:13AM & 401 & 44 & 30 & 445 & 43 & 280 & 78 \\
\hline 2019-04-18 8:52PM & 2019-04-19 4:05AM & 364 & 69 & 24 & 433 & 43 & 264 & 57 \\
\hline 2019-04-17 11:23PM & 2019-04-18 7:01AM & 407 & 51 & 33 & 458 & 69 & 289 & 49 \\
\hline 2019-04-16 9:17PM & 2019-04-17 6:16АM & 476 & 63 & 42 & 539 & 77 & 284 & 115 \\
\hline 2019-04-15 9:07PM & 2019-04-16 7:24AM & 542 & 75 & 36 & 617 & 142 & 295 & 105 \\
\hline 2019-04-14 10:49PM & 2019-04-15 5:55AM & 383 & 43 & 33 & 426 & 66 & 255 & 62 \\
\hline 2019-04-13 11:05PM & 2019-04-14 5:51AM & 360 & 46 & 27 & 406 & 86 & 220 & 54 \\
\hline
\end{tabular}




\section{Appendix $\mathrm{H}$}

\section{Semi-structured Interview Guide}

1. Tell me about your typical routine when you are getting ready for bed. (Probes: pre/post injury, pre/post study, evening activities and routines, food/liquid consumption, certain activities that might affect sleep).

2. What is your idea of a good night of sleep? (Probes: number of hours, number of awakenings, total time to fall asleep, the meaning of adequate sleep, how you feel in the morning).

3. Tell me how getting a good night of sleep influences your daily activities or routines the following day (Probes: ADLs, work, leisure pursuits, fatigue and daytime sleepiness).

4. How does a bad night of sleep influence your daily activities or routines the following day? (Probes: ADLs, work, leisure pursuits, fatigue and daytime sleepiness).

5. Tell me about the parts of sleep that are difficult for you? (Probes: falling asleep, staying asleep, multiple awakenings, how you feel).

6. What kinds of things make the most difference in you being able to get a good night of sleep? (Probes: personal factors - pain/discomfort, ability to quiet the mind, environmental factors, occupational factors/activities, routines and habits). 
Appendix I

Participant Consent Form

General Informed Consent Form

NSU Consent to be in a Research Study Entitled

Implications of a Multicomponent Sleep Program after Brain Injury: A Mixed Methods Study

Who is doing this study?

College: Dr. Pallavi Patel College of Health Care Sciences, Department of Occupational

Therapy

Principal Investigator: Brandi M. Fulwider, MS in Occupational Therapy

Faculty Advisor/Dissertation Chair: Wendy Stav, PhD

Site Information: XXX's Community Re-entry Program

Funding: Unfunded

\section{What is this study about?}

This is a research study that will test and create new ideas that other people can use. The goal of this study is improved sleep after brain injury and to understand how sleep affects daily life. This study has many potential benefits. One benefit will be learning about healthy sleep habits.

\section{Why are you asking me to be in this study?}

You are a member of the community re-entry program. You also reported sleep problems.

This study will have about 3-6 people. Everyone will be from the community re-entry program. You will do a sleep interview and/or be in the sleep program.

\section{What will I be doing if I agree to be in this study?}

You will do sleep tests and learn about the study at the first two meetings. The first two meetings will be about one hour each. You will talk about your sleep during an interview. You will also have two meetings every week for $4-5$ weeks in the sleep program. These meetings will be about 15-30 minutes each. There will be a telephone call to you one month after the sleep program is done. This call will be about 10 minutes long.

You will be in the study while you are in the community re-entry program. The researcher will look at your medical chart to make sure you should be in this study.

Research Study Information:

To be in this study you have to read, write, understand, and talk in English. You also need a family member that can help you at home during the study. You need to have sleep and cognitive problems and need to wear a sleep tracker on the wrist during sleep. You need to finish all parts of the study. The study includes a sleep interview, a sleep program, sleep tests, and a phone call in one month after the study.

The first two meetings will have four sleep tests and a recorded interview. You will also learn about the study and about sleep. The first four tests will take about 25 minutes. The first two 
meetings will be about one hour. Other meetings will be two times per week. You will learn about the sleep program, how to record sleep, turn in forms, and talk to the researcher during meetings. Weekly meetings will be about 15-30 minutes. You will do two sleep tests every week. These tests will take about 15-20 minutes. You will need to record your sleep information every morning during the study. This will take about 5-10 minutes every day. The last meeting will have three sleep tests. This may take about 30 minutes. Some meetings can be during your lunch break. Everyone in the study will do the same sleep interview and/or the sleep program. You will get a phone call for a sleep test one month after the sleep program ends.

\section{Could I be removed from the study early by the researcher?}

You could be taken out of the study early if you do not meet the rules to be in the study. You could also be taken out of the study if you do not wear the sleep tracker, do not fill out the sleep forms, do not do sleep tests, do not bring the Fitbit to meetings, or if do not come to meetings.

\section{Are there possible risks and discomforts to me?}

This study will have minimal risk to you. The things you will do will likely have no more risk of harm than everyday life.

- Physical risks: You may feel sleepy sometimes during the study. This risk is minimal and could happen without being in this study. You should always be careful when driving or using heavy machinery if you feel sleepy.

- Psychological risks: You may learn that sleep problems can affect daily life and health by being in this study. You will also learn how to address your sleep problems.

You may find some questions we ask you (or some things we ask you to do) to be upsetting or stressful. You can talk to someone who can help you with these feelings if that happens. The community re-entry program has counseling if you need it when you are in the program.

\section{What happens if I do not want to be in this study?}

You do not have to be in this study and can leave at any time. You will not be punished or lose any services if you leave or if you do not want to be in the study anymore. If you want to leave the study, any information collected about you before the date you leave the study will be kept in the study records for 36 months from the end of the study. You can ask that the information about you not be used.

\section{What if there is new information learned during the study that may affect my decision to stay in the study? \\ If we learn important new information about the study, we will give this information to you. You may have to sign a new Informed Consent Form.}

\section{Are there any benefits for being in this study?}

This study can help your sleep and sleep habits. You may also be able to keep the Fitbit from the study if you finish both the sleep program and the sleep interview. You may also have no benefit from this study. I hope that the information learned from this study will help other people with sleep problems in the future. 


\section{Will I be paid or be given compensation for being in the study?}

You will not get any payment for this study. You may be able to keep the Fitbit after all information is collected for the study. You must give the Fitbit back to the researcher right away if you do not finish all of the study.

\section{Will it cost me anything?}

There are no costs to be in this study.

\section{How will you keep my information private?}

All information about you will be private. We will protect your identity with the use of codes and no real names. The researcher, the Institutional Review Board, and other members of this institution, and any regulatory agencies can review this information. We will not identify you in any papers or reports from the study. Your private information will be kept on the researcher's password-protected computer and in a locked filing cabinet for 36 months from the end of the study. We will destroy it after that by shredding all documents and erasing all computer files.

\section{Will there be any Audio or Video Recording?}

This study uses a voice recording for the sleep interview. The researcher, the Institutional Review Board, and other members of this institution can listen to this recording. The recording will be private and will be erased after 36 months. The recording will have information about you on it. The researcher will try to keep other people from listening to it.

\section{Whom can I contact with questions, concerns, comments, or complaints?}

You can ask me any questions now or later. If you have more questions about the research, your research rights, or have a research-related injury, please contact:

Primary contact:

Brandi Fulwider, MS in Occupational Therapy, can be reached at bf615@mynsu.nova.edu

If primary is not available, contact:

Dr. Wendy Stav, PhD can be reached at ws133@nova.edu.

\section{Research Participants Rights}

For questions/concerns regarding your research rights, please contact:

Institutional Review Board

Nova Southeastern University

(954) 262-5369 / Toll Free: 1-866-499-0790

IRB@nova.edu

You may also visit the NSU IRB website at www.nova.edu/irb/information-for-researchparticipants for more information about your rights in this study.

XXX Institutional Review Board

San Diego, CA 92123 


\section{Research Consent \& Authorization Signature Section}

Voluntary Participation - You are not required to participate in this study. In the event you do participate, you may leave this research study at any time. If you leave this research study before it is completed, there will be no penalty to you, and you will not lose any benefits to which you are entitled.

If you agree to participate in this research study, sign this section. You will be given a signed copy of this form to keep. You do not waive any of your legal rights by signing this form.

SIGN THIS FORM ONLY IF THE STATEMENTS LISTED BELOW ARE TRUE:

- You have read the above information.

- Your questions have been answered to your satisfaction about the research.

\section{Adult Signature Section}

I have voluntarily decided to take part in this research study. 
Appendix J

Health Insurance Portability and Accountability Act Form

\section{Authorization to Use your Protected Health Information (PHI)}

Study Title: Implications of a Multicomponent Sleep Program after Brain Injury: A Mixed Methods Study

\section{Investigator: Brandi Fulwider, MS, OTR/L}

San Diego, CA 92123

bf615@mynsu.nova.edu

Protected Health Information, or PHI, is any personal health information through which you can be identified. We are asking for your permission to use your PHI in this research study. The information we may use includes your past and present health information, information that can be used to contact you, and results of your medical tests. The specific items of information that will be used and disclosed include:

- $\quad$ XXX's Community Re-entry Program occupational therapy notes and neuropsychology cognitive testing results

The following people will access, use or disclose your PHI for the purpose of this research:

- Brandi Fulwider, Occupational Therapist, of Nova Southeastern University and XXX Hospital

\section{Who will see my PHI?}

- the XXX Institutional Review Board

- the Nova Southeastern University Institutional Review Board

\section{How long will Brandi Fulwider use and share my information, and what will} it be used for?

- The PHI will be used to determine if you are eligible to participate in her research study.

- This permission to release your PHI expires December 31, 2019. Research reports can be used forever.

- After disclosure by the researcher, your PHI leaves the control of XXX. That means that your PHI may be shared with other entities and may no 
longer be protected by the privacy laws and regulations that protect such information normally.

What if I decide not to share my PHI anymore and want to withdraw my authorization for the use and disclosure of my PHI for this study?

- You must write to the researcher and tell her that you no longer want to share your information. Write to the researcher at: Brandi Fulwider bf615@mynsu.nova.edu

- You will no longer be a part of the research study because the researcher will not be able to use any new information about you.

- The researcher can continue to use any of the PHI that they already have.

- You will still get the same medical care that you have always had from XXX.

Do I have the right to see and receive a copy of my research information?

You can see your research information if:

- it is also being used for your current treatment, or

- at the end of the study.

$* * * * * * * * * * * * * * * * * * * * * * * * * * * * * * * * * * * * * * * * * * * * * * * * * * * * * * * * * * * * * * * * * *$ 


\section{Authorization:}

If you agree to share your PHI, you must sign this form below. If you do not sign this form, you will not be able to participate in the research study. You will be given a copy of this form.

Signature of Participant

Printed Name

Date

Signature of

Printed Name

Date

Principal Investigator

If the participant is not competent to provide informed consent, please sign as appropriate:

\begin{tabular}{l}
\hline $\begin{array}{l}\text { Signature of Durable Power of Attorney } \\
\text { for Health Care }\end{array}$ \\
Or
\end{tabular}

Signature of Legally Authorized Representative Printed Name Date

This document is available on IRBANA or by contacting research@sharp.com. 
Appendix K

Demographic Data Form

\section{Client Demographic Data Form}

Date

\section{CLIENT INFORMATION}

Client Name:

Age at time of participation in the study:

Date that your brain injury occurred:

Gender: $\square$ Male $\square$ Female

Phone Number:

Email Address:

Designated Caregiver Name:

Phone:

\section{SLEEP HISTORY}

How many hours do you typically sleep per night?

What time do you go to bed on a typical night?

How long does it typically take you to fall asleep?

What time do you typically wake up in the morning?

How many times do you typically wake up during the night?

Do you share your bed with other people? $\square$ Yes $\square$ No, Pets? $\square$ Yes $\square$ No

Does sharing your bed with others affect your sleep? $\square$ Yes $\square$ No If so, please explain:

Do you have a diagnosed sleep disorder or other medical diagnosis that affects your sleep?

$\square$ Yes $\square$ No If so, please list/explain:

Do you take any medication(s) to help you fall asleep? $\square$ Yes $\square$ No $\quad$ If so, please list below. 


\section{Appendix L}

\section{Caregiver Agreement Form}

Date:

Name of Study Participant:

Printed Name of Family Member or Caregiver:

Mobile Phone Number:

E-mail Address:

I am a family member or caregiver of a participant in the study entitled, Implications of a Multicomponent Sleep Program after Brain Injury: A Mixed Methods Study, at the XXX Community Re-entry Program. I agree to receive daily text messages and/or e-mails from the researcher every evening and weekend day. I understand that these messages are a reminder for me to make sure that the study participant is actively participating in the study interventions each day and is tracking daily data using the Fitbit device and the sleep diary form. I also agree to provide daily reminders to the participant to complete these steps and to make sure that the Fitbit device is charged before each nightly use.

Signature:

For additional questions, contact the researcher, Brandi Fulwider, at bf615@mynsu.nova.edu 
Appendix M

\section{Sleep is Important!}

- Sleep consumes approximately $1 / 3$ of the average person's time, making it a very important part of life. We should all be aware of how sleep affects other aspects of our daily life.

- Adequate sleep allows you to participate in other meaningful activities, including daily selfcare, work, and leisure interests. Sufficient sleep also supports good quality of life, health, and physical and mental well-being. The body also goes through many important physiological and mental health changes during sleep that support healing after injury or illness.

- Abnormal sleep often negatively affects mental and physical health and can lead to worsening effects of chronic illnesses. Impaired sleep can significantly affect driving, the ability to manage finances, and performance of job duties.

- Many people after brain injury have deficits in sleep, which can affect the recovery process and other symptoms associated with a brain injury, such as pain, cognitive deficits, fatigue, depression, and anxiety.

- There are many simple changes that you can make to help improve your sleep. By participating in this study, you will develop many healthy sleep habits that can improve your overall sleep quality and lead to improved daily functioning after getting a better night of sleep.

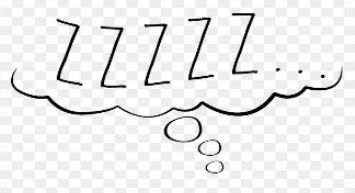




\section{Appendix N}

Fitbit Inspire HR Instruction Guide

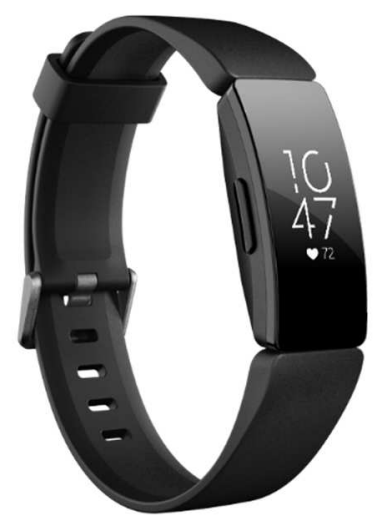

\section{Wearing the Device}

The Fitbit Inspire HR band is made of a premium silicone and fastens with an aluminum buckle and tang. Adjust the band so that it is comfortable but not too tight on the wrist. It is recommended to wear the device on the non-dominant arm if possible. You can wear the device during the day, but it must be worn every evening at bedtime and for the duration of the night while you are sleeping.

\section{Caring for the Device}

If the band gets wet or if you sweat in it, remove and completely dry the band before putting it back on. The Fitbit Inspire HR is water resistant to 50 meters, but do not wear the Fitbit in a hot tub or sauna. Give your wrist a rest by taking the band off every now and then, but do not remove it during the night when you are attempting to sleep.

\section{Battery and Power}

The Fitbit Inspire HR has up to 5 days of battery. Battery life and charge cycles vary with use, settings, and other factors. You should charge your tracker every 2-3 days to ensure that you are always tracking. Charge time $(0-100 \%)$ is 2 hours.

${ }^{* * *}$ The Fitbit Inspire HR will be synced to a device owned by the researcher 2 times per week during the study period. Do not attempt to sync the Fitbit to your personal device during the study. Bring your Fitbit with you to the community re-entry program daily so that data can be obtained from it. Also, do not change any settings on the Fitbit device during the study period.

See www.fitbit.com/productcare for more details or contact the researcher via phone (xxx-xxxxxxx) or by e-mail at bf615@mynsu.nova.edu 


\section{Appendix O}

Sample Daily Intervention Checklists

\section{Daily Intervention Checklist: (DATE)}

Intervention Week 1 (Initial Phase - Intervention B)

- Review each item every morning after waking and every night before bed.

- Follow each of the suggested items every day and night.

- Complete the checklist to designate which items were completed in the morning when filling out the Consensus Sleep Diary.

\begin{tabular}{|l|l|}
\hline $\begin{array}{l}\text { Check if } \\
\text { completed }\end{array}$ & Daily Instructions \\
\hline & 1. Lie down and go to bed only when feeling sleepy. \\
\hline & $\begin{array}{l}\text { 2. Use the bed and bedroom for sleep and sexual activity only. Do not use } \\
\text { your cell phone or watch television in bed. }\end{array}$ \\
\hline & $\begin{array}{l}\text { and go into another room to participate in calming activities. Go back to } \\
\text { bed when feeling sleepy. }\end{array}$ \\
\hline & $\begin{array}{l}\text { Set an alarm clock to get up at the same time each morning, regardless } \\
\text { of bedime. }\end{array}$ \\
\hline & Avoiding napping during the day. \\
\hline & \\
\hline & \\
\hline & \\
\hline & \\
\hline
\end{tabular}

NOTES 
Daily Intervention Checklist: (DATE)

Intervention Week 2 (Initial Phase - Intervention C)

- Review each item every morning after waking and every night before bed.

- Follow each of the suggested items every day and night.

- Complete the checklist to designate which items were completed in the morning when filling out the Consensus Sleep Diary.

\begin{tabular}{|c|c|}
\hline \multirow{2}{*}{$\begin{array}{l}\text { Check if } \\
\text { completed }\end{array}$} & Daily Instructions \\
\hline & $\begin{array}{l}\text { 1. Exercise in the late afternoon or early evening (before dinner). As an } \\
\text { alternative, take a warm bath or shower one to two hours before } \\
\text { bedtime. }\end{array}$ \\
\hline & $\begin{array}{l}\text { 2. Eat a light snack, such as crackers, bread, or fruit, before bedtime. } \\
\text { Avoid sugary snacks. }\end{array}$ \\
\hline & $\begin{array}{l}\text { 3. Avoid liquids before bedtime, drinking no more than } 6-7 \text { ounces in the } \\
\text { last four hours before bedtime. Drink a small cup of chamomile tea } \\
\text { before bedtime. }\end{array}$ \\
\hline & 4. Avoid caffeinated products within six hours of bedtime. \\
\hline & 5. Avoid alcohol and limit nicotine use, especially in the evening hours. \\
\hline & $\begin{array}{l}\text { 6. Use earplugs or some form of white noise (e.g. fan or humidifier, white } \\
\text { noise apps) to mitigate noise. }\end{array}$ \\
\hline & 7. Avoid co-sleeping with pets (if applicable). \\
\hline & 8. Make sure that bedding and pillows are clean and comfortable. \\
\hline & $\begin{array}{l}\text { 9. Keep the bedroom cool, between } 65 \text { and } 68 \text { degrees if possible or by } \\
\text { using a fan. }\end{array}$ \\
\hline & $\begin{array}{l}\text { 10. Keep the bedroom dark by covering windows with a dark blanket or } \\
\text { sheet, the use of blackout blinds or dark curtains, or by wearing an eye } \\
\text { mask. }\end{array}$ \\
\hline & \\
\hline & \\
\hline & \\
\hline & \\
\hline & \\
\hline
\end{tabular}

NOTES 
Daily Intervention Checklist: (DATE)

Intervention Week 3 (Initial Phase - Intervention D)

- Review each item every morning after waking and every night before bed.

- Follow each of the suggested items every day and night.

- Complete the checklist to designate which items were completed in the morning when filling out the Consensus Sleep Diary.

\begin{tabular}{|c|c|}
\hline Check if & Daily Instructions \\
\hline & $\begin{array}{l}\text { 1. Use earplugs or some form of white noise (e.g. fan or humidifier, white } \\
\text { noise apps) to mitigate noise. }\end{array}$ \\
\hline & 2. Avoid co-sleeping with pets (if applicable). \\
\hline & 3. Make sure that bedding and pillows are clean and comfortable. \\
\hline & $\begin{array}{l}\text { 4. Keep the bedroom cool, between } 65 \text { and } 68 \text { degrees if possible or by } \\
\text { using a fan. }\end{array}$ \\
\hline & $\begin{array}{l}\text { 5. Keep the bedroom dark by covering windows with a dark blanket or } \\
\text { sheet, the use of blackout blinds or dark curtains, or by wearing an eye } \\
\text { mask. }\end{array}$ \\
\hline & 6. Lie down and go to bed only when feeling sleepy. \\
\hline & $\begin{array}{l}\text { 7. Use the bed and bedroom for sleep and sexual activity only. Do not use } \\
\text { your cell phone or watch television in bed. }\end{array}$ \\
\hline & $\begin{array}{l}\text { 8. If unable to fall asleep or awake in bed for more than } 10 \text { minutes, get up } \\
\text { and go into another room to participate in calming activities. Go back to } \\
\text { bed when feeling sleepy. }\end{array}$ \\
\hline & $\begin{array}{l}\text { 9. Set an alarm clock to get up at the same time each morning, regardless } \\
\text { of bedtime. }\end{array}$ \\
\hline & 10. Avoiding napping during the day. \\
\hline & $\begin{array}{l}\text { 11. Exercise in the late afternoon or early evening (before dinner). As an } \\
\text { alternative, take a warm bath or shower one to two hours before } \\
\text { bedtime. }\end{array}$ \\
\hline & $\begin{array}{l}\text { 12. Eat a light snack, such as crackers, bread, or fruit, before bedtime. } \\
\text { Avoid sugary snacks. }\end{array}$ \\
\hline & $\begin{array}{l}\text { 13. Avoid liquids before bedtime, drinking no more than } 6-7 \text { ounces in the } \\
\text { last four hours before bedtime. Drink a small cup of chamomile tea } \\
\text { before bedtime. }\end{array}$ \\
\hline & 14. Avoid caffeinated products within six hours of bedtime. \\
\hline & 15. Avoid alcohol and limit nicotine use, especially in the evening hours. \\
\hline
\end{tabular}

NOTES 


\section{Appendix P \\ Intervention Phases Educational Handouts \\ Instructions for Intervention B Phase}

Follow each of the instructions listed below from today until the end of the study period.

1. Lie down and go to bed only when feeling sleepy.

2. Use the bed and bedroom for sleep and sexual activity only. Do not use your cell phone or watch television in bed.

3. If unable to fall asleep or awake in bed for more than 10 minutes, get up and go into another room to participate in calming activities. Go back to bed when feeling sleepy.

4. Set an alarm clock to get up at the same time each morning, regardless of bedtime.

5. Avoiding napping during the day.

* These instructions lead to learning to associate the bed and the bedroom with sleep and help to reduce cues that associate the bed and bedroom with arousal. By lying down and going to bed only when feeling sleepy, it makes a person more aware of the body's cues related to sleepiness.

Using the bed for sleep only allows individuals to associate the bed and bedroom with falling asleep, and leaving the bedroom when unable to fall asleep limits the amount of time of being awake while in bed. Do not participate in other activities in bed, and do not worry while in bed. Setting an alarm clock to get up at the same time each morning helps to set a consistent sleep rhythm. Avoiding napping during the day allows any sleep deprivation from the previous night to help facilitate sleep the following night.

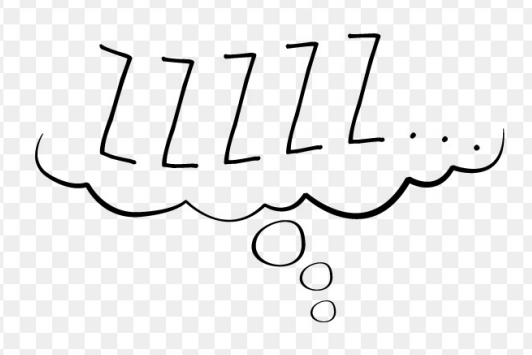

(Bootzin \& Perlis, 2011) 


\section{Instructions for Intervention C Phase}

Follow each of the instructions listed below from today until the end of the study period.

1. Exercise in the late afternoon or early evening (before dinner). As an alternative, take a warm bath or shower one to two hours before bedtime.

2. Eat a light snack, such as crackers, bread, or fruit, before bedtime. Avoid sugary snacks.

3. Avoid too many liquids before bedtime, drinking no more than 6-7 ounces in the last four hours before bedtime. Drink a small cup of chamomile tea before bedtime.

4. Avoid caffeinated products within six hours of bedtime.

5. Avoid alcohol and limit nicotine use, especially in the evening hours.

* These interventions encourage behavioral changes that can support improved sleep. Exercise can help to deepen sleep and provide more restorative sleep, but should be done in the late afternoon or early evening so that it is not arousing. It is best to avoid going to bed on a full stomach or while feeling hungry, and a light snack that includes carbohydrates may be beneficial about one hour before bedtime. Liquids should be restricted in the last four hours before bedtime to limit having to use the restroom in the middle of the night. Caffeine and alcohol should also be avoided in evening hours, as they can significantly disturb sleep. While alcohol can decrease the time required to fall asleep, it often results in fragmented sleep later in the night. The general rule for consumption of caffeine is that it should be avoided at least six hours before sleep, and nicotine should be avoided close to bedtime as well because it can be a stimulant.

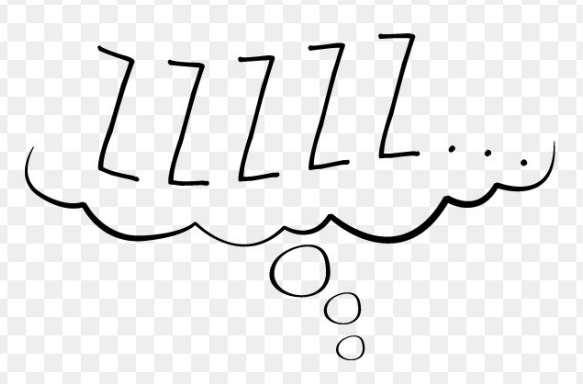

(Green \& Hicks, 2015; Posner \& Gehrman, 2011) 


\section{Instructions for Intervention D Phase}

Follow each of the instructions listed below from today until the end of the study period.

1. Use earplugs or some form of white noise (e.g. fan or humidifier, white noise apps) to mitigate noise.

2. Avoid co-sleeping with pets (if applicable).

3. Make sure that bedding and pillows are clean and comfortable.

4. Keep the bedroom cool, between 65 and 68 degrees if possible or by using a fan.

5. Keep the bedroom dark by covering windows with a dark blanket or sheet, the use of blackout blinds or dark curtains, or by wearing an eye mask.

* These interventions encourage environmental changes that can support improved sleep. As a general rule, the bedroom should always be conducive to sleep. Bedrooms should be kept cool, should have good ventilation, and should be quiet and dark. Earplugs or the use of white noise can help to drown out other disruptive noises. Avoiding co-sleeping with one's pets can reduce environmental disruptions and distractions that might inhibit good sleep.

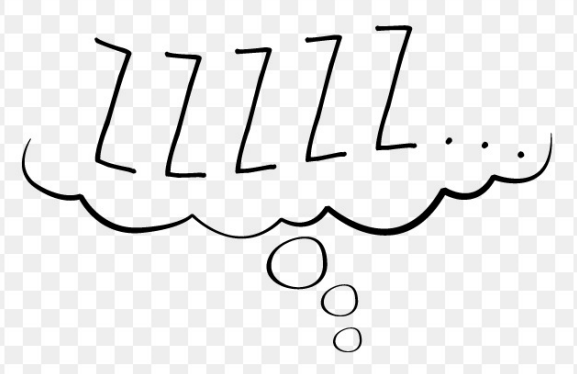

(Posner \& Gehrman, 2011) 
Appendix Q

Copyright Permissions

Consensus Sleep Diary

Colleen Carney

Apr 15, 2019

To You

SH paper_Behaviour Therapist

PDF - 3.3 MB

NSU Security WARNING: This is an external email. Do not click links or open attachments unless you recognize the sender and know that the content is safe.

Hi Brandi.

You can have permission for this listed study as long as you cite the original paper.

I am not sure if you have started this study or not, or if the hypothesis is that it will NOT be effective, but I have attached a paper (it starts on the third paper) about how Sleep Hygiene is not effective (e.g., Morin et al., 1999; 2006). You should know that it is frequently used as a behavioural placebo in clinical trials (e.g., Carney et al., 2017) and that the American Academy of Sleep Medicine is about to advise against it's use in its upcoming Clinical Practice Parameters.

Best

Colleen 
Epworth Sleepiness Scale

\section{ePROVIDE' ${ }^{\text {TM: }}$ Your User Agreement - Epworth Sleepiness Scale - 2345}

E eprovide@mapi-trust.org

UA_General_Ter... PDF - 559 KB

\section{UA_special_term...} 1. PDF $-28 \mathrm{~KB}$

\section{2 attachments (587 KB)}

Dear User,

Thank you for using the online distribution on https://eprovide.mapi-trust.org. for the use of this COA.

Please find attached your completed User License Agreement. 
Functional Outcomes of Sleep Questionnaire

\section{Re: Request for permission to use the FOSQ}

$\mathrm{RA}$

Ruiz, Alice

To You
Apr 16, 2019

Fosq98 shol

PDF - $80 \mathrm{~KB}$

\section{O attachments $(517 \mathrm{~KB})$}

Hello:

Attached please find the FOSQ 30 and FOSQ 10 as well as the letter of agreement and list of translations. Once I receive the signed letter of agreement, the scoring instructions will be sent to you. Please let me know if you have any questions.

Thank you,

Alice 
Insomnia Severity Index

ePROVIDE ${ }^{\mathrm{TM}}$ : Your User Agreement - Insomnia Severity Index - 2344

E eprovide@mapi-trust.org Apr 19, 2019 To You

UA_General_Ter...

므. PDF $-559 \mathrm{~KB}$

UA_special_term...

10. PDF $-28 \mathrm{~KB}$

2 attachments (587 KB)

Dear User,

Thank you for using the online distribution on https://eprovide.mapi-trust.org. for the use of this COA.

Please find attached your completed User License Agreement. 
Sleep Hygiene Index

\section{DM}

David Mastin

Apr 16, 2019

To You

AU Formatted SHI (1) (2) (1) ...
PDF - $128 \mathrm{~KB}$

言畐

SHI (2) (1) (1

PDF - $168 \mathrm{~KB}$

3 attachments (360 KB)

NSU Security WARNING: This is an external email. Do not click links or open attachments unless you recognize the sender and know that the content is safe.

Hi Brandi,

There are no special permissions required other than providing a citation to the original publication in the journal of behavioral medicine.

Let me know if I can help.

Sincerely,

David 